\title{
Limited Feedback Techniques in Multiple Antenna Wireless Communication Systems
}

by

Jawad Mirza

\author{
A thesis \\ submitted to the Victoria University of Wellington \\ in fulfilment of the \\ requirements for the degree of \\ Doctor of Philosophy \\ in Engineering.
}

Victoria University of Wellington 2015 


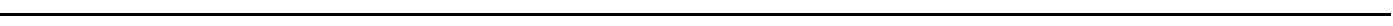




\section{Acknowledgments}

Firstly, I would like to thank Almighty "Allah" for giving me strength and wisdom throughout my life.

I would like to express my sincere appreciation to my supervisors Dr. Pawel Dmochowski and Prof. Mansoor Shafi. I am very grateful to Pawel for his continuous support and encouragement throughout my research. He provided me with opportunities and dedicated invaluable time to this research work. I also have been truly fortunate to be one of the students of Prof. Mansoor Shafi, who always guided my research towards the right direction. More than anything else, he has taught me how to be a thoughtful person.

I am also grateful to Prof. Peter Smith for providing valuable technical and non-technical suggestions to improve my research work. He has always been there to answer my innumerable questions related to the research and his willingness to solve the problems analytically is very inspiring. I would also like to thank Dr. Abdulla Firag and Dr. Min Zhang for helping me to understand some critical concepts at the start of my $\mathrm{PhD}$. Sincere appreciation also goes to Dr. Paul Teal, Dr. Alan J. Coulson and Dr. Vasanthan Raghavan for examining the thesis and providing useful suggestions.

I am very grateful to have had the chance to share this journey with extraordinary friends and amazing colleagues. I am thankful to their presence, support and guidance. I want to thank Muhammad Adeel Mahmood, Saqib Saleem, Harsh Tataria, Ramoni O. Adeogun, Saud Naqvi, Afraz Liaquat, Tahir Suleman, Asim Masood, Abdul Wahid, Muhammad Ibrahim, Mudassir Altaf, Muhammad Iqbal, Refik Fatih Ustok, Callum Neil and Ahmed Sheik Deeb.

Finally, I would like to express my deepest gratitude to my family for their unconditional love, support and sacrifice. I would like to thank my parents for teaching me the value of education, patience and perseverance. I would like to thank my sisters for showering me with affection and for encouraging me. I am greatly indebted to my brother and best friend Ammar. I am sincerely grateful to my wife for her cooperation and support. Last but not least, I dedicate this work to my beautiful daughter Ayra, whom I love and adore. 


\section{Abstract}

Multiple antenna systems provide spatial multiplexing and diversity benefits. These systems also offer beamforming and interference mitigation capabilities in single-user (SU) and multi-user (MU) scenarios, respectively. Although diversity can be achieved without channel state information (CSI) at the transmitter using space-time codes, the knowledge of instantaneous CSI at the transmitter is essential to the above mentioned gains. In frequency division duplexing (FDD) systems, limited feedback techniques are employed to obtain CSI at the transmitter from the receiver using a low-rate link. As a consequence, CSI acquired by the transmitter in such manner have errors due to channel estimation and codebook quantization at the receiver, resulting in performance degradation of multi-antenna systems. In this thesis, we examine CSI inaccuracies due to codebook quantization errors and investigate several other aspects of limited feedback in SU, MU and multicell wireless communication systems with various channel models.

For SU multiple-input multiple-output (MIMO) systems, we examine the capacity loss using standard codebooks. In particular, we consider single-stream and two-stream MIMO transmissions and derive capacity loss expressions in terms of minimum squared chordal distance for various MIMO receivers. Through simulations, we investigate the impact of codebook quantization errors on the capacity performance in uncorrelated Rayleigh, spatially correlated Rayleigh and standardized MIMO channels. This work motivates the need of effective codebook design to reduce the codebook quantization errors in correlated channels.

Subsequently, we explore the improvements in the design of codebooks in temporally and spatially correlated channels for MU multiple-input single-output (MISO) systems, by employing scaling and rotation techniques. These codebooks quantize instantaneous channel direction information (CDI) and are referred as differential codebooks in the thesis. We also propose various adaptive scaling techniques for differential codebooks where packing density of codewords 
in the differential codebook are altered according to the channel condition, in order to reduce the quantization errors. The proposed differential codebooks improve the spectral efficiency of the system by minimizing the codebook quantization errors in spatially and temporally correlated channels.

Later, we broaden the scope to massive MISO systems and propose trellis coded quantization (TCQ) schemes to quantize CDI. Unlike conventional codebook approach, the TCQ scheme does not require exhaustive search to select an appropriate codeword, thus reducing computational complexity and memory requirement at the receiver. The proposed TCQ schemes yield significant performance improvements compared to the existing TCQ based limited feedback schemes in both temporally and spatially correlated channels.

Finally, we investigate interference coordination for multicell MU MISO systems using regularized zero-forcing (RZF) precoding. We consider random vector quantization (RVQ) codebooks and uncorrelated Rayleigh channels. We derive expected SINR approximations for perfect CDI and RVQ codebook-based CDI. We also propose an adaptive bit allocation scheme which aims to minimize the network interference and moreover, improves the spectral efficiency compared to equal bit allocation and coordinated zero-forcing $(\mathrm{ZF})$ based adaptive bit allocation schemes. 


\section{Acronyms}

\begin{tabular}{|c|c|}
\hline $3 \mathrm{GPP}$ & 3rd Generation Partnership Project \\
\hline AoA & Angle of Arrival \\
\hline AoD & Angle of Departure \\
\hline BER & Bit error rate \\
\hline $\mathrm{BS}$ & Base Station \\
\hline $\mathrm{CDF}$ & Cumulative Distribution Function \\
\hline CDI & Channel Direction Information \\
\hline $\mathrm{CF}$ & Characteristic Function \\
\hline CIR & Channel Impulse Response \\
\hline CSI & Channel State Information \\
\hline DoF & Degree of Freedom \\
\hline $\mathrm{EC}$ & Ergodic Capacity \\
\hline ECL & Ergodic Capacity Loss \\
\hline FDD & Frequency Division Duplexing \\
\hline FOGM & First-Order Gauss-Markov \\
\hline GLP & Grassmannian Line Packing \\
\hline
\end{tabular}




$\begin{array}{ll}\text { i.i.d. } & \text { Independent and Identically Distributed } \\ \text { ICI } & \text { Inter-Cell Interference } \\ \text { IMT } & \text { International Mobile Telecommunications } \\ \text { ITU } & \text { International Telecommunication Union } \\ \text { IUI } & \text { Inter-User Interference } \\ \text { JP } & \text { Joint Processing } \\ \text { KKT } & \text { Karush-Kuhn-Tucker } \\ \text { LoS } & \text { Line-of-Sight } \\ \text { LTE } & \text { Long Term Evolution } \\ \text { MF } & \text { Matched-Filter } \\ \text { MIMO } & \text { Multiple-Input-Multiple-Output } \\ \text { MISO } & \text { Multiple-Input-Single-Output } \\ \text { MMSE } & \text { Minimum-Mean Squared Error } \\ \text { mmWave } & \text { Millimeter Wave } \\ \text { MU } & \text { Multi-user }\end{array}$

HetNet Heterogeneous Network

NLoS Non-Line-of-Sight

NTCQ Non Coherent Trellis Coded Quantization

O2I Outdoor-to-Indoor

OFDM Orthogonal Frequency Division Multiplexing 


\begin{tabular}{|c|c|}
\hline PDF & Probability Density Function \\
\hline RMa & Rural Macro \\
\hline RVQ & Random Vector Quantization \\
\hline RZF & Regularized Zero-Forcing \\
\hline $\mathrm{SCM}$ & Spatial Channel Model \\
\hline SCME & Spatial Channel Model Extended \\
\hline SIMO & Single-Input-Multiple-Output \\
\hline SINR & Signal-to-Interference-Plus-Noise Ratio \\
\hline SISO & Single-Input-Single-Output \\
\hline SLNR & Signal-to-Leakage-Plus-Noise Ratio \\
\hline SMa & Sub-urban Macro \\
\hline SNR & Signal-to-Noise Ratio \\
\hline $\mathrm{SU}$ & Single-user \\
\hline SVD & Singular Value Decomposition \\
\hline TCQ & Trellis Coded Quantization \\
\hline TDD & Time Division Duplexing \\
\hline ULA & Uniform linear array \\
\hline UMa & Urban Macro \\
\hline UMi & Urban Micro \\
\hline URA & Uniform rectangular array \\
\hline
\end{tabular}


WINNER Wireless World Initiative New Radio

$\mathrm{ZF}$

Zero-Forcing 


\section{Notation and Symbols}

\begin{tabular}{|c|c|}
\hline $\mathcal{N}\left(\mu, \sigma^{2}\right)$ & Normal distribution with mean $\mu$ and variance $\sigma^{2}$ \\
\hline $\mathcal{C N}\left(\mu, \sigma^{2}\right)$ & Complex normal distribution with mean $\mu$ and variance $\sigma^{2}$ \\
\hline $\mathbb{R}^{+}$ & Set of positive real numbers \\
\hline $\mathbb{Z}^{+}$ & Set of positive integers \\
\hline$|\cdot|$ & Absolute value \\
\hline$\|\cdot\|$ & Euclidean norm of a vector \\
\hline$\|\cdot\|_{F}$ & Frobenius norm of a matrix \\
\hline $\mathbb{E}[\cdot]$ & Expected value \\
\hline $\operatorname{det}(\cdot)$ & Determinant of a matrix \\
\hline $\operatorname{tr}(\cdot)$ & Trace of a matrix \\
\hline $\log _{2}(\cdot)$ & Logarithm to the base 2 \\
\hline $\log _{10}(\cdot)$ & Logarithm to the base 10 \\
\hline $\ln (\cdot)$ & Natural logarithm \\
\hline$e^{(\cdot)}$ & Exponential function \\
\hline$\beta(\cdot, \cdot)$ & Beta function \\
\hline$J_{0}(\cdot)$ & Bessel function of zeroth order \\
\hline
\end{tabular}




$$
L(\cdot, \cdot) \quad \text { Lagrange function }
$$

$\operatorname{SVD}(\cdot) \quad$ Singular value decomposition

$\operatorname{Ei}(\cdot, \cdot) \quad$ Generalized exponential integral

$\Gamma(\cdot, \cdot) \quad$ Incomplete gamma function

$(\cdot)^{T} \quad$ Matrix or vector transpose

$(\cdot)^{\perp} \quad$ Null space of a vector

$(\cdot)^{-1} \quad$ Inverse of a Matrix

$(\cdot)^{H} \quad$ Conjugate transpose of a matrix and a vector

$(\cdot)^{+} \quad \max (\cdot, 0)$

$\Re(\cdot) \quad$ Real part of a complex number

$\Im(\cdot) \quad$ Imaginary part of a complex number

$\lfloor x\rfloor \quad$ Largest integer less than or equal to $x$

$f(x) \quad$ probability density function of $x$

$F(x) \quad$ Cumulative distribution function of $x$

I Identity matrix

$P_{t} \quad$ Transmit power

$P_{r} \quad$ Receive power

$P_{0} \quad$ Effective radiated power

$R_{c} \quad$ Cell radius

$a$

Path loss exponent 
$\Phi$

$\sigma_{S F}$

$t$

$\tau$

$T_{c}$

$D_{s}$

$f_{c}$

$T_{\text {req }}$

$B_{c}$

$T_{d}$

$B_{s}$

$n_{t}$

$n_{r}$

$\mathbf{H}$

$\mathbf{H}_{\text {Rician }}$

h

$\hat{\mathbf{H}}$

$\hat{\mathbf{h}}$

$\hat{\mathbf{h}}_{f}$

$\tilde{\mathbf{H}}$
Lognormal Shadow fading

Shadow fading standard deviation

Time

Delay

Coherence time of the channel

Doppler spread

Carrier frequency

Application delay requirement

Coherence bandwidth of the channel

Delay spread

Bandwidth of the signal

Number of transmit antennas

Number of receive antennas

i.i.d. Rayleigh fading MIMO channel

Rician MIMO channel

i.i.d. Rayleigh fading MISO channel

Spatially correlated MIMO channel

Spatially correlated MISO channel

Full Spatially correlated MISO channel

Temporally correlated MIMO channel 
$\tilde{\mathbf{h}}$

Temporally correlated MISO channel

$\overline{\mathrm{h}}$

Spatially/temporally correlated MISO channel

$\ddot{\mathrm{h}}$

Estimated MISO channel

$\mathbf{u}$

Channel direction information

q

Quantized channel direction information

g

Quantized channel state information

$\mathcal{F}_{\mathrm{S}-\mathrm{S}}$

Codebook for single-stream MIMO or MISO transmission

$\mathcal{F}_{\mathrm{M}-\mathrm{S}}$

Codebook for multi-stream MIMO transmission

$K_{f} \quad$ Rician K-factor

$\mathbf{R}_{t} \quad$ Transmit correlation matrix

$\mathbf{R}_{r} \quad$ Receive correlation matrix

$z_{t}$

Transmit correlation coefficient

$z_{r}$

Receive correlation coefficient

$\epsilon$

Temporal correlation coefficient

$f_{d} \quad$ Maximum Doppler frequency

$T_{s} \quad$ Channel sample time

$\phi \quad$ Azimuth angle-of-arrival

$\varphi \quad$ Azimuth angle-of-departure

$f_{c} \quad$ Carrier frequency

$\lambda_{0} \quad$ Wavelength of a carrier frequency 


\begin{tabular}{|c|c|}
\hline$F_{i, V}$ & Receive vertical antenna field pattern \\
\hline$F_{i, H}$ & Receive horizontal antenna field pattern \\
\hline$F_{k, V}$ & Transmit vertical antenna field pattern \\
\hline$F_{k, H}$ & Transmit horizontal antenna field pattern \\
\hline$v$ & Velocity of the receiver \\
\hline$\varpi$ & Cross polarization power ratio (XPR) \\
\hline$M_{r}$ & Number of rays in the cluster \\
\hline$N_{s}$ & Number of scatterers/cluster \\
\hline$P_{n}$ & Power of the $n^{\text {th }}$ cluster \\
\hline$\Theta_{n, m}^{x y}$ & $\begin{array}{l}\text { Random phase offset of the } n^{\text {th }} \text { cluster and the } m^{\text {th }} \text { ray between } \\
\text { the x component of the transmitter element and the y component } \\
\text { of the reciver element. }\end{array}$ \\
\hline$\rho$ & Signal-to-noise ratio (SNR) \\
\hline $\bar{C}$ & MIMO Capacity with full CSI at transmitter \\
\hline$C_{\mathrm{M}}$ & MIMO Capacity with no CSI at transmitter \\
\hline$\tilde{C}_{\mathrm{M}}$ & MIMO Capacity with waterfilling \\
\hline$C_{\mathrm{S}}$ & SISO Capacity \\
\hline f & MIMO precoding vector for single-stream transmission \\
\hline $\mathbf{F}$ & MIMO precoding matrix for multi-stream transmission \\
\hline$D_{f}$ & Distortion function for MIMO/MISO \\
\hline $\mathbf{U}$ & Left unitary matrix of the channel \\
\hline
\end{tabular}




\begin{tabular}{|c|c|}
\hline $\mathbf{u}_{\mathrm{dom}}$ & Dominant left unitary eigenvector of the channel \\
\hline $\mathbf{V}$ & Right unitary matrix of the channel \\
\hline $\mathbf{v}_{\text {dom }}$ & Dominant right unitary eigenvector of the channel \\
\hline$C_{\text {MIMO-SM }}$ & Capacity of MIMO spatial multiplexing system \\
\hline$C_{\text {sum }}$ & Spectral efficiency of the MU system \\
\hline$L$ & Number of transmission streams \\
\hline$K$ & Number of users in the MU MISO system \\
\hline SINR & SINR at the receiver \\
\hline $\mathbf{W}_{\mathrm{zf}}$ & ZF Precoding matrix \\
\hline $\mathbf{W}_{\mathrm{rzf}}$ & RZF Precoding matrix \\
\hline$\zeta$ & RZF regularization parameter \\
\hline $\mathbf{w}$ & Precoding vector for a MU MISO system \\
\hline$B$ & Number of feedback bits \\
\hline$N_{c}$ & Number of entries in the codebook \\
\hline $\mathcal{Q}$ & Codebook quantization error \\
\hline$R$ & Capacity Ratio \\
\hline$D$ & Capacity difference \\
\hline$\tilde{R}$ & Capacity ratio with full CSI at transmitter \\
\hline$\tilde{D}$ & Capacity difference with full CSI at transmitter \\
\hline
\end{tabular}


$d_{\mathrm{dmin}} \quad$ Minimum squared chordal distance

$\Omega_{\mathrm{SVD}} \quad$ SVD receive filter

$\Omega_{\mathrm{ZF}} \quad$ ZF receive filter

$\boldsymbol{\Omega}_{\mathrm{MMSE}} \quad$ MMSE receive filter

$N_{\text {sub }} \quad$ Number of subcarriers in OFDM systems

$C_{r} \quad$ Number of channel realizations

$s_{k} \quad$ Data symbol for the $k^{\text {th }}$ user

$n_{k} \quad$ Noise term for the $k^{\text {th }}$ user

$\sigma_{k}^{2} \quad$ Noise variance for the $k^{\text {th }}$ user

$\Omega_{n_{t}} \quad$ One-dimensional complex Stiefel manifold on $\mathbb{C}^{n_{t}}$

$G\left(n_{t}, 1\right) \quad$ Complex Grassmannian manifold of $n_{t}$ dimension

$\mathbb{O}(\mathbf{c}, \varsigma) \quad$ Spherical-cap with center $\mathbf{c}$ and radius $\varsigma$

$\alpha \quad$ Scaling parameter for the spherical-cap

$\Theta \quad$ Rotation matrix

$\tilde{\alpha} \quad$ Adaptive Scaling Technique I for spherical-cap

$\tilde{\alpha}^{*} \quad$ Adaptive Scaling Technique II for spherical-cap

$\mathrm{d}_{\text {mean }} \quad$ Mean channel variation in terms of chordal distance

$\mathrm{d}_{\text {error }} \quad$ Mean quantization error in terms of chordal distance

$\mathbf{F}_{\text {spherical }} \quad$ Spherical-cap codebook

$\mathbf{F}_{\text {polar }} \quad$ Polar-cap codebook 
$\alpha_{p} \quad$ Radius of Polar-cap in double-cap differential codebook

$\alpha_{s} \quad$ Radius of Spherical-cap in double-cap differential codebook

$\chi$

Estimation error coefficient

$d_{\text {Euclidean }} \quad$ Mean Euclidean distance between channel entries over time

$\kappa_{c} \quad$ Scaling parameter for the source constellation

$q$

Ratio between number of transmit antenna and number of users

$N \quad$ Number of states in a trellis

$L_{c} \quad$ Number of constellation points

C Number of coordinating cells

$\gamma_{c} \quad$ Normalization parameter for RZF precoding matrix in the $c^{\text {th }}$ cell

$\mathbf{E}_{c} \quad$ Concatenated quantization error matrix

$\mathbf{T}_{c} \quad$ Concatenated quantized channel matrix

$\delta_{k, c} \quad$ Expected signal power (without pathloss and shadowing) at the $k^{\text {th }}$ user in the $c^{\text {th }}$ cell

$\bar{\gamma}_{c} \quad$ Expected value of normalization parameter for RZF precoding matrix in the $c^{\text {th }}$ cell

$\psi_{c} \quad$ Expected interference power (without pathloss and shadowing) at the user from the $c^{\text {th }}$ cell

$\psi_{j} \quad$ Expected interference power (without pathloss and shadowing) at the user from the $j^{\text {th }}$ cell 


\section{Contents}

$\begin{array}{ll}\text { Acknowledgments } & \text { i }\end{array}$

Acronyms $\quad$ v

$\begin{array}{ll}\text { Notation and Symbols } & \text { ix }\end{array}$

1 Introduction $\quad 1$

1.1 Multi-Antenna Downlink Systems . . . . . . . . . . . . . 1

1.1.1 Single User Systems . . . . . . . . . . . . . . . . . . . . . . . . . . . . . . .

1.1 .2 Multiuser Systems . . . . . . . . . . . . . . 2

1.1.3 Multicell Systems . . . . . . . . . . . . . . . . 2

1.1.4 Massive Multi-antenna Systems . . . . . . . . . . . 3

1.2 Limited Feedback Techniques . . . . . . . . . . . . . . . 3

1.3 Research Framework . . . . . . . . . . . . . . . . 5 5

1.3.1 Motivation . . . . . . . . . . . . . . 5

1.3 .2 Contributions . . . . . . . . . . . . . 6

1.3.3 List of Publications . . . . . . . . . . . . . 8

1.4 Thesis Outline . . . . . . . . . . . . . . . . . . . . . 9

2 Wireless Channels $\quad 11$

2.1 Wireless Radio Channel . . . . . . . . . . . . . . . . . 11

2.2 Analytical MIMO Channel Models . . . . . . . . . . . . . . . . 14

2.2.1 Rayleigh i.i.d. Channel . . . . . . . . . . . . . . 14

2.2.2 Rician Channel . . . . . . . . . . . . . . . . . . . . . . . 14

2.2.3 Spatially Correlated Rayleigh Channel . . . . . . . . . 15

2.2.4 Temporally Correlated Rayleigh Channel . . . . . . . . . 15

2.3 Standardized MIMO Channel Models . . . . . . . . . . . . . . 16

2.3.1 3GPP Spatial Channel Model . . . . . . . . . . . . . 16

2.3.2 WINNER Channel Models . . . . . . . . . . . . . 17

2.4 Capacity of MIMO Wireless Channels . . . . . . . . . . . . . . . 21

2.4.1 Perfect CSI at Transmitter . . . . . . . . . . . . . . . 21

2.4 .2 No CSI at Transmitter . . . . . . . . . . . . . . 22

2.5 Summary . . . . . . . . . . . . . . . . . . . . . . . 22 
3 Theoretical Background of Limited Feedback Systems 23

3.1 Limited Feedback Multiple Antenna Systems . . . . . . . . . . . 23

3.2 SU Systems with Limited Feedback . . . . . . . . . . . . . . . . 24

3.2.1 Beamforming . . . . . . . . . . . . . . 24

3.2 .2 Spatial Multiplexing . . . . . . . . . . . . 26

3.3 MU Systems with Limited Feedback . . . . . . . . . . . . . . . 26

3.3.1 ZF Precoding . . . . . . . . . . . . . . . 27

3.3.2 RZF Precoding . . . . . . . . . . . . . . . . 27

3.3.3 MF Precoding . . . . . . . . . . . . . . . . . . . . . 28

3.3.4 SLNR Precoding . . . . . . . . . . . . . . . . . . . 28

3.4 Codeword Selection . . . . . . . . . . . . . . . . . . . . . . 29

3.4.1 SNR Maximization . . . . . . . . . . . . . 29

3.4 .2 Squared Chordal Distance . . . . . . . . . . . . . . 30

3.4.3 Capacity Maximization . . . . . . . . . . . . . . . . 30

3.4.4 Random Selection . . . . . . . . . . . . . . . . . . 31

3.5 Codebook Quantization Errors . . . . . . . . . . . . . . . 31

3.6 Feedback Update Interval . . . . . . . . . . . . . . . . . . . 32

3.7 MIMO Channels and Codebooks . . . . . . . . . . . . . 32

3.8 Summary . . . . . . . . . . . . . . . . . . . . . 34

4 MIMO Capacity Analysis $\quad 35$

4.1 MIMO Capacity Gains . . . . . . . . . . . . . . . . . . . . . 35

4.2 MIMO Capacity Loss Analysis with Limited Feedback . . . . . 38

4.2 .1 System Description . . . . . . . . . . . . . . . . . . 39

4.2.2 Capacity Loss in ZF and MMSE Recivers . . . . . . . . . 41

4.2.3 Capacity Loss in SVD Reciever . . . . . . . . . . . . 43

4.2.4 Numerical Results and Discussion . . . . . . . . . . . . 44

4.3 Summary . . . . . . . . . . . . . . . . . 50

5 MU MISO Systems with Differential Codebooks 51

5.1 Motivation and Related Work . . . . . . . . . . . . . . . . 51

5.2 Downlink System Description . . . . . . . . . . . . . . 53

5.3 Spherical-Cap Differential Codebook . . . . . . . . . . . 54

5.4 Feedback Procedure . . . . . . . . . . . . . . . . . 55

5.5 Tracking Channel with Spherical-Cap . . . . . . . . . . . . 56

5.6 Rotation Operation on Codebooks . . . . . . . . . . . . . . 57

5.7 Scaling Operation on Codebooks . . . . . . . . . . . . 57

5.7.1 Adaptive Scaling Technique I . . . . . . . . . . . . 57

5.7 .2 Adaptive Scaling Technique II . . . . . . . . . . . . . 58

5.8 Double-Cap Differential Codebook . . . . . . . . . . . . . . 61

5.8 .1 Double-Cap Design . . . . . . . . . . . . . 62

5.8 .2 Blind Adaptive Scaling . . . . . . . . . . . . . . . . 64 
5.9 Numerical Results and Discussion . . . . . . . . . . . . . . 65

5.9.1 Comparison of Differential Codebooks . . . . . . . . 65

5.9.2 Differential Codebooks Versus User Velocity . . . . . . . 66

5.9 .3 Estimation Errors . . . . . . . . . . . . . . 67

5.9.4 Performance of Spherical-Cap Differential Codebooks . . 68

5.9.5 Performance of Double-Cap Differential Codebook . . . . 73

5.10 Summary . . . . . . . . . . . . . . . 75

6 MU Massive MISO Systems with TCQ $\quad 77$

6.1 Motivation and Related Work . . . . . . . . . . . . . . . 77

6.2 System Description . . . . . . . . . . . . . . . . . . . 79

6.3 Expected SINR with MF Precoding . . . . . . . . . . 79

6.3 .1 Perfect CDI . . . . . . . . . . . . . 80

6.3 .2 RVQ Codebook CDI . . . . . . . . . . . . 81

6.4 Impracticality of RVQ Codebooks For Massive MISO . . . . . . 82

6.5 Limited Feedback with TCQ . . . . . . . . . . . . 83

6.6 Differential TCQ Scheme for Temporally Correlated Channels . 87

6.6.1 Transformed Source Constellation . . . . . . . . . . . 87

6.6.2 2D Translation and Scaling Techniques . . . . . . . . 89

6.7 TCQ Scheme for Spatially Correlated Channels . . . . . . . . . 91

6.8 Numerical Results and Discussion . . . . . . . . . . . . . . . . . 94

6.8.1 Temporally Correlated Channels . . . . . . . . . . . 95

6.8.2 Spatially Correlated Channels . . . . . . . . . . . . . 99

6.8.3 WINNER II Channels . . . . . . . . . . . . . . . . . . 101

6.9 Summary ........................ 102

7 Coordinated Multicell MU MISO Systems 103

7.1 Motivation and Related Work . . . . . . . . . . . . . . . . 103

7.2 Downlink System Description . . . . . . . . . . . . . . 105

7.3 Coordinated RZF Precoding . . . . . . . . . . . . 106

7.3.1 Expected SINR with Perfect CDI . . . . . . . . . . 107

7.3.2 Expected SINR with RVQ CDI . . . . . . . . . . 111

7.4 Adaptive Bit Allocation . . . . . . . . . . . . . . . . . 115

7.5 Regularization Parameter . . . . . . . . . . . . . 117

7.6 Numerical Results and Discussion . . . . . . . . . . . . . . . . 118

7.6.1 Coordination Area . . . . . . . . . . . . . . . . . . . 118

7.6.2 SINR and Spectral Efficiency . . . . . . . . . . . 119

7.6.3 Performance of Adaptive Bit Allocation Scheme . . . . . 122

7.7 Summary . . . . . . . . . . . . . . . . . . . . . . 125 
8 Conclusions and Future Work $\quad 127$

8.1 Summary . . . . . . . . . . . . . . . . . . . . 127

8.2 Conclusions . . . . . . . . . . . . . . . . . . . . . . . . . . . 129

8.3 Limitations and Future Work . . . . . . . . . . . . . . 130

8.3.1 Limitations . . . . . . . . . . . . . . . . . . 130

8.3 .2 Future Work . . . . . . . . . . . . . . . . . 131

$\begin{array}{ll}\text { Appendices } & 135\end{array}$

$\begin{array}{ll}\text { A Proof of Result } 1 & 137\end{array}$

B Proof of Result 2 141 


\section{1 \\ Introduction}

In this chapter, a brief overview of limited feedback in multiple antenna communication systems is presented, followed by the research motivation and major thesis contributions. The outline of the thesis is provided in the last section of this chapter.

\subsection{Multi-Antenna Downlink Systems}

The use of multiple antennas at the transmitter and the receiver have enabled wireless communication systems to improve capacity, quality and reliability [1]. Such systems are known as multiple-input multiple-output (MIMO) systems. The research on MIMO was pioneered by Winters [2], Teletar [3], Alamouti [4] and Foschini and Gans [5]. In MIMO systems, spatial multiplexing and spatial diversity techniques are used to improve the capacity and the reliability, respectively. Spatial multiplexing divides the data into multiple streams and transmits each stream on a different antenna, hence increasing the capacity of the system, the increase being referred to as multiplexing gain [6,7]. On the other hand, spatial diversity techniques achieve diversity and array gain at the transmitter (transmit diversity) and at the receiver (receive diversity) in turn improving the reliability of the system [1,8-10]. The trade-off between spatial multiplexing and spatial diversity have been extensively studied in [11-15] (and references within). In addition to the MIMO antenna configuration, there are two other types of antenna configurations: single-input multiple-output (SIMO) and multiple-input single-output (MISO). SIMO has a single transmit antenna and multiple receive antennas, whereas MISO has multiple transmit antennas and a single receive antenna. 


\subsubsection{Single User Systems}

In single user (SU) systems, SIMO channels can achieve receive diversity using combining techniques at the receiver, but transmit diversity is not applicable to SIMO channels. Conversely, receive diversity is not applicable to MISO channels but such channels can achieve transmit diversity with or without channel knowledge at the transmitter [10]. Transmit beamforming techniques [8,16-19] with channel state information (CSI) at the transmitter, have been shown to improve the performance of the system in fading channels, by using complex weights at the transmit antennas [20]. The transmit beamforming increases the link signal-to-noise ratio (SNR) and also achieves higher array gains with perfect CSI. However, these gains are somewhat reduced if imperfect CSI is available at the transmitter $[17,21,22]$.

\subsubsection{Multiuser Systems}

Multiple antennas can be used at the transmitter to support multiple users in the same time/frequency resource by assigning one or more antennas per user. Such systems are called multiuser (MU) communication systems. Modern cellular communication systems are multiuser systems, where on downlink the transmitter or base station (BS) uses multiple antennas to serve multiple users. Due to multiple users, MU systems achieve MU diversity [23-25] that provides improvement in the performance as the system resources are only allocated to the users having the best channels and spatial separation.

Precoding is an important component in MU MIMO/MISO systems as it allows spatial multiplexing in the downlink [26,27]. There are various linear and non-linear precoding strategies developed to improve the performance of $\mathrm{MU}$ MIMO/MISO systems. Furthermore, CSI at the BS is critical for precoding to achieve user multiplexing. Like SU systems, MU systems also suffer in the absence of perfect CSI at the transmitter. With imperfect CSI, precoding schemes at the BS cannot suppress the interference effectively at the user, caused by other users, therefore reducing the spectral efficiency of the system $[27,28]$. Interference is considered to be one of the limiting factors that reduces the spectral efficiency of MU systems. In a single cell MU communication system, we refer to the interference among the users as inter-user interference (IUI).

\subsubsection{Multicell Systems}

In addition to IUI, there is another type of interference known as out of cell interference. In cellular communication systems, increasing power of the BS increases the level of interference to other cells, especially in the $4 \mathrm{G}$ networks where the frequency reuse factor is one [1]. We refer to out of cell interference 
as inter-cell interference (ICI).

Despite the gains provided by the MIMO technology in the single-cell MU system, in practice the users close to the cell-edge are more susceptible to ICI, hence degrading the spectral efficiency of the cell. These systems are also known as multicell systems. In multicell MIMO [29-31], serving and neighboring base stations together contribute in enhancing the performance and reducing ICI. The coordination allows base stations to share the information (such as CSI) related to coordination via a backhaul link. Like MU systems, the quality of CSI at the BS also plays an important role in the performance of multicell MU systems.

\subsubsection{Massive Multi-antenna Systems}

Massive multi-antenna systems use large numbers of transmit antennas at the base station [32] and a relatively smaller numbers of antenna at the user, resulting in a higher spectral efficiency, less inter-user interference and reduced energy consumption [32-34]. The use of large numbers of antennas at the BS also provides highly directional beamforming [1]; the array gain from beamforming could improve the link budget. Due to these and several other attractive features, massive MISO is becoming a popular contender for $5 \mathrm{G}$ wireless systems. However, there are a number of factors that limit the performance of massive MISO systems, e.g. pilot contamination [34,35], reduced MU diversity due to channel hardening [36] and high spatial correlation at the BS [37]. An overview of massive MIMO is described in [32] including information theoretic aspects and linear transceivers along with the main design features and practical challenges. The attractive features of massive MIMO systems also apply to massive MISO systems. Like conventional (small-scale) multi-antenna systems, massive multi-antenna systems provide significant performance gains when CSI is available at the transmitter. However, in massive multi-antenna systems, the resources required to obtain CSI at the transmitter increase proportionally to the number of transmit antennas. Time division duplexing (TDD) communication is preferred for massive MIMO systems, but for systems with the imperfect RF chain calibration, limited feedback schemes can be used to equip the transmitter with CSI.

\subsection{Limited Feedback Techniques}

From the above discussion, it is clear that in order for MIMO wireless communication systems to provide further improvements in the performance, the transmitter must be able to adapt to changing channel conditions [20]. For this purpose, the transmitter requires some form of CSI. It is not practically possible 
to have perfect CSI at the transmitter, in fact CSI at the transmitter incorporates errors such as estimation and quantization, depending on a duplexing method. In TDD transmission, where forward and reverse links usually experience the same fading distribution, CSI can be obtained at the transmitter using channel reciprocity. To accomplish reciprocity in TDD systems, the transmitter and the receiver must have tight $\mathrm{RF}$ chain calibration [38-40]. However, in frequency division duplexing (FDD) communication, the forward and reverse links are uncorrelated due to different operating frequencies [20]. Therefore, limited feedback techniques are used in FDD MIMO systems to equip the transmitter with CSI; such systems are referred to as closed-loop MIMO. As the focus of this thesis is on limited feedback techniques, we restrict the further discussion to FDD based limited feedback multiple antenna systems.

In limited feedback MIMO systems, the receiver estimates the channel and selects an appropriate codebook entry or codeword and feeds back the index of that codeword to the transmitter. For this purpose, both transmitter and receiver maintains a common codebook. At the receiver, the mismatch between the estimated CSI and the selected codeword gives rise to codebook quantization error. Due to bandwidth limitations, a low-rate feedback link is commonly used by the receiver to send the index of the appropriate codeword to the transmitter. The number of bits required to send the index of the selected codeword to the transmitter is considered as a feedback overhead and it increases with the number of transmit antennas in MISO systems. Feedback bits are sent to the transmitter using an existing low-rate link for control signals. This link is well protected from errors by using error correcting codes and high transmission power. The receiver feeds back CSI frequently so that transmitter can adapt to the changing channel. The performance of limited feedback MIMO systems heavily depend on the codebook design and the quality of the CSI at the transmitter.

Efficient limited feedback schemes aim to minimize the quantization errors by employing effective codebooks and feedback strategy. Limited feedback techniques have shown to be effective in providing CSI at the transmitter for both SU and MU systems. The quantized CSI at the transmitter degrades the performance compared to the perfect CSI, but the performance with the quantized CSI is still better than the case where no CSI is available at the transmitter.

Limited feedback techniques are part of $4 \mathrm{G}$ wireless communications standards such as third generation partnership program (3GPP) long term evolution (LTE) and IEEE $802.16 \mathrm{~m}$ standards. In order to meet the requirement of the International Telecommunication Union (ITU) for International Mobile Telecommunications advanced (IMT-Advanced) 4G technologies [41], both standards support closed-loop MIMO communication in SU and MU transmissions. The codebooks developed for LTE and IEEE 802.16m standards are different, 
targeting different antenna configurations and channel characteristics. Network coordinated and massive MIMO are an integral part of beyond $4 \mathrm{G}$ systems, such as $5 \mathrm{G}$ cellular systems.

\subsection{Research Framework}

This section discusses the motivation and the main contributions of the thesis.

\subsubsection{Motivation}

One of the major factors influencing codebook design in limited feedback systems is the propagation environment. The research on designing a codebook for Rayleigh independent and identically distributed (i.i.d.) channels $[22,42,43]$ has dominated for many years. In practice, MIMO communication channels are spatially/temporally correlated. Codebooks such as the Grassmannian and LTE (designed for Rayleigh i.i.d. channels) do not perform well in correlated channels [44]. The drawback of these codebooks are that they are fixed and cannot adapt to changing channel conditions. In the recent past numerous codebook designs [44-46] have been developed for correlated channels. Depending on a level of spatial and temporal correlation, a codebook can be maneuvered and transformed accordingly after each feedback interval. In this thesis, we refer to such codebooks as differential codebooks.

Recent work on differential codebooks and their importance in next generation of wireless systems motivate us to develop codebooks for MISO systems that exploit spatial and temporal correlation in the channel. By considering the correlation statistics of the channel in a codebook design, the quality of CSI at the transmitter can be improved significantly.

In temporally correlated scenarios, the channel entries do not change abruptly over time. Differential codebooks take advantage of this property and transform the codewords such that they lie close to the previously selected codeword. Hence, enabling the differential codebook to track the slow varying channel. Furthermore, spatial correlation restricts the variation of the channel towards the direction of the eigenvectors of the transmit correlation matrix. Therefore, by taking into account the effects of correlation, differential codebooks with effective adaptive scaling and rotation techniques improve CSI quality at the transmitter and minimize codebook quantization errors [46].

Recently, massive MISO has been widely investigated $[47,48]$ with limited feedback techniques in FDD based systems. The codebook-based methods are practically infeasible for massive MIMO systems due to the exhaustive search required for selecting the appropriate codeword. This issue of codebook impracticality motivates us to design an alternate limited feedback scheme in temporally 
correlated channels for massive MISO systems with a lower search complexity.

In coordinated multicell systems, adaptive bit allocation methods [49-52] have received enormous attention recently. A typical adaptive bit allocation method divides the total number of feedback bits between the desired and out-ofcell interfering channels by maximizing/minimizing a specific performance/distortion metric. Motivated by existing adaptive bit allocation methods using different precoding schemes, we investigate an adaptive bit allocation scheme for the coordinated regularized zero-forcing (RZF) precoding.

\subsubsection{Contributions}

In order to make practical and productive use of MIMO technology, limited feedback schemes with effective codebook designs are essential to minimize CSI quantization errors. In this thesis, different aspects of limited feedback schemes are examined. Throughout the thesis multi-antenna downlink systems are considered.

We begin by investigating capacity gains of MIMO systems over single-input single-output (SISO) systems with perfect CSI and no CSI at the transmitter. With imperfect CSI, we analyze the capacity loss due to codebook quantization errors using well-known codebooks for SU systems with three different MIMO receivers. After considering SU MIMO capacity loss analysis, the focus of the research shifts towards the design of differential codebooks for MU MISO systems in temporally/spatially correlated channels. We investigate various differential codebook designs, that quantize channel direction information (CDI), by exploiting temporal/spatial correlation in the channel and transforming the codebook after each feedback interval. We also consider massive MU MISO systems with limited feedback to acquire CDI at the transmitter, where we design an effective limited feedback strategy that reduces the search complexity while quantizing temporally correlated channels at the receiver. Finally, we study limited feedback in multicell MU MISO systems for Rayleigh i.i.d. channels and develop a coordinated regularized zero-forcing (RZF) precoding scheme for conventional (small-scale) MU MISO systems.

The main contributions of the thesis are listed below:

- MIMO capacity analysis: The contributions of MIMO capacity gain and MIMO capacity loss analysis for SU systems are:

- Investigation of capacity gains of the MIMO system over the SISO system using various analytical channel models with perfect CSI and no CSI at the transmitter.

- Derivation of capacity loss approximations in terms of codebook quantization errors for SU MIMO systems with multilayer transmis- 
sion and using three different MIMO receivers, namely: zero forcing (ZF), minimum mean-squared error (MMSE) and singular value decomposition (SVD).

- Evaluation of the capacity loss using 3GPP LTE and Grassmannian codebooks and three different MIMO channel models: i.i.d. Rayleigh channel, spatially correlated Rayleigh channel and industry standard based WINNER II channel.

- MU MISO systems with differential codebooks: We study two differential codebook structures for single-cell MU MISO systems operating under spatially and temporally correlated channels. The two types of differential codebook designs are based on single-cap (spherical) and double-cap (spherical and polar) structures, where a cap is a spherical region on a sphere with a specific radius. The main contributions of the work are given below:

Spherical cap differential codebook:

- Introduction of an SVD based rotation scheme that effectively rotates the codewords in the codebook to the desired position without errors.

- In order to successfully track the slow varying channel, two adaptive scaling techniques are developed: Adaptive Scaling Technique I and Adaptive Scaling Technique II.

Double cap differential codebook:

- Construction of combined spherical cap and polar cap based differential codebook.

- Development of the blind adaptive scaling technique that leverage the information inherent in a two cap structure to enable scaling up and scaling down of the cap radii

- MU massive MISO systems with differential trellis coded quantization (TCQ): We investigate MU massive MISO systems with limited feedback in temporally correlated channels. The main contributions are:

- Development of an efficient differential TCQ method that quantizes temporally correlated channels at the receiver with a lower search complexity than that of conventional codebook schemes.

- Transformation of the TCQ source constellation by using 2D translation and scaling techniques.

- Derivation of a scaling factor for the TCQ source constellation as a function of the number of BS antennas and the temporal correlation. 
- Development of a TCQ based limited feedback scheme for spatially correlated massive MISO channels using uniform linear array (ULA) and uniform rectangular array (URA) antenna topologies.

- Coordinated multicell MU MISO systems: We study coordinated multicell MU MISO systems for i.i.d. Rayleigh channels with limited feedback. The contributions of this work are summarized as follows:

- Development of a coordinated RZF precoding strategy where the serving BS of the cell shares out-of-cell interfering channels with the respective interfering base stations to minimize interference in the network.

- Derivation of expected signal-to-interference-plus-noise ratio (SINR) approximations for coordinated RZF scheme with both perfect CDI and limited feedback based quantized random vector quantization (RVQ) CDI.

- Development of an adaptive feedback bit allocation strategy that minimizes the interference in the network.

\subsubsection{List of Publications}

The content of this thesis has been partially published, accepted or submitted for publication in the papers listed below.

1. M. Zhang, P. J. Smith, M. Shafi, P. A. Dmochowski, J. Mirza, "MIMO Capacity Gain Analysis for General Channel Models", in Proc. IEEE International Conference on Communications (ICC), pp. 4846-4851, 2012.

2. J. Mirza, P. A. Dmochowski, P. J. Smith, M. Shafi, "Capacity Loss for Multilayer Codebook Precoding in MIMO Systems", in Proc. 23rd IEEE International Symposium on Personal, Indoor and Mobile Radio Communications (PIMRC), pp. 1890-1895, 2012.

3. J. Mirza, P. A. Dmochowski, P. J. Smith, M. Shafi, "Limited feedback multiuser MISO systems with differential codebooks in correlated channels", in Proc. IEEE International Conference on Communications (ICC), pp. 5386-5391, 2013.

4. J. Mirza, P. A. Dmochowski, P. J. Smith, M. Shafi, "A differential codebook with adaptive scaling for limited feedback MU MISO systems", IEEE Wireless Communications Letters, pp. 2-5, 2014. 
5. J. Mirza, P. J. Smith, M. Shafi, P. A. Dmochowski, A. Firag, A. Papathanassiou, "Double-Cap Differential Codebook Structure for MU MISO Systems in Correlated Channels", IEEE Wireless Communications Letters, pp. 441-444, 2014.

6. J. Mirza, M. Shafi, P. J. Smith, P. A. Dmochowski, "Limited Feedback Massive MISO Systems with Trellis Coded Quantization for Correlated Channels", accepted in IEEE Transactions on Vehicular Technology, 2015.

7. J. Mirza, P. J. Smith, P. A. Dmochowski, M. Shafi, "Coordinated Regularized Zero-Forcing Precoding for Multicell MISO Systems with Limited Feedback", submitted to IEEE Transactions on Vehicular Technology, 2015.

\subsection{Thesis Outline}

The rest of the dissertation is organized as follows:

Chapter 2 introduces the theoretical background of MIMO channel models that are used in this thesis. The MIMO channel models are classified into two categories: analytical and standardized MIMO channel models.

Chapter 3 provides an overview of basic concepts and background theory in limited feedback MIMO systems.

Chapter 4 gives the capacity loss analysis of the SU MIMO limited feedback system using LTE and Grassmannian codebooks. This chapter begins with a brief description of MIMO capacity gains without limited feedback. Later, in the capacity loss analysis, single stream and two stream MIMO transmission modes are considered. The capacity loss expressions are also derived using different MIMO receivers, followed by numerical results and discussion.

Chapter 5 investigates differential codebook designs for spatially/temporally correlated MU MISO channels. This chapter is divided into two categories: single spherical-cap and double-cap differential codebooks. Various adaptive scaling techniques are discussed for both differential codebook designs. An SVD based codebook rotation method is discussed. Simulation results are also presented for single spherical-cap and double-cap differential codebooks.

Chapter 6 focuses on massive MU MISO systems with limited feedback. As motivation, the impracticality of RVQ codebooks is discussed. Later, a differential TCQ method is presented to quantize the channel (at the user) and reconstruct the channel (at the $\mathrm{BS}$ ) in temporally correlated channels. The $2 \mathrm{D}$ translation and scaling techniques for differential TCQ are also presented. A scaling parameter that is a function of the number of transmit antennas and temporal correlation is derived for the source constellation. A TCQ based lim- 
ited feedback scheme for spatially correlated channels is investigated, followed by numerical results and discussion.

Chapter 7 studies coordinated multicell MU MISO systems in Rayleigh i.i.d. fading channels. First, coordinated RZF scheme is described, followed by the expected SINR analysis for both perfect CDI and RVQ based imperfect CDI. An adaptive bit allocation strategy is also presented in this chapter. Finally, simulation results are presented for the coordinated RZF precoding scheme.

Chapter 8 draws conclusions from the contributions of the thesis and suggests future research directions. 


\section{2 \\ Wireless Channels}

An overview of MIMO channels is presented in this chapter. First, some key concepts of wireless channels are discussed, followed by a detailed explanation of analytical and standardized MIMO channel models used in this thesis. We also discuss the capacity of MIMO wireless channels assuming that perfect CSI is available at the receiver.

\subsection{Wireless Radio Channel}

It is important to understand the characteristics of wireless communication channels, as they generally dictate the performance of wireless systems.

In a typical wireless communication system, the variation in the power of the received signal with respect to distance is generally due to path loss and shadowing [23]. These variations usually occur at large distances, and are thus also referred to as large-scale propagation effects. On the other hand, multipath where a signal reaches the receiver from two or more paths (and adds up constructively or destructively) is termed small-scale propagation effects [23]. The effects of large-scale and small-scale fading are illustrated in Fig. 2.1, where $P_{t}$ is the transmit signal power, $P_{r}$ is the receive signal power and the distance between the transmitter and receiver is denoted by $d$.

The well-known free-space path loss model is commonly used when there is no obstruction in the path of the signal [23]. Also, there are numerous raytracing propagation models developed when the geometry of the region is known and there are few multipath components [23]. In addition to free space path loss and ray-tracing models, there are some empirical path loss models that are developed to estimate the path loss in the typical wireless communication 


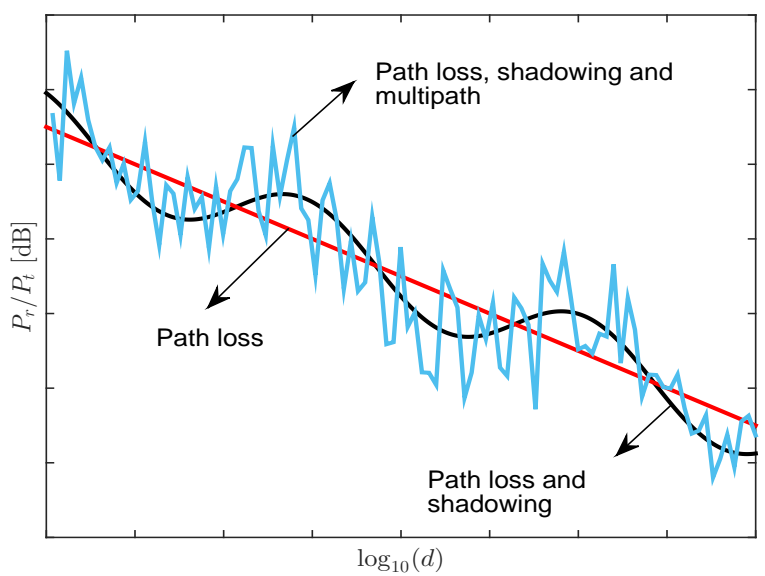

Figure 2.1: Effects of path loss, shadowing and multipath on $P_{r} / P_{t}(\mathrm{~dB})$ ratio with log distance [23].

environments such as urban macro (UMa), urban micro (UMi), outdoor-toindoor (O2I) and a few others. Empirical path loss models are developed by Okumara, Hata, COST 231, SUI, LTE and many other studies.

In this thesis, we consider a combined path loss and shadowing model for evaluating the performance of cellular systems. The received signal power with the combined path loss and shadowing model is written in linear scale as [24]

$$
P_{r}=P_{0}\left(\frac{R_{c}}{d}\right)^{a} \Phi
$$

where $P_{0}$ is the received power at the radius $R_{c}$ without shadowing, and $a$ is a path loss exponent. The shadowing is modeled as a log-normal random variable, given by $\Phi=10^{\left(\eta \sigma_{\mathrm{SF}} / 10\right)}$, where $\sigma_{\mathrm{SF}}$ is the shadowing standard deviation in $\mathrm{dB}$ and $\eta$ is a zero mean Gaussian random variable with unit variance. The distance $d$ represents the distance of the receiver from the transmitter.

For any SISO link, the channel impulse response (CIR) of the time-varying fading multipath channel is given by [24]

$$
h(\tau, t)=\sum_{i} \alpha_{i}(t) \delta\left(\tau-\tau_{i}(t)\right)
$$

where $t$ and $\tau$ denote time and delay, respectively. $\alpha_{i}$ is the overall attenuation and $\tau_{i}$ is the propagation delay at time $t$ for the path $i$, from the transmitter to the receiver.

Now, we briefly discuss the concept of time-frequency coherence in a wireless communication channel. The coherence time, $T_{c}$, of the channel is inversely proportional to the Doppler spread, $D_{s}$, such that, $T_{c}=1 / 4 D_{s}[24]$. If the carrier frequency is denoted by $f_{c}$, then the Doppler spread is defined as the 
maximum difference between the Doppler shifts of paths $i$ and $j$, such that

$$
D_{s}:=\max _{i, j} f_{c}\left|\tau_{i}(t)-\tau_{j}(t)\right|
$$

When the coherence time, $T_{c}$ is smaller than the delay requirement of the application, $T_{\text {req }}$, the channel is considered to be a fast fading channel and a slow fading channel if $T_{c}$ is larger than $T_{\text {req }}[24]$.

Another key time-frequency coherence parameter of the wireless communication channel is the coherence bandwidth, $B_{c}$, defined as $B_{c}=1 / 2 T_{d}$ [24], where $T_{d}$ is the delay spread given by

$$
T_{d}:=\max _{i, j}\left|\tau_{i}(t)-\tau_{j}(t)\right|
$$

A channel is considered to be flat fading when $B_{c}$ is much greater than the bandwidth of the signal, $B_{s}$, i.e., $B_{c} \gg B_{s}$ and frequency selective fading when $B_{c} \ll B_{s}$. Table 2.1, shows the types of wireless channels. In wideband channels,

Table 2.1: Types of wireless channels [24].

\begin{tabular}{c|c}
\hline Type & Definition \\
\hline Fast fading & $T_{c} \ll T_{\text {req }}$ \\
Slow fading & $T_{c} \gg T_{\text {req }}$ \\
Flat fading & $B_{s} \ll B_{c}$ \\
Frequency selective fading & $B_{s} \gg B_{c}$ \\
\hline
\end{tabular}

multipath components are resolvable as the delay spread of the channel is greater than the inverse of the bandwidth of the signal, i.e., $T_{d} \gg B_{s}^{-1}$. On the contrary, in narrowband channels multipath components are non-resolvable as $T_{d} \ll B_{s}^{-1}$. Therefore, in narrowband channels, we have $h_{n_{r}, n_{t}}(t, \tau) \approx h_{n_{r}, n_{t}}(t)$.

The discussion so far only considered a SISO link. For MIMO channels with $n_{t}$ transmit and $n_{r}$ receive antennas, the wideband MIMO channel matrix, $\mathbf{H}(t, \tau)$ of size $n_{r} \times n_{t}$ is written as

$$
\mathbf{H}(t, \tau)=\left[\begin{array}{cccc}
h_{1,1}(t, \tau) & h_{1,2}(t, \tau) & \cdots & h_{1, n_{t}}(t, \tau) \\
h_{2,1}(t, \tau) & h_{2,2}(t, \tau) & \cdots & h_{2, n_{t}}(t, \tau) \\
\vdots & \vdots & \ddots & \vdots \\
h_{n_{r}, 1}(t, \tau) & h_{n_{r}, 2}(t, \tau) & \cdots & h_{n_{r}, n_{t}}(t, \tau)
\end{array}\right]
$$

where $h_{n_{r}, n_{t}}(t, \tau)$ is a CIR between the $n_{t}^{\text {th }}$ transmit and the $n_{r}^{\text {th }}$ receive antenna.

Most of the channels used in this thesis are narrowband channels. However, when evaluating capacity loss for MIMO orthogonal frequency-division multiplexing (OFDM) channels in Chapter 3, we use wideband channels. In the next two sections, we discuss analytical and standardized MIMO channel models. 


\subsection{Analytical MIMO Channel Models}

Analytical channel models, also referred as stochastic channel models give the statistical representation of the channel. Although, they do not fully match real-world channels, they are widely used to evaluate the performance of MIMO systems. In this section, we discuss four kinds of analytical MIMO channel models, namely, Rayleigh i.i.d., Rician, spatially correlated and temporally correlated channel models.

\subsubsection{Rayleigh i.i.d. Channel}

The Rayleigh i.i.d. Channel is the simplest statistical MIMO channel model for rich scattering and non-line-of-sight (NLoS) environments [3]. In Rayleigh i.i.d. MIMO channels, each link is assumed independent and complex random variable with Rayleigh distributed amplitude and uniformly distributed phase [1]. This model is practically suitable when the spacing between the antenna elements is sufficiently large and angular spreads are large (rich scattering) at the transmitter and the receiver. The entries of i.i.d. Rayleigh MIMO channel matrix, $\mathbf{H}$, are independent and distributed according to $\mathcal{C N}(0,1)$. Without considering path loss and shadowing, the i.i.d. Rayleigh MIMO channels follow

$$
\mathbb{E}\left[\|\mathbf{H}\|_{F}^{2}\right]=n_{t} n_{r}
$$

In this thesis, we extensively use MISO channels where the receiver has only one antenna. In such cases, Rayleigh i.i.d. MISO channel, $\mathbf{h}$, is a vector of size $1 \times n_{t}$, where $\mathbb{E}\left[\|\mathbf{h}\|_{2}^{2}\right]=n_{t}$.

\subsubsection{Rician Channel}

Rician channel models are used in the scenarios where there exists a strong lineof-sight (LoS) path between the transmitter and the receiver. The LoS path is independent of fading [1], therefore it is not modeled as a zero-mean random variable [23]. The Rician channel consists of both LoS and NLoS components and it is modeled as

$$
\mathbf{H}_{\text {Rician }}=\sqrt{\frac{K_{f}}{1+K_{f}}} \mathbf{H}_{\mathrm{LoS}}+\sqrt{\frac{1}{1+K_{f}}} \mathbf{H}
$$

where $\mathbf{H}_{\mathrm{LoS}}$ is the matrix containing LoS components. The entry $(i, j)$ of the $\mathbf{H}_{\mathrm{LoS}}$ can be expressed as $\mathrm{e}^{j \theta_{i, j}}$, where $\theta_{i, j}$ is the uniform phase. $\mathbf{H}$ is the Rayleigh i.i.d. channel and $K_{f}$ is the so-called $\mathrm{K}$ factor. The $\mathrm{K}$ factor is defined as the ratio of the energy in the LoS component to the energy in NLoS components [24]. 
When $K_{f}=0$, we have the Rayleigh i.i.d. channel in (2.7) and for $K_{f}=\infty$ we get only the LoS component. The distribution of the magnitude of the channel entries in (2.7) is Rician.

\subsubsection{Spatially Correlated Rayleigh Channel}

For spatially correlated Rayleigh MIMO channels, we rely on the separable Kronecker model $[53,54]$, where correlation matrices are used to express the spatially correlated Rayleigh MIMO channel as

$$
\hat{\mathbf{H}}=\mathbf{R}_{\mathrm{r}}^{1 / 2} \mathbf{H R}_{\mathrm{t}}^{1 / 2}
$$

where $\mathbf{R}_{\mathrm{r}}$ and $\mathbf{R}_{\mathrm{t}}$ are the receive and transmit correlation matrices, respectively. $\mathbf{H}$ is the i.i.d. Rayleigh MIMO channel. This model is useful when the spacing between the antenna elements is small either at the transmitter, the receiver, or both. The underlaying channel in (2.8) is the i.i.d. Rayleigh channel which assumes independent and rich scattering at both ends, therefore making it possible to separate the transmit and receive correlations. In this thesis, the entries of correlation matrices $\mathbf{R}_{\mathrm{t}}$ and $\mathbf{R}_{\mathrm{r}}$ follow an exponential model [55], given by

$$
r_{\mathrm{t}}^{i j}=z_{\mathrm{t}}^{d^{i j}}
$$

and

$$
r_{\mathrm{r}}^{i j}=z_{\mathrm{r}}^{d^{i j}}
$$

respectively, where $d^{i j}$ is the distance between the antenna $i$ and $j$. $z_{\mathrm{t}}$ and $z_{\mathrm{r}}$ are the transmit and receive correlation coefficients. The magnitude of $z_{\mathrm{t}}$ and $z_{\mathrm{r}}$ varies from 0 to 1 , where 0 represents no spatial correlation and 1 means full correlation, i.e., the same fading on both links. In a MISO channel, (2.8) simplifies to

$$
\hat{\mathbf{h}}=\mathbf{h} \mathbf{R}_{\mathrm{t}}^{1 / 2} .
$$

where $\mathbf{h}$ is the i.i.d. Rayleigh MISO channel. The exponential correlation model has shown to be reasonable for the equally-spaced linear antenna arrays, but it may not be a valid model for some real-world scenarios. However, the exponential model is a simple model which is helpful in studying the effect of correlation on the MIMO capacity and also provides some useful insights [55].

\subsubsection{Temporally Correlated Rayleigh Channel}

Most of the work done in this thesis considers temporally correlated channels. When the receiver is moving slowly, its channel entries vary slowly over time. A first-order Gauss-Markov (FOGM) filter, is used to model the temporally 
correlated MIMO channel at time $t$ as $[24,56]$

$$
\tilde{\mathbf{H}}[t]=\epsilon \tilde{\mathbf{H}}[t-1]+\sqrt{1-\epsilon^{2}} \mathbf{Y}[t],
$$

where $\epsilon$ is a temporal correlation coefficient that follows Jakes' model (which is an isotropic scattering leading to Bessel function), such that, $\epsilon=J_{0}\left(2 \pi f_{d} T_{s}\right)$, where $J_{0}$ is a Bessel function of zeroth order, $f_{d}$ is the maximum Doppler frequency and $T_{s}$ is the channel sample time. $\mathbf{Y}[t]$ is an innovation process with its entries distributed according to $\mathcal{C N}(0,1)$. For fast moving receivers, $\epsilon$ is close to or equal to zero and for slow moving receivers the $\epsilon$ value is close to one.

For temporally correlated MISO channels, we have

$$
\tilde{\mathbf{h}}[t]=\epsilon \tilde{\mathbf{h}}[t-1]+\sqrt{1-\epsilon^{2}} \mathbf{y}[t] .
$$

A combined spatially and temporally correlated MISO channel can also be modeled as $[1,46]$

$$
\overline{\mathbf{h}}[t]=\epsilon \overline{\mathbf{h}}[t-1]+\sqrt{1-\epsilon^{2}} \mathbf{y}[t] \mathbf{R}_{\mathrm{t}}^{1 / 2} .
$$

In this thesis, we have extensively used analytical MIMO/MISO channel models, however we also used standardized MIMO channel models in order to evaluate the performance of the developed methods and schemes under more realistic propagation environments.

\subsection{Standardized MIMO Channel Models}

Several standards for MIMO channels are developed by various international organizations including 3GPP and IMT-Advanced [57]. These standards are sometimes extended in order to improve spatial channel modeling by supporting more MIMO technologies and propagation scenarios. Mostly, the standards follow geometry-based stochastic channel modeling approaches, where statistics of the key channel parameters are estimated using real-time channel measurements. In this section, two standards are discussed, namely, 3GPP spatial channel model (SCM) and Wireless World Initiative New Radio (WINNER) channel models.

\subsubsection{GPP Spatial Channel Model}

3GPP SCM [58] has been designed for outdoor scenarios with system bandwidth of $5 \mathrm{MHz}$, operating at around $2 \mathrm{GHz}$ carrier frequency. The outdoor scenarios supported by the SCM model are UMa (only NLoS), UMi (both LoS and NLoS) and suburban macro (SMa) (only NLoS). The statistics of channel parameters are obtained from real-world channel measurements. Polarized antennas are also 
included in 3GPP SCM. Due to lack of scenarios considered and few LoS Rician K-factor results, 3GPP SCM model has been extended by SCME (extendedSCM). SCME supports bandwidths up to $100 \mathrm{MHz}$ and carrier frequency of 5 GHz. This model aids both LoS and NLoS environments in UMa, UMi and SMa scenarios. Both SCM and SCME lack some features such as indoor scenarios, LoS measurements, elevation angles and time evolution.

\subsubsection{WINNER Channel Models}

WINNER channel models target beyond $3 \mathrm{G}$ wireless systems with higher bandwidths and operating frequencies. This model includes both outdoor and indoor scenarios. Apart from additional scenarios, it also includes more results on LoS Rician K-factor. The WINNER project introduced WINNER I channel models initially and those were later extended by WINNER II channel models. Finally, WINNER + channel models have been developed which are evolved from WINNER I and WINNER II channel models.

WINNER I [59] supports 7 outdoor/indoor scenarios and introduce two types of models: geometric-based stochastic model and reduced variability (cluster delay line) model. The geometric-based stochastic model allows the generation of a double directional propagation channel which is antenna independent and supports multi-links. In the WINNER I project, measurements are performed at $2 \mathrm{GHz}$ and $5 \mathrm{GHz}$ carrier frequencies with $100 \mathrm{MHz}$ bandwidth. The channel parameters investigated are power delay profile, path loss, shadowing, delay spreads, angle spreads and cross-polarization ratio (XPR). The model also provides correlation between large-scale parameters WINNER II channel models [60] for link and system level simulations are evolved from WINNER I. It supports 11 outdoor and indoor scenarios. Apart from new scenarios, this model also features steady time and space evolution. The model is scalable from a single-cell SU/MU SISO/MIMO to SU/MU multicell MIMO. Both fixed and moving relay stations are supported by WINNER II channel models. Some additional features include modeling of elevation rays and moving scatterers. WINNER II channels have been the starting point for the IMT-Advanced based channel model (M.2135) recommended by ITU-R.

WINNER + channel models [61] are developed to support IMT-Advanced technologies like 3GPP LTE-Advanced. WINNER + upgrades 2D channel models to 3D channel models by specifying elevation angles, and large-scale and small-scale parameters for the elevation domain. For the elevation domain, large-scale and small-scale parameters are assumed to have normal and Laplace distributions, respectively. This model is applicable to wireless systems operating in $450 \mathrm{MHz}$ to $6 \mathrm{GHz}$ frequency range.

This thesis considers a standardized MIMO channel based on the WINNER 
II channel models [60]. The single link between the transmitter and the receiver in the WINNER II channel model is shown in Fig. 2.2.

The single link model consists of a finite number of clusters and each cluster has a fixed number of rays. For example, the number of clusters for UMa scenario is 20 , whereas, for rural macro (RMa) propagation scenario the number of clusters is 10. For both the scenarios, the number of rays in each cluster is 30 . Each ray has its own angle of departure (AoD) and angle of arrival (AoA). All the

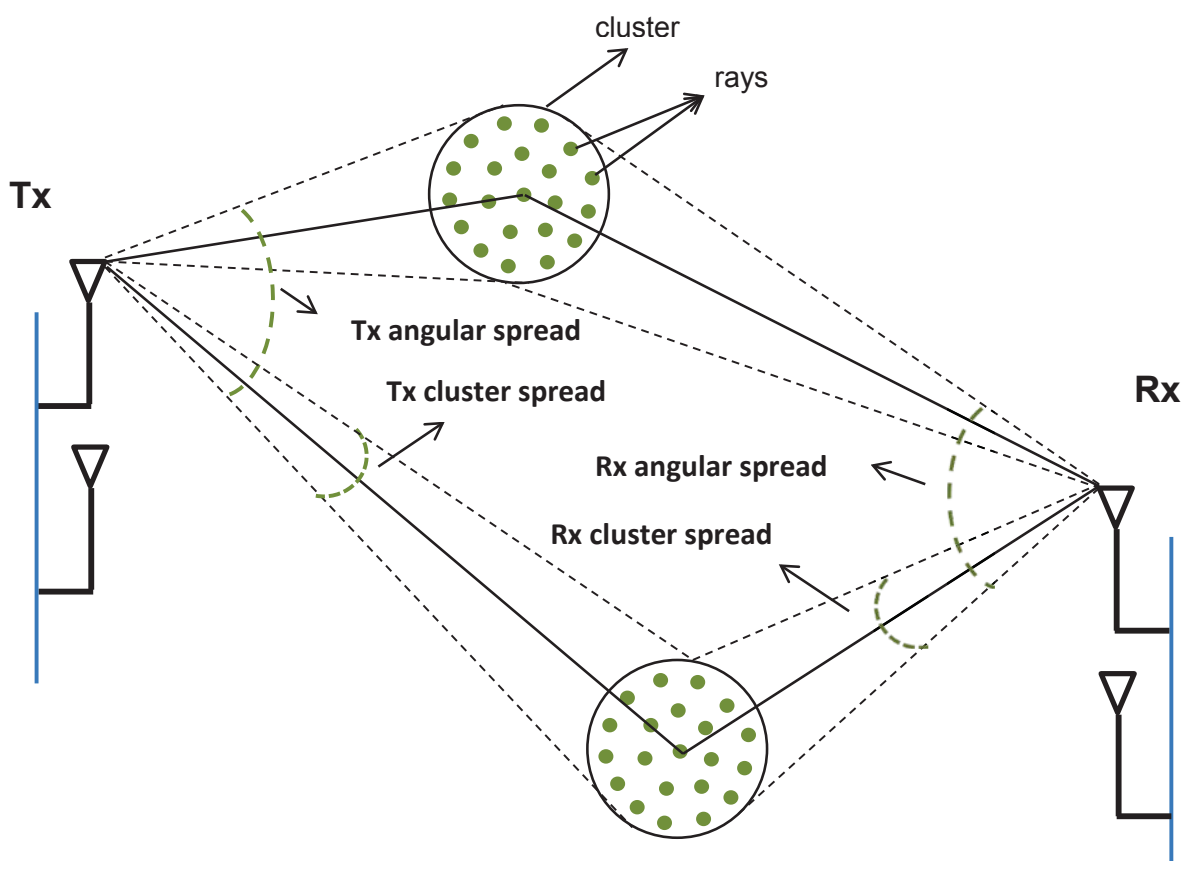

Figure 2.2: The single link between the transmitter and the receiver in the WINNER II channel model.

Table 2.2: WINNER II MIMO channel model parameters

\begin{tabular}{|c|c|c|c|c|}
\hline \multicolumn{2}{|c|}{ Parameters } & UMa & RMa & 02I-UMa \\
\hline \multirow{2}{*}{ Delay, $\tau$, spread $\log _{10}(\mathrm{~s})$} & $\mu$ & -6.63 & -7.60 & -6.62 \\
\cline { 2 - 6 } & $\sigma$ & 0.32 & 0.48 & 0.32 \\
\hline \multirow{2}{*}{ Azimuth AoD, $\phi$, spread $\log _{10}($ degree$)$} & $\mu$ & 0.93 & 0.96 & 1.76 \\
\cline { 2 - 6 } & $\sigma$ & 0.22 & 0.45 & 0.16 \\
\hline \multirow{2}{*}{ Azimuth AoA, $\varphi$, spread $\log _{10}($ degree$)$} & $\mu$ & 1.72 & 1.52 & 1.25 \\
\cline { 2 - 6 } & $\sigma$ & 0.14 & 0.27 & 0.42 \\
\hline Cross-polarization ratio (XPR) (dB) & $\mu$ & 7 & 7 & 9 \\
\cline { 2 - 6 } & $\sigma$ & 3 & 4 & 11 \\
\hline Number of clusters & 20 & 10 & 12 \\
\hline Delay distribution & \multicolumn{3}{c}{ EXP } \\
\hline Azimuth AoA and AoD distribution & & \multicolumn{3}{c}{ Wrapped Gaussian } \\
\hline
\end{tabular}


clusters in the link have different power and delay values associated with them. The WINNER II channel model incorporates various antenna configurations such as co-polarized, dual polarized and group polarized. Benefit of using the WINNER II channel model is that it can be used to generate both narrowband and wideband communication channels.

The parameters of WINNER II channels for UMa, RMa, and O2I-UMa scenarios under the NLoS propagation are summarized in Table 2.2.

In this thesis, MATLAB simulation of the WINNER II channel model in [62] are used to generate the MIMO channel.

\section{Narrowband WINNER II channel}

The narrowband WINNER II channel coefficient between the $i^{\text {th }}$ receive antenna and the $k^{\text {th }}$ transmit antenna, for the $n^{\text {th }}$ cluster is given by

$$
\begin{aligned}
h(i, k, n, t)= & \sqrt{P_{n}} \sum_{m=1}^{M_{r}}\left[\begin{array}{c}
F_{i, V}\left(\varphi_{n, m}\right) \\
F_{i, H}\left(\varphi_{n, m}\right)
\end{array}\right]^{T}\left[\begin{array}{cc}
\mathrm{e}^{j \Theta_{n, m}^{V V}} & \sqrt{\varpi_{n, m}} \mathrm{e}^{j \Theta_{n, m}^{V H}} \\
\sqrt{\varpi_{n, m}} \mathrm{e}^{j \Theta_{n, m}^{H V}} & \mathrm{e}^{j \Theta_{n, m}^{H H}}
\end{array}\right] \\
& \times\left[\begin{array}{c}
F_{k, V}\left(\phi_{n, m}\right) \\
F_{k, H}\left(\phi_{n, m}\right)
\end{array}\right] \exp \left(j d_{k} 2 \pi \lambda_{0}^{-1} \sin \left(\phi_{n, m}\right)\right) \\
& \times \exp \left(j d_{i} 2 \pi \lambda_{0}^{-1} \sin \left(\varphi_{n, m}\right)\right) \exp \left(j 2 \pi v_{n, m} t\right),
\end{aligned}
$$

where:

- $M_{r}$ is the total number of rays in the cluster.

- $P_{n}$ is the power of the $n^{\text {th }}$ cluster.

- $F_{i, V}$ and $F_{i, H}$ are receive antenna field patterns for the vertical and horizontal polarizations respectively.

- $F_{k, V}$ and $F_{k, H}$ are transmit antenna field patterns for the vertical and horizontal polarizations respectively.

- $\Theta_{n, m}^{V V}, \Theta_{n, m}^{V H}, \Theta_{n, m}^{H V}$ and $\Theta_{n, m}^{H H}$ are initial random phases uniformly distributed from $-\pi$ to $\pi$, for the $n^{\text {th }}$ cluster and the $m^{\text {th }}$ ray.

- $\lambda_{0}$ is the wavelength of the carrier frequency.

- $\phi_{n, m}$ is AoD for the ray coming from the $n^{\text {th }}$ cluster and $m^{\text {th }}$ ray.

- $\varphi_{n, m}$ is AoA for the ray coming from the $n^{\text {th }}$ cluster and $m^{\text {th }}$ ray.

- $d_{k}$ and $d_{i}$ are the transmit antenna and the receive antenna spacing, respectively. 
- $v_{n, m}$ is the velocity component corresponding to the $m^{\text {th }}$ ray in the $n^{\text {th }}$ cluster.

- $\varpi_{n, m}$ is the cross polarization power ratio (XPR) corresponding to the $m^{\text {th }}$ ray in the $n^{\text {th }}$ cluster.

In order to generate a narrowband channel, the channel coefficients from different clusters are summed together. The resulting narrowband channel coefficient for any given time $t$ is

$$
h(i, k, t)=\sum_{n=1}^{N_{s}} h(i, k, n, t) .
$$

where $N_{s}$ is the total number of clusters. In (2.15), both spatial correlation and temporal correlation are present. The spatial correlation is induced by the transmit antenna spacing, $d_{k}$, and the receive antenna spacing, $d_{i}$, the smaller the spacings, the higher the spatial correlation in the channel and vice versa. On the other hand, the velocity component, $v_{n, m}$, controls the amount of temporal correlation in the channel, where higher the velocity, the lower the temporal correlation in the channel, and vice versa.

\section{Wideband WINNER II channel}

Unlike narrowband channels, the wideband channels consider the time dispersive nature of the communication channel. Therefore, the delay associated with each ray is required in order to model a wideband channel. The wideband channel coefficient, between the $i^{\text {th }}$ receive antenna and the $k^{\text {th }}$ transmit antenna, at any particular time $t$ with path delay $\tau$ is given by

$$
h(i, k, t, \tau)=\sum_{n=1}^{N_{s}} h(i, k, n, t, \tau),
$$

where the wideband channel for the $n^{\text {th }}$ cluster is given by

$$
\begin{aligned}
h(i, k, n, t, \tau) & =\sqrt{P_{n}} \sum_{m=1}^{M_{r}}\left[\begin{array}{c}
F_{i, V}\left(\varphi_{n, m}\right) \\
F_{i, H}\left(\varphi_{n, m}\right)
\end{array}\right]^{T}\left[\begin{array}{cc}
\mathrm{e}^{j \Theta_{n, m}^{V V}} & \sqrt{\varpi_{n, m}} \mathrm{e}^{j \Theta_{n, m}^{V H}} \\
\sqrt{\varpi_{n, m}} \mathrm{e}^{j \Theta_{n, m}^{H V}} & \mathrm{e}^{j \Theta_{n, m}^{H H}}
\end{array}\right] \\
& \times\left[\begin{array}{l}
F_{k, V}\left(\phi_{n, m}\right) \\
F_{k, H}\left(\phi_{n, m}\right)
\end{array}\right] \exp \left(j d_{k} 2 \pi \lambda_{0}^{-1} \sin \left(\phi_{n, m}\right)\right) \\
& \times \exp \left(j d_{i} 2 \pi \lambda_{0}^{-1} \sin \left(\varphi_{n, m}\right)\right) \exp \left(j 2 \pi v_{n, m} t\right) \delta\left(\tau-\tau_{n, m}\right) .
\end{aligned}
$$

In Chapter 4, we generate a wideband MIMO channel for an OFDM system using (2.17) assuming all the rays within the cluster have same delay. The 
channel frequency response is required in wideband MIMO channels, in order to solve for the cluster delay values.

In this thesis, two basic antenna configurations, namely: co-polarized and dual polarized are considered. We consider co-polarized antennas where dipole antennas are mounted vertically (single polarization). For the dual polarized antenna case, we consider a pair of dipole antennas which are vertical/horizontal arrays having $+/-45$ degree slant orientation.

\subsection{Capacity of MIMO Wireless Channels}

In this section, we discuss the capacity of SU MIMO channels. We assume that perfect CSI is available at the receiver and discuss two cases: perfect CSI and no CSI at the transmitter.

\subsubsection{Perfect CSI at Transmitter}

The capacity of the MIMO channel when both transmitter and receiver have perfect CSI is given by $[3,5]$

$$
\bar{C}=\max _{\mathbf{Q}: \operatorname{tr}(\mathbf{Q})=\rho} \log _{2} \operatorname{det}\left(\mathbf{I}+\mathbf{H Q} \mathbf{H}^{H}\right),
$$

where $\mathbf{Q}$ is the input covariance matrix. $\rho$ is the average SNR defined as $\rho=$ $P_{t} / \sigma^{2}$ where $P_{t}$ is the total transmit power and $\sigma^{2}$ is the noise power. In [24], it is shown that the MIMO channel can be decomposed into $\min \left(n_{t}, n_{r}\right)$ parallel independent SISO channels by taking the SVD of the channel matrix $\mathbf{H}$. For the MIMO channel, the SVD of $\mathbf{H}$ is $\mathbf{H}=\mathbf{U D V}^{H}$, where $\mathbf{U} \in \mathbb{C}^{n_{r} \times n_{r}}$ and $\mathbf{V} \in \mathbb{C}^{n_{t} \times n_{t}}$ are unitary matrices and $\mathbf{D}$ is a diagonal matrix containing the $m=$ $\min \left(n_{r}, n_{t}\right)$ singular values of $\mathbf{H}$. Waterfilling is used to allocate the transmit power to these parallel SISO channels. The capacity of the MIMO channel with waterfilling is written as [3]

$$
\tilde{C}_{\mathrm{M}}=\sum_{i=1}^{m} \log _{2}\left(1+\frac{1}{\sigma^{2}}\left(\lambda_{i} \mu-\sigma^{2}\right)^{+}\right),
$$

where $\mu$ is the waterfill level and $\lambda_{i}$ is the $i^{\text {th }}$ eigenvalue of the matrix $\Upsilon \mathbf{\Upsilon}$, given by

$$
\mathbf{\Upsilon}=\left\{\begin{array}{ll}
\mathbf{H H}^{H} & n_{r} \leq n_{t} \\
\mathbf{H}^{H} \mathbf{H} & n_{t} \leq n_{r}
\end{array} .\right.
$$




\subsubsection{No CSI at Transmitter}

When the channel is unknown to the transmitter, but channel statistics are known at the transmitter then it is shown in [3] that the ergodic capacity is maximized by having $\mathbf{Q}=\left(\rho / n_{t}\right) \mathbf{I}$ (i.e., equal power allocation to all the transmit antennas). Thus, the capacity of the MIMO channel can be expressed as

$$
C_{\mathrm{M}}=\log _{2} \operatorname{det}\left(\mathbf{I}+\frac{\rho}{n_{t}} \Upsilon\right)
$$

On the other hand, the SISO capacity is given as

$$
C_{\mathrm{S}}=\log _{2}\left(1+\rho|h|^{2}\right),
$$

where $h$ is the SISO channel coefficient.

\subsection{Summary}

In this chapter we have presented an overview of analytical and standardized MIMO channel models. In analytical MIMO channel models four commonly used channels are discussed: Rayleigh i.i.d., Rician, spatially correlated and temporally correlated channels. In addition to analytical models, MIMO standardized channel models are also discussed. In this thesis, both analytical and standardized channel models are used. We have also discussed the capacity of MIMO channels assuming perfect CSI is available at the receiver. 


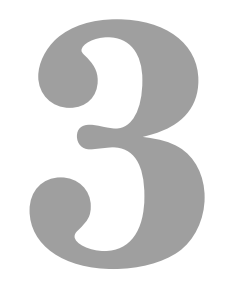

\section{Theoretical Background of Limited Feedback Systems}

In this chapter, we provide a general overview of limited feedback systems. We briefly discuss beamforming and spatial multiplexing for SU systems and linear precoding for MU systems with limited feedback. We explain basic concepts of limited feedback systems, such as codeword selection, quantization errors and feedback interval. We also examine the distribution of the dominant right singular vector for various channels.

\subsection{Limited Feedback Multiple Antenna Systems}

Research in MIMO systems has shown that CSI at the transmitter improves the performance in terms of capacity and reliability. However, providing perfect CSI to the transmitter is not possible under a low-rate feedback control channel from the receiver to the transmitter. Researchers have designed various techniques that try to efficiently utilize this low-rate feedback link, so that the quantized channel information delivered to the transmitter achieves the desired performance with a minimum loss relative to the case where perfect CSI is available. The codebook based limited feedback approach is considered to be a successful technique for this purpose [20]. The block diagram of the basic limited feedback MIMO system is shown in Fig. 3.1.

In limited feedback schemes, the main idea is that the receiver estimates the channel and quantizes it using a codebook. In this thesis, for SU MIMO systems, we quantize the dominant right singular vectors of the channel at the receiver, whereas, in MU systems, we quantize CDI for each user. Generally, the channel 


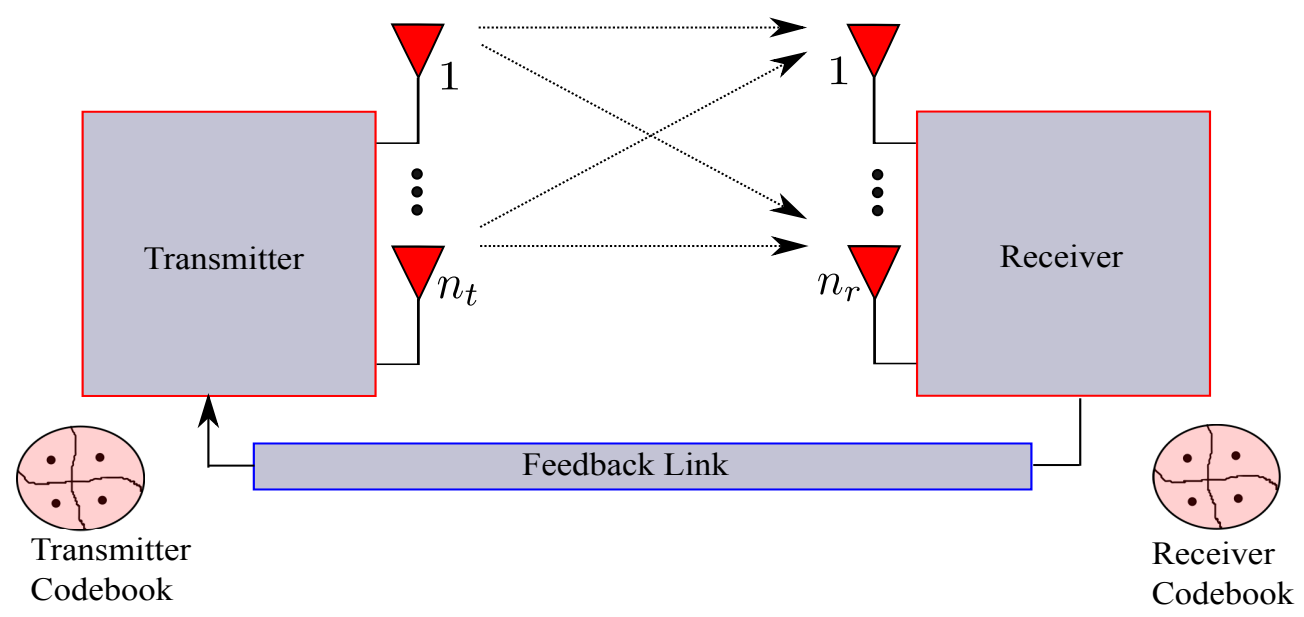

Figure 3.1: The block diagram of a limited feedback MIMO system.

quantization is realized by selecting a codeword that maximizes/minimizes a specific performance/distortion function (SNR, squared chordal distance etc.). Suppose there are $N_{c}$ codewords in the codebook, the receiver selects an appropriate codeword and feeds back the index of the selected codeword to the transmitter via a low-rate feedback link, using $B=\log _{2}\left(N_{c}\right)$ bits. Due to bandwidth limitations, the number of feedback bits required to feedback the index of the selected codeword is considered as feedback overhead. The feedback link is generally assumed to be a lossless with zero delay, for study purposes. There are many studies [63-66] that investigate codebook quantization with delayed and lossy feedback links. At the transmitter, the same codebook is already available and it selects the codebook entry corresponding to the sent index. In SU scenarios, the transmitter may use this codeword directly as a beamforming vector, however, in MU transmission further processing may be required at the BS to eliminate or suppress the interference coming from other users. The main focus of the research in limited feedback systems is to use the minimum number of bits and obtain maximum quality of CSI at the transmitter.

\subsection{SU Systems with Limited Feedback}

In this section, we briefly discuss limited feedback in SU systems. Based on the transmission strategy, SU multiple antenna systems can be categorized into two parts: beamforming and spatial multiplexing.

\subsubsection{Beamforming}

Beamforming achieves array and diversity gains by transmitting the same symbol weighted by a complex number from each transmit antenna. The weights at all the antennas can be collected into a beamforming vector, denoted by $\mathbf{w}$. 
In the case of MIMO, the signals at multiple receive antennas are combined by a weighted summation to obtain the resulting symbol.

It is shown in [8] that the received SNR is maximized when weights at the transmitter and receiver are $\mathbf{v}_{\text {dom }}$ and $\mathbf{u}_{\text {dom }}^{H}$, respectively, where $\mathbf{v}_{\text {dom }}$ and $\mathbf{u}_{\text {dom }}$ are the right and left singular vectors associated with the dominant singular value of the channel, $\mathbf{H}$. This scheme is also known as dominant eigenmode transmission.

In the case of the MISO system, matched-filter (MF) beamforming [67] is used to maximize the receive SNR, where the beamforming vector is given by $\mathbf{w}=\mathbf{h}^{H} /\|\mathbf{h}\|$. This technique is also known as conventional beamforming.

The above discussion is for the case when perfect CSI is available at the transmitter. For limited feedback MIMO/MISO systems, the performance degrades when using quantized beamforming vectors from the codebook. The amount of capacity loss and SNR loss with quantized beamforming is investigated in $[42,68,69]$. The capacities of the MIMO system and the MISO system with limited feedback beamforming vector denoted by $\mathbf{f}$ and $\mathbf{q}$, are given by

$$
C_{\mathrm{MIMO}-\mathrm{BF}}=\log _{2}\left(1+\frac{\rho}{n_{t}}\|\mathbf{H f}\|^{2}\right)
$$

and

$$
C_{\mathrm{MISO}-\mathrm{BF}}=\log _{2}\left(1+\frac{\rho}{n_{t}}|\mathbf{h q}|^{2}\right)
$$

respectively.

There are numerous studies [42,70-72] that examine the performance of MIMO beamforming with limited feedback techniques. Beamforming techniques in limited feedback MISO systems are also well investigated in $[22,27,73]$.

In order to measure the average loss in the array gain due to the quantized beamforming vectors, the distortion function for MIMO systems that is generally used is given by [1]

$$
D_{f}=\mathbb{E}\left[\lambda_{\max }-\|\mathbf{H f}\|^{2}\right]
$$

where $\lambda_{\max }$ is the dominant singular value of the channel $\mathbf{H}$, (array gain with perfect CSI). The quantized codebook beamforming vector is denoted f. Similarly for the MISO system, the distortion function is given by

$$
D_{f}=\mathbb{E}\left[1-|\mathbf{h q}|^{2}\right]
$$

where $\mathbf{q}$ is the selected beamforming vector from the codebook. The distortion function for various codebook designs is thoroughly discussed in [1]. 


\subsubsection{Spatial Multiplexing}

A MIMO channel can be decomposed into multiple parallel independent channels depending on the rank of the channel. MIMO systems provide multiplexing gain by transmitting independent data symbols through these independent channels. Such systems are also known as MIMO spatial multiplexing systems. The multiplexing gain increases the data rate of the system [3].

Given that $\operatorname{SVD}(\mathbf{H})=\mathbf{U D V}^{H}$, the parallel decomposition of the MIMO channel is realized by transforming the input data vector and received data vector by $\mathbf{V}$ and $\mathbf{U}^{H}$, respectively. The transformation at the transmitter is known as transmit precoding and the transformation at the receiver is called receiver shaping [23].

A limited feedback MIMO system with spatial multiplexing is studied in [74], where optimal precoding matrix selection criteria is presented for various MIMO receivers. It is also shown in [74] that the codebook design criteria for all the receivers are related and equivalent to subspace packing in a Grassmannian manifold. Denoting the limited feedback MIMO precoding matrix by $\mathbf{F}$, the capacity of the MIMO spatial multiplexing system is given by

$$
C_{\mathrm{MIMO}-\mathrm{SM}}=\log _{2} \operatorname{det}\left(\mathbf{I}+\frac{\rho}{n_{t}} \mathbf{F}^{H} \mathbf{H}^{H} \mathbf{H F}\right) \text {. }
$$

In multimode MIMO systems, unlike single-mode, the number of transmission streams are not fixed but vary according to the channel state [1,75]. For limited feedback multimode MIMO systems, the adaptive transmission is studied in [75], where adaptation is performed by varying the number of streams in order to minimize the bit error rate for the given data rate.

\subsection{MU Systems with Limited Feedback}

In this section, we restrict the limited feedback discussion to MISO antenna configurations only, as in this thesis, we use MU MISO systems. In a MU MISO system, the BS with $n_{t}$ antennas serves $K$ single antenna receivers (users), simultaneously in the same time and frequency resource. Each user quantizes its own channel (CDI) and use the limited feedback link to send the index of the appropriate codeword to the BS. The BS compute the precoding vectors for each user using the quantized channel information. The sum-rate of the MU MISO system is [27]

$$
C_{\text {sum }}=\sum_{k=1}^{K} \log _{2}\left(1+\mathrm{SINR}_{k}\right)
$$


where the SINR of the $k^{\text {th }}$ user is given by

$$
\mathrm{SINR}_{k}=\frac{\frac{\rho}{K}\left|\mathbf{h}_{k} \mathbf{w}_{k}\right|^{2}}{1+\frac{\rho}{K} \sum_{j \neq k}^{K}\left|\mathbf{h}_{k} \mathbf{w}_{j}\right|^{2}}
$$

where $\mathbf{h}_{k}$ and $\mathbf{w}_{k}$ denote the channel and the precoding vector for the $k^{\text {th }}$ user.

It is assumed throughout the thesis that the transmitter has perfect knowledge of the channel quality indicator (CQI) for each receiver, where CQI $=\left\|\mathbf{h}_{k}\right\|$. The selected codeword from the codebook at the $k^{\text {th }}$ user is denoted by $\mathbf{q}_{k}$, which represents the quantized version of the perfect CDI given by $\mathbf{u}_{k}=\mathbf{h}_{k} /\left\|\mathbf{h}_{k}\right\|$. The BS constructs the quantized channel for the $k^{\text {th }}$ user, $\mathbf{g}_{k}$, using and the CQI and the quantized CDI, $\mathbf{q}_{k}$, such that $\mathbf{g}_{k}=\left\|\mathbf{h}_{k}\right\| \mathbf{q}_{k}{ }^{T}$.

The precoding schemes used in this thesis are ZF, RZF, MF and signalto-leakage-plus-noise ratio (SLNR). An overview of these precoding schemes is given in the following subsections.

\subsubsection{ZF Precoding}

ZF precoding $[25,76]$ is one of the most commonly used precoding strategies in MU MISO systems. With perfect CSI, the ZF precoding vectors are formed such that for the $k^{\text {th }}$ user, the product $\mathbf{h}_{k} \mathbf{w}_{j}$ is zero, for all $k \neq j$, where $\mathbf{h}_{k}$ and $\mathbf{w}_{j}$ are the $k^{\text {th }}$ user channel and the precoding vector of the $j^{\text {th }}$ user, respectively. In the case of a limited feedback system, ZF precoding cannot cancel the MU interference completely but is still able to reduce it significantly [27]. For a total of $K$ number of users in the system, the quantized $K \times n_{t}$ channel matrix, $\mathbf{H}_{\mathrm{zf}}$, is constructed at the BS, given by $\mathbf{H}_{\mathrm{zf}}=\left[\begin{array}{llll}\mathbf{g}_{1}^{T} & \cdots & \mathbf{g}_{K}^{T}\end{array}\right]^{T}$, where $\mathbf{g}_{1}$ is the quantized channel of the $1^{\text {st }}$ user. A beamforming weight matrix is obtained by taking the right pseudo-inverse of $\mathbf{H}_{\mathrm{zf}}$ given by $\mathbf{W}_{\mathrm{zf}}=\mathbf{H}_{\mathrm{zf}}^{H}\left(\mathbf{H}_{\mathrm{zf}} \mathbf{H}_{\mathrm{zf}}^{H}\right)^{-1}$. The $\mathrm{ZF}$ precoding vector for the $k^{\text {th }}$ user, $\mathbf{w}_{k}$, is the normalized $k^{\text {th }}$ column of $\mathbf{W}$. In $\mathrm{ZF}$ precoding, there is a constraint that the total number of users cannot be greater that the number of transmit antennas, i.e., $K \leq n_{t}$.

\subsubsection{RZF Precoding}

RZF precoding is introduced in [77]. It performs better than ZF precoding in the low SNR regime while matching ZF precoding performance at high SNR. In RZF precoding, a regularization parameter, $\zeta$, is designed to maximize or minimize a certain performance metric such as SINR. In [77], $\zeta$ is designed for homogeneous users (users having same mean link gain), that maximizes the SINR, given by $\zeta=K / \rho$. The RZF precoding matrix is written as

$$
\mathbf{W}_{\mathrm{rzf}}=\mathbf{H}_{\mathrm{rzf}}^{H}\left(\mathbf{H}_{\mathrm{rzf}} \mathbf{H}_{\mathrm{rzf}}^{H}+\zeta \mathbf{I}\right)^{-1}
$$


where $\mathbf{H}_{\mathrm{rzf}}=\left[\begin{array}{lll}\mathbf{g}_{1}^{T} & \cdots & \mathbf{g}_{K}^{T}\end{array}\right]^{T}$. The RZF precoding vector for the $k^{\text {th }}$ user, $\mathbf{w}_{k}$, is the normalized $k^{\text {th }}$ column of $\mathbf{W}$. By selecting $\zeta=0$, RZF precoding becomes equivalent to $\mathrm{ZF}$ precoding.

\subsubsection{MF Precoding}

MF precoding is the simplest form of precoding used in MU MISO systems. The precoding vector is a conjugate of the CDI, such that for the $k^{\text {th }}$ user, $\mathbf{w}_{k}=\mathbf{u}_{k}^{H}$ (for perfect CDI) and $\mathbf{w}_{k}=\mathbf{q}_{k}^{H}$ (for codebook CDI). The MF precoding maximizes the received SNR at each user [1], but it totally ignores the interference, especially when the interfering channels lie close to the desired channel in a vector space.

\subsubsection{SLNR Precoding}

The main idea of SLNR precoding is to minimize the total power leaked from a user to all other users, while maintaining a strong SNR. The SLNR precoding solution is provided in [78] for the perfect CSI case. In limited feedback systems, the leakage from the user $k$ is approximated by $\sum_{i=1, k \neq i}^{K}\left|\mathbf{g}_{i} \mathbf{w}_{k}\right|^{2}$. The SLNR precoding vector, $\mathbf{w}_{k}$, for the $k^{\text {th }}$ user is the normalized maximum eigenvector of $\left(K / \rho_{k} \mathbf{I}+\mathbf{H}_{\mathrm{slnr}, k}^{H} \mathbf{H}_{\mathrm{slnr}, k}\right)^{-1} \mathbf{g}_{k}^{H} \mathbf{g}_{k}$, where $\mathbf{H}_{\mathrm{sln}, k}$ is a quantized channel matrix of size $(K-1) \times n_{t}$ for the $k^{\text {th }}$ user constructed from the codebook entries fed back by the other users to the transmitter, such that, $\mathbf{H}_{\mathrm{s} \ln , k}=\left[\mathbf{g}_{1}^{T} \cdots \mathbf{g}_{k-1}^{T} \overline{\mathbf{g}}_{k+1}^{T} \cdots \mathbf{g}_{K}^{T}\right]^{T}$. The SNR of the $k^{\text {th }}$ user is denoted by $\rho_{k}$. Figure 3.2 shows SLNR precoding for the limited feedback MU MISO system with leakage from the user 1.

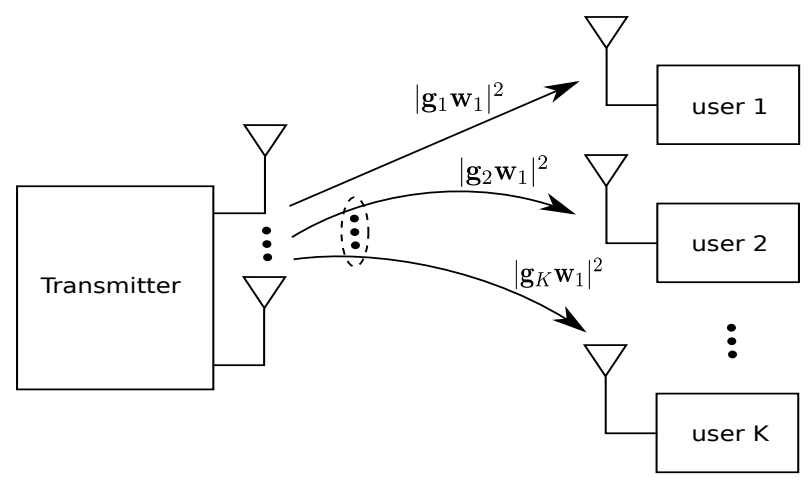

Figure 3.2: The block diagram of SLNR precoding for the limited feedback MU MISO system.

In the following sections, we briefly explain some important concepts in limited feedback theory: codeword selection, quantization errors and feedback interval. 


\subsection{Codeword Selection}

The codeword selection is an important step in a codebook-based limited feedback system, where the receiver selects an appropriate codeword from the codebook that minimizes/maximizes a given distortion/performance metric.

In SU single-stream MIMO transmission, the dominant right singular vector, $\mathbf{v}_{\text {dom }}$, is selected as the optimal beamforming choice to maximize SNR [1] and to achieve array and diversity gains. On the other hand, in SU multi-stream MIMO transmission, multiple dominant right singular vectors, $\mathbf{V}_{\text {dom }}$, are selected as the precoding matrix to achieve multiplexing gains.

Denoting $L$ as the number of transmission streams, consider a codebook having $N_{c}=2^{B}$ codewords representing the quantized dominant $L$ columns of $\mathbf{V}$. The codebook at the transmitter and the receiver is given by, $\mathcal{F}_{\mathrm{M}-\mathrm{S}}=$ $\left[\mathbf{F}^{(1)}, \mathbf{F}^{(2)}, \ldots, \mathbf{F}^{\left(2^{B}\right)}\right]$, for multi-stream transmission. Thus, for $L \leq m$ transmitted data streams, the size of each codeword in the codebook is $n_{t} \times L$.

In single-stream MIMO transmission, the codebook is denoted by $\mathcal{F}_{\mathrm{S}-\mathrm{S}}=$ $\left[\mathbf{f}^{(1)}, \mathbf{f}^{(2)}, \ldots, \mathbf{f}^{\left(2^{B}\right)}\right]$, where each codeword is a column vector of size $n_{t} \times 1$.

In the MISO channel, channel direction information (CDI) given by $\mathbf{u}=$ $\mathbf{h} /\|\mathbf{h}\|$ is quantized at the receiver using the single-stream codebook, $\mathcal{F}_{\mathrm{S}-\mathrm{S}}$.

There are many distortion/performance based metrics developed in the literature to select an appropriate codeword from the codebook. Some of them are briefly discussed in the following subsections.

\subsubsection{SNR Maximization}

In this method, for MIMO multi-stream transmission, the receiver selects a codeword that maximizes the SNR, such that [1]

$$
\mathbf{F}=\arg \max _{\substack{\mathbf{F}^{(i)} \in \mathcal{F}_{\mathrm{M}-\mathrm{S}} \\ 1 \leq i \leq 2^{B}}}\left\|\mathbf{H F}^{(i)}\right\|_{F}^{2} .
$$

Similarly, for the single-stream MIMO, we can express SNR maximization selection criteria as

$$
\mathbf{f}=\arg \max _{\substack{\mathbf{f}^{(i)} \in \mathcal{F}_{\mathrm{S} S} \\ 1 \leq i \leq 2^{B}}}\left\|\mathbf{H f}^{(i)}\right\|^{2} .
$$

For a MISO system, the codeword selection that maximizes the SNR is given by

$$
\mathbf{q}=\arg \max _{\substack{\mathbf{f}^{(i)} \in \mathcal{F}_{\mathrm{S}_{-}} \\ 1 \leq i \leq 2^{B}}}\left|\mathbf{h f}^{(i)}\right|^{2} .
$$

The SNR maximization codeword selection method is one of the most common type of selection criteria in MIMO/MISO limited feedback systems. 


\subsubsection{Squared Chordal Distance}

Chordal distance [79] is a measure of distance between two matrices or vectors. Like SNR maximization method, the squared chordal distance based codeword selection criteria is also commonly used. For the MIMO system with multistream transmission, the codeword selection according to the minimum squared chordal distance is given by

$$
\mathbf{F}=\arg \min _{\substack{\mathbf{F}^{(i)} \in \mathcal{F}_{\mathrm{M}-\mathrm{S}} \\ 1 \leq i \leq 2^{B}}} L-\sum_{j=1}^{L}\left|\mathbf{v}_{j}^{H} \mathbf{f}_{j}^{(i)}\right|^{2}
$$

where $\mathbf{v}_{j}$ and $\mathbf{f}_{j}^{(i)}$ are the $j^{\text {th }}$ columns of the right singular matrix $\mathbf{V}_{\text {dom }}$ and the $i^{\text {th }}$ codebook entry, $\mathbf{F}^{(i)}$, respectively. For the single-stream MIMO system, the minimum squared chordal distance based codeword selection criteria is given by

$$
\mathbf{f}=\arg \min _{\substack{\mathbf{f}^{(i)} \in \mathcal{F}_{\mathrm{S} S} \\ 1 \leq i \leq 2^{B}}} 1-\left|\mathbf{v}_{\mathrm{dom}}^{H} \mathbf{f}^{(i)}\right|^{2} .
$$

For the MISO channel, the selected codeword, determined by the minimum squared chordal distance, is written as

$$
\mathbf{q}=\arg \min _{\substack{\mathbf{f}^{(i)} \in \mathcal{F}_{\mathrm{S}-\mathrm{S}} \\ 1 \leq i \leq 2^{B}}} 1-\left|\mathbf{u f}^{(i)}\right|^{2}
$$

\subsubsection{Capacity Maximization}

The capacity maximization [74] based selection method selects a codeword that maximizes the capacity of the SU MIMO system. The selection criteria is given by

$$
\mathbf{F}=\arg \max _{\substack{\mathbf{F}^{(i)} \in \mathcal{F}_{\mathrm{M}-\mathrm{S}} \\ 1 \leq i \leq 2^{B}}} \log _{2} \operatorname{det}\left(\mathbf{I}+\frac{\rho}{n_{t}} \mathbf{F}^{(i) H} \mathbf{H}^{H} \mathbf{H} \mathbf{F}^{(i)}\right),
$$

where $\rho$ is the SNR of the channel. In the case of MIMO single-stream transmission, the capacity or mutual information maximization based codeword selection is performed by [70]

$$
\mathbf{f}=\arg \max _{\substack{\mathbf{f}^{(i)} \in \mathcal{F}_{\mathrm{S}-\mathrm{S}} \\ 1 \leq i \leq 2^{B}}} \log _{2}\left(1+\frac{\rho}{n_{t}}\left\|\mathbf{H f}^{(i)}\right\|^{2}\right) .
$$

Similarly for a MISO system, capacity maximization selection is given by [73]

$$
\mathbf{q}=\arg \max _{\substack{\mathbf{f}^{(i)} \in \mathcal{F}_{\mathrm{S}_{-} \mathrm{S}} \\ 1 \leq i \leq 2^{B}}} \log _{2}\left(1+\frac{\rho}{n_{t}}\left|\mathbf{h} \mathbf{f}^{(i)}\right|^{2}\right) .
$$




\subsubsection{Random Selection}

In a random codeword selection [68], the codeword is selected randomly from the codebook without maximizing/minimizing any performance/distortion metric. Although, this selection scheme may not select a worst codeword from the codebook, but it is often used for comparison purposes [68].

In Chapter 4, we use squared chordal distance as the selection criteria for SU MIMO systems. However, for MU MISO systems, we use SNR maximization based codeword selection criteria.

\subsection{Codebook Quantization Errors}

In a limited feedback MISO system, the mismatch between the selected codeword, $\mathbf{q}$, and the perfect CDI, u, results in codebook quantization errors (we remove the user subscript $k$ for simplicity). Quantization errors degrade the performance of MIMO/MISO systems. In MISO systems, quantization error is expressed as

$$
\begin{aligned}
\mathcal{Q} & =\sin ^{2}\left(\angle\left(\mathbf{u}^{H}, \mathbf{q}\right)\right) \\
& =1-|\mathbf{u q}|^{2} .
\end{aligned}
$$

For RVQ codebooks, the mean quantization error in i.i.d. Rayleigh channels has been shown to be upper bounded by [22]

$$
\begin{aligned}
\mathbb{E}[\mathcal{Q}] & =2^{B} \beta\left(2^{B}, \frac{n_{t}}{n_{t}-1}\right) \\
& \leq 2^{-\frac{B}{n_{t}-1}},
\end{aligned}
$$

where $B$ is the number of feedback bits and $\beta(\cdot, \cdot)$ represents the beta function. It can be noticed that the larger the number of feedback bits, the smaller the mean quantization error becomes. However, it is not practically acceptable to increase $B$, and it is desirable to have minimum quantization errors with a small number of feedback bits. The mean quantization errors for the codebooks such as DFT and channel distribution information at the transmitter (CDIT) are discussed in [1] for spatially correlated MISO channels.

For a MIMO system, the total quantization error can be written as the sum 
of quantization errors of each stream;

$$
\begin{aligned}
\mathcal{Q} & =\sum_{j=1}^{L} \sin ^{2}\left(\angle\left(\mathbf{v}_{j}, \mathbf{f}_{j}\right)\right) \\
& =\sum_{j=1}^{L} 1-\left|\mathbf{v}_{j}^{H} \mathbf{f}_{j}\right|^{2}
\end{aligned}
$$

where $\mathbf{v}_{j}$ and $\mathbf{f}_{j}$ are the $j^{\text {th }}$ columns of the right singular matrix $\mathbf{V}_{\text {dom }}$ and the selected codebook entry, $\mathbf{F}$, respectively.

\subsection{Feedback Update Interval}

In limited feedback systems, sufficiently frequent CSI feedback is required from the receiver to the transmitter for beamforming or precoding purposes. Specifically, it is necessary to feedback the CSI within a time which is less than the coherence time, $T_{c}$, of the channel, so that the channel between the transmitter and the receiver is not changed significantly. For reasonable performance, it is important to feedback the CSI to the transmitter well before the channel coherence time, such that, $T_{\mathrm{fb}} \ll T_{c}$, where $T_{\mathrm{fb}}$ is the feedback update interval.

Although, the coherence time of the channel can be measured but there is no standard calculation of the feedback interval for fast fading channels. For slow fading channels with temporal correlation, there are a few studies [68,80-82] that deal with the feedback update period.

To evaluate the performance of limited feedback systems in temporally correlated channels, wireless standards contributions and most of the previous work $[46,48,83]$ use 5 milliseconds (ms) as the feedback update interval. Therefore, in this thesis we also set the feedback update interval to be $5 \mathrm{~ms}$ in slow fading channels.

\subsection{MIMO Channels and Codebooks}

Early limited feedback studies considered Rayleigh i.i.d. channels and later the focus of the research shifted towards more practical spatially and temporally correlated channels. It is important to design a codebook that takes into account the effects of the propagation environment, as different propagation environments may need different codebooks. In Chapter 2, we discussed analytical MIMO channels: Rayleigh i.i.d., Rician, spatially correlated and temporally correlated MIMO channels.

The distribution of the right singular vectors (corresponding to the dominant singular value of the channel) on a unit sphere for $2 \times 2$ MIMO channels, is 


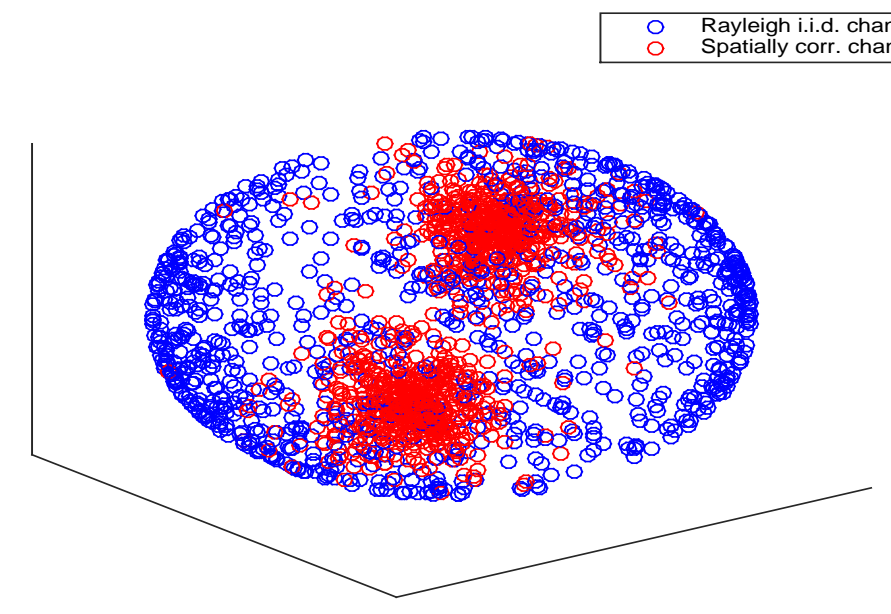

Figure 3.3: The distribution of right singular vectors on the unit sphere for the Rayleigh i.i.d. and spatially correlated $2 \times 2$ MIMO channels.

illustrated in Fig. 3.3 for the Rayleigh i.i.d. and spatially correlated channels. In Fig. 3.3, it is seen that the right singular vectors of the channel are uniformly distributed across the entire unit sphere in the case of Rayleigh i.i.d. channel, however, they form clusters for spatially correlated channels with $z_{\mathrm{t}}=z_{\mathrm{r}}=0.95$ in (2.9) and (2.10). The figure helps to understand what sort of codebooks are required to deal with such channels. For example, in Rayleigh i.i.d. channels (blue circles) in Fig. 3.3, it is evident that in order to effectively quantize the channel, a codebook structure should have codewords that are uniformly distributed on the sphere. For spatially correlated channels (red circles), in Fig. 3.3, the effective codebook should have a cap like structure with clustered

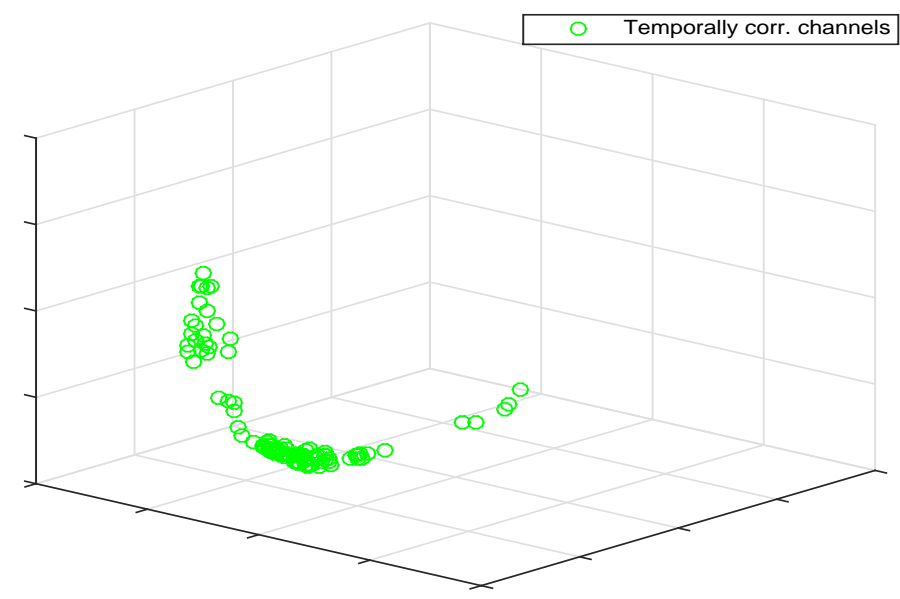

Figure 3.4: The distribution of right singular vectors on the unit sphere for the temporally correlated Rayleigh $2 \times 2$ MIMO channel with 100 time samples. 


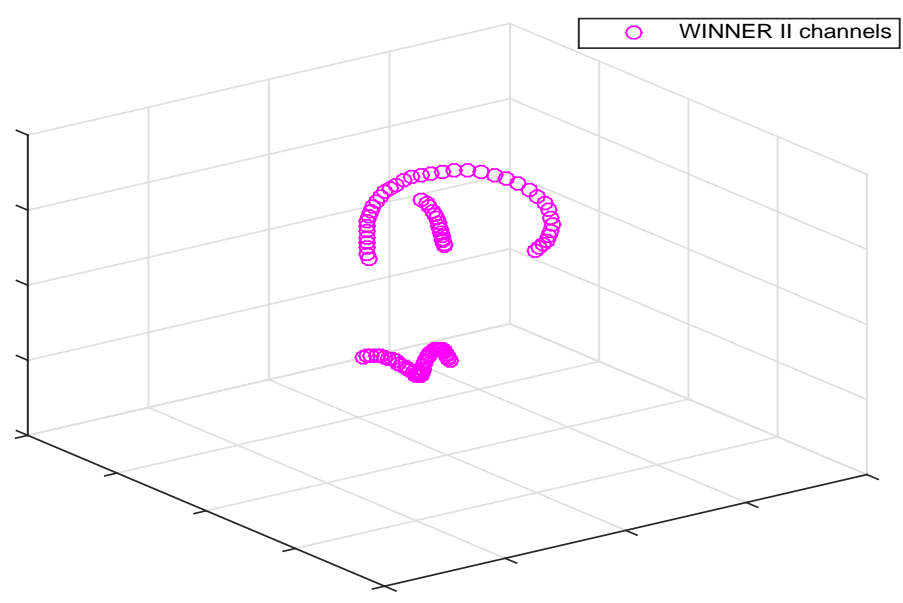

Figure 3.5: The distribution of right singular vectors on the unit sphere for the WINNER II $2 \times 2$ MIMO channel with 100 time samples.

codewords.

The right singular vectors for the temporally correlated channel are plotted in Fig. 3.4 for 100 consecutive time intervals with $\epsilon=0.9987$ for carrier frequency $2.5 \mathrm{GHz}$ and velocity, $v=1 \mathrm{~km} / \mathrm{h}$, in (2.12). The vectors generally lie very close to previous right singular vectors and follow a trajectory on the unit sphere as seen in Fig. 3.4. In Fig. 3.4, the channel lies close to the previous channel in time, therefore in order to successfully track the slowly varying channel, a codebook should be able to transform itself after each feedback interval. Similarly, it is noted in Fig. 3.5 for the WINNER II channel, with velocity $v=1$ $\mathrm{km} / \mathrm{h}$ and $0.5 \lambda_{0}$ antenna spacing, that the right singular vectors lie close to each other and similar to Fig. 3.4, the entries also follow a trajectory. The main goal of an effective codebook design is to reduce quantization errors by taking into account factors such as propagation environment, antenna polarizations and channel correlation statistics.

\subsection{Summary}

In this chapter, we briefly discussed important concepts of limited feedback MIMO/MISO systems. For SU systems, we discussed beamforming and spatial multiplexing. We have provided an overview of various precoding schemes for limited feedback MU MISO systems as used in this thesis. 


\section{4 \\ MIMO Capacity Analysis}

In this chapter, the capacity gains of the MIMO system over the SISO system with full and no CSI at the transmitter are discussed. We then consider a limited feedback SU MIMO system and derive capacity loss expressions for various linear receivers; the capacity loss is defined as the difference between the capacity with perfect CSI feedback and the capacity with codebook feedback. The capacity loss analysis considers both analytical and standardized MIMO channel models discussed in the previous chapter. The capacity loss study is focused on singlestream and two-stream MIMO transmission modes. The results in this chapter serves as a motivation for various codebook designs in the following chapters.

\subsection{MIMO Capacity Gains}

In [84], we investigate the MIMO capacity gains ${ }^{1}$ relative to the SISO capacity using two metrics: the ratio between the expected value of the two capacities and the difference between the two capacities. We derive limiting values of the two capacity metrics for low and high SNR regimes and show via simulation their behavior at other values of SNR. We also examine a range of correlation conditions using the spatially correlated Kronecker channel model described in Chapter 2 Section 2.2.3. In [84], we do not consider a limited feedback system and assume either perfect CSI or no CSI is available at the transmitter.

The difference, $D$, between the expected MIMO capacity and the expected

\footnotetext{
${ }^{1}$ The analysis of MIMO capacity gain has been adopted from [84], this analysis serves as a motivation to investigate MIMO channels.
} 
SISO capacity is defined as

$$
D=\mathbb{E}\left[\mathrm{C}_{\mathrm{M}}\right]-m \mathbb{E}\left[\mathrm{C}_{\mathrm{S}}\right]
$$

where $\mathrm{C}_{\mathrm{M}}$ and $\mathrm{C}_{\mathrm{S}}$ are the capacities of MIMO and SISO systems given in (2.22) and (2.23), respectively. $m$ is given by $m=\min \left(n_{t}, n_{r}\right)$. Similarly, the ratio of the MIMO capacity to the SISO capacity, denoted by $R$ is defined as

$$
R=\frac{\mathbb{E}\left[\mathrm{C}_{\mathrm{M}}\right]}{\mathbb{E}\left[\mathrm{C}_{\mathrm{S}}\right]}
$$

The results derived in [84] are summarized in Table 4.1, for limiting values of SNR, $\rho=P_{t} / \sigma^{2}$, under the i.i.d. Rayleigh channel and the fully correlated Kronecker channel (2.8), such that $z_{t}=z_{r}=1$. Here $\tilde{D}$ and $\tilde{R}$ represent the capacity difference and capacity ratio when full CSI is available at the transmitter, given by

$$
\tilde{D}=\mathbb{E}\left[\tilde{\mathrm{C}}_{\mathrm{M}}\right]-m \mathbb{E}\left[\mathrm{C}_{\mathrm{S}}\right]
$$

and

$$
\tilde{R}=\frac{\mathbb{E}\left[\tilde{\mathrm{C}}_{\mathrm{M}}\right]}{\mathbb{E}\left[\mathrm{C}_{\mathrm{S}}\right]},
$$

respectively, where $\tilde{\mathrm{C}}_{\mathrm{M}}$ is given by $(2.20)$. In Table $4.1, D_{\infty}$ is a constant that could be positive or negative depending on the channel statistics and is shown

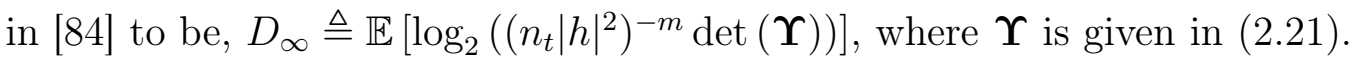

Table 4.1: Capacity gain results: ratio, $R$, and difference, $D$ [84].

\begin{tabular}{|c|c|c|c|c|c|}
\hline \multicolumn{2}{|c|}{} & \multicolumn{2}{|c|}{ i.i.d. Rayleigh Channel } & \multicolumn{2}{c|}{ Fully Correlated Channel } \\
\cline { 3 - 6 } \multicolumn{2}{|c|}{} & $\rho \rightarrow 0$ & $\rho \rightarrow \infty$ & $\rho \rightarrow 0$ & $\rho \rightarrow \infty$ \\
\hline \multirow{2}{*}{ No CSI } & $R$ & $n_{r}$ & $m$ & $n_{r}$ & 1 \\
\cline { 2 - 6 } & $D$ & 0 & $D_{\infty}$ & 0 & $-\infty$ \\
\hline \multirow{2}{*}{ Full CSI } & $\tilde{R}$ & $\mathbb{E}\left(\lambda_{1}\right)$ & $m$ & $n_{t} n_{r}$ & 1 \\
\cline { 2 - 6 } & $\tilde{D}$ & 0 & $D_{\infty}+m \log _{2} \frac{n_{t}}{m}$ & 0 & $\log _{2} n_{t} n_{r}(m=1),-\infty(m>1)$ \\
\hline
\end{tabular}

Figures 4.1 and 4.2 show the capacity difference, $D$, and ratio, $R$, for i.i.d. Rayleigh and spatially correlated channels when each eigenchannel is given equal power. The antenna configurations are denoted as $n_{t} \times n_{r}$ in the figures. The results with i.i.d Rayleigh channels agree with the limits given in Table 4.1. Troughs in $R$ for $2 \times 2$ and $4 \times 4$ systems are visible in Fig. 4.2 indicating an intermediate drop in the value of $R$ before returning to the limit. A peak in $R$ is observed for a $4 \times 2$ system, in the -5 to $20 \mathrm{~dB}$ SNR range. The capacity scaling of $m$ in MIMO relative to SISO is not achieved for $n_{t}=n_{r}$ systems and it is seen that correlation also reduces the gain. As shown in Fig. 4.2, there are 


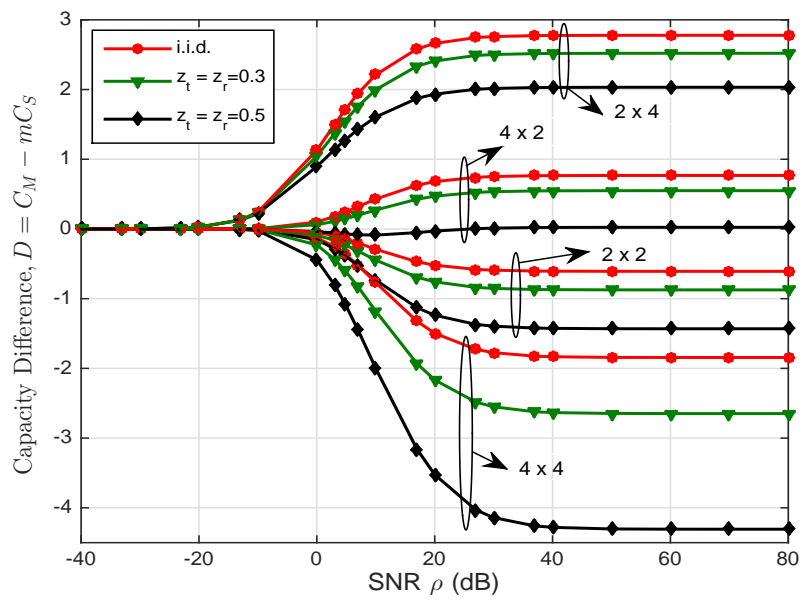

Figure 4.1: $D$ with equal power and spatial correlation at both transmitter and receiver.

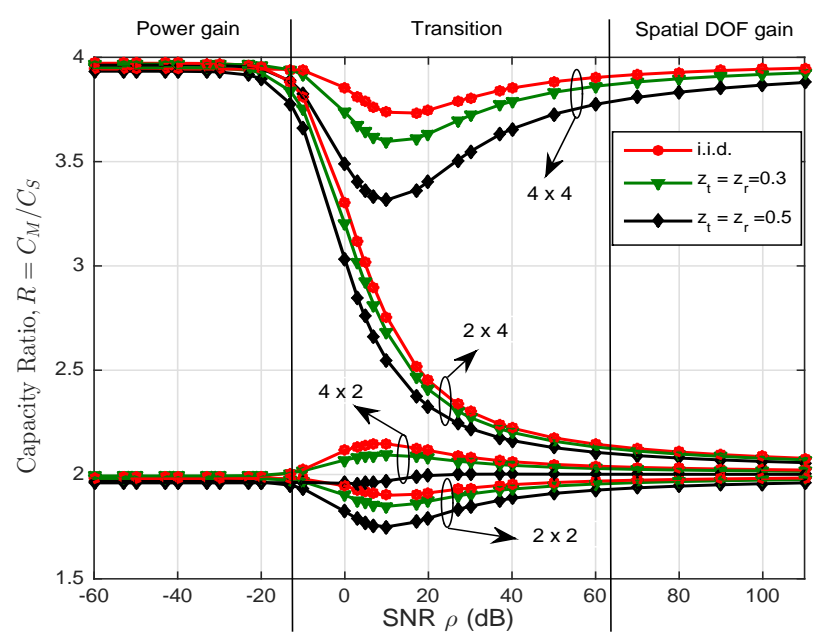

Figure 4.2: $R$ with equal power and spatial correlation at both transmitter and receiver.

two kinds of gain; the power gain regime at low SNR and the spatial degrees of freedom (DOF) gain at high SNR.

Figures 4.3 and 4.4 show the capacity difference and ratio, respectively, with waterfilling power allocation for i.i.d. Rayleigh and spatially correlated channels. We note that the difference, $\tilde{D}$, in Fig. 4.3 with waterfilling is higher for $n_{t} \neq n_{r}$ systems compared to $n_{t}=n_{r}$ systems, at high SNR values. On the other hand, the ratio, $\tilde{R}$, in Fig. 4.4 with waterfilling yields higher gains at low SNR values compared to the $R$ (in Fig. 4.2), but eventually drops down to $R$, for high SNR values. 


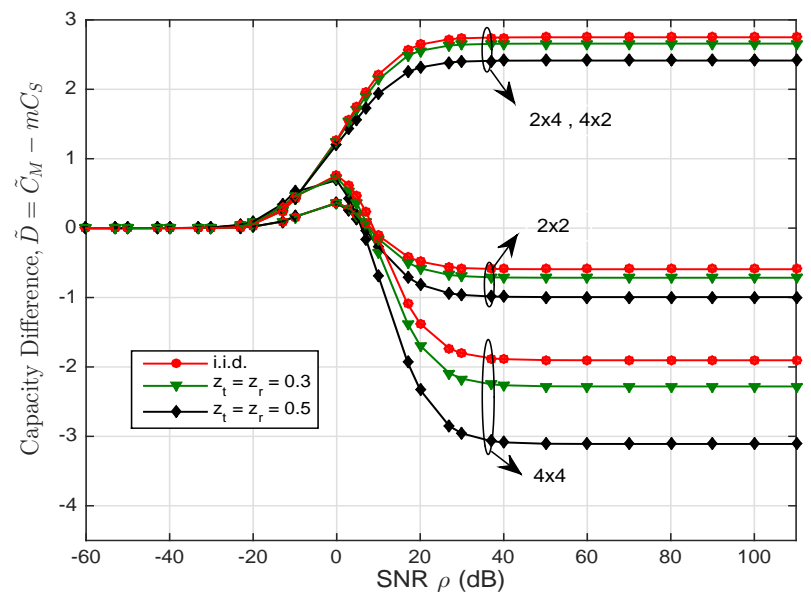

Figure 4.3: $\tilde{D}$ with waterfilling and spatial correlation at both transmitter and receiver.

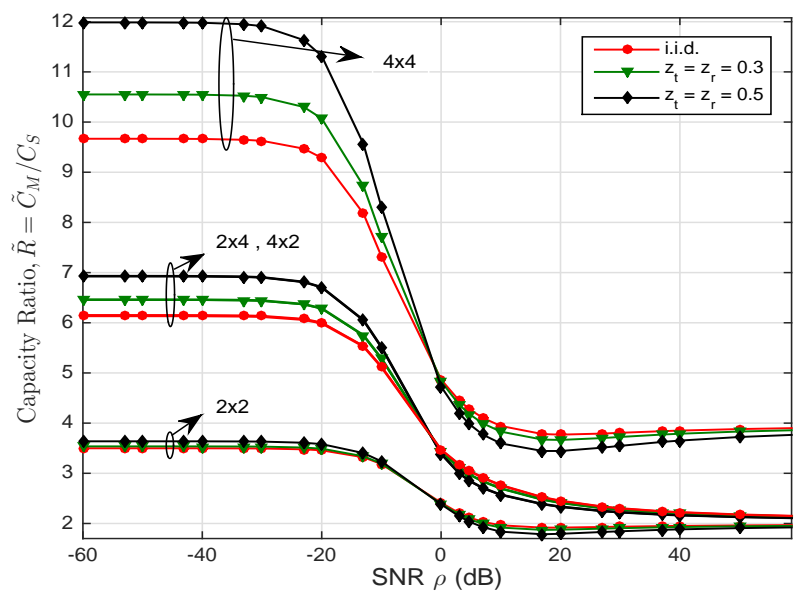

Figure 4.4: $\tilde{R}$ with waterfilling and spatial correlation at both transmitter and receiver.

\subsection{MIMO Capacity Loss Analysis with Limited Feedback}

In this section, we present capacity loss analysis with limited feedback systems and various MIMO receivers. There are many studies that deal with estimating and limiting the capacity loss. In [85], a codebook design for a multimode MIMO system is proposed that bounds the capacity loss. The effects of limited feedback on the performance of MIMO systems over an i.i.d. Rayleigh flat fading channel are studied in [86] and tight lower bounds are derived for the capacity loss due to limited feedback for the MIMO systems employing a fixed number of equal power spatial streams (layers). A similar study is also done in [87]. 
A bound on the ergodic capacity loss for a limited feedback MIMO system is given in [88] when random codebooks, generated from the uniform distribution on the complex unit sphere are used. The authors in [88] provide a closed-form expression for the ergodic capacity loss as a function of the number of feedback bits.

With limited numbers of codebook entries, it is rarely possible to find a codebook entry that has a zero or negligible distance from the corresponding channel values. A non zero distance (or quantization error) manifests itself as a capacity loss arising mainly due to interstream interference.

While there are a number of studies that determine or bound the capacity loss, or determine the number of feedback bits required to minimize the capacity loss, the bulk of these papers consider an i.i.d. flat fading Rayleigh channel $[27,85-88]$. Yet in practical deployments there is always some amount of spatial and temporal correlation present. We examine the capacity loss for codebooks that are already standardized and used in practical settings.

Some useful capacity loss expressions are derived for MIMO systems in [68], that explain the impact of capacity loss on codebook design, selection criteria and receiver design. Motivated by [68], we evaluate and quantify the amount of capacity loss in [69] with LTE and Grassmannian codebooks with singlestream/Layer 1 (L1) and two-stream/Layer 2 (L2) transmission modes in three different channel models. We derive capacity loss approximations for ZF, minimum mean-squared error (MMSE) and SVD receivers in terms of the minimum squared chordal distance [69]. We find that the capacity loss approximations for single-stream transmission are accurate for all receivers and lead to an extremely simple relationship between capacity loss and codebook quantization errors. We evaluate the distribution of the quantization errors for LTE and Grassmannian codebooks via simulations.

\subsubsection{System Description}

We consider a SU limited feedback MIMO-OFDM system with $n_{t}$ transmit and $n_{r}$ receive antennas, where, for a single subcarrier, the frequency flat fading channel is denoted by $\mathbf{H}$.

In the case of multi-stream transmission, the transmitter encodes the data sequence, s, by a precoding matrix, F, selected from a codebook of quantized values of the first $L$ columns of right singular matrix $\mathbf{V}$, denoted by $\mathcal{F}_{\mathrm{M}-\mathrm{S}}=\left[\mathbf{F}^{(1)}, \mathbf{F}^{(2)}, \ldots, \mathbf{F}^{\left(2^{B}\right)}\right]$, where $L$ is the total number of transmission streams. Thus, for $L \leq m$ transmitted data streams, the codebook consists of $2^{B}$ codewords each of size $n_{t} \times L$. For the single-stream transmission, the precoding vector, $\mathbf{f}$, is selected from the codebook, $\mathcal{F}_{\mathrm{S}-\mathrm{S}}=\left[\mathbf{f}^{(1)}, \mathbf{f}^{(2)}, \ldots, \mathbf{f}^{\left(2^{B}\right)}\right]$.

The codeword selection for multi-stream and single-stream is performed ac- 
cording to the minimum squared chordal distance given in (3.12) and (3.13), respectively. Denoting by $\mathbf{v}_{k}$ and $\mathbf{f}_{k}$, the $k^{\text {th }}$ column of $\mathbf{V}$ and $\mathbf{F}$, respectively, the minimum squared chordal distance, $d_{\min _{k}}$, is given by

$$
d_{\min _{k}}=1-\left|\mathbf{v}_{k}^{H} \mathbf{f}_{k}\right|^{2}
$$

The index of the selected codeword is fed back to the transmitter and the resulting received signal for multi-stream and single-stream is given by

$$
\mathbf{y}=\sqrt{\rho} \mathbf{H F s}+\mathbf{n},
$$

and

$$
\mathbf{y}=\sqrt{\rho} \mathbf{H f s}+\mathbf{n},
$$

respectively, where $\mathbf{n}$ is a vector of i.i.d. complex Gaussian $\mathcal{C N}\left(0, \sigma^{2}\right)$ random variables. We have $\mathbb{E}\left[\mathbf{s s}^{H}\right]=(1 / L) \mathbf{I}$ and $\operatorname{tr}\left(\mathbf{F F}{ }^{H}\right)=\mathbf{I}$. We assume a zero mean i.i.d. structure for the symbols. The noise power for each stream is $\mathbb{E}\left[\left|\mathbf{n}_{k}\right|^{2}\right]=\sigma^{2}$, so the link SNR is $\rho=P_{t} / \sigma^{2}$, where $P_{t}$ is the total transmit power. The received vector $\mathbf{y}$ is decoded by means of a linear combiner $\boldsymbol{\Omega}$ to produce $\tilde{\mathbf{y}}=\Omega^{H} \mathbf{y}$. We consider three types of receivers, namely SVD receiver, ZF receiver and MMSE receiver. The linear combiner expressions for the SVD, ZF and MMSE receivers in multi-stream MIMO transmission are given by

$$
\begin{gathered}
\boldsymbol{\Omega}_{\mathrm{SVD}}=\mathbf{U} \\
\boldsymbol{\Omega}_{\mathrm{ZF}}=\mathbf{H F}\left(\mathbf{F}^{H} \mathbf{H}^{H} \mathbf{H F}\right)^{-1}
\end{gathered}
$$

and

$$
\boldsymbol{\Omega}_{\mathrm{MMSE}}=\mathbf{H F}\left(\frac{L}{\rho} \mathbf{I}+\mathbf{F}^{H} \mathbf{H}^{H} \mathbf{H F}\right)^{-1},
$$

respectively, where $\mathbf{U}$ represents the left unitary matrix of the channel. Similarly for the single-stream MIMO transmission, the SVD, ZF and MMSE receivers are given by

$$
\begin{gathered}
\mathbf{w}_{\mathrm{SVD}}=\mathbf{u}_{\mathrm{dom}} \\
\mathbf{w}_{\mathrm{ZF}}=\mathbf{H} \mathbf{f}\left(\mathbf{f}^{H} \mathbf{H}^{H} \mathbf{H} \mathbf{f}\right)^{-1}
\end{gathered}
$$

and

$$
\mathbf{w}_{\mathrm{MMSE}}=\mathbf{H f}\left(\frac{1}{\rho} \mathbf{I}+\mathbf{f}^{H} \mathbf{H}^{H} \mathbf{H f}\right)^{-1},
$$

respectively, where $\mathbf{u}_{\text {dom }}$ denotes the left unitary vector corresponding to dominant singular value of $\mathbf{H}$. 


\subsubsection{Capacity Loss in ZF and MMSE Recivers}

We now derive the SINR and capacity loss results as a function of the quantization error (in terms minimum squared chordal distance), $d_{\min _{k}}$, for the ZF and MMSE receivers discussed in the previous section. We will focus on the most common scenarios, namely one and two-stream transmission, referred to as Layer 1 (L1) and Layer 2 (L2). The SINR of the $k^{\text {th }}$ stream in the ZF receiver is given by [10] [89] [68]

$$
\mathrm{SINR}_{k}=\frac{\rho}{L\left[\left(\mathbf{F}^{H} \mathbf{H}^{H} \mathbf{H F}\right)^{-1}\right]_{k, k}},
$$

which can be written as

$$
\mathrm{SINR}_{k}=\frac{\rho}{L\left[\left(\mathbf{F}^{H} \mathbf{V} \boldsymbol{\Lambda} \mathbf{V}^{H} \mathbf{F}\right)^{-1}\right]_{k, k}}
$$

where $\Lambda$ is a diagonal matrix containing the eigenvalues of the matrix $\mathbf{H}^{H} \mathbf{H}$.

\section{Two Stream Transmission (L2)}

In this subsection, we derive the capacity loss expression at high SNR for the ZF and MMSE receivers with L2 transmission. We can write the SINR of the $k^{\text {th }}$ stream for the ZF receiver in (4.15) as

$$
\mathrm{SINR}_{k}=\frac{\rho}{L} \frac{1}{\left(\left[\begin{array}{cc}
\sum_{i=1}^{m} \lambda_{i}\left|\mathbf{v}_{i}^{H} \mathbf{f}_{1}\right|^{2} & \sum_{i=1}^{m} \lambda_{i} \mathbf{v}_{1}^{H} \mathbf{f}_{i} \mathbf{f}_{i}^{H} \mathbf{v}_{2} \\
\sum_{i=1}^{m} \lambda_{i} \mathbf{v}_{2}^{H} \mathbf{f}_{i} \mathbf{f}_{i}^{H} \mathbf{v}_{1} & \sum_{i=1}^{m} \lambda_{i}\left|\mathbf{v}_{i}^{H} \mathbf{f}_{2}\right|^{2}
\end{array}\right]_{k, k}\right.}
$$

where $\lambda_{i}$ denotes the $i^{\text {th }}$ eigenvalue of $\mathbf{H}^{H} \mathbf{H}$. Taking the inverse in (4.16), we can, after some manipulation, express the SINR of the ZF receiver with L2 transmission as

$$
\mathrm{SINR}_{k}=\frac{\rho}{L} \sum_{i=1}^{m} \lambda_{i}\left|\alpha_{k, i}\right|^{2}-\left[\frac{\left|\sum_{i=1}^{m} \lambda_{i} \alpha_{k, i} \alpha_{q, i}^{H}\right|^{2}}{\sum_{i=1}^{m} \lambda_{i}\left|\alpha_{q, i}\right|^{2}}\right]_{\substack{q=1,2 \\ q \neq k}}
$$


where we have defined $\alpha_{k, i}=\mathbf{f}_{k}^{H} \mathbf{v}_{i}$. We can approximate (4.17) by disregarding the second term as it is relatively a smaller value, giving

$$
\mathrm{SINR}_{k} \approx \frac{\rho}{L} \sum_{i=1}^{m} \lambda_{i}\left|\alpha_{k, i}\right|^{2}
$$

In the high SNR regime, the performance of the ZF and MMSE receivers are similar [10], therefore, (4.17) and (4.18) are also valid for the MMSE receiver with L2 transmission. Isolating the $k^{\text {th }}$ stream in the summation in (4.18) and using (4.5) we can express (4.18) in terms of $d_{\min _{k}}$ as

$$
\operatorname{SINR}_{k_{(\mathrm{ZF} / \mathrm{MMSE})}} \approx \frac{\rho}{L}\left[\lambda_{k}\left(1-d_{\min _{k}}\right)+\sum_{i=1, i \neq k}^{m} \lambda_{i}\left|\alpha_{k, i}\right|^{2}\right] .
$$

The capacity for the linear receivers is given in terms of their respective stream SINR values as

$$
C_{\text {MIMO-SM }}=\sum_{k=1}^{L} \log _{2}\left(1+\mathrm{SINR}_{k}\right) .
$$

We define capacity loss (CL) for all receivers as the difference between the capacity with perfect feedback and the capacity with codebook feedback. Hence,

$$
\mathrm{CL}=\sum_{k=1}^{L}\left[\log _{2}\left(1+\rho \lambda_{k} / L\right)-\log _{2}\left(1+\mathrm{SINR}_{k}\right)\right] .
$$

The total capacity loss for the ZF and MMSE receivers at high SNR with L2 transmission can be approximated using (4.17) as

$$
\begin{aligned}
& \mathrm{CL}_{(\mathrm{ZF} / \mathrm{MMSE})} \approx \\
& \sum_{k=1}^{2}-\log _{2}\left[1-d_{\min _{k}}+\frac{1}{\lambda_{k}} \times\left(\sum_{i=1, i \neq k}^{m} \lambda_{i}\left|\alpha_{k, i}\right|^{2}-\frac{\left|\sum_{i=1}^{m} \lambda_{i} \alpha_{k, i} \alpha_{q, i}^{H}\right|^{2}}{\sum_{i=1}^{m} \lambda_{i}\left|\alpha_{q, i}\right|^{2}}\right]\right] \\
& \substack{q=1,2 \\
q \neq k} \\
&
\end{aligned}
$$

A simplified expression can be obtained by substituting (4.19) in (4.21), giving

$$
\mathrm{CL}_{(\mathrm{ZF} / \mathrm{MMSE})} \approx \sum_{k=1}^{2}-\log _{2}\left[1-d_{\min _{k}}+\frac{1}{\lambda_{k}} \sum_{i=1, i \neq k}^{m} \lambda_{i}\left|\alpha_{k, i}\right|^{2}\right] .
$$


The approximate of the capacity loss derived here is plotted against the actual capacity loss in Section 4.2.4.

\section{Single Stream Transmission (L1)}

In the case of L1 transmission, the second term in (4.17) is zero and the SINR of the $1^{\text {st }}$ stream is the same as (4.18)

$$
\mathrm{SINR}_{1} \approx \frac{\rho}{L}\left[\sum_{i=1}^{m} \lambda_{i}\left|\alpha_{1, i}\right|^{2}\right]
$$

which can be expressed in terms of quantization error as

$$
\operatorname{SINR}_{1 \text { (ZF/MMSE) }} \approx \rho\left(1-d_{\text {min }_{1}}\right) \lambda_{1}
$$

where we neglected the second term in (4.19) because it is comparatively a smaller value. The capacity loss for ZF and MMSE receivers with L1 transmission is obtained by substituting (4.25) in (4.21), which, for high SNR, gives

$$
\mathrm{CL}_{(\mathrm{ZF} / \mathrm{MMSE})} \approx-\log _{2}\left(1-d_{\min _{1}}\right) .
$$

\subsubsection{Capacity Loss in SVD Reciever}

The SVD receiver in a MIMO channel is more susceptible to inter stream interference as compared to the ZF and MMSE receivers [68]. Here, we present the capacity loss of MIMO systems with SVD receiver in both multi-stream and single-stream transmissions.

\section{Two Stream Transmission (L2)}

For L2 transmission, we can express a received symbol on the $k^{\text {th }}$ stream in (4.6) as

$$
\tilde{y}_{k}=\sqrt{\rho \lambda_{k}} \mathbf{V}^{H}(k) \mathbf{F s}+\tilde{n}_{k},
$$

where $\mathbf{F}=[\mathbf{F}(1) \mathbf{F}(2)], \mathbf{s}=\left[\begin{array}{ll}s_{1} & s_{2}\end{array}\right]^{T}$ and $\tilde{n}_{k}$ is the $k^{t h}$ element of $\tilde{\mathbf{n}}=\mathbf{U}^{H} \mathbf{n}$. From (4.27), we have that the desired signal and inter stream interference for the $1^{\text {st }}$ stream are $\sqrt{\rho \lambda_{1}} \mathbf{V}^{H}(1) \mathbf{F}(1) s_{1}$ and $\sqrt{\rho \lambda_{1}} \mathbf{V}^{H}(1) \mathbf{F}(2) s_{2}$ respectively, and similarly the SINR of the SVD receiver for the $k^{\text {th }}$ stream is

$$
\operatorname{SINR}_{k_{(\mathrm{SVD})}}=\left[\frac{\rho \lambda_{k} \mathbb{E}\left(\left|s_{k}\right|^{2}\right)\left|\mathbf{V}(k)^{H} \mathbf{F}(k)\right|^{2}}{\rho \lambda_{k} \mathbb{E}\left(\left|s_{q}\right|^{2}\right)\left|\mathbf{F}(q)^{H} \mathbf{V}(k)\right|^{2}+\sigma^{2}}\right]_{\substack{q=1,2 \\ q \neq k}} .
$$


We can further simplify (4.28) by using $\mathbb{E}\left(\left|s_{k}\right|^{2}\right)=\mathbb{E}\left(\left|s_{q}\right|^{2}\right)=1 / L$ and $\sigma^{2}=1$. Now (4.28) can be expressed in terms of quantization errors using (4.5) as

$$
\operatorname{SINR}_{k}(\mathrm{SVD})=\left[\frac{1-d_{\min _{k}}}{\left|\alpha_{q, k}\right|^{2}+L / \rho \lambda_{k}}\right]_{\substack{q=1,2 \\ q \neq k}} .
$$

Note that $\mathrm{SINR}_{k_{(\mathrm{SVD})}} \rightarrow\left(1-d_{\min _{k}}\right) /\left|\alpha_{q, k}\right|^{2}$ as $\rho \rightarrow \infty$, so there is an upper limit on the SINR due to interference. This results in a capacity loss which grows with SNR as seen in (4.30). The total capacity loss approximated at high SNR for an SVD receiver with L2 transmission is obtained by using (4.29) in (4.21) to give

$$
\mathrm{CL}_{(\mathrm{SVD})} \approx \sum_{k=1}^{2} \log _{2}\left[\frac{\rho\left|\alpha_{q, k}\right|^{2} \lambda_{k} / L}{\left|\alpha_{q, k}\right|^{2}+1-d_{\min _{k}}}\right]_{\substack{q=1,2 \\ q \neq k}}
$$

\section{Single Stream Transmission (L1)}

In the case of L1 transmission, there is no inter stream interference and $\left|\alpha_{q, k}\right|^{2}=$ 0 for $q \neq k$ in (4.29), giving

$$
\mathrm{SINR}_{1(\mathrm{SVD})} \approx \rho\left(1-d_{\min _{1}}\right) \lambda_{1}
$$

The capacity loss for the SVD receiver in L1 MIMO transmission mode is approximated as

$$
\mathrm{CL}_{(\mathrm{SVD})} \approx-\log _{2}\left(1-d_{\min _{1}}\right) .
$$

Note that the capacity loss expression for the SVD receiver is identical to that of the ZF and MMSE receivers (4.26) for L1 transmission.

\subsubsection{Numerical Results and Discussion}

In this section, we provide capacity loss simulation results for SVD, ZF and MMSE MIMO receivers in Rayleigh i.i.d., spatially correlated Kronecker and wideband WINNER II channels. The MIMO system simulated has four antennas each at transmitter and receiver, where the transmitter uses either L1 or L2 transmission modes. The Kronecker channel is highly correlated with the exponential correlation coefficient $z_{\mathrm{t}}=z_{\mathrm{r}}=0.9$ at both ends.

The total number of OFDM subcarriers are $N_{\text {sub }}=2048$. For each subcarrier, we select a separate codebook entry from the codebook. The codebooks used in the simulation are 4 bit LTE and Grassmannian codebooks. The feedback link is assumed lossless with zero delay. We calculate the ergodic capacity (EC) of the MIMO system by taking the mean of the capacity in (4.20) for all 
the OFDM tones and then averaging it over all the channel realizations, $C_{r}$, such that

$$
\mathrm{EC}=\frac{1}{N_{\mathrm{sub}} C_{r}} \sum_{i=1}^{C_{r}} \sum_{j=1}^{N_{\text {sub }}} \sum_{k=1}^{L} \log _{2}\left(1+\mathrm{SINR}_{k, j, i}\right),
$$

where $\operatorname{SINR}_{k, j, i}$ denotes the SINR of the $k^{\text {th }}$ stream corresponding to the $j^{\text {th }}$ subcarrier and the $i^{\text {th }}$ channel realization. Similarly, the ergodic capacity loss (ECL) is given by

$$
\mathrm{ECL}=\frac{1}{N_{\mathrm{sub}} C_{r}} \sum_{i=1}^{C_{r}} \sum_{j=1}^{N_{\mathrm{sub}}} \sum_{k=1}^{L}\left[\log _{2}\left(1+\rho \lambda_{k, j, i} / L\right)-\log _{2}\left(1+\mathrm{SINR}_{k, j, i}\right)\right]
$$

where $\lambda_{k, j, i}$ is the $k^{\text {th }}$ eigenvalue of $\mathbf{H}_{j, i}^{H} \mathbf{H}_{j, i}$, where $\mathbf{H}_{j, i}$ is the MIMO channel for the $j^{\text {th }}$ subcarrier and the $i^{\text {th }}$ channel realization. The total power gain satisfies $\mathbb{E}\left[\operatorname{tr}\left(\mathbf{H}_{j, i}^{H} \mathbf{H}_{j, i}\right)\right]=n_{r} n_{t}$.

The parameters for the WINNER II channel model are given in Table 4.2.

In Fig. 4.5, we plot the cumulative distribution function (CDF) of the quantization error for both L1 and L2 transmissions with LTE and Grassmannian codebooks in the WINNER II channel. The quantization error is slightly smaller in L1 transmission with the LTE codebook compared to the Grassmannian codebook. However, for L2 transmission, the Grassmannian codebook yields a much smaller quantization error than the LTE codebook. The average quantization error, $d_{\min _{k}}$, given in (4.5), for L1 and L2 transmission with all three channels and both the codebooks is given in Table 4.3.

In Fig. 4.6, EC results for all three receivers with the LTE codebook are presented for different channels. The correlated channels improves the EC in

Table 4.2: WINNER II Simulation Parameters

\begin{tabular}{|l|c|}
\hline Scenario & UMa \\
\hline Frequency Band & $2.1 \mathrm{GHz}$ \\
\hline Bandwidth & $20 \mathrm{MHz}$ \\
\hline Propagation & NLoS \\
\hline Antenna configuration & $4 \mathrm{x} 4$ slant pol \\
\hline Antenna spacing & $4 \lambda_{0} @ \mathrm{Tx}, 0.5 \lambda_{0} @ \mathrm{Rx}$ \\
\hline Receiver speed & $5 \mathrm{~km} / \mathrm{h}$ \\
\hline Sampling density & $4 \mathrm{samples}$ per $\lambda_{0}$ \\
\hline
\end{tabular}

Table 4.3: Average Quantization Errors

\begin{tabular}{|c|c|c|c|c|c|c|}
\hline & \multicolumn{2}{|c|}{ WINNER II } & \multicolumn{2}{c|}{ i.i.d. } & \multicolumn{2}{c|}{ Kronecker } \\
\cline { 2 - 7 } & LTE & Grass. & LTE & Grass. & LTE & Grass \\
\hline $\mathrm{d}_{\text {min }}$ L1 & 0.28 & 0.30 & 0.34 & 0.30 & 0.18 & 0.30 \\
\hline $\mathrm{d}_{\text {min }}$ L2 & 1.43 & 0.90 & 1.45 & 0.90 & 1.44 & 0.90 \\
\hline
\end{tabular}




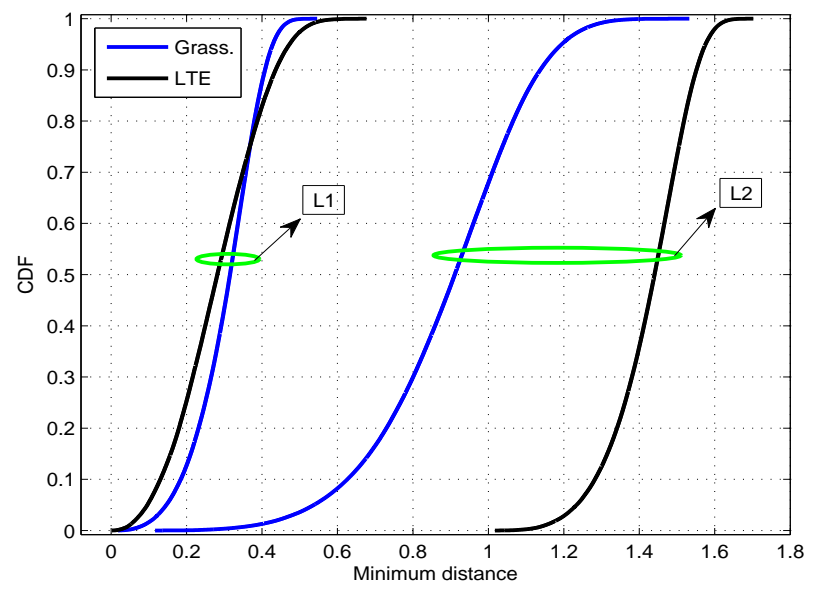

Figure 4.5: Quantization error CDF with LTE and Grassmannian (4 bit) codebooks for L1 and L2 transmission in the WINNER II channel.

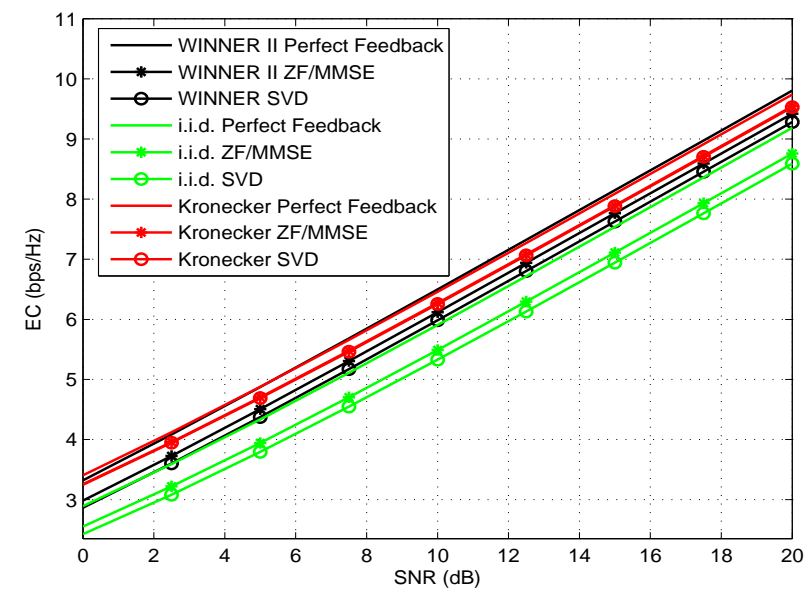

Figure 4.6: Ergodic capacity for L1 transmission with LTE (4 bit) codebook.

the L1 transmission as the dominant eigenmode becomes more dominant in correlated channels. Therefore, the WINNER II and Kronecker channels provide better capacity results than the i.i.d. channel in an L1 transmission.

Figure 4.7 shows the ECL for the L1 transmission with the LTE codebook for WINNER II and Kronecker channels. The approximated capacity loss is calculated using (4.32). It is seen that (4.32) approximates the SVD receiver ECL accurately at high SNR, but a small offset between the actual and approximated ECL for the ZF and MMSE receivers is the result of ignoring the contribution towards signal power from the other unused layers.

In Fig. 4.8, we provide the EC results for all three receivers with an L2 transmission in the WINNER II channel. For moderate SNR, the EC with the Grassmannian codebook is larger than the EC with the LTE codebook for both ZF and MMSE receivers. At high SNR, EC for the SVD receiver is the 


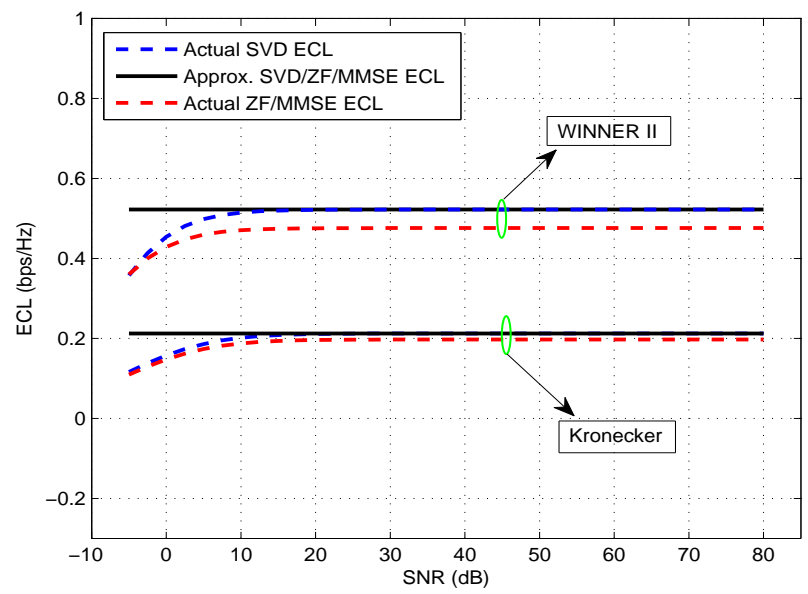

Figure 4.7: Ergodic capacity loss for SVD, ZF and MMSE receivers with L1 transmission and LTE (4 bit) codebook in WINNER II and Kronecker channels.

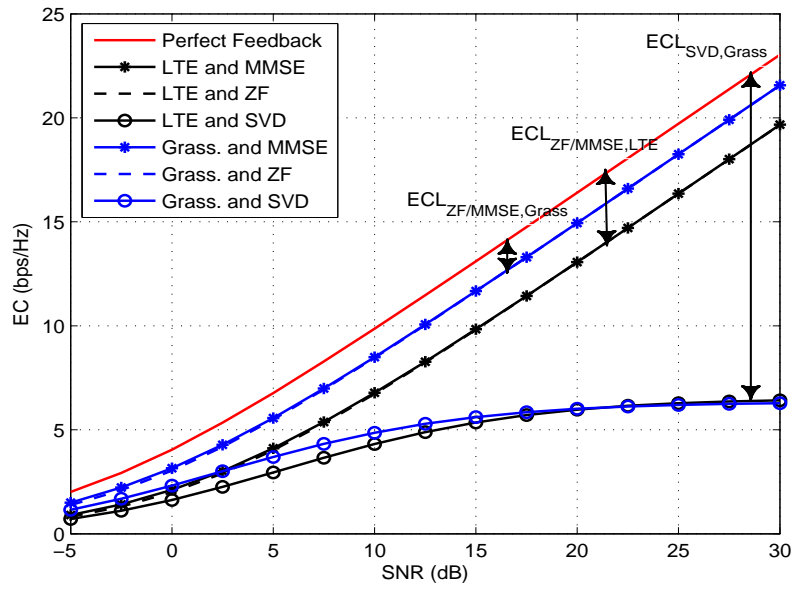

Figure 4.8: Ergodic capacity for L2 transmission with LTE and Grassmannian (4 bit) codebooks in the WINNER II channel.

same for both codebooks. Fig. 4.9 shows the EC results for all three receivers with L2 transmission in the Kronecker channel. The Kronecker channel is highly correlated with $z_{\mathrm{t}}=z_{\mathrm{r}}=0.9$ and it is seen that at low SNR there is a significant difference between the EC of the ZF and MMSE receivers. In the high SNR regime, the $\mathrm{EC}$ of the $\mathrm{ZF}$ and MMSE receivers is equal for both codebooks. The LTE codebook gives better capacity results than the Grassmannian codebook for the SVD receiver at high SNR.

Figure 4.10 shows the ECL for the ZF and MMSE receivers in the case of L2 transmission. As we are not including the effect of interference from other unused layers in (4.23), the approximate ECL values have a $13 \%$ and $28 \%$ error with respect to actual ECL values for LTE and Grassmannian codebooks, respectively. On the other hand, (4.22) approximates the capacity loss perfectly 


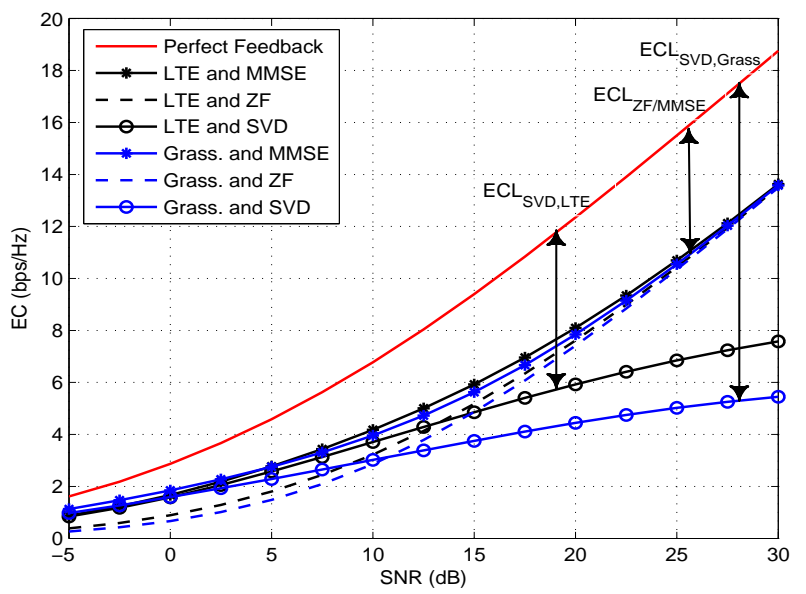

Figure 4.9: Ergodic capacity for L2 transmission with LTE and Grassmannian (4 bit) codebooks in the Kronecker channel $\left(z_{\mathrm{t}}=z_{\mathrm{r}}=0.9\right)$.

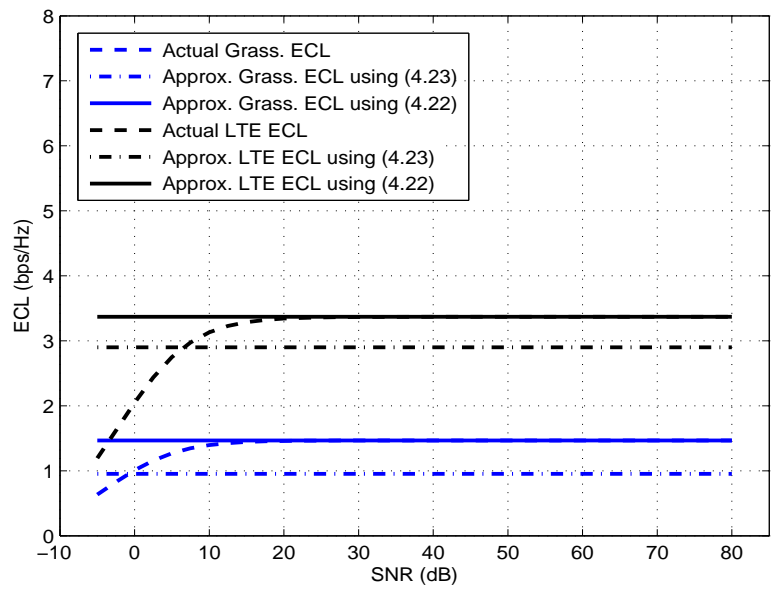

Figure 4.10: Ergodic capacity loss for ZF and MMSE receivers with L2 transmission and LTE and Grassmannian (4 bit) codebooks in the WINNER II channel.

at high SNR for both the codebooks.

In Fig. 4.11, the ECL for the SVD receiver is shown with L2 transmission in the WINNER II channel. The approximated capacity loss is calculated using (4.30). In the case of L2 transmission, the use of (4.30) in the high SNR regime provides the best ECL approximation for the SVD receivers. The ECL for the SVD receiver increases with the SNR in L2 transmission, whereas the ECL for the ZF and MMSE receivers remains constant in the high SNR regime.

\section{Effects of Spatial Correlation on Capacity}

We note that the single-stream MIMO yields better capacity results when correlation is high with Kronecker channels. This is due to the fact that the dominant 


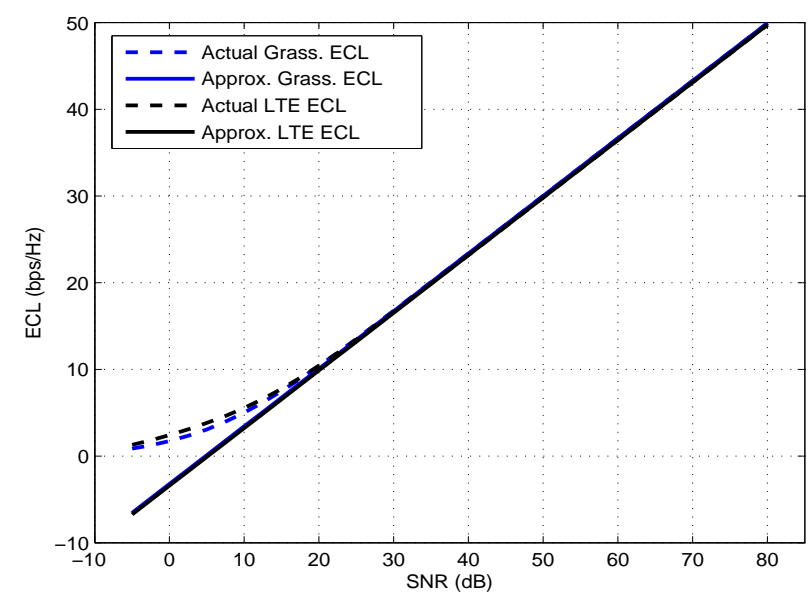

Figure 4.11: Ergodic capacity loss for SVD L2 transmission with LTE and Grassmannian (4 bit) codebooks in the WINNER II channel.

singular value of the channel becomes more dominant, compared to the i.i.d. Rayleigh channel. However, in multi-stream MIMO transmission, as the spatial correlation increases at the transmitter and the receiver, the variance of $\|\mathbf{H}\|_{F}^{2}$ increases, leading to a reduced diversity order [1]. Therefore, with uniform power allocation, the capacity of the MIMO Kronecker channel (2.8) decreases with the increase in the magnitude of the spatial correlation at the transmitter and the receiver [1].

\section{Extension to MU Systems}

The results presented and discussed in this section are for SU MIMO systems. The challenge of extending limited feedback SU system to MU system is that the feedback requirement increases because IUI is not canceled/suppressed properly with quantized channel information at the BS. It is shown in [27] that for MU MISO systems with ZF precoding, the feedback requirement increases as the number of transmit antennas and the SNR increases. The users do not have the information of the other user's channel during quantization, therefore in MU MISO systems the user only quantizes its own channel, like SU systems. However, in MU MISO systems [27], the user quantizes the CDI, whereas in SU MIMO systems (discussed in this chapter) the dominant vectors of the right singular matrix of the channel are quantized. In the following chapters, we consider a MU MISO system where CDI is quantized at the user using codebooks. 


\subsection{Summary}

In this chapter, we have discussed MIMO capacity gains relative to SISO capacity in terms of ratio and difference. We have also shown how the gains are effected at high and low SNR values with different antenna configurations.

We have derived an approximate expression for the capacity loss for a MIMO system that uses multilayer transmission based on codebook beamforming. The capacity loss approximation is expressed in terms of the quantization error between the selected codebook entry and the corresponding channel values and is valid for high SNR. The accuracy of this approximation is compared for single-stream L1 and two-stream L2 transmission modes via simulation of three kinds of channels; a Rayleigh i.i.d. channel, a spatially correlated Kronecker channel and an industry standard WINNER II channel and for three types of receivers (ZF, MMSE and SVD). The approximation is in agreement for both layers for the SVD receiver but slightly under-estimates the loss for the other receiver types, especially for two-stream L2 transmission. In this regard, it could be used as a lower bound. However, in practice, one is likely to only use single-stream L1 transmission for which the loss approximation is good. The approximation therefore provides a useful measure to relate the capacity loss to codebook design in SU MIMO systems. 


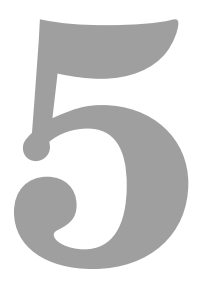

\section{MU MISO Systems with Differential Codebooks}

As discussed in the previous chapter, the quality of the CSI at the transmitter plays an important role in determining the capacity performance for SU systems. This is also the case with MU systems, where multiple users are served simultaneously. The multiple users sharing time and frequency resources interfere with each other, degrading the overall system performance. In MU systems, CSI is critical for transmit precoding and interference mitigation or suppression.

Majority of the codebooks developed for SU MIMO systems with singlestream transmission are also applicable to MU MISO systems, as each user quantizes the channel independently. The quantization of the channel using codebooks can be made effective by taking advantage of the correlation in the channel. Real-world MIMO channels are both spatially and temporally correlated. Depending on the level of spatial and temporal correlation, a codebook can be designed accordingly. Such codebooks have the ability to adjust (at the transmitter and the receiver) according to the changing channel after each feedback interval. These codebooks are referred as differential codebooks in this thesis.

In this chapter, two differential codebook designs are investigated namely: spherical-cap and double-cap based differential codebooks.

\subsection{Motivation and Related Work}

Present day wireless communication systems are not limited to SU scenarios. The majority of systems are designed to support more than one active user. In 
cellular communications, users communicate to the BS on both downlink and uplink. In this thesis, we only investigate the downlink broadcast.

To achieve MIMO gains in a MU setting, multiple users must be served in the same time/frequency resource, as each user has fewer antennas than has at the BS. However, using the same time/frequency resource results in interference at the user, originating from other users signals. Serving multiple users gives rise to MU diversity [23-25] that helps to improve the overall capacity of the system.

In [27], the spectral efficiency loss due to quantized CDI is derived for MU MISO system with the RVQ codebook and ZF precoding. It is mentioned that feedback rate must increase linearly with SNR to achieve full multiplexing gain. In MU MIMO systems, it is shown in [90] that multiple receive antennas provide spectral efficiency improvement by combining received signals. The concept of block diagonalization with limited feedback MU MIMO is given in [91]. The multi-stream MU MIMO broadcast with limited feedback is investigated in [92] with random beamforming.

Most of the work discussed so far considers i.i.d. Rayleigh fading channels. For spatially correlated channels, there are many studies that deal with limited feedback MU multiple antenna systems. In [93], it is mentioned that spatial correlation due to the transmitter is helpful to MU MISO systems, if codebooks are designed accordingly. According to [93], DFT codebooks are useful under spatially correlated channels and outperform Grassmannian codebooks, due to their robust codebook structure. However, DFT codebooks only perform well for correlated ULA scenarios. In [94], the effect of spatial correlation on the performance of RVQ codebooks for MU MISO systems is studied. The codebook structures for spatially correlated MISO channels are also studied in [46,95]. For MU MIMO systems, the codebook designs for spatially correlated channels are investigated in $[96,97]$.

For temporally correlated channels, Grassmannian predictive frameworks for limited feedback MU MISO systems are discussed in [98-101], where the main idea is to exploit the differential geometric structure of the Grassmannian manifold. In [102], a differential feedback scheme is presented that updates the previous CSI at the BS using a spectral efficiency loss metric and a Gaussian quantization error assumption. A polar-cap differential codebook design for a temporally and spatially correlated MISO channel is discussed in [46]. In the polar-cap structure, one codeword is at the center of the cap (also called the basis vector) and all other codewords are on the circumference of the cap (located at the same distance from the basis vector). This codebook is rotated to be centered on the previously selected codeword after each feedback and outperforms the rotation-based codebook [103]. The disadvantage of [46] is that it requires the feedback process to be reset after a few feedback intervals, es- 
pecially when using the adaptive scaling method. The adaptive scaling in [46] continually shrinks the radius of the codebook as the channel evolves, where the radius of the cap is defined as the distance between the basis vector to the furthest codeword in the cap. After a while, the codebook radius becomes too small to successfully track the channel and this issue is handled by resetting the codebook to the base codebook. A differential codebook is developed in [104] for dual-polarized MISO channels that are rotation-based and has shown to achieve better spectral efficiency than 3GPP LTE codebooks even with smaller codebook size. In IEEE $802.16 \mathrm{~m}$ wireless communication standards, two differential codebook based limited feedback schemes are considered that are based on rotation $[105,106]$ and transformation [107, 108]. In [109], an adaptive codebook has been investigated that tracks slowly varying MIMO channels. It also adapts to the varying statistics of the channel. The operations necessary for codebook adaptation such as rotation and scaling are also discussed in [109].

Motivated by [109], we develop two differential codebook designs based on spherical-cap [110,111] and double-cap [112] structures. The structure and working of these differential codebooks are explained in this chapter. Both differential codebook designs require rotation and scaling operations in order to maneuver the codebook entries (codewords). We also introduce an SVD based rotation of the codewords.

Adaptive scaling of the codebooks after every feedback interval is crucial to adjust the differential codebooks cap radii, as it controls the codeword packing density inside the codebook. We discuss two adaptive scaling methods [110,111] for the spherical-cap differential codebook. An adaptive scaling method for the double-cap differential codebook [112] is also investigated that jointly scales the two caps after each feedback interval. We evaluate the performance of these differential codebooks in a MU setting, therefore we rely on ZF and SLNR precoding schemes at the transmitter to mitigate/suppress interference.

Some of the adaptive scaling techniques discussed in this chapter assume that the long-term channel statistics (such as spatial and temporal correlations) are known at the receiver, through an estimation or a negligible signaling overhead.

\subsection{Downlink System Description}

Consider a single cell MU MISO system with a single BS serving $K$ singleantenna users. The number of transmit antennas at the BS is $n_{t}$ and we assume here that $K \leq n_{t}{ }^{1}$. In this chapter, we do not consider path loss and shadowing. On the downlink, the received signal at user $k$ for the transmitted data symbol,

\footnotetext{
${ }^{1} \mathrm{ZF}$ precoding is constrained by the fact that the number of users must be equal to or less than number of transmit antennas for perfect interference nulling. Therefore, here we consider the case where $K \leq n_{t}$. Also, we do not consider scheduling of the users.
} 
$s_{k}$, is given by

$$
y_{k}=\sqrt{\frac{\rho}{K}}\left(\overline{\mathbf{h}}_{k} \mathbf{w}_{k}\right) s_{k}+\sqrt{\frac{\rho}{K}} \sum_{j=1, j \neq k}^{K}\left(\overline{\mathbf{h}}_{k} \mathbf{w}_{j}\right) s_{j}+n_{k},
$$

where $\overline{\mathbf{h}}_{k}$ denotes the $1 \times n_{t}$ correlated channel vector between the BS and the user $k$. The precoding vector of size $n_{t} \times 1$ for the $k^{\text {th }}$ user is denoted by $\mathbf{w}_{k}$ and $n_{k}$ is a noise term at the $k^{\text {th }}$ user, modeled as $n_{k} \sim \mathcal{C N}\left(0, \sigma_{k}^{2}\right)$, where, $\sigma_{k}^{2}$ is the noise variance at the $k^{\text {th }}$ user. The SNR is given by $\rho=P_{t} / \sigma_{k}^{2}$, where $P_{t}$ is the total transmit power. We assume equal noise variance and average power for all users. The data symbols and precoding vector are normalized such that $\mathbb{E}\left[\left|s_{k}\right|^{2}\right]=\left\|\mathbf{w}_{k}\right\|^{2}=1, \forall k$. The spectral efficiency of the MU MISO system is given by (3.6).

\subsection{Spherical-Cap Differential Codebook}

In this section, we discuss the spherical-cap design for the differential codebook. The spherical-cap design was originally proposed for spatially correlated MIMO channels in [45].

In order to explain the spherical-cap structure, we begin by discussing the concepts of the Grassmannian manifold and the Grassmannian line packing (GLP) problem. Let $\mathbf{x}$ denote the unit vector in $\mathbb{C}^{n_{t}}$. The set of unit norm vectors in $\mathbb{C}^{n_{t}}$ lie in a unit sphere space also called the one-dimensional complex Stiefel manifold, $\Omega_{n_{t}}$, given by [45]

$$
\Omega_{n_{t}}=\left\{\mathbf{x} \in \mathbb{C}^{n_{t}}:\|\mathbf{x}\|=1\right\}
$$

Let $\mathbf{x}_{1}$ and $\mathbf{x}_{2}$ be the two unit norm vectors in $\Omega_{n_{t}}$ for which an equivalence relation between these two unit vectors is given by $\mathbf{x}_{1}=\mathrm{e}^{j \phi} \mathbf{x}_{2}$ for some $\phi \in$ $[0,2 \pi)[42]$. This means that the two vectors are equivalent if they are on the same plane in $\mathbb{C}^{n_{t}}[42,113]$. The Grassmannian manifold is the set of all onedimensional subspaces in $\mathbb{C}^{n_{t}}$.

According to [45], the phase invariance of vector $\mathbf{x}$ (the performance of the beamforming is independent of the phase of the beamforming vector) can be incorporated by considering vectors as points on the Grassmannian manifold $G\left(n_{t}, 1\right)$. Therefore, the Grassmannian line packing (GLP) problem of finding the set of $N_{c}=2^{B}$ lines in $\mathbb{C}^{n_{t}}$ having maximum distance between any pair of lines [42] can be used to design the codebook for i.i.d. Rayleigh channels. Using chordal distance metric on $G\left(n_{t}, 1\right)$, a spherical-cap on $G\left(n_{t}, 1\right)$ with center, $\mathbf{c}$, 
Grassmannian codebook

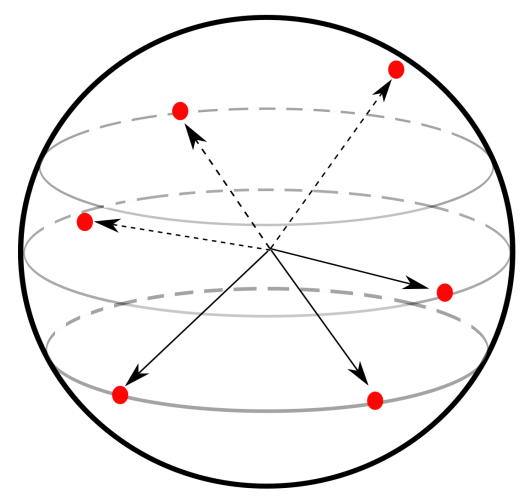

Spherical-cap differential codebook

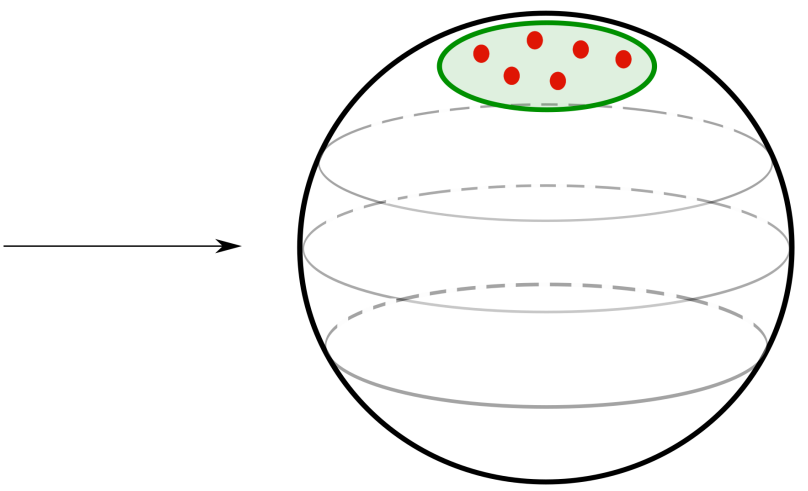

Figure 5.1: Transformation of Grassmannian codebook to spherical-cap differential codebook.

and radius, $\varsigma$, can be defined as [45]

$$
\mathbb{O}(\mathbf{c}, \varsigma)=\left\{\mathbf{x} \in G\left(n_{t}, 1\right): \sqrt{1-\left|\mathbf{c}^{H} \mathbf{x}\right|^{2}}<\varsigma\right\}
$$

The transformation of the Grassmannian codebook to the spherical-cap differential codebook is illustrated in Fig. 5.1.

For the MU MISO system considered in this chapter, each user generates its spherical-cap differential codebook according to the method in (5.3). The spherical cap is centered on $\mathbf{c}=[1,0, \cdots, 0]^{T}$. A spherical cap of size $2^{B}$ on $G\left(n_{t}, 1\right)$ with center $\mathbf{c}$ and radius, $\varsigma$, is denoted by $\mathbb{O}_{k}(\mathbf{c}, \varsigma)$, such that $\mathbb{O}_{k}(\mathbf{c}, \varsigma)=$ $\left\{\mathbf{f}_{k}^{(i)} \in G\left(n_{t}, 1\right): \sqrt{1-\left|\mathbf{c}^{H} \mathbf{f}_{k}^{(i)}\right|^{2}}<\varsigma\right\}, \forall i$. Each codeword is scaled individually [45] to give the spherical cap differential codebook, given by

$$
\mathbf{F}_{\text {spherical }}=\left\{\left[\begin{array}{c}
\sqrt{1-\alpha^{2}\left(1-r_{1,1}^{2}\right)} e^{j \theta_{1,1}} \\
\vdots \\
\alpha r_{n_{t}, 1} e^{j \theta_{n_{t}, 1}}
\end{array}\right], \cdots,\left[\begin{array}{c}
\sqrt{1-\alpha^{2}\left(1-r_{1,2^{B}}^{2}\right)} e^{j \theta_{1,2} B} \\
\vdots \\
\alpha r_{n_{t}, 2^{B}} e^{j \theta_{n_{t}, 2^{B}}}
\end{array}\right]\right\},
$$

where $r_{m, n} e^{j \theta_{m, n}}$ is the polar form of the $m^{\text {th }}$ entry of the $n^{\text {th }}$ GLP codeword and $\alpha$ is a scaling parameter satisfying $0<\alpha<1$. After scaling operation, all the codewords in the spherical cap lie inside the radius, $\alpha$.

\subsection{Feedback Procedure}

The feedback steps are as follows:

- For the first feedback at $t=0$, the user $k$ selects an appropriate codeword $\overline{\mathbf{q}}_{k}$ (discussed in Chapter 3, Section 3.4) from a base codebook (e.g. RVQ, 
Grassmannian or LTE) and feeds back the index of this selected codeword to the BS.

- For the second feedback period denoted by $t=1$, both BS and user $k$ use spherical-cap differential codebook centered on the previous selected codeword (from the base codebook).

- For the rest of the feedback periods, the process continues with the differential codebook with fixed or adaptive scaling. The adaptive scaling methods are discussed later, where the main idea is to vary the value of the scaling parameter, $\alpha$ in (5.4), after each feedback interval.

\subsection{Tracking Channel with Spherical-Cap}

In this section, we discuss the tracking of the correlated channel with the spherical-cap differential codebook over time. The spherical-cap differential codebook feedback approach for time $t=1$ (first feedback) and $t=2$ (second feedback) is depicted in Fig. 5.2. It is shown that the selected codeword at time $t=0$ (from the base codebook) is the center of the spherical-cap differential codebook at time $t=1$. The user selects the codeword (represented by the red dot) that is closest to the actual channel (represented by orange color). For the next feedback time $t=2$, the selected codeword at $t=1$ becomes a new center of the spherical cap differential codebook using the rotation technique discussed in Section 5.6. Therefore, once the codebook starts following the channel, the quantization errors also reduce. The reason is that the spherical-cap takes advantage of the adaptive scaling techniques (discussed later) to adjust the radius of the cap over time, for tracking the channel.
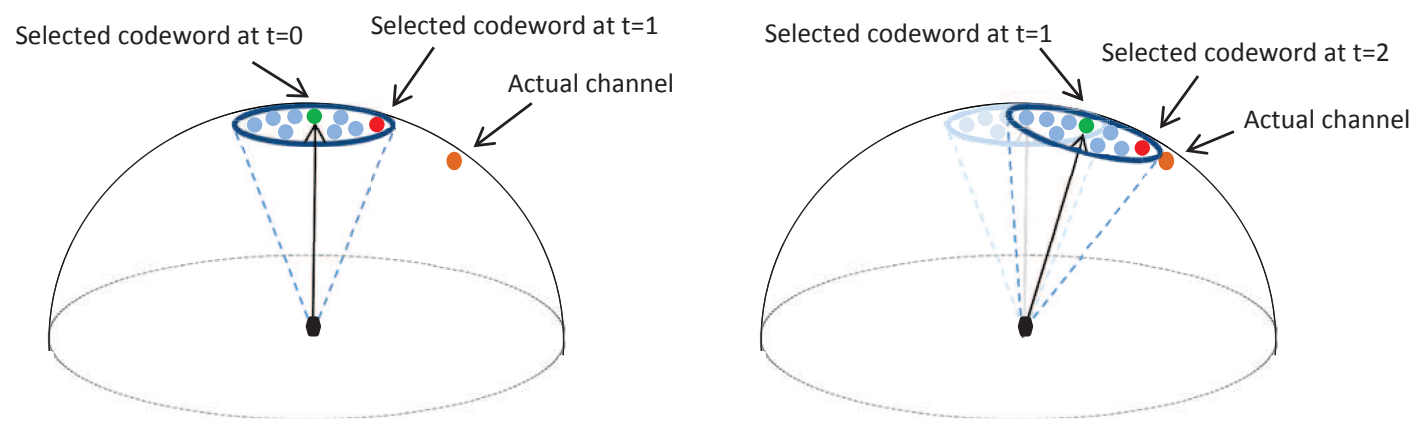

Figure 5.2: Structure of the spherical-cap differential codebook at time, $t=1$ and $t=2$. 


\subsection{Rotation Operation on Codebooks}

In this section, we introduce an SVD-based rotation operation that can effectively rotate the codewords in the codebook to the desired location. For clarity, we drop the user index $k$ and time index $t$. To rotate a codebook, we first perform SVD operation on the codewords, given by

$$
\operatorname{SVD}\left(\overline{\mathbf{q}} \mathbf{c}^{H}\right)=\mathbf{U D V}^{H}
$$

where, $\mathbf{c}$ is the center of the cap and $\overline{\mathbf{q}}$ is the current selected codeword given by (3.14). $\mathbf{D}$ is a diagonal matrix with zero non-diagonal entries containing only the single singular value which is equal to one. $\mathbf{U}$ and $\mathbf{V}$ are the left and right unitary matrices, respectively. Now, we can define the rotation matrix as $\boldsymbol{\Theta}=\mathbf{U V}^{H}$. Using $\boldsymbol{\Theta}$, the cap center $\mathbf{c}$ can be rotated to $\overline{\mathbf{q}}$, such that $\overline{\mathbf{q}}=\mathbf{\Theta} \mathbf{c}$. As $\overline{\mathbf{q}}$ and $\mathbf{c}$ are orthogonal vectors, therefore $\boldsymbol{\Theta}$ can be expressed as the form given in [45], such that

$$
\boldsymbol{\Theta}=[\overline{\mathbf{q}} \underbrace{\overline{\mathbf{q}}_{1}^{\perp} \ldots \overline{\mathbf{q}}_{n_{t}-1}^{\perp}}_{n_{t}-1 \text { null vectors }}]\left[\begin{array}{ll}
\mathbf{c} & \underbrace{\mathbf{c}_{1}^{\perp} \ldots \mathbf{c}_{n_{t}-1}^{\perp}}_{n_{t}-1 \text { null vectors }}
\end{array}\right]^{H}
$$

The rotation matrix, $\Theta$, is a unitary matrix with determinant equal to one. Once the rotation matrix $\Theta$ is calculated, all the codewords in the codebook are rotated using this rotation matrix that results in a new codebook structure with the center $\overline{\mathbf{q}}$.

\section{7 $\quad$ Scaling Operation on Codebooks}

Differential codebooks can have a fixed or adaptive scaling (corresponding to $\alpha$ in (5.4)). Fixed scaling means that the differential codebook keeps a constant radius of the codebook cap and it does not change throughout the transmission. On the other hand, in adaptive scaling the radius of the codebook varies after each feedback interval depending on factors such as spatial/temporal correlation and quantization errors. For both fixed or adaptive scaling techniques, the scaling to alter the radius of the codebook is performed using (5.4).

\subsubsection{Adaptive Scaling Technique I}

An adaptive scaling technique for the differential codebook that does not require the knowledge of the spatial/temporal correlation statistics at the BS is discussed in this section. We denote the adaptive scaling parameter by $\tilde{\alpha}$. The idea is that after each feedback the codebook is re-scaled using (5.4), however initially for the second feedback (at time $t=1$ ), the cap radius is set to a value 
$\tilde{\alpha}[1]$ [46], known at both transmitter and receiver. The scaling parameter for the successive feedback transmissions, $t>1$, is defined as the chordal distance between the current selected codeword and the previously selected codeword, such that

$\tilde{\alpha}[t+1]= \begin{cases}\sqrt{1-\left|\overline{\mathbf{q}}^{H}[t-1] \overline{\mathbf{q}}[t]\right|^{2}}, & \text { if codeword at the center is not selected } \\ \tilde{\alpha}[t] & \text { if codeword at the center is selected. }\end{cases}$

where $\tilde{\alpha}[t+1]$ is the scaling parameter for the next feedback interval. For clarity, we drop the user index $k$. This method is effective when the differential codebook reset time is small. The disadvantage of this adaptive scaling technique is that the radius of the spherical-cap keeps shrinking after every feedback time as the distance between codewords decreases over time. Therefore, the reset is required where the process begins again with the base codebook.

\subsubsection{Adaptive Scaling Technique II}

Adaptive Scaling Technique I requires resetting of the differential codebook after a few feedback intervals as the spherical-cap radius shrinks to zero over time. This results in large quantization errors if the feedback process is not reset to the base codebook. To address this issue, if long term channel correlation (spatial and temporal) statistics are shared with the BS [103], then adaptive scaling can be used for longer transmission periods without a reset. The temporal and spatial correlation coefficients (i.e., $\epsilon$ and $z_{\mathrm{t}}$ ) are estimated at the user and fed back to the BS. However, in this study, like [46,114], we assume that perfect knowledge of both spatial and temporal channel statistics are available at the BS.

In this section, we explain the Adaptive Scaling Technique II for spatially and temporally correlated channel, $\overline{\mathbf{h}}$, given by (2.14). The Adaptive Scaling Technique II depends on mean quantization error and mean channel variation (i.e., the amount of spatial/temporal correlation in the channel). The mean channel variation is defined as the average chordal distance between the previous and current channel directions, given by (for clarity, we drop the user index $k$ )

$$
\mathrm{d}_{\text {mean }}=\mathbb{E}\left[\sqrt{1-\left|\overline{\mathbf{u}}^{H}[t-1] \overline{\mathbf{u}}[t]\right|^{2}}\right]
$$

Using Jensen's inequality in (5.8) we have

$$
\mathrm{d}_{\text {mean }} \leq \sqrt{1-\mathbb{E}\left[\left|\overline{\mathbf{u}}^{H}[t-1] \overline{\mathbf{u}}[t]\right|^{2}\right]} .
$$

We find the expected value in (5.9) for the channel modeled by the FOGM 
process with both spatial and temporal correlation (2.14) [46]. We can write (5.9) as

$$
\mathbb{E}\left[\left|\overline{\mathbf{h}}^{H}[t-1] \overline{\mathbf{h}}[t]\right|^{2}\right]=\mathbb{E}\left[\left|\overline{\mathbf{h}}^{H}[t-1]\left(\epsilon \overline{\mathbf{h}}[t-1]+\sqrt{1-\epsilon^{2}} \mathbf{y}[t] \mathbf{R}_{\mathrm{t}}^{1 / 2}\right)\right|^{2}\right]
$$

where $\mathbf{y}[t]$ is a $\mathbb{C}^{1 \times n_{t}}$ vector having i.i.d. $\mathcal{C N}(0,1)$ entries and $\mathbf{R}_{\mathrm{t}}$ is a spatial correlation matrix (assumed to follow an exponential model [46]) with spatial correlation coefficient $z_{\mathrm{t}}$. We later show that the $z_{\mathrm{t}}$ value has a minimal effect on the adaptation process. We assume the channel is temporally correlated with a correlation coefficient $\epsilon$, following the Jakes' model $\epsilon=J_{0}\left(2 \pi f_{D} \tau\right)$, where $f_{D}$ is the Doppler frequency. Simplifying (5.10) we note that the expectation of cross terms is zero, therefore we get

$$
\mathbb{E}\left[\left|\overline{\mathbf{h}}^{H}[t-1] \overline{\mathbf{h}}[t]\right|^{2}\right]=\epsilon^{2} \mathbb{E}\left[\|\overline{\mathbf{h}}[t-1]\|^{4}\right]+\left(1-\epsilon^{2}\right) \mathbb{E}\left[\left\|\overline{\mathbf{h}}^{H}[t-1] \mathbf{y}[t] \mathbf{R}_{\mathrm{t}}^{1 / 2}\right\|_{F}^{2}\right] .
$$

Using the independence between amplitude and direction of $\overline{\mathbf{h}}[t]$ and $\overline{\mathbf{h}}[t-1]$ [82], we can approximate $\mathbb{E}\left[\left|\overline{\mathbf{h}}^{H}[t-1] \overline{\mathbf{h}}[t]\right|^{2}\right]$ for spatially and temporally correlated channels with $\epsilon \approx 1$ by

$$
\mathbb{E}\left[\left|\overline{\mathbf{h}}^{H}[t-1] \overline{\mathbf{h}}[t]\right|^{2}\right] \approx \mathbb{E}\left[\left\|\overline{\mathbf{h}}^{H}[t-1]\right\|^{4}\right] \mathbb{E}\left[\left|\overline{\mathbf{u}}^{H}[t-1] \overline{\mathbf{u}}[t]\right|^{2}\right]
$$

Substituting (5.12) in (5.11) and simplifying gives

$$
\mathbb{E}\left[\left|\overline{\mathbf{u}}^{H}[t-1] \overline{\mathbf{u}}[t]\right|^{2}\right] \approx \epsilon^{2}+\left(1-\epsilon^{2}\right) \Psi
$$

where

$$
\Psi \approx \frac{\mathbb{E}\left[\left\|\overline{\mathbf{h}}^{H}[t-1] \mathbf{y}[t] \mathbf{R}_{\mathrm{t}}^{1 / 2}\right\|_{F}^{2}\right]}{\mathbb{E}\left[\left\|\overline{\mathbf{h}}^{H}[t-1]\right\|^{4}\right]}
$$

Noting the fact that $\overline{\mathbf{h}}^{H}[t-1]=\left(\mathbf{y}[t-1] \mathbf{R}_{\mathrm{t}}^{1 / 2}\right)^{H}$, where $\mathbf{y}[t-1]$ is distributed according to $\mathcal{C N}(0,1)$ and substituting in $(5.14)$, we get

$$
\Psi \approx \frac{\mathbb{E}\left[\left\|\mathbf{R}_{\mathrm{t}}^{1 / 2} \mathbf{y}^{H}[t-1] \mathbf{y}[t] \mathbf{R}_{\mathrm{t}}^{1 / 2}\right\|_{F}^{2}\right]}{\mathbb{E}\left[\left(\left\|\mathbf{R}_{\mathrm{t}}^{1 / 2} \mathbf{y}^{H}[t-1]\right\|^{2}\right)^{2}\right]} .
$$

Using the eigenvalue decomposition $\mathbf{R}_{\mathrm{t}}=\boldsymbol{\Phi} \mathbf{O} \boldsymbol{\Phi}^{H}$, where $\boldsymbol{\Phi}$ is an $n_{t} \times n_{t}$ matrix of eigenvectors of $\mathbf{R}_{\mathrm{t}}$ and $\mathbf{O}$ is a diagonal matrix containing the eigenvalues 
$\left(\mathrm{o}_{1}, \mathrm{o}_{2}, \ldots, \mathrm{o}_{n_{t}}\right)$ of $\mathbf{R}_{\mathrm{t}},(5.15)$ can be written as

$$
\Psi \approx \frac{\operatorname{tr}\left(\mathbb{E}\left[\mathbf{O} \tilde{\mathbf{y}}^{H}[t-1] \tilde{\mathbf{y}}[t-1]\right] \mathbb{E}\left[\mathbf{O} \tilde{\mathbf{y}}^{H}[t] \tilde{\mathbf{y}}[t]\right]\right)}{\mathbb{E}\left[\left(\tilde{\mathbf{y}}[t-1] \mathbf{O} \tilde{\mathbf{y}}^{H}[t-1]\right)^{2}\right]} .
$$

where $\tilde{\mathbf{y}}^{H}[t-1]=\boldsymbol{\Phi}^{H} \mathbf{y}^{H}[t-1]$ and $\tilde{\mathbf{y}}[t-1]=\mathbf{y}[t-1] \boldsymbol{\Phi}$ are also distributed according to $\mathcal{C N}(0,1)$. The numerator of (5.16) can be simplified using the fact that off diagonal entries of $\left(\mathbf{O} \tilde{\mathbf{y}}^{H}[t-1] \tilde{\mathbf{y}}[t-1]\right)$ and $\left(\mathbf{O} \tilde{\mathbf{y}}^{H}[t] \tilde{\mathbf{y}}[t]\right)$ are zero mean and entries of the vectors $|\tilde{\mathbf{y}}[t-1]|^{2}$ and $|\tilde{\mathbf{y}}[t]|^{2}$ are exponentially distributed with $\mathbb{E}\left[|\tilde{\mathbf{y}}[t-1]|^{2}\right]=\mathbb{E}\left[|\tilde{\mathbf{y}}[t]|^{2}\right]=1$. This gives

$$
\operatorname{tr}\left(\mathbb{E}\left[\mathbf{O} \tilde{\mathbf{y}}^{H}[t-1] \tilde{\mathbf{y}}[t-1]\right] \mathbb{E}\left[\mathbf{O} \tilde{\mathbf{y}}^{H}[t] \tilde{\mathbf{y}}[t]\right]\right)=\sum_{i=1}^{n_{t}} \mathrm{o}_{i}^{2} .
$$

Similarly, the denominator in (5.16) can be expressed as

$$
\mathbb{E}\left[\left(\tilde{\mathbf{y}}[t-1] \mathbf{O} \tilde{\mathbf{y}}^{H}[t-1]\right)^{2}\right]=\mathbb{E}\left[\left(\sum_{i=1}^{n_{t}} \mathrm{o}_{i}^{2}\left|\tilde{y}^{(i)}[t-1]\right|^{2}\right)^{2}\right],
$$

where $\tilde{y}^{(i)}[t-1]$ denotes the $i^{\text {th }}$ entry of the vector $\tilde{\mathbf{y}}[t-1]$. Expanding the term $\left(\sum_{i=1}^{n_{t}} \mathrm{o}_{i}^{2}\left|\tilde{y}^{(i)}[t-1]\right|^{2}\right)^{2}$ and using the fact that $\mathbb{E}\left[\left|\tilde{y}^{(i)}[t-1]\right|^{4}\right]=2$, we can write (5.16) as

$$
\Psi \approx \frac{\sum_{i=1}^{n_{t}} \mathrm{o}_{i}^{2}}{2 \sum_{i=1}^{n_{t}} \mathrm{o}_{i}^{2}+2 \sum_{\substack{i=1 \\ i<j}}^{n_{t}} \mathrm{o}_{i} \mathrm{O}_{j}}, \quad j=1, \ldots, n_{t} .
$$

Finally, this leads to the approximation

$$
\mathrm{d}_{\text {mean }} \approx \sqrt{1-\epsilon^{2}-\left(1-\epsilon^{2}\right) \Psi} .
$$

For the range of values of $z_{\mathrm{t}}\left(0 \leq z_{\mathrm{t}} \leq 1\right)$, the corresponding values of $\Psi$ have little impact on $\mathrm{d}_{\text {mean }}$ in $(5.20)$, for pedestrian velocities.

Another factor that must also be taken into account before selecting the scaling parameter is the mean quantization error associated with the previous feedback with scaling parameter $\tilde{\alpha}^{*}[t-1]$. Denoting by $\tilde{\alpha}^{*}[t]$ the scaling parameter at time $t$, the upper bound on the mean quantization error at time $t$ is given by [46]

$$
\mathbb{E}\left[1-\left|\overline{\mathbf{q}}^{H}[t] \overline{\mathbf{u}}[t]\right|^{2}\right] \leq\left[\tilde{\alpha}^{*}[t] 2^{\frac{-B}{2\left(n_{t}-1\right)}}\right]^{2} .
$$

The mean quantization error in (5.21) is given in terms of the squared chordal distance. We can have a loose upper bound on the quantization error in terms of the chordal distance by taking the square root of (5.21) and denoting it by 


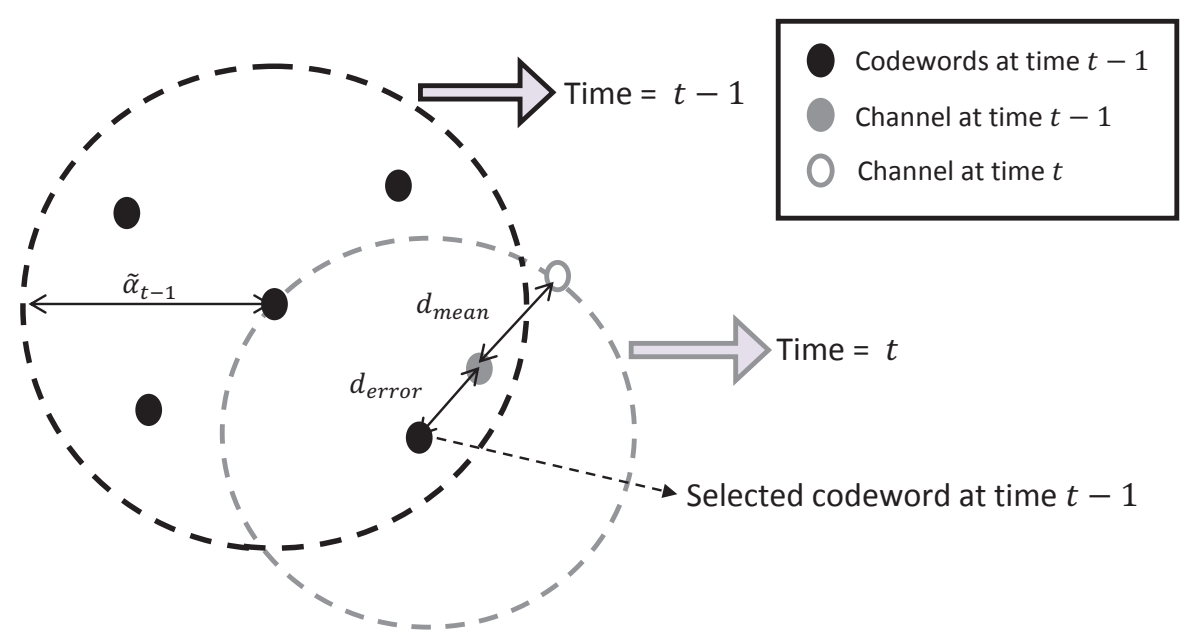

Figure 5.3: The adaptive scaling technique II. Note that $\mathrm{d}_{\text {mean }}$ and $\mathrm{d}_{\text {error }}$ are mean distances and are shown here for illustrative purposes only.

$\mathrm{d}_{\text {error }}$,

$$
\mathrm{d}_{\mathrm{error}} \leq \tilde{\alpha}^{*}[t] 2^{\frac{-B}{2\left(n_{t}-1\right)}}
$$

In order to track the varying channel, we propose the scaling parameter for time $t+1$ to be the sum of (5.20) and (5.22),

$$
\tilde{\alpha}^{*}[t+1] \triangleq \mathrm{d}_{\text {mean }}+\mathrm{d}_{\text {error }}, \quad \text { for } \quad t>1
$$

Starting the process with the base codebook, we initially set the scaling parameter $\tilde{\alpha}^{*}[0]=1$. For the second feedback, the scaling parameter is determined using (5.22) such that $\tilde{\alpha}^{*}[1]=2^{\frac{-B}{2\left(n_{t}-1\right)}}$. The scaling parameter for the successive feedback intervals is calculated using (5.23). This adaptive scaling parameter can be shown to reach an asymptotic value

$$
\tilde{\alpha}_{\infty}^{*}=\mathrm{d}_{\text {mean }}\left[1-2^{\frac{-B}{2\left(n_{t}-1\right)}}\right]^{-1} .
$$

The adaptive scaling technique for time $t-1$ and $t$ is depicted in Fig. 5.3. In the Adaptive Scaling Technique II, the periodic reset of the differential codebook to the base codebook is not required as the radius of the spherical-cap does not shrink to zero with time.

\subsection{Double-Cap Differential Codebook}

The main motivation for designing a double-cap differential codebook is to have a codebook structure for correlated MISO channels that efficiently quantizes the CDI and performs adaptive scaling to keep track of the channel without requiring an additional feedback or a periodic reset. The polar-cap design in [46] 
is not able to track the channel for longer transmission periods as quantization errors becomes large due to shrinkage of the cap radius with time. Therefore, the polar-cap needs to be reset after a few feedback intervals. Also, the adaptive scaling technique presented in [46] requires correlation information at the transmitter to adapt the cap radius over time. In this section, we discuss the double-cap differential codebook [112] capable of running for longer transmission periods without requiring correlation information at the transmitter.

A double-cap differential codebook comprises of two different cap structures with different radii on the Grassmannian manifold, centered around the same location. The double-cap differential codebook consists of an inner spherical cap (discussed in section 5.3) and an outer polar cap [46] structures. The codewords on the circumference of the outer cap are not only used to quantize the channel vector, but also enable rapid tracking of the channel. On the other hand, the inner spherical cap codewords are distributed inside the cap, hence reducing quantization errors once the differential codebook closely follows the channel. As will be shown, splitting the codewords into two distinct subsets allows us to signal the need for the radius adaptation without the additional feedback overhead.

\subsubsection{Double-Cap Design}

The spherical-cap design has already been discussed in Section 5.3. Here, we first look at the polar cap design and then discuss the scaling parameters $\alpha_{p}$ and $\alpha_{s}$ for polar and spherical caps, respectively.

The polar cap differential codebook consists of a basis vector of size $n_{t} \times 1$ given by $\mathbf{c}=[1,0, \cdots, 0]^{T}$ that is also the center of the polar-cap. However, in the double-cap structure, the polar-cap does not include the basis vector as a codeword and all $2^{B}$ codewords are located on the circumference of the polarcap. In order to generate these $2^{B}$ codewords, a Grassmannian codebook [42] on $G\left(n_{t}, 1\right)$, is generated where each codeword is of size $\left(n_{t}-1\right) \times 1$, given by $\mathbf{F}_{k}=\left[\mathbf{f}_{k}^{(1)}, \cdots, \mathbf{f}_{k}^{\left(2^{B}\right)}\right]$, at the $k^{\text {th }}$ user. The next step is to set an appropriate scaling parameter, $\alpha_{p}$, that defines the distance between the basis vector $\mathbf{c}$ and the $2^{B}$ codewords. Once $\alpha_{p}$ is set, then the polar cap differential codebook, $\mathbf{F}_{\text {polar }}$, of size $2^{B}$ is generated by [46]

$$
\mathbf{F}_{\text {polar }}=\left\{\left[\begin{array}{c}
\sqrt{1-\alpha_{p}^{2}} \\
\alpha_{p} \mathbf{f}_{k}^{(1)}
\end{array}\right], \cdots,\left[\begin{array}{c}
\sqrt{1-\alpha_{p}^{2}} \\
\alpha_{p} \mathbf{f}_{k}^{\left(2^{B}\right)}
\end{array}\right]\right\} .
$$

Following the first feedback interval, where a base codebook (e.g. RVQ, Grassmannian or LTE) is used, and the double-cap differential codebook is centered around the selected base codebook codeword, $\overline{\mathbf{q}}_{k}[0]$. Intuitively, it would be appropriate to scale the codebook using knowledge of the quantization error due 
to the base codebook. For this purpose, we assume that the base codebook is the RVQ codebook and use the mean quantization errors result derived in [22]. The initial radius, $\alpha_{s}$, for the inner cap (spherical cap) is set to the average chordal distance between the selected RVQ codeword, $\overline{\mathbf{q}}_{k}$, and the perfect channel direction, $\overline{\mathbf{u}}_{k}$. The outer polar cap radius is initially set to the maximum quantization error limit, $\alpha_{p}=1$. Hence, for smaller errors than the mean quantization error, we use the inner spherical cap for fine tracking of the channel. When the quantization error is larger than the mean quantization error, the outer polar cap codewords are used for quantizing and tracking of the channel. We can write the distance, $\alpha_{s}$, as

$$
\alpha_{s}=\mathbb{E}\left[\sqrt{1-\left|\overline{\mathbf{q}}_{k} \overline{\mathbf{u}}_{k}\right|^{2}}\right] .
$$

We solve the expectation in (5.26) to compute the value of $\alpha_{s}$. As shown in [22], the random variable $X=\sqrt{1-\left|\mathbf{f}_{k}^{(i)} \overline{\mathbf{u}}_{k}\right|^{2}}$ is beta distributed with parameters $2\left(n_{t}-1\right)$ and 1 . If we let $y$ be the minimum of the $2^{B}$ random variables $X$, then the probability density function (PDF) of $y$ can be written as

$$
f(y)=2^{B} f(x)(1-F(x))^{2^{B}-1}, \quad 0<y<1,
$$

where $f(x)$ and $F(x)$ are the PDF and CDF of the independent $X$ variables, given by

$$
f(x)=2\left(n_{t}-1\right) x^{\left(2 n_{t}-3\right)}
$$

and

$$
F(x)=x^{2\left(n_{t}-1\right)} .
$$

Substituting (5.28) and (5.29) in (5.27), we have

$$
f(y)=2^{B+1}\left(n_{t}-1\right) y^{\left(2 n_{t}-3\right)}\left(1-y^{2\left(n_{t}-1\right)}\right)^{2^{B}-1} .
$$

Using $f(y)$, we can calculate the value of $\alpha_{s}$ in (5.26) as

$$
\begin{aligned}
\alpha_{s} & =\int_{0}^{1} y f(y) d y \\
& =2^{B+1}\left(n_{t}-1\right) \int_{0}^{1} y^{2\left(n_{t}-1\right)}\left(1-y^{2\left(n_{t}-1\right)}\right)^{2^{B}-1} d y \\
& =2^{B} \beta\left(\frac{n_{t}-\frac{1}{2}}{n_{t}-1}, 2^{B}\right) .
\end{aligned}
$$

where $\beta(\cdot, \cdot)$ denotes a Beta function. A bound for the squared chordal distance is derived in [27].

We set the radius of the inner cap (spherical cap) to $\alpha_{s}$ initially, given in 
(5.33). The double-cap differential codebook structure is obtained by generating a spherical cap with radius $\alpha_{s}$ and a polar cap with radius $\alpha_{p}$. This double-cap differential codebook is centered around the basis vector, c (not a codeword), and it is used for the second feedback interval with $\alpha_{p}$ and $\alpha_{s}$. The double-cap differential codebook design is shown in Fig. 5.4. The number of codewords is

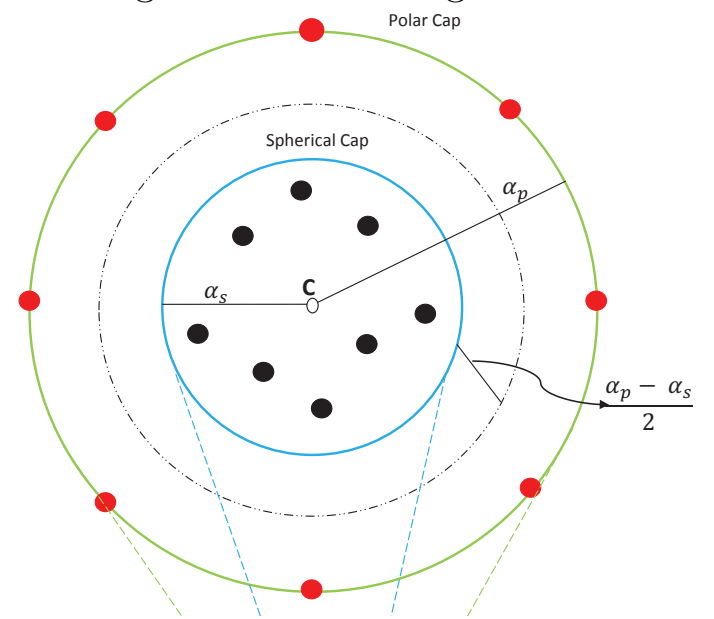

Figure 5.4: The structure of the double-cap differential codebook based on polar/spherical caps.

evenly distributed on both inner and outer caps i.e., $2^{B} / 2$ codewords on each cap. Although a double-cap differential codebook can also be designed with different numbers of codewords in two caps, we restrict our study to the equal distribution of codewords. The feedback procedure is explained in Section 5.4. Following the second feedback interval, the double-cap differential codebook radii follow the blind adaptive scaling technique discussed in the next section.

\subsubsection{Blind Adaptive Scaling}

In this section, we explain the concept of blind adaptive scaling method for the double-cap differential codebook. The advantage of having two different radii in the codebook is that the codebook can be scaled up or scaled down depending on the previously selected codeword without any additional feedback information. In order to track the channel over time, we need to maintain a distance between inner and outer caps. Therefore, we shrink and expand both the caps by $\frac{\alpha_{p}-\alpha_{s}}{2}$. The double-cap differential codebook expansion and contraction is described below.

- If the previous selected codeword at time $t$ is from the outer polar cap then expand the double-cap differential codebook for the next feedback such that $\alpha_{p}[t+1]=\alpha_{p}[t]+\left(\alpha_{p}[t]-\alpha_{s}[t]\right) / 2$ and $\alpha_{s}[t+1]=\alpha_{s}[t]+\left(\alpha_{p}[t]-\alpha_{s}[t]\right) / 2$.

- If the previous selected codeword is from the inner spherical cap then contract the double-cap differential codebook such that $\alpha_{s}[t+1]=\alpha_{s}[t]-$ $\left(\alpha_{p}[t]-\alpha_{s}[t]\right) / 2$ and $\alpha_{p}[t+1]=\alpha_{p}[t]-\left(\alpha_{p}[t]-\alpha_{s}[t]\right) / 2$. 
This method does not require the BS to have any information about the statistics of spatial and temporal correlation in the channel.

\subsection{Numerical Results and Discussion}

In this section, we present simulation results and evaluate the performance of the MU MISO system with differential codebooks. We assume that $n_{t}=4$ and $K=4$. We assume that the feedback link is lossless with zero delay. The carrier frequency is $2.5 \mathrm{GHz}$. It is also assumed that perfect CQI is available at the BS. Typically, the feedback interval is a fraction of the coherence time [26], however in this thesis, the feedback interval is set to $5 \mathrm{~ms}[46,48,103]$ which is the most common feedback interval used in standard contributions. We evaluate the performance of the MU MISO system by using ZF and SLNR precoding schemes at the BS discussed in 3.3.1 and 3.3.4, respectively.

We begin by comparing various differential codebooks discussed in this chapter using FOGM (2.13) and WINNER II (2.16) channel models. Then we present and discuss the performance of each differential codebook separately.

\subsubsection{Comparison of Differential Codebooks}

Here, we present the comparison of the spherical-cap differential codebook with Adaptive Scaling Techniques I and II and the double-cap differential codebook. The comparison is performed with respect to the average spectral efficiency against time, where time is represented in multiples of the feedback interval. The BS is equipped with $n_{t}=4$ antennas in a ULA setting with $0.5 \lambda_{0}$ spacing among neighboring antennas. The scenario considered is UMa with NLOS propagation. The base codebook is a 4 bit RVQ codebook. In Fig. 5.5, the comparison is shown at SNR, $\rho=10 \mathrm{~dB}$ with users having velocity $v=1 \mathrm{~km} / \mathrm{h}$ and $B=4$ bit feedback rate.

It is seen that the SLNR precoding scheme outperforms the ZF precoding scheme in general with differential codebooks. Also, the Adaptive Scaling Technique II with spherical-cap differential codebook gives superior average spectral efficiency compared to the spherical-cap with Adaptive Scaling Technique I and double-cap differential codebooks. However, it is important to mention that the Adaptive Scaling Technique II requires spatial/temporal correlation to be available at the BS, whereas this information is not needed in the double-cap differential codebook. Therefore, there is a trade-off between the spectral efficiency gain and the feedback overhead. On the other hand, the spherical-cap with Adaptive Scaling Technique I requires a reset to the base codebook after some multiples of the feedback interval (here, 9). In the adaptive Scaling Technique I, the frequent reset of the feedback process degrades the spectral 


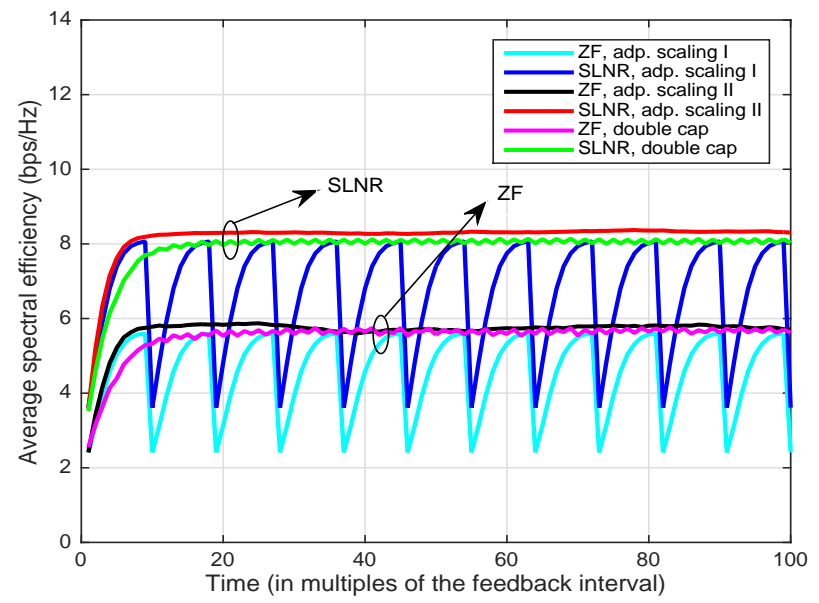

Figure 5.5: Sum rate versus time at $\rho=10 \mathrm{~dB}$ with $v=1 \mathrm{~km} / \mathrm{h}$ and 4 bit feedback in the FOGM channel.

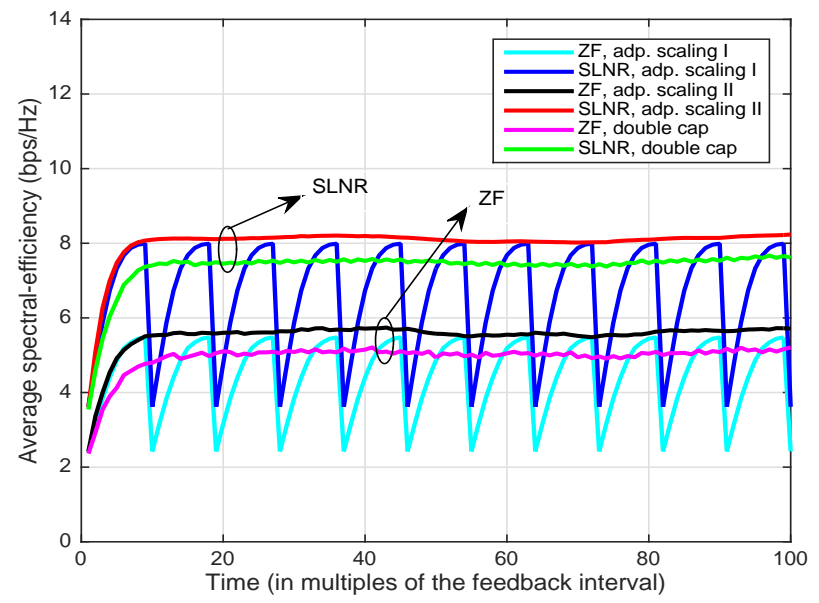

Figure 5.6: Sum rate versus time at $\rho=10 \mathrm{~dB}$ with $v=1 \mathrm{~km} / \mathrm{h}$ and 4 bit feedback in the WINNER II channel.

efficiency performance over the transmission period.

In Fig. 5.6, the same trend (as in Fig. 5.5) is noticed for the WINNER II channel. Both WINNER II channels and FOGM channels have almost similar performance given that the user velocity is same, so we only consider the FOGM channel model for the rest of the simulation results.

\subsubsection{Differential Codebooks Versus User Velocity}

In this subsection, we present the performance of differential codebooks against different user velocities. In Fig. 5.7, the average spectral efficiency is plotted against time for velocities $v=3 \mathrm{~km} / \mathrm{h}, 5 \mathrm{~km} / \mathrm{h}, 7 \mathrm{~km} / \mathrm{h}$ and $10 \mathrm{~km} / \mathrm{h}$.

We note that as the user velocity increases, the Adaptive Scaling Technique 


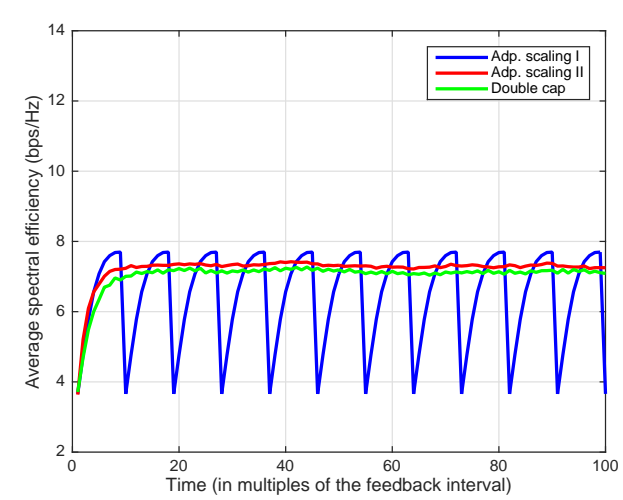

(a) $v=3 \mathrm{~km} / \mathrm{h}$

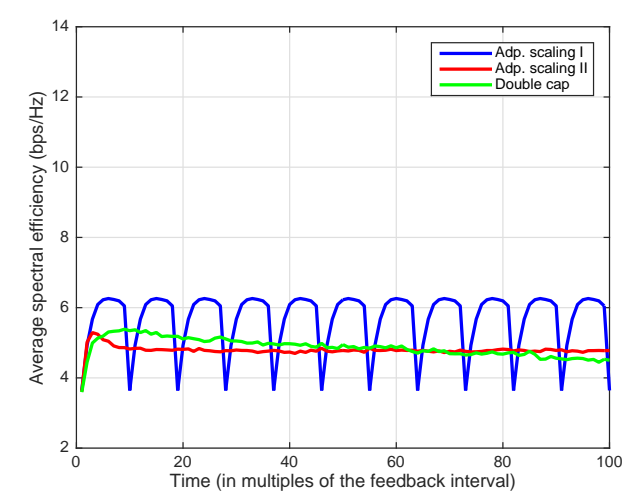

(c) $v=7 \mathrm{~km} / \mathrm{h}$

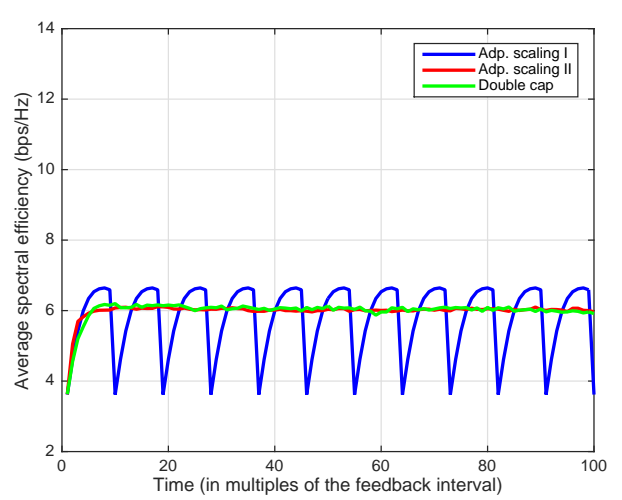

(b) $v=5 \mathrm{~km} / \mathrm{h}$

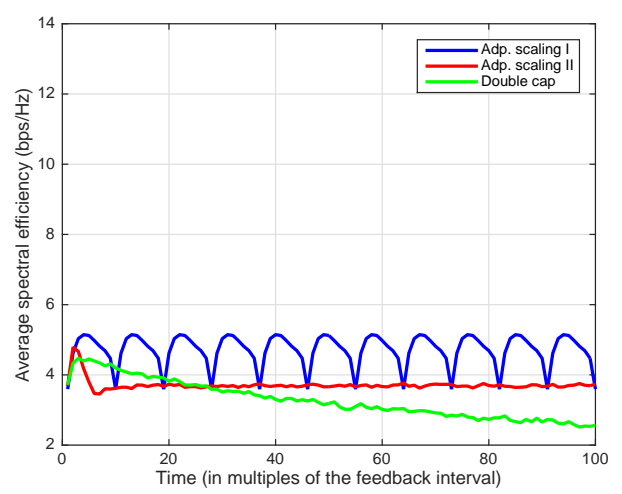

(d) $v=10 \mathrm{~km} / \mathrm{h}$

Figure 5.7: Average spectral efficiency vs. time in a temporally correlated channel at $\rho=10 \mathrm{~dB}$ for various user velocities.

II and double-cap scaling are not able to track the channel as successfully as the Adaptive Scaling Technique I. Although, the average spectral efficiency degrades as the user velocity increases, it does not degrade significantly with the Adaptive Technique I, unlike the other two methods. This is due to the reason that the Adaptive Scaling Technique I relies on the periodic reset after few feedback intervals. The double-cap codebook is capable of scaling the cap radius up and down over time, however, it yields poor performance at $v=10 \mathrm{~km} / \mathrm{h}$, indicating a failure in tracking the channel at higher velocities.

The Adaptive Scaling Technique II also show a poor average spectral efficiency performance than the Adaptive Scaling Technique I at $v=10 \mathrm{~km} / \mathrm{h}$, however unlike the double-cap, the performance after few feedback intervals matches the performance of the base codebook (here, 4 bit RVQ codebook).

\subsubsection{Estimation Errors}

Results presented so far assume perfect channel estimation at the user, but in practical systems the estimated channel at the user contains estimation errors. 


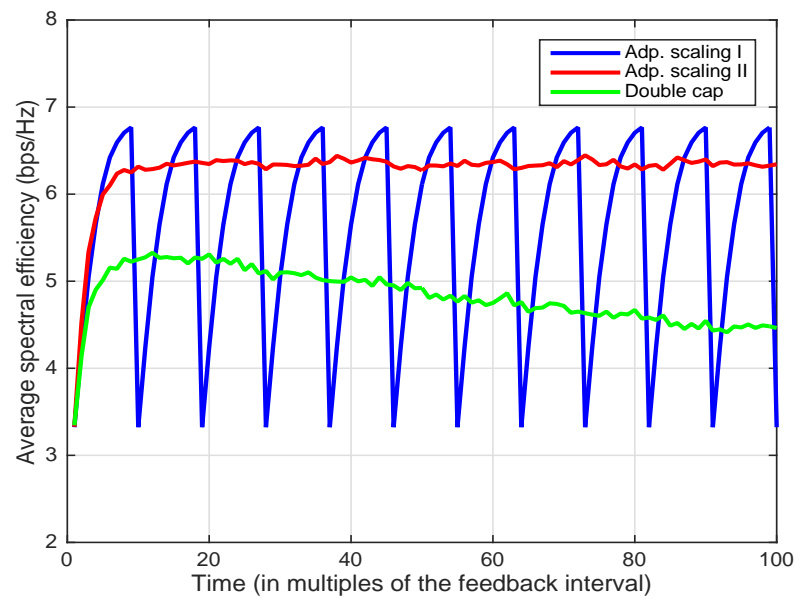

Figure 5.8: Average spectral efficiency vs. time in a temporally correlated channel at $\rho=10 \mathrm{~dB}$ for $v=3 \mathrm{~km} / \mathrm{h}$ and $\chi=0.95$.

We model the estimated channel at time $t$ as

$$
\ddot{\mathbf{h}}[t]=\chi \overline{\mathbf{h}}[t]+\sqrt{1-\chi^{2}} \mathbf{y}[t]
$$

where $\chi$ is the estimation error coefficient with $\chi=1$ yielding no estimation error and $\chi=0$ resulting in a maximum estimation error. $\mathbf{y}[t]$ denotes the innovation process with its entries distributed according to $\mathcal{C N}(0,1)$.

In Fig. 5.8, we plot the average spectral efficiency (at $\rho=10 \mathrm{~dB}$ ) against time for temporally correlated channels $(v=3 \mathrm{~km} / \mathrm{h})$ with estimation errors. We use $\chi=0.95$ which can be assumed as a small estimation error. We note that the performance of differential codebooks suffers even with the small estimation error at the user. The performance of the Adaptive Scaling Techniques I and II degrades decently, but the performance of the double-cap decreases rapidly over time.

\subsubsection{Performance of Spherical-Cap Differential Codebooks}

In this subsection, the performance of the spherical-cap differential codebook is evaluated using fixed scaling, Adaptive Scaling Technique I and Adaptive Scaling Technique II.

\section{Adaptive Scaling Technique I Results}

For the Adaptive Scaling Technique I, the differential codebook reset time is 9 feedback intervals (i.e., $45 \mathrm{~ms}$ ).

We first present the results for the temporally correlated channel, $\tilde{\mathbf{h}}$, generated using the model given in (2.13) with no spatial correlation. The base codebook that each user uses at time $t=0$ is a 4 bit RVQ codebook. We 
compare the proposed differential codebook performance with the polar cap differential codebook with adaptive scaling technique [46]. We evaluate the performance of the MU MISO system with both fixed scaling, $\alpha$, and Adaptive Scaling Technique I, $\tilde{\alpha}$. The fixed scaling parameter, $\alpha$, is the same as that used in [46] for different user speeds. In Figs. 5.9(a) and 5.9(b), the average spectral efficiency versus time results are shown for $v=1 \mathrm{~km} / \mathrm{h}$ with ZF and SLNR precoding schemes, respectively. The spherical-cap differential codebook outperforms the polar-cap codebook with both fixed and Adaptive Scaling Technique I. This is due to the fact that in the spherical-cap differential codebook, unlike [46], the codewords are evenly distributed inside the cap, and this helps in further reducing quantization errors, once the codebook starts following the channel. Also, the SLNR precoding scheme yields higher average spectral efficiency compared to the ZF precoding scheme, as the SLNR scheme not only suppresses the interference but also maximizes the SNR.

In Fig. 5.10(a) and 5.10(b), the average spectral efficiency is shown for the user speed $v=3 \mathrm{~km} / \mathrm{h}$ with ZF and SLNR precoding schemes, respectively. The spectral efficiency loss is higher when the speed is $v=3 \mathrm{~km} / \mathrm{h}$ compared to the $v=1 \mathrm{~km} / \mathrm{h}$ case. As the user speed increases, the quantization errors also become large, which is the reason for the spectral efficiency loss.

Figure 5.11 shows the average minimum chordal distance given by (5.26). Small values of the scaling parameter, $\alpha$, can be seen to yield a small minimum chordal distance when the user speed is slow. As the user speed increases, large scaling parameters perform better by yielding small average minimum chordal distance. It is also evident that a scaling parameter of 0.1 is too small and does not help the differential codebook to track the temporally correlated channel. A suitable choice of $\alpha$ for different speeds can be obtained from Fig. 5.11, and is also given in Table 5.1.

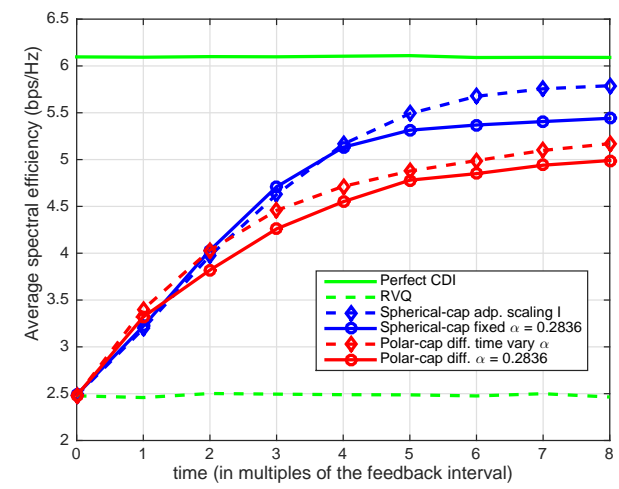

(a) $\mathrm{ZF}$

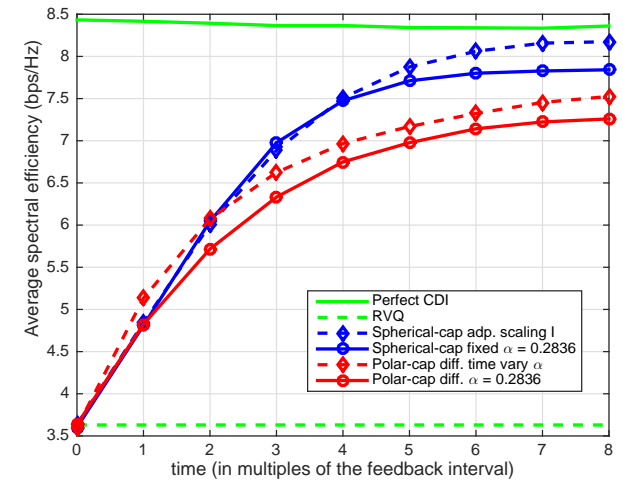

(b) SLNR

Figure 5.9: Average spectral efficiency vs. time in a temporally correlated channel at $\rho=10 \mathrm{~dB}$ with $v=1 \mathrm{~km} / \mathrm{h}(\epsilon=0.9987)$. 


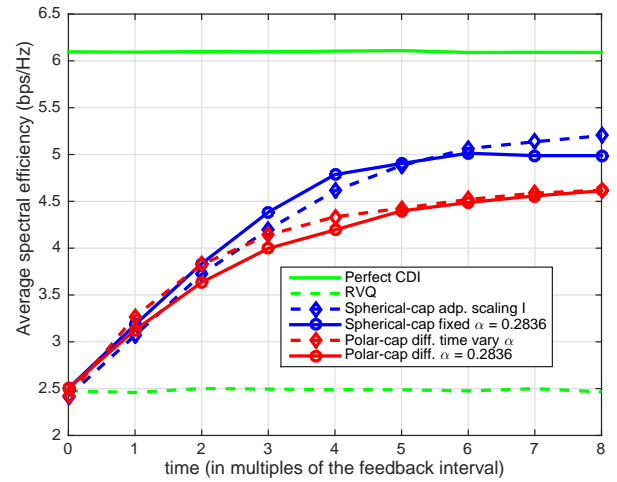

(a) $\mathrm{ZF}$

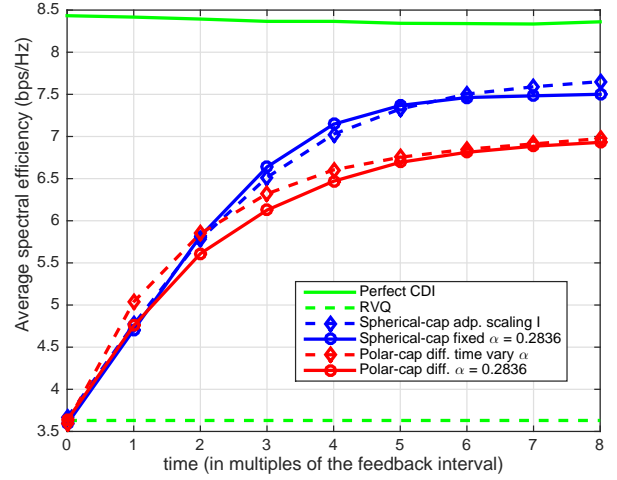

(b) SLNR

Figure 5.10: Average spectral efficiency vs. time in a temporally correlated channel at $\rho=10 \mathrm{~dB}$ with $v=3 \mathrm{~km} / \mathrm{h}(\epsilon=0.9881)$.

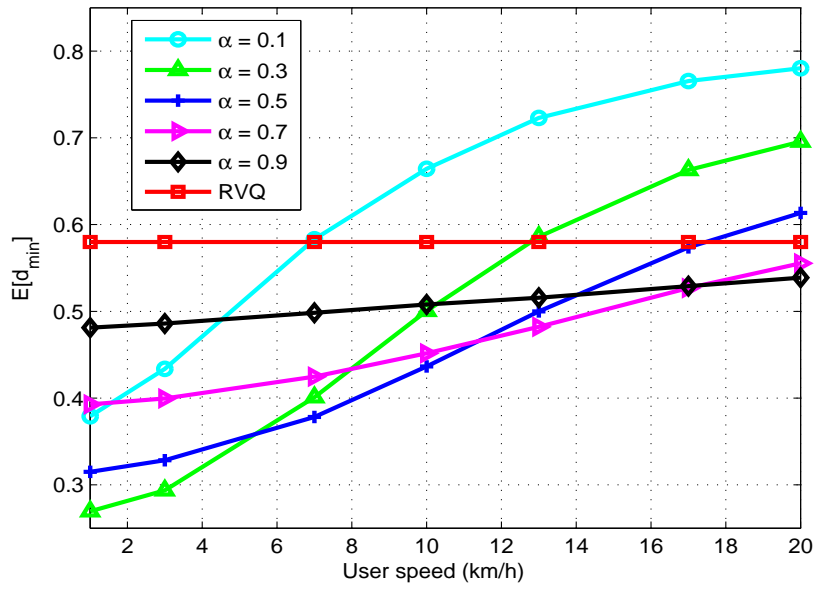

Figure 5.11: Average minimum chordal distance vs. user speed in temporally correlated channels.

Table 5.1: Scaling Parameters in Temporally Correlated Channels

\begin{tabular}{|c||c|c|c|c|}
\hline User speed $v(\mathrm{~km} / \mathrm{h})$ & $2-6$ & $6-12$ & $12-17$ & $17-20$ \\
\hline \hline Scaling parameter $\alpha$ & 0.3 & 0.5 & 0.7 & 0.9 \\
\hline
\end{tabular}

We evaluate the performance of the spherical-cap differential codebook with fixed scaling, $\alpha$, and compare it to the polar-cap differential codebook. The spatially and temporally correlated channel is modeled by (2.14). We use the 4 bit 3GPP LTE rel.8 codebook as a base codebook because the DFT codewords in the LTE codebook improve the performance in the spatially correlated channel [46]. As scheduling the users is not the part of this work, during simulation we discard the case when more than one user selects the same codeword from the LTE codebook. Reporting the same codeword affects the channel inversion process of the ZF precoding scheme. 
In Fig. 5.12, the average spectral efficiency is evaluated when $v=3 \mathrm{~km} / \mathrm{h}$ and $z_{\mathrm{t}}=0.5$. The performance is analyzed with two fixed scaling parameters given in [46]; 0.1142 and 0.2836 . For low spatial correlation, the channel approaches i.i.d., and thus the scaling parameter $\alpha$ needs to be increased. This is the reason that the performance gain with $\alpha=0.2836$ is higher than with $\alpha=0.1142$.

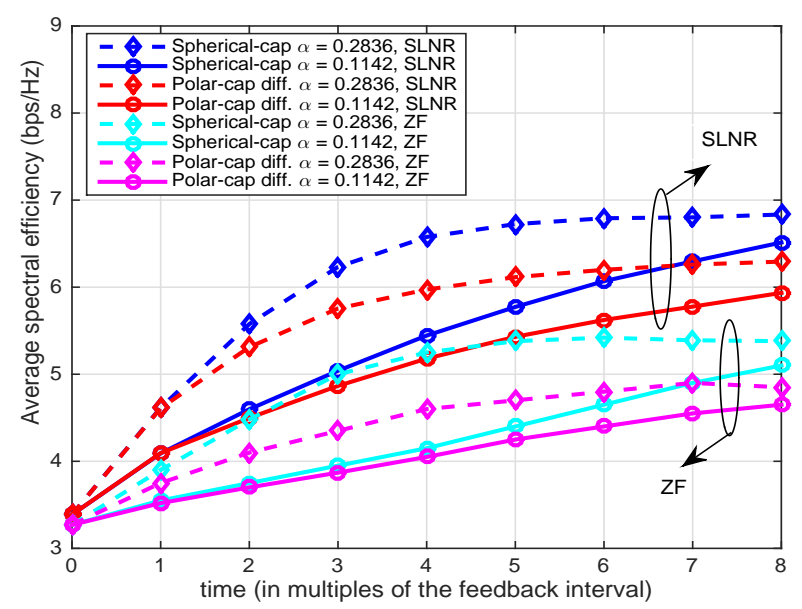

Figure 5.12: Average spectral efficiency vs. time in a spatially/temporally correlated channel at $\rho=10 \mathrm{~dB}$ with $v=3 \mathrm{~km} / \mathrm{h}(\epsilon=0.9881)$ and $z_{\mathrm{t}}=0.5$.

It is evident from the simulation results that the spherical-cap differential codebook with fixed scaling or Adaptive Scaling Technique I, successfully exploits the correlation, both spatial and temporal, present in the channel and helps the system to achieve a better average spectral efficiency.

\section{Adaptive Scaling Technique II Results}

Now we present the simulation results for the Adaptive Scaling Technique II with the spherical-cap differential codebook. Again, we use both ZF and SLNR precoding schemes to evaluate the MU MISO system. The Adaptive Scaling Technique II discussed in Section 5.7.2 consider both temporal and spatial correlations, therefore, we use the FOGM (2.14) channel model to evaluate the performance of the Adaptive Scaling Technique II. For the FOGM channel model, the value of $z_{\mathrm{t}}$ is set to 0.5 . The base codebook is a 4 bit RVQ codebook.

Figures 5.13(a) and 5.13(b) show the average spectral efficiency versus time with $v=1 \mathrm{~km} / \mathrm{h}$ and $v=3 \mathrm{~km} / \mathrm{h}$ at $\rho=10 \mathrm{~dB}$ for ZF and SLNR precoding schemes, respectively. The average spectral efficiency with the spherical-cap differential codebook with Adaptive Scaling Technique II is higher than the RVQ codebook and the polar-cap differential codebook. The average spectral efficiency does not degrade over time with the the Adaptive Scaling Technique II and remains stable over time. When the speed increases, the average spectral efficiency decreases, as in the case of $v=3 \mathrm{~km} / \mathrm{h}$, implying that quantization 


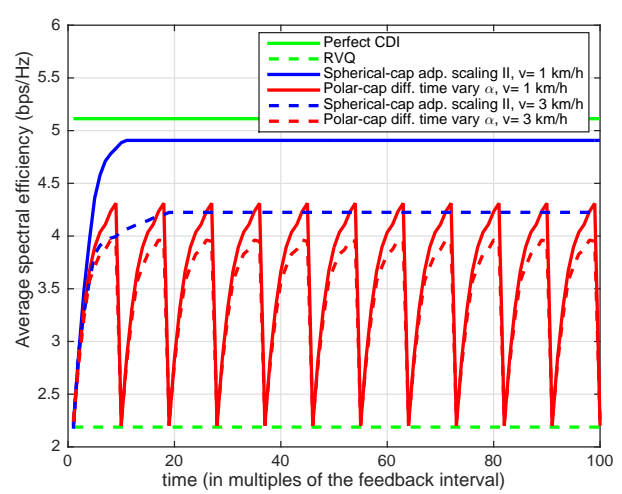

(a) ZF

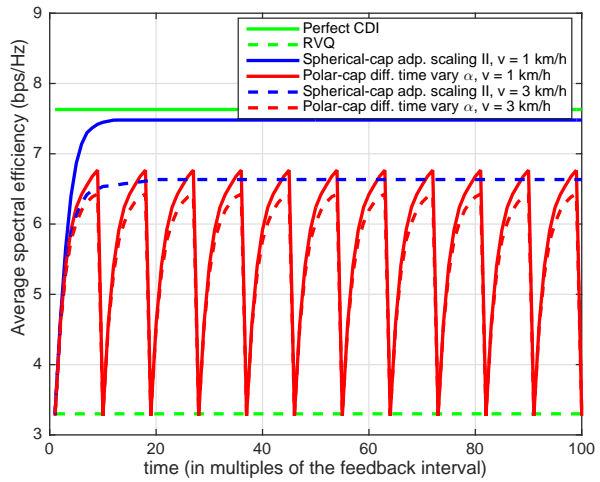

(b) SLNR

Figure 5.13: Average spectral efficiency vs time at $\rho=10 \mathrm{~dB}$ for $z_{t}=0.5$ with $v=1 \mathrm{~km} / \mathrm{h}$ and $v=3 \mathrm{~km} / \mathrm{h}$.

errors are large and the spherical-cap differential codebook uses a large scaling parameter in order to track the varying channel.

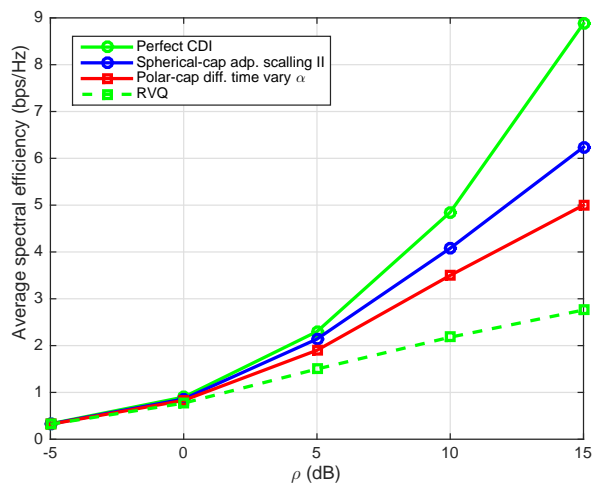

(a) ZF

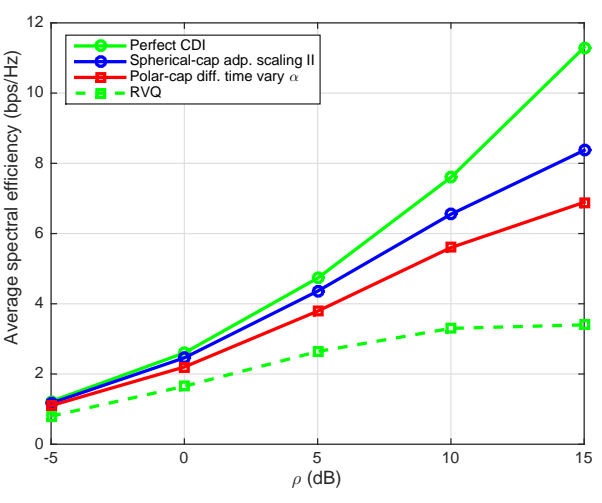

(b) SLNR

Figure 5.14: Average spectral efficiency vs. $\rho$ for different user speeds with $v=3$ $\mathrm{km} / \mathrm{h}$.

Figures 5.14(a) and 5.14(b) show average spectral efficiency against the range of $\rho$ values for ZF and SLNR precoding schemes, respectively. The performance of the spherical-cap differential codebook with Adaptive Scaling Technique II is superior than the polar-cap differential codebook because it achieves temporal stability in the spectral efficiency and has a better codeword arrangement inside the codebook.

In Fig. 5.15, the CDF of the SINR is plotted for one of four users when the user speed is $v=1 \mathrm{~km} / \mathrm{h}$. We compare three cases: perfect CDI, the sphericalcap differential codebook with Adaptive Scaling Technique II, and the RVQ codebook for both ZF and SLNR precoding schemes. The SLNR precoding outperforms the ZF precoding scheme and with the Adaptive Scaling Technique 


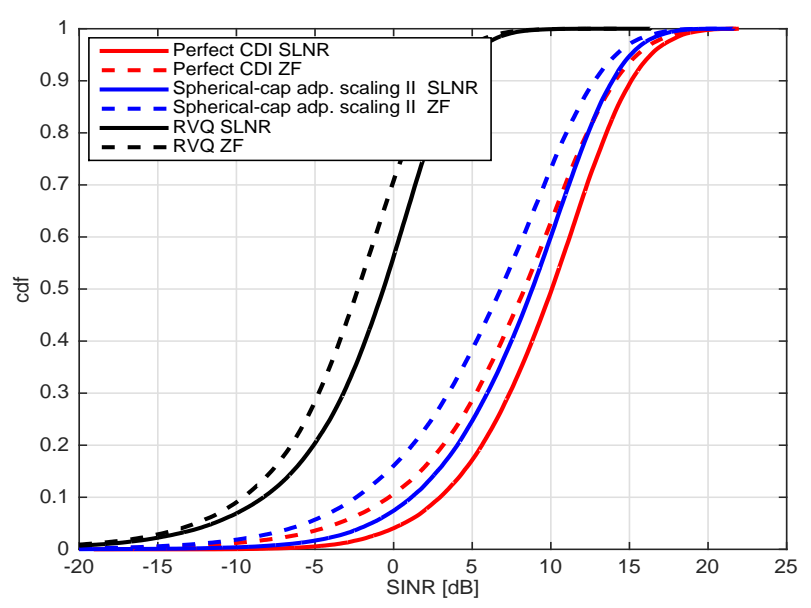

Figure 5.15: SINR CDF for the user at $\rho=16 \mathrm{~dB}$ with $v=1 \mathrm{~km} / \mathrm{h}(\epsilon=0.9987)$.

II it is even better than the perfect CDI ZF case at $v=1 \mathrm{~km} / \mathrm{h}$.

\subsubsection{Performance of Double-Cap Differential Codebook}

In this subsection, we evaluate the performance of the double-cap differential codebook discussed in Section 5.8 over temporally correlated MISO channels. The MU MISO channel is modeled using the FOGM channel model, $\tilde{\mathbf{h}}(2.13)$. The base codebook used is the 4 bit RVQ codebook.

Figure 5.16 shows the average spectral efficiency versus time results for the double-cap differential codebook at SNR $=10 \mathrm{~dB}$ with ZF and SLNR precoding schemes. The speed of the users is $v=1 \mathrm{~km} / \mathrm{h}$. The codewords are evenly distributed on both caps (i.e., 8 on the inner and 8 on the outer cap) in the double-cap differential codebook. The average spectral efficiency achieved by

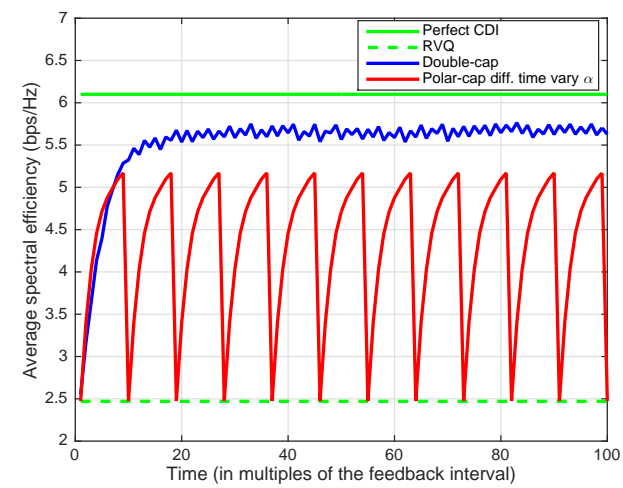

(a) ZF

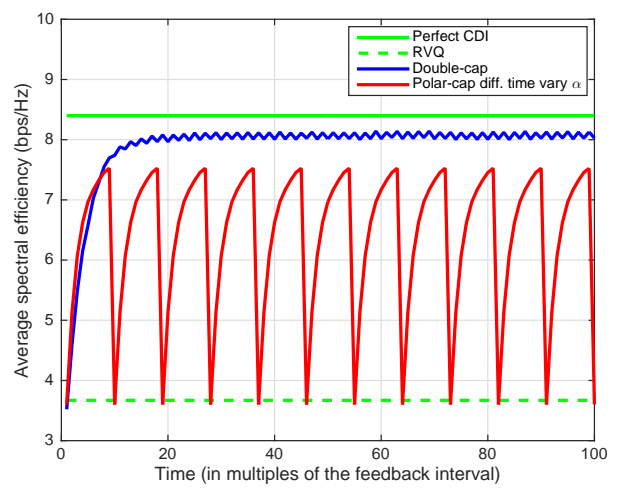

(b) SLNR

Figure 5.16: Average spectral efficiency versus time at $\rho=10 \mathrm{~dB}$ with $v=1$ $\mathrm{km} / \mathrm{h}$ and 4 bit feedback. 


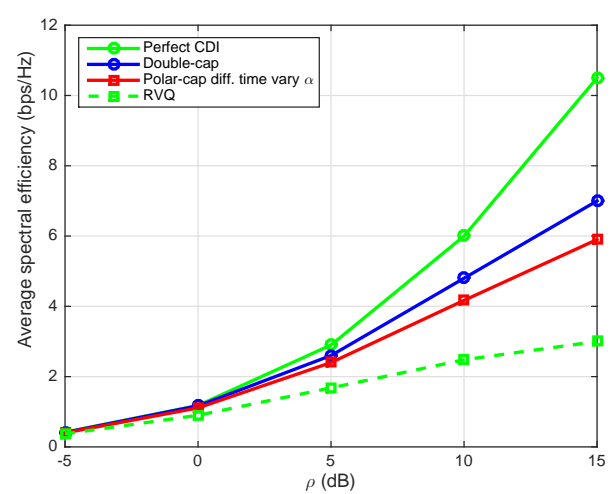

(a) $\mathrm{ZF}$

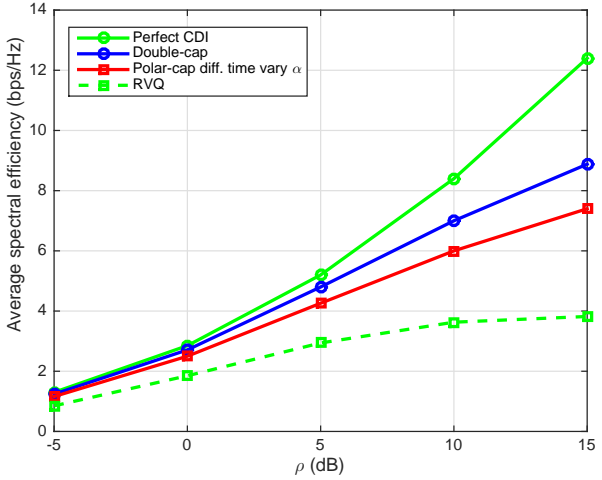

(b) SLNR

Figure 5.17: Average spectral efficiency against $\rho$ values with $v=3 \mathrm{~km} / \mathrm{h}$ and 4 bit feedback (100 feedback intervals).

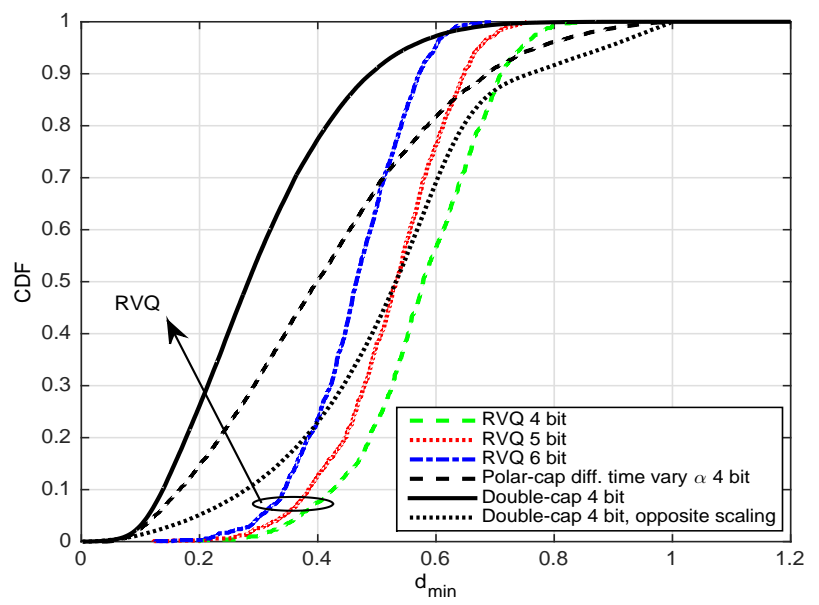

Figure 5.18: Quantization errors CDF at $v=3 \mathrm{~km} / \mathrm{h}$ (100 feedback intervals).

the double-cap differential codebook is significant and outperforms the polar cap differential codebook. The codebook reset time for the polar cap differential codebook is set to 8 feedback intervals [46]. It is important to note here that the double-cap differential codebook is using blind adaptive scaling, whereas in the case of the polar cap differential codebook, the adaptive scaling assumes that the BS has perfect temporal correlation information. The blind adaptive scaling technique for the double-cap is capable of scaling up and scaling down the cap radii, and it therefore works well for longer transmission times. Average spectral efficiency is plotted against different SNR values in Fig. 5.17 for $v=3 \mathrm{~km} / \mathrm{h}$ for ZF and SLNR precoding schemes. The double-cap differential codebook gives superior spectral efficiency compared to the polar-cap differential codebook and the RVQ codebook with 4 bit feedback. Fig. 5.18 shows the quantization error CDF in terms of the chordal distance, given by (3.14), for the double-cap differential codebook, the polar cap differential codebook with 
4 bit and RVQ codebooks with 4, 5 and 6 bits feedback. It is seen that even with 6 bit feedback, the RVQ codebook gives larger quantization errors than the 4 bit double-cap differential codebook. Also, the double-cap differential codebook produces small quantization errors compared to the polar cap differential codebook, implying that the double-cap differential codebook tracks the channel more effectively. It is seen that opposite scaling (i.e., scaling up the differential codebook when an inner cap codeword is selected and vice versa) results in a poor quantization performance compared to other differential codebooks, thus validating the intuition behind the proposed scaling approach.

\subsection{Summary}

In this chapter, we have discussed two differential codebook structures: single spherical-cap and double cap. For single spherical-cap differential codebook, we discussed two adaptive scaling techniques: Adaptive Scaling Technique I and Adaptive Scaling Technique II. Adaptive Scaling Technique I does not require any additional feedback information but needs to be reset periodically, hence loosing the performance gain regularly. However, Adaptive Scaling Technique II works well for longer transmission periods without a reset. Also, Adaptive Scaling Technique II yields better performance than the Adaptive Scaling Technique I in the cost of additional feedback information about spatial and temporal correlation. Therefore, there is a trade off between two techniques.

Finally, the double cap differential codebook is discussed that consists of two caps: a spherical cap and a polar cap. The two cap design allows the caps radii to blindly adapt in a systematic manner without the requirement of a reset and additional feedback information. The double cap differential codebook has slightly lower performance than the spherical-cap differential codebooks, but it does not exhibit the disadvantages of the periodic reset and the additional feedback of the correlation statistics. We also note that the double-cap differential codebook does not work well for higher velocities and also show a poor performance when the channel at the receiver has estimation errors. 


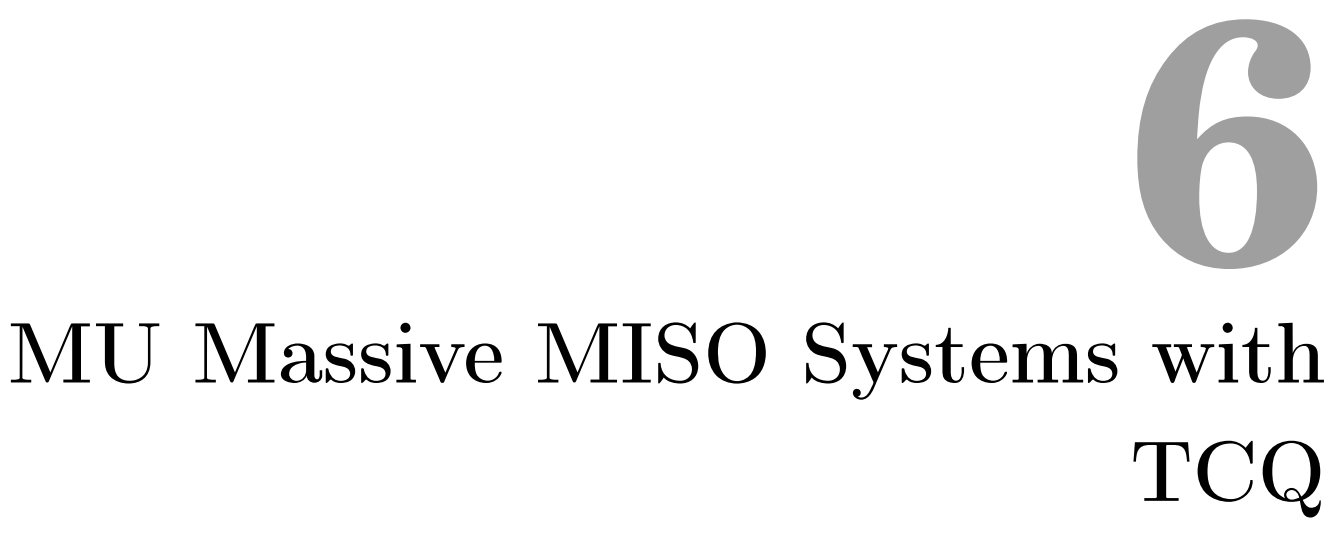

previous chapters discuss conventional multi-antenna systems where the number of antennas at the BS is small. This chapter considers limited feedback in massive MISO systems, where the BS consists of a large number of transmit antennas and simultaneously serves relatively few single antenna users. In codebook-based limited feedback systems, the requirement of the number of codewords in the codebook increases due to the large number of transmit antennas. In turn, increasing the size of the codebook results in a higher search complexity while selecting an appropriate codeword from the codebook. Therefore, codebook-based limited feedback systems are not considered practical for massive MIMO/MISO systems. In this chapter, we present differential TCQ schemes for limited feedback massive MISO FDD systems in temporally and spatially correlated channels. The TCQ schemes presented in this chapter have lower search complexity than the conventional codebook approach and are thus suitable for massive MISO systems.

\subsection{Motivation and Related Work}

Recently, massive MISO has been widely investigated [47,48] in FDD based limited feedback massive MIMO systems. Most of the research on massive MIMO considers TDD operations, but the performance of massive MISO based TDD systems is limited due to practical issues such as pilot contamination [34], RF calibration errors [115] and no downlink CSI training at the receiver [116]. In fact, most of the existing cellular systems use FDD operation. 
For a spatially correlated channel, a compressive sensing-based feedback scheme is proposed in [117] with FDD operation, where the feedback contents are dynamically configured depending on channel conditions. Recently, open-loop and closed-loop training techniques have been developed in [116] for massive MISO FDD systems, where long-term channel statistics and previously received training signals are used to increase the performance of channel estimation at the user. Moreover, with a small amount of feedback overhead, it is shown in [116] that the closed-loop training scheme reduces the downlink training overhead. Recently, trellis based channel quantization methods have drawn much attention in limited feedback massive MISO FDD systems due to their low complexity compared to conventional codebook approaches.

In TCQ based limited feedback systems, the channel is quantized by the user using the concept of a trellis with a source constellation and the Viterbi algorithm. The input bit sequence of the selected trellis path is fed back to the $\mathrm{BS}$, where the BS uses a convolutional coder to regenerate the quantized channel and computes the beamforming vectors. In conventional channel coding, the Viterbi algorithm runs over the trellis stages where each stage represents time, in limited feedback MISO systems with TCQ, each trellis stage corresponds to a particular BS antenna element.

One way of selecting the trellis path is by using brute-force maximumlikelihood (ML) optimization that searches for the best path (most likely sequence) from the set of all the trellis paths (also known as trellis codewords). However, due to the exhaustive search, it is not practical to consider bruteforce ML, therefore the Viterbi algorithm is preferred as it reduces the search complexity by relying on the surviving trellis paths only.

A trellis based channel quantization scheme is proposed in [47], for SU multicell MISO systems with large numbers of BS antennas. A noncoherent TCQ (NTCQ) approach for a massive MISO system is proposed in [48], where a bank of coherent detectors is implemented to realize near optimal noncoherent detection. Here, TCQ with Ungerboeck's trellis is used to quantize the CDI for a SU massive MISO system. By adopting Ungerboeck's TCM structures, the TCQ scheme uses source constellations such as QPSK, 8PSK or 16QAM to quantize channel entries. A convolutional code is implemented at the BS to reconstruct the quantized channel. Three different limited feedback schemes are proposed in [48] for three different channel models; an i.i.d. Rayleigh fading channel, a temporally correlated channel and a spatially correlated channel. The drawback of differential NTCQ method in [48] is that the quantization process requires additional feedback of optimization parameters, thus increasing the feedback overhead. On the other hand, the adaptive TCQ method proposed in [48] for spatially correlated channels requires knowledge of transmit correlation at the transmitter. 
In this chapter, we adopt the TCQ framework to quantize the MISO channel at each user for temporally correlated channels. Exploiting the temporal correlation in the channel, we design a differential TCQ in [118] scheme that transforms the source constellation at each stage in a trellis, such that it is centered around the previously selected constellation point, for the next feedback instance. We use 2D translation and scaling schemes to transform the source constellation. We derive a scaling parameter for the source constellation that is a function of the amount of temporal correlation and the number of transmit antennas.

We also discuss a TCQ based limited feedback scheme for spatially correlated channels in [118] where we quantize correlated channel directly at the receiver. Hence, unlike [48], the proposed scheme does not require knowledge of the transmit correlation matrix at the transmitter. For spatially correlated channels, we consider two antenna topologies: uniform linear array (ULA) and uniform rectangular array (URA).

\subsection{System Description}

Consider a single-cell MU MISO system with $n_{t}$ transmit antennas at the BS. The BS serves $K$ single antenna users simultaneously using a suitable precoding technique, where $K<n_{t}$ with a constant ratio $q=n_{t} / K$. On the downlink, the received signal for the $k^{\text {th }}$ user can be written as

$$
y_{k}=\sqrt{\frac{\rho}{K}} \tilde{\mathbf{h}}_{k} \mathbf{x}+n_{k}, \quad k=1, \ldots, K
$$

where $\tilde{\mathbf{h}}_{k}$ denotes the temporally correlated MISO channel modeled by FOGM process given in (2.13). $n_{k}$ is the noise assumed to be i.i.d. with $n_{k} \sim \mathcal{C N}(0,1)$ $\forall k$ and $\rho$ is the SNR. We assume uniform power allocation among $K$ users. The transmitted signal is given by $\mathbf{x}=\sum_{k=1}^{K} \mathbf{w}_{k} s_{k}$, where $s_{k}$ and $\mathbf{w}_{k}$ are the data symbol and $n_{t} \times 1$ unit-norm precoding vector for the $k^{\text {th }}$ user, respectively.

We use TCQ schemes (to be discussed later) to quantize CDI vectors, $\tilde{\mathbf{u}}_{k}=$ $\frac{\tilde{\mathbf{h}}_{k}}{\left\|\hat{\mathbf{h}}_{k}\right\|}$, for temporally correlated channels $(2.13)$, and $\hat{\mathbf{u}}_{k}=\frac{\hat{\mathbf{h}}_{k}}{\left\|\hat{\mathbf{h}}_{k}\right\|}$, for spatially correlated channels (2.11). We denote the "quantized" CDI for the $k^{\text {th }}$ user by $\tilde{\mathbf{q}}_{k}$ and $\hat{\mathbf{q}}_{k}$, for temporally and spatially correlated channels, respectively.

\subsection{Expected SINR with MF Precoding}

In this section, we derive approximations of the expected SINR with both perfect CDI and RVQ codebook-based CDI, with MF precoding and large $n_{t}$. The SINR approximations derived here are used in Section 6.4 to compute the number of 
bits required to obtain a mean SINR performance with RVQ CDI that is $z \mathrm{~dB}$ below the SINR with perfect CDI. The downlink received signal in (6.1) can also be written as

$$
y_{k}=\underbrace{\sqrt{\frac{\rho}{K}}\left(\tilde{\mathbf{h}}_{k} \mathbf{w}_{k}\right) s_{k}}_{\text {desired signal }}+\underbrace{\sum_{j \neq k} \sqrt{\frac{\rho}{K}}\left(\tilde{\mathbf{h}}_{k} \mathbf{w}_{j}\right) s_{j}}_{\text {interference }}+\underbrace{n_{k}}_{\text {noise }} .
$$

Using the SINR in (3.7), the expected SINR can be written as

$$
\mathbb{E}\left[\mathrm{SINR}_{k}\right]=\mathbb{E}\left[\frac{\frac{\rho}{K}\left|\tilde{\mathbf{h}}_{k} \mathbf{w}_{k}\right|^{2}}{\frac{\rho}{K} \sum_{j \neq i}^{K}\left|\tilde{\mathbf{h}}_{k} \mathbf{w}_{j}\right|^{2}+1}\right] .
$$

Using the approach given in [119] to evaluate the expectation over the numerator and the denominator, where both are functions of the same random variables, and $n_{t} \rightarrow \infty$, we can write $(6.3)$ as

$$
\mathbb{E}\left[\mathrm{SINR}_{k}\right] \approx \frac{\frac{\rho}{K} \mathbb{E}\left[\left|\tilde{\mathbf{h}}_{k} \mathbf{w}_{k}\right|^{2}\right]}{\frac{\rho}{K} \sum_{j \neq i}^{K} \mathbb{E}\left[\left|\tilde{\mathbf{h}}_{k} \mathbf{w}_{j}\right|^{2}\right]+1} .
$$

\subsubsection{Perfect CDI}

For MF precoding with perfect CDI, we have $\mathbf{w}_{k}=\tilde{\mathbf{u}}_{k}^{H}$ and the expected SINR approximation for the $k^{\text {th }}$ user using, (6.4), is written as

$$
\begin{aligned}
\mathbb{E}\left[\operatorname{SINR}_{k}\right] & \approx \frac{\frac{\rho}{K} \mathbb{E}\left[\left|\tilde{\mathbf{h}}_{k} \mathbf{w}_{k}\right|^{2}\right]}{\frac{\rho}{K} \sum_{j \neq i}^{K} \mathbb{E}\left[\left|\tilde{\mathbf{h}}_{k} \mathbf{w}_{j}\right|^{2}\right]+1} \\
& \stackrel{(a)}{=} \frac{\frac{\rho}{K} \mathbb{E}\left[\left\|\tilde{\mathbf{h}}_{k}\right\|^{2}\right] \mathbb{E}\left[\left|\tilde{\mathbf{u}}_{k} \tilde{\mathbf{u}}_{k}^{H}\right|^{2}\right]}{\frac{\rho}{K} \mathbb{E}\left[\left\|\tilde{\mathbf{h}}_{k}\right\|^{2}\right] \sum_{j \neq k}^{K} \mathbb{E}\left[\left|\tilde{\mathbf{u}}_{k} \tilde{\mathbf{u}}_{j}^{H}\right|^{2}\right]+1},
\end{aligned}
$$

where (a) is due to the fact that amplitude and direction of $\tilde{\mathbf{h}}_{k}$ are independent [102]. In (6.6), $\mathbb{E}\left[\left\|\tilde{\mathbf{h}}_{k}\right\|^{2}\right]=n_{t}$ and $\mathbb{E}\left[\left|\tilde{\mathbf{u}}_{k} \tilde{\mathbf{u}}_{k}^{H}\right|^{2}\right]=1$. We note that $\mathbb{E}\left[\left|\tilde{\mathbf{u}}_{k} \tilde{\mathbf{u}}_{j}^{H}\right|^{2}\right]=$ $\frac{1}{n_{t}}$, as $\tilde{\mathbf{u}}_{k}$ and $\tilde{\mathbf{u}}_{j}$ are independent unit norm vectors. Therefore, the expected SINR approximation becomes

$$
\mathbb{E}\left[\mathrm{SINR}_{k}\right] \approx \frac{\rho q}{\frac{\rho(K-1)}{K}+1}
$$


which at high SNR $(\rho \rightarrow \infty)$, can be written as

$$
\lim _{\rho \rightarrow \infty} \mathbb{E}\left[\mathrm{SINR}_{k}\right] \approx \frac{n_{t}}{K-1}
$$

\subsubsection{RVQ Codebook CDI}

Consider a limited feedback system where the CDI is quantized using an RVQ codebook of size $N_{c}$, thus requiring $B=\log _{2}\left(N_{c}\right)$ feedback bits per user. Let us denote the selected RVQ codeword vector of size $n_{t} \times 1$ for the $k^{\text {th }}$ user as $\tilde{\mathbf{q}}_{k}$, using (3.14). For MF precoding with RVQ limited feedback, the expected SINR approximation for the $k^{\text {th }}$ user, denoted by $\mathbb{E}\left[\widetilde{\mathrm{SINR}}_{k}\right]$, becomes

$$
\begin{aligned}
\mathbb{E}\left[\widetilde{\operatorname{SINR}}_{k}\right] & \approx \frac{\frac{\rho}{K} \mathbb{E}\left[\left|\tilde{\mathbf{h}}_{k} \tilde{\mathbf{q}}_{k}^{H}\right|^{2}\right]}{\frac{\rho}{K} \mathbb{E}\left[\sum_{j \neq k}^{K}\left|\tilde{\mathbf{h}}_{k} \tilde{\mathbf{q}}_{j}^{H}\right|^{2}\right]+1} \\
& =\frac{\frac{\rho}{K} \mathbb{E}\left[\left\|\tilde{\mathbf{h}}_{k}\right\|^{2}\right] \mathbb{E}\left[\left|\tilde{\mathbf{u}}_{k} \tilde{\mathbf{q}}_{k}^{H}\right|^{2}\right]}{\frac{\rho}{K} \mathbb{E}\left[\left\|\tilde{\mathbf{h}}_{k}\right\|^{2}\right] \sum_{j \neq k}^{K} \mathbb{E}\left[\left|\tilde{\mathbf{u}}_{k} \tilde{\mathbf{q}}_{j}^{H}\right|^{2}\right]+1} .
\end{aligned}
$$

Equation (6.10) comes from the independence between the amplitude and direction of $\tilde{\mathbf{h}}_{k}[102]$. It is shown in [22], that the expectation $\mathbb{E}\left[\left|\tilde{\mathbf{u}}_{k} \tilde{\mathbf{q}}_{k}^{H}\right|^{2}\right]$ is given by

$$
\mathbb{E}\left[\left|\tilde{\mathbf{u}}_{k} \tilde{\mathbf{q}}_{k}^{H}\right|^{2}\right]=1-N_{c} \beta\left(N_{c}, \frac{n_{t}}{n_{t}-1}\right) \triangleq 1-\mathbb{E}[\mathcal{Q}],
$$

where $\beta(\cdot, \cdot)$ denotes a beta function. In $[22]$, an upper bound on $\mathbb{E}[\mathcal{Q}]$ is given by

$$
\mathbb{E}[\mathcal{Q}] \leq 2^{\frac{-B}{n^{-1}}}
$$

Due to the independence between the unit norm vectors $\tilde{\mathbf{u}}_{k}$ and $\tilde{\mathbf{q}}_{j}$, the second expectation in the denominator of $(6.10)$, is $\mathbb{E}\left[\left|\tilde{\mathbf{u}}_{k} \tilde{\mathbf{q}}_{j}^{H}\right|^{2}\right]=\frac{1}{n_{t}}$. Also, $\mathbb{E}\left[\left\|\tilde{\mathbf{h}}_{k}\right\|^{2}\right]=n_{t}$ and thus, the expected SINR approximation for the $k^{\text {th }}$ user for the MF precoding system with RVQ codebooks can be expressed as

$$
\mathbb{E}\left[\widetilde{\mathrm{SINR}}_{k}\right] \approx \frac{\rho q(1-\mathbb{E}[\mathcal{Q}])}{\frac{\rho}{K}(K-1)+1} .
$$

At high SNR $(\rho \rightarrow \infty),(6.13)$ becomes

$$
\lim _{\rho \rightarrow \infty} \mathbb{E}\left[{\widetilde{\operatorname{SINR}_{k}}}\right] \approx \frac{n_{t}(1-\mathbb{E}[\mathcal{Q}])}{K-1}
$$




\subsection{Impracticality of RVQ Codebooks For Mas- sive MISO}

In this section, we show that codebook based limited feedback approaches are not practical in massive MISO systems. Although this is known in an intuitive sense, here we quantify the scale of the problem by computing the size of the codebook required for a particular performance target. In particular, using the results of Section 6.3, we derive the number of bits required to achieve an expected SINR performance with RVQ codebooks that suffers a $z$ dB loss compared to the expected SINR performance with perfect CDI. We also briefly discuss the search complexity of the quantization process with RVQ codebooks. Such calculations require the RVQ assumption but give a clear indication of the order of the codebook size for other more practical schemes.

The expected SINR approximations for MF precoding with both perfect CDI and RVQ codebook CDI, derived in the previous section allows us to compute the number of bits that is required to achieve an expected SINR with RVQ which is $z \mathrm{~dB}$ below the expected SINR with perfect CDI. We use (6.7) and (6.13) to obtain

$$
\frac{\mathbb{E}\left[\mathrm{SINR}_{k}\right]}{10^{\frac{z}{10}}}=\mathbb{E}\left[{\widetilde{\operatorname{SINR}_{k}}}\right]
$$

and thus

$$
\frac{\rho q}{10^{\frac{z}{10}}\left(\frac{\rho(K-1)+1}{K}\right)}=\frac{\rho q\left(1-2^{\frac{-B}{n_{t}-1}}\right)}{\frac{\rho}{K}(K-1)+1} .
$$

Solving (6.16) for the number of feedback bits required, we have

$$
B_{\text {req }}^{z}=-\left(n_{t}-1\right) \log _{2}\left(1-\frac{1}{10^{\frac{z}{10}}}\right) \text {. }
$$

We note that in MF precoding systems with RVQ codebooks, unlike ZF precoding [27], the number of feedback bits required to represent the quantized channel does not depend on $\rho$. If $n_{t}=100$ and $z=3 \mathrm{~dB}$, the number of bits required to obtain a half of the perfect CDI expected SINR performance is, $B_{\text {req }}^{z}=99$ bits i.e., a codebook of size $2^{99}=6.3383 \times 10^{29}$. Even to achieve a very low target where the signal power is equal to the interference-plus-noise power, i.e., $\mathbb{E}\left[\overline{\mathrm{SINR}}_{k}\right]=1$, the number of bits required is

$$
B_{\text {req }}=-\left(n_{t}-1\right) \log _{2}\left(1-\frac{K-1}{n_{t}}\right) .
$$


For example, for $n_{t}=100$ and $K=10$, using $(6.18)$ we have $B_{\text {req }}=13.4701 \approx$ 14, which corresponds to 16384 codebook entries per user. While the feedback overhead with large $n_{t}$ may be acceptable, the search for an appropriate codeword within the codebook is very challenging and becomes computationally infeasible as $n_{t}$ increases. The search complexity for the RVQ codebook quantization, given by $O\left(n_{t} 2^{B n_{t}}\right)$ [48], grows exponentially with large $n_{t}$. The memory requirement also increases at the user with large codebook sizes. Therefore, codebook-based limited feedback schemes are infeasible for massive multiantenna systems. This serves as a motivation to seek a non-codebook approach for limited feedback MISO systems. Therefore, we develop two limited feedback schemes for temporally and spatially correlated channels in Section 6.6 and Section 6.7, respectively, that are based on the (non-codebook) TCQ scheme.

\subsection{Limited Feedback with TCQ}

In this section, we review basic concepts of TCQ based limited feedback systems. Limited feedback schemes based on TCQ $[47,48,120]$ are recently gaining attention in massive multiple antenna FDD systems due to their reduced complexity compared to the conventional codebook approaches, in searching for an appropriate codeword. The quantization of CDI at the user is performed using TCQ, which consists of two key components: a trellis and a source constellation. At the user, a trellis path that gives the minimum squared Euclidean distance to the CDI is selected using the Viterbi algorithm. The input bit sequence corresponding to the selected path is then fed back to the BS using a low-rate feedback link. At the BS, a convolutional coder is implemented to decode the input bit sequence of the selected path to obtain the corresponding output sequence. The output sequence is then mapped to the source constellation to obtain the quantized CDI at the BS. Thus, the decoder and encoder of the TCM scheme are used, respectively, to quantize and reconstruct a channel vector in the TCQ method.

The block diagram of the feedback process is shown in Fig. 6.1, where the perfectly estimated channel, $\tilde{\mathbf{h}}$, is quantized by a user with the $N$-state trellis decoder and QPSK constellation. Note that, before implementing TCQ, the channel vector is normalized to obtain CDI, such that, $\tilde{\mathbf{u}}=\tilde{\mathbf{h}} /\|\tilde{\mathbf{h}}\|$. After implementing the TCQ, the input bit sequence, $\mathbf{b}$, (of length $n_{t}$ with QPSK constellation) of the selected path is fed back to the BS, where it is decoded to recover the corresponding output sequence (of length $2 n_{t}$ with QPSK constellation), using a convolutional coder. The output bit sequence is then mapped onto the QPSK constellation to reconstruct the quantized channel vector, $\tilde{\mathbf{q}}$. The complexity of the Viterbi algorithm is $O\left(L_{c} N n_{t}\right)$ [48], where $L_{c}$ is the total number of points in the source constellation and $N$ is the number of states in 


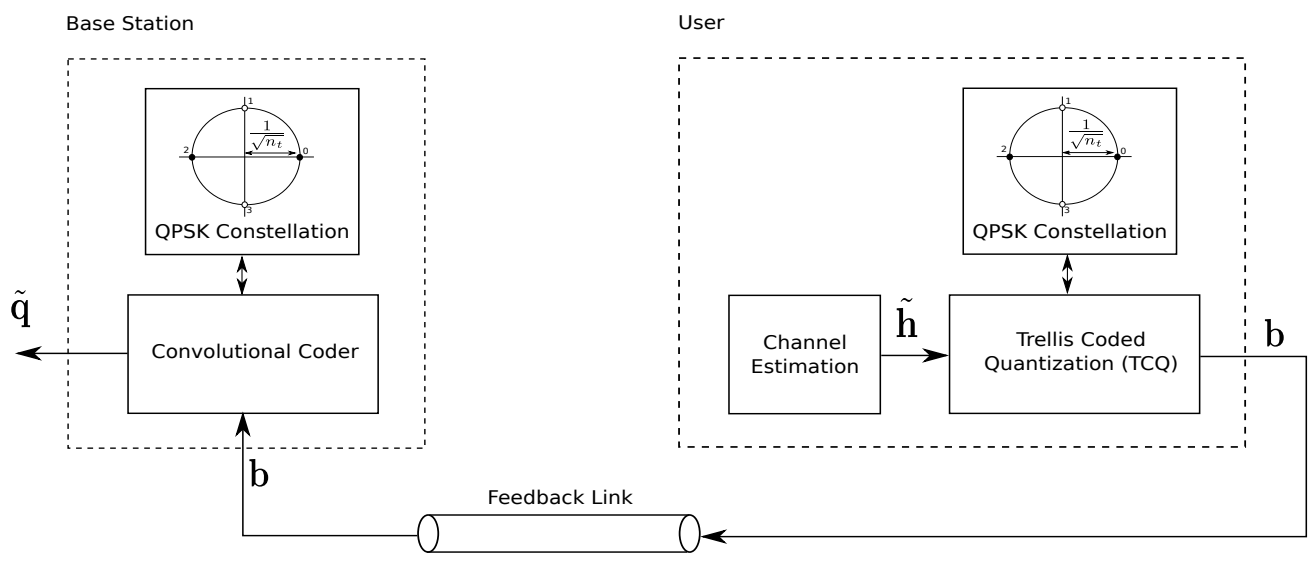

Figure 6.1: The block diagram of the TCQ feedback process with QPSK constellation.

the trellis.

Consider an example with a QPSK constellation along with a trellis structure having $N=4$ states. The QPSK constellation points are normalized by the number of transmit antennas, $n_{t}$. The normalized QPSK constellation and the 4-state, rate 1/2 Ungerboeck trellis structure are shown in Fig. 6.2 and Fig. 6.3, respectively. The decimal numbers $0,1,2$ and 3 (or 00, 01, 10, and 11 in binary) represent QPSK constellation points in Fig. 6.2. In Fig. 6.3, there are only two state-transitions from any given state. Each transition is mapped to a single QPSK point, hence each channel entry of $\tilde{\mathbf{u}}$ will be quantized with one of the two BPSK sub-constellations represented by black and white circles in Fig. 6.2. The main idea in TCQ encoding is to advance through an N-state trellis,

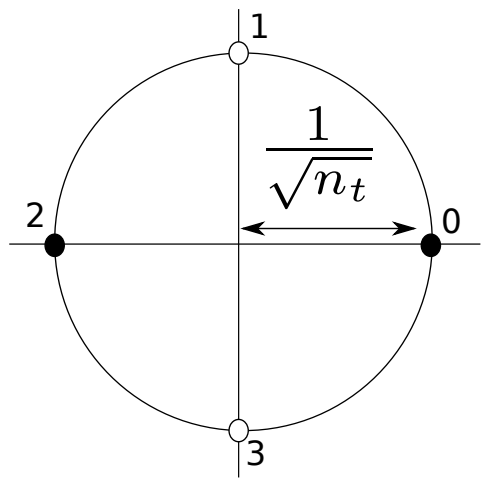

Figure 6.2: The normalized QPSK constellation points.

where the $n^{\text {th }}$ stage corresponds to the $n^{\text {th }}$ antenna channel. At any particular stage, there will be only $N$ survivor paths in the Viterbi algorithm. We label the paths by their respective output symbols. For example, starting from state 0 and moving through all the paths in the trellis to reach stage 3 , gives $2 N$ total paths. At stage 3, each state will have two paths terminating at it. As 
illustrated in Fig. 6.3, we have the following paths:

$$
\begin{aligned}
& {[0,0,0] \text { and }[2,1,2] \text { at state } 0,} \\
& {[0,0,2] \text { and }[2,1,0] \text { at state } 1,} \\
& {[0,2,1] \text { and }[2,3,3] \text { at state } 2,} \\
& {[0,2,3] \text { and }[2,3,1] \text { at state } 3 .}
\end{aligned}
$$

The path $\mathbf{p}_{2}=[2,1,2]$ terminating at state 0 corresponds to the output vector $\operatorname{out}\left(\mathbf{p}_{2}\right)=\left[\frac{-1}{\sqrt{n_{t}}}, \frac{+j}{\sqrt{n_{t}}}, \frac{-1}{\sqrt{n_{t}}}\right]$ from the QPSK constellation in Fig. 6.2 and the input bit sequence is $\mathbf{b}=[1,0,0]$. The user selects the best path from each state that gives minimum squared Euclidean distance to the normalized channel vector $\tilde{\mathbf{u}}$. The path metric can be defined at the $n^{\text {th }}$ stage as [48]

$$
\operatorname{metric}\left(\mathbf{p}^{(n)}\right)=\left\|\tilde{\mathbf{u}}^{(n)}-\operatorname{out}\left(\mathbf{p}^{(n)}\right)\right\|_{2}^{2},
$$

where $\tilde{\mathbf{u}}^{(n)}$ is a truncated normalized channel vector up to the $n^{\text {th }}$ channel entry. Equation (6.19) can be written recursively as

$$
\operatorname{metric}\left(\mathbf{p}^{(n)}\right)=\operatorname{metric}\left(\mathbf{p}^{(n-1)}\right)+\left|\tilde{u}^{(n)}-\operatorname{out}\left(p^{(n)}\right)\right|^{2},
$$

where $\tilde{u}^{(n)}$ and $p^{(n)}$ are the $n^{\text {th }}$ entries of $\tilde{\mathbf{u}}$ and $\mathbf{p}^{(n)}$. The solution to (6.20) that minimizes the path metric is obtained via a Viterbi algorithm. This enables us to determine the quantized CDI for large antenna numbers in a piecewise manner.

We assume that the TCQ scheme always starts from state 0, and thus it does not require the additional $\log _{2}(N)$ bits to be fed back to the BS to indicate the starting state. Due to large channel dimensions, we are dealing with a long

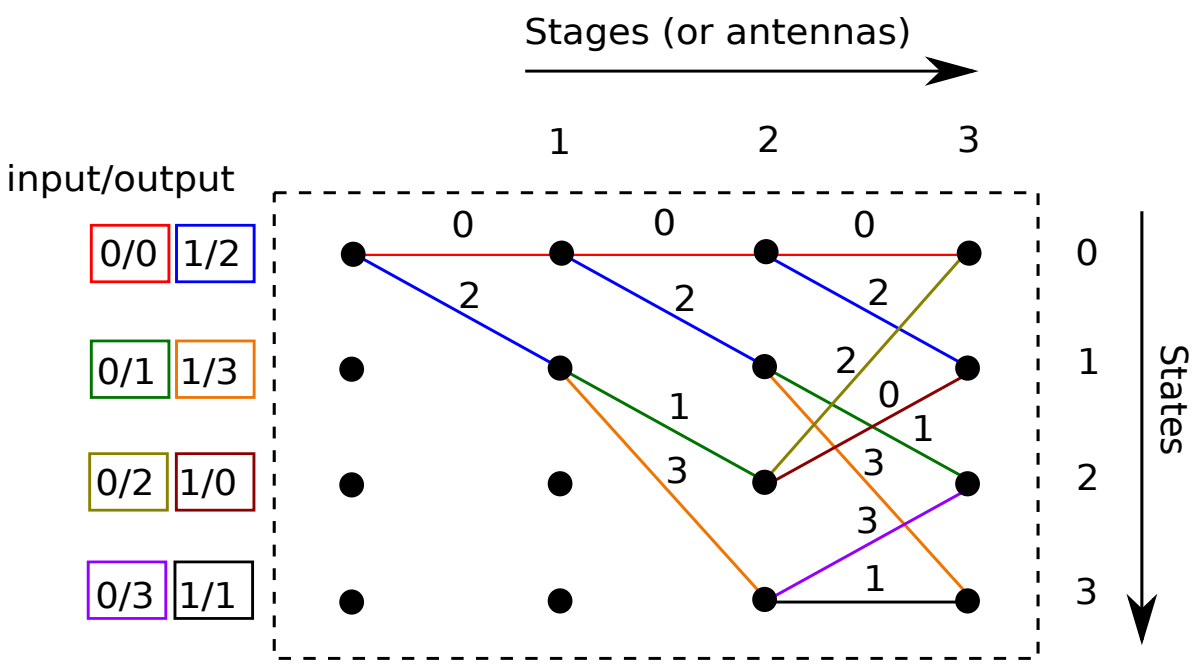

Figure 6.3: The 4-state, rate 1/2 Ungerboeck trellis structure. 


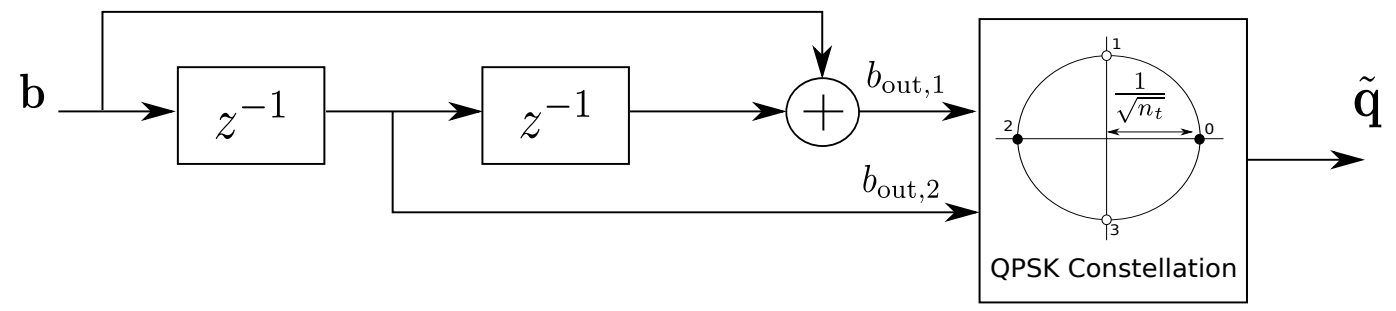

Figure 6.4: The convolutional coder corresponding to the 4 -state rate $1 / 2$ Ungerboeck trellis. In this figure, $\mathbf{b}$ is the input bit sequence, while $b_{\text {out }, 1}$ and $b_{\text {out }, 2}$ are the corresponding output bits for each entry of the input.

trellis structure therefore the quantization errors associated with always starting from state 0 is not significant.

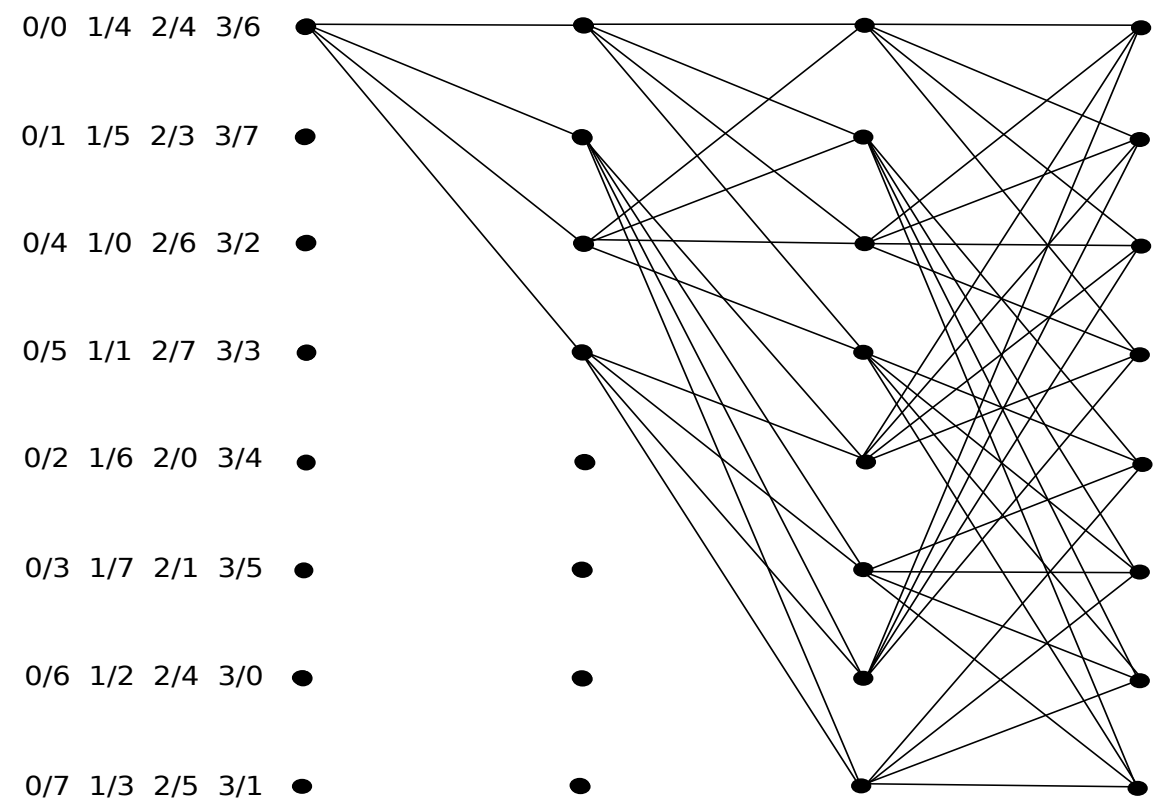

(a) 8 -state rate $2 / 3$ Ungerboeck trellis structure for $8 \mathrm{PSK}$ constellation.

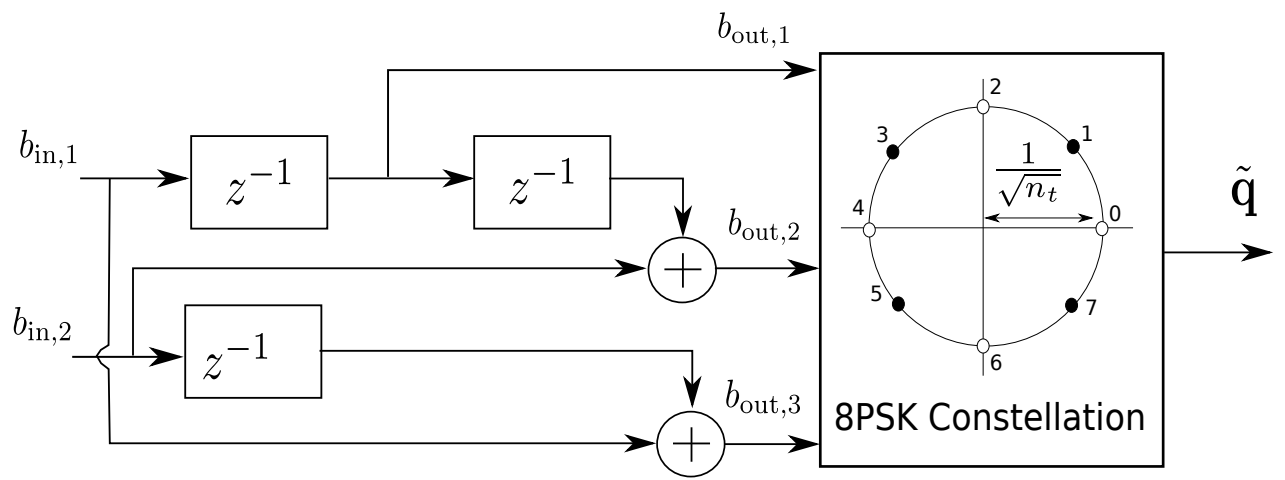

(b) Convolutional coder corresponding to 8 -state rate $2 / 3$ Ungerboeck trellis structure for 8PSK constellation.

Figure 6.5: Trellis structure and convolutional coder for limited feedback TCQ with 8PSK constellation. 
The source constellation plays an important role in deciding the required number of feedback bits. If the source constellation consists of $L_{c}$ number of symbols or constellation points, then the length of the input bit sequence (or the number of feedback bits) after TCQ becomes $n_{t}\left(\log _{2}\left(L_{c}\right)-1\right)$. Therefore, in order to keep the feedback overhead reasonable, we use QPSK and 8PSK source constellations, requiring $n_{t}$ and $2 n_{t}$ feedback bits, respectively. Another benefit is that all the constellation points in a PSK constellation are positioned with uniform angular spacing around a circle, yielding maximum phase separation between adjacent constellation points.

The performance of the TCQ based limited feedback scheme depends on the constellation size, the higher the constellation size the fewer the quantization errors. However, using the higher order source constellation increases the feedback overhead. The 8PSK source constellation consists of $L_{c}=8$ constellation points. The 8 -state, rate $2 / 3$ Ungerboeck trellis structure and corresponding convolutional coder are shown in Fig. 6.5(a) and Fig. 6.5(b), respectively.

The limited feedback TCQ method with 8PSK constellation is similar to QPSK constellation, where with the 8PSK constellation each channel entry is quantized by 2 bits, hence doubling the feedback overhead compared to the QPSK constellation.

\subsection{Differential TCQ Scheme for Temporally Cor- related Channels}

Here, we discuss a differential extension to the TCQ method discussed in Section 6.5 which allows us to quantize the temporally correlated massive MISO CDI after successively transforming (translating and scaling) the source constellation following each feedback interval. This repositioning of the source constellation allows the feedback process to track the channel of each antenna over time. We assume a single Viterbi block at each user ${ }^{1}$.

\subsubsection{Transformed Source Constellation}

The basic idea in the differential TCQ is to keep track of the selected source constellation points at each trellis stage and define a new constellation for the next feedback centered around the previously selected constellation points. For the first feedback interval, we use the TCQ method described in Section 6.5. Starting with the second feedback interval, the constellation points are transformed for all the stages at time $t$ such that the previously selected constellation points becomes the new centers of the transformed constellations at time $t+1$.

\footnotetext{
${ }^{1}$ Unlike [48], multiple blocks of parallel coherent decoders with different amplitude scalings and phase rotations are not used, as this offers limited gain.
} 
For example, all four points in the original non-normalized QPSK constellation $[1, j,-1,-j]$ are transformed into new points using translation and scaling methods, to be discussed. Apart from this modification, the quantization process follows the TCQ approach discussed in Section 6.5.

An example of the proposed method with QPSK constellation for the first 3 stages with $N=4$ is shown in Fig. 6.6, where the first feedback at $t=0$ is illustrated at the top with red dots representing the selected QPSK points at each stage for the selected path $[2,1,2]$. At $t=1$, the transformed QPSK constellation at each stage is shown in the middle of Fig. 6.6 for the selected path $[0,2,1]$. At any given stage, the transformed QPSK constellation is centered around the previously selected QPSK point $(t=0)$, with a scaling factor $\kappa_{c}$ (derived in Section 6.6.2). It should be stressed that this proposed transformation of the QPSK constellation is achieved at both BS and user without sharing any additional information through the feedback link. The BS also transforms the QPSK constellation after each feedback such that the new constellation at each stage is centered around the previously selected QPSK point.

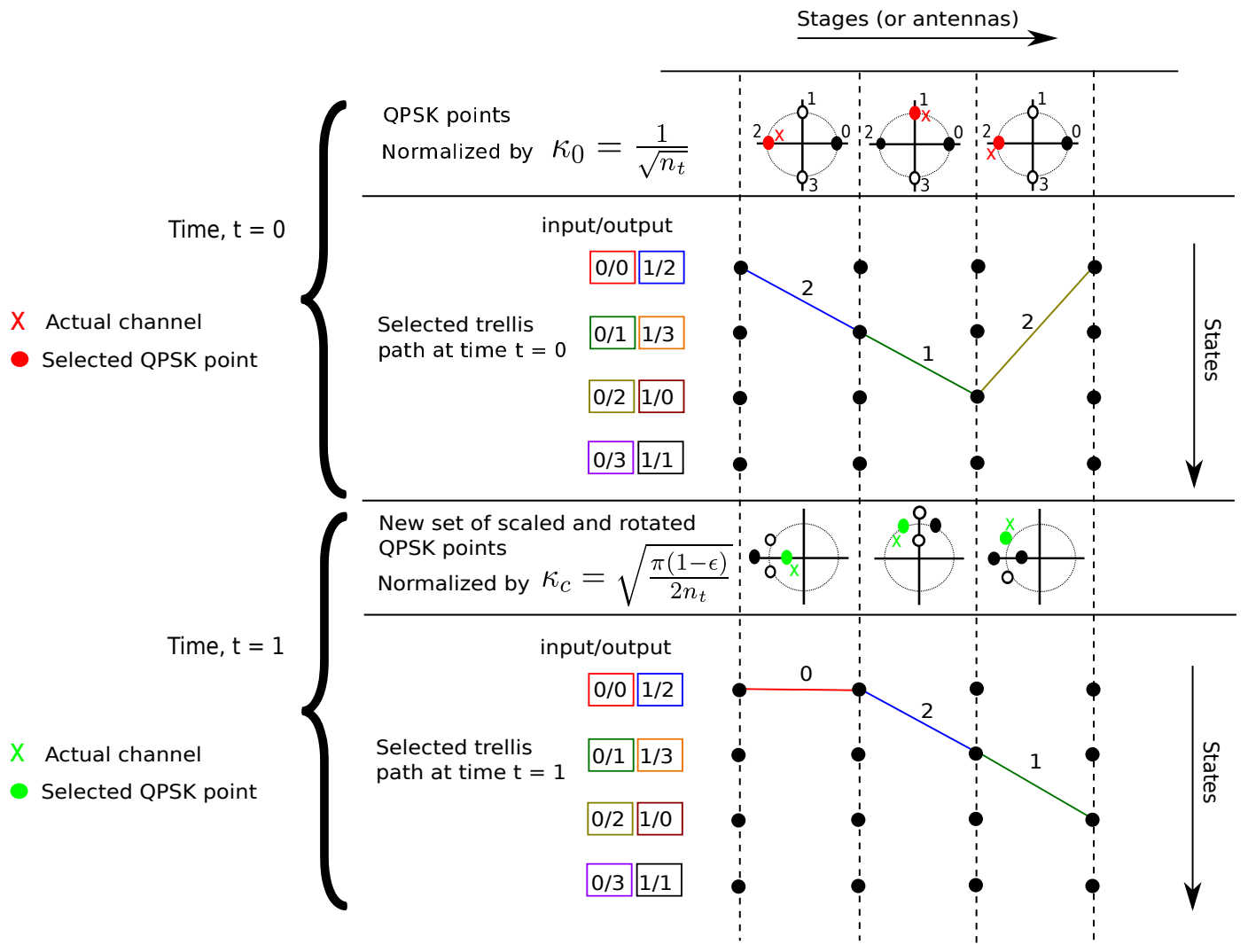

Figure 6.6: An example of scaled and rotated QPSK constellation points at $t=1$ centered around the previously selected point at $t=0$ for up to three antenna channels. 


\subsubsection{D Translation and Scaling Techniques}

The TCQ method for massive MISO channels uses the Viterbi algorithm to quantize the channel and unlike conventional MISO systems, it does not maintain a codebook that is scaled and rotated to the desired location. Hence, we introduce 2D translation and scaling transformations for the non-normalized source constellation (i.e., $[1, j,-1,-j]$, for QPSK) at each stage in the trellis.

Applying the transformation (6.21) to each point in the source constellation, we ensure that the previously selected constellation point, $\hat{x}[t-1]=\hat{a}[t-1]+$ $j \hat{b}[t-1]$ is at the center of the transformed constellation. The translation of the $i^{\text {th }}$ constellation point, $x_{i}=a+j b$, along with scaling by $\kappa_{c}$ is given by [121]

$$
\left[\begin{array}{c}
\tilde{a}[t] \\
\tilde{b}[t] \\
1
\end{array}\right]=\left[\begin{array}{ccc}
1 & 0 & \hat{a}[t-1] \\
0 & 1 & \hat{b}[t-1] \\
0 & 0 & 1
\end{array}\right]\left[\begin{array}{ccc}
\kappa_{c} & 0 & 0 \\
0 & \kappa_{c} & 0 \\
0 & 0 & 1
\end{array}\right]\left[\begin{array}{l}
a \\
b \\
1
\end{array}\right],
$$

where $\tilde{x}_{i}[t]=\tilde{a}[t]+j \tilde{b}[t]$ is the $i^{\text {th }}$ transformed constellation point. All points in the constellation are translated and scaled using (6.21). Note that scaling and translation are performed on the non-normalized source constellation points.

In order to track the $n^{\text {th }}$ channel entry, $\tilde{u}^{(n)}$, over time, the scaling factor, $\kappa_{c}$, needs to be carefully designed, such that $\tilde{u}^{(n)}$ lies close to the transformed constellation points. We can define the mean channel variation due to the temporal correlation for the $n^{\text {th }}$ antenna as the mean Euclidean distance between the current and the previous normalized channel values, that is

$$
\begin{aligned}
\mathrm{d}_{\text {Euclidean }} & =\mathbb{E}\left[\left|\tilde{u}^{(n)}[t-1]-\tilde{u}^{(n)}[t]\right|\right], \\
& =\mathbb{E}\left[\left|\frac{\tilde{h}^{(n)}[t-1]}{\|\tilde{\mathbf{h}}[t-1]\|}-\frac{\tilde{h}^{(n)}[t]}{\|\tilde{\mathbf{h}}[t]\|}\right|\right],
\end{aligned}
$$

where $\tilde{h}^{(n)}[t-1]$ and $\tilde{h}^{(n)}[t]$ denote the $n^{\text {th }}$ entry of the channel vectors $\tilde{\mathbf{h}}[t-1]$ and $\tilde{\mathbf{h}}[t]$, respectively. Due to channel hardening [36] caused by the large dimensions of $\tilde{\mathbf{h}}[t]$ and $\tilde{\mathbf{h}}[t-1], \frac{\|\tilde{\mathbf{h}}[t]\|^{2}}{n_{t}}$ and $\frac{\|\tilde{\mathbf{h}}[t-1]\|^{2}}{n_{t}}$ approach 1 , and thus

$$
\frac{\tilde{h}^{(n)}[t]}{\|\tilde{\mathbf{h}}[t]\|}=\frac{\tilde{h}^{(n)}[t] / \sqrt{n_{t}}}{\sqrt{\|\tilde{\mathbf{h}}[t]\|^{2} / n_{t}}} \approx \frac{\tilde{h}^{(n)}[t]}{\sqrt{n_{t}}} .
$$


This allows us to approximate (6.23) as

$$
\begin{aligned}
\mathrm{d}_{\text {Euclidean }} & \approx \frac{1}{\sqrt{n_{t}}} \mathbb{E}\left[\left|\tilde{h}^{(n)}[t-1]-\tilde{h}^{(n)}[t]\right|\right] \\
& =\frac{1}{\sqrt{n_{t}}} \mathbb{E}\left[\left|\tilde{h}^{(n)}[t-1]-\left(\epsilon \tilde{h}^{(n)}[t-1]+\sqrt{1-\epsilon^{2}} y^{(n)}[t]\right)\right|\right], \\
& =\frac{1}{\sqrt{n_{t}}} \mathbb{E}\left[\left|\tilde{h}^{(n)}[t-1](1-\epsilon)-\sqrt{1-\epsilon^{2}} y^{(n)}[t]\right|\right],
\end{aligned}
$$

where $y^{(n)}[t]$ denotes the $n^{\text {th }}$ entry of the vector $\mathbf{y}[t]$ in (2.13). Denoting $\Re\left\{\tilde{h}^{(n)}[t-\right.$ $1]\}$ and $\Im\left\{\tilde{h}^{(n)}[t-1]\right\}, \Re\left\{y^{(n)}[t]\right\}$ and $\Im\left\{y^{(n)}[t]\right\}$ as the real and imaginary parts of $\tilde{h}^{(n)}[t-1]$ and $y^{(n)}[t]$, respectively, we have

$$
\mathrm{d}_{\text {Euclidean }} \approx \frac{1}{\sqrt{n_{t}}} \mathbb{E}\left[\sqrt{X^{2}+Y^{2}}\right]
$$

where

$$
X=(1-\epsilon) \Re\left\{\tilde{h}^{(n)}[t-1]\right\}-\sqrt{1-\epsilon^{2}} \Re\left\{y^{(n)}[t]\right\},
$$

and

$$
Y=(1-\epsilon) \Im\left\{\tilde{h}^{(n)}[t-1]\right\}-\sqrt{1-\epsilon^{2}} \Im\left\{y^{(n)}[t]\right\} .
$$

Since $\Re\left\{\tilde{h}^{(n)}[t-1]\right\}, \Re\left\{y^{(n)}[t]\right\}, \Im\left\{\tilde{h}^{(n)}[t-1]\right\}$ and $\Im\left\{y^{(n)}[t]\right\}$ are independent and distributed according to the normal distribution $\mathcal{N}(0,1 / 2)$, the random variables $X$ and $Y$ are $\mathcal{N}(0,1-\epsilon)$. Therefore, $Z=\sqrt{X^{2}+Y^{2}}$ is Rayleigh distributed with scale parameter $\sigma=\sqrt{1-\epsilon}$, and the mean value $\sqrt{1-\epsilon} \sqrt{\pi / 2}$ [122]. We can now write $(6.28)$ as

$$
\mathrm{d}_{\text {Euclidean }} \approx \sqrt{\frac{\pi(1-\epsilon)}{2 n_{t}}}
$$

The mean channel variation includes the effects of both temporal correlation and the number of transmit antennas at the BS. Therefore, in order to track slowly varying channels and to have the source constellation points closer to each other for fine quantization, we use (6.30) as the scaling value, $\kappa_{c}$, after the first feedback interval, such that

$$
\kappa_{c}=\sqrt{\frac{\pi(1-\epsilon)}{2 n_{t}}}, \quad t>0 .
$$

The initial scaling factor for the source constellation, $\kappa_{0}=1 / \sqrt{n_{t}}$, is used only for the first feedback interval. Following the first feedback interval, the value used to scale the source constellation for the remaining feedback intervals is $\kappa_{c}$. In (6.31), the larger the value of $n_{t}$, the smaller the value of $\kappa_{c}$, i.e., there is less variation in the channel. Both $\mathrm{BS}$ and the user compute the scaling value 
in (6.31) using the temporal correlation coefficient (assumed to be known at the BS and user) and the number of BS antennas. This value needs not to be computed after each feedback interval as long as the temporal correlation statistic of the channel remains the same.

The differential TCQ method discussed in Section 6.6 for the temporally correlated MISO channel does not increase the feedback overhead and performance improvements are achieved via the systematic translation and scaling of the QPSK constellation. It is important to note that the NTCQ differential scheme [48] depends on two crucial operations: a) finding the null space of the temporally correlated channel vector before quantization and b) selecting the appropriate weights after quantization. In contrast, the differential TCQ scheme relies solely on the transformation of the source constellation and does not require finding optimal weights, thus reducing the complexity. The differential TCQ scheme also reduces the feedback overhead as it does not have to feedback additional bits (for optimal weights) to the BS. We compare the performance of the two schemes in Section 6.8.

\subsection{TCQ Scheme for Spatially Correlated Chan- nels}

In this section, we present a TCQ scheme to quantize spatially correlated massive MISO channels. In conventional MIMO systems, the codebook design for a spatially correlated channel is fixed where codebook entries are directed towards dominant eigenvectors of the transmit correlation matrix [45]. As discussed in Section 6.4, massive MISO systems using codebook based limited feedback techniques are not practically feasible. Therefore, an alternate method is needed to quantize a spatially correlated channel. For this purpose, an adaptive TCQ based limited feedback method is developed in [48] that decorrelates the channel at the user before quantization. The drawback of this method is that it requires the knowledge of the spatial correlation matrix at the transmitter. In this study, the spatially correlated massive MISO channel is modeled by

$$
\hat{\mathbf{h}}=\mathbf{h} \mathbf{R}_{\mathrm{t}}^{1 / 2}
$$

where $\mathbf{h}$ is an $1 \times n_{t}$ vector with entries distributed according to $\mathcal{C N}(0,1) . \mathbf{R}_{t}$ is the spatial correlation matrix of the transmitter. The entries of $\mathbf{R}_{\mathrm{t}}, r_{\mathrm{t}}^{i j}$, follow an exponential correlation model given by $[55,116]$

$$
r_{\mathrm{t}}^{i j}=z_{\mathrm{t}}^{d^{i j}}
$$


where $d^{i j}$ is the distance between the antenna $i$ and $j . z_{\mathrm{t}}$ is a spatial correlation coefficient with $0 \leq z_{\mathrm{t}} \leq 1$, where 0 represents no spatial correlation and 1 represents a fully correlated channel. We consider ULA and URA antenna topologies at the BS. The one-dimensional ULA is much larger than the compact URA, therefore the latter is practically more suitable for Massive MISO. We constrain the dimensions of the URA, such that for a given value of $n_{t}$, the dimensions of the URA are $\sqrt{n_{t}} \times \sqrt{n_{t}}$. In order to design a TCQ scheme for
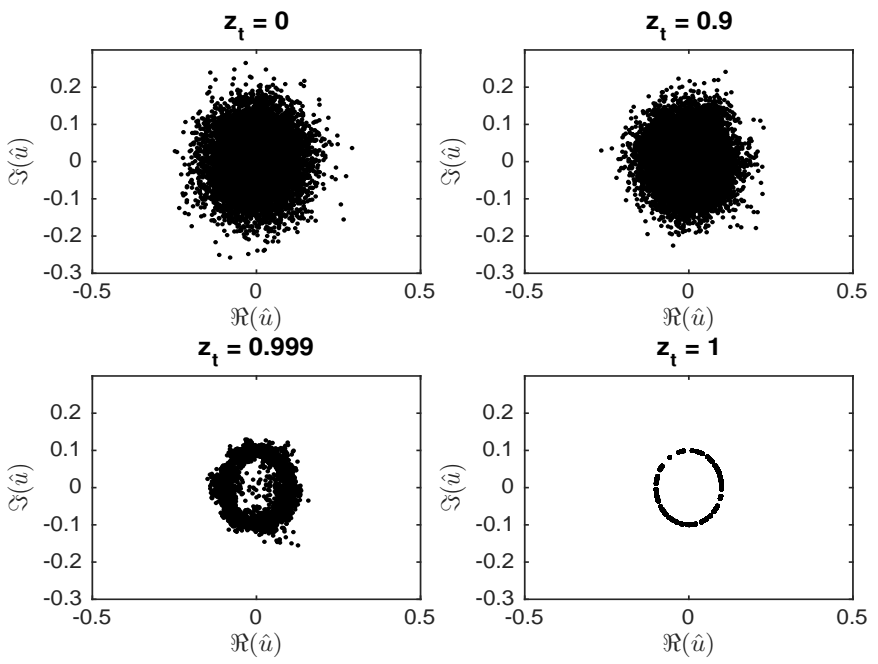

Figure 6.7: Distribution of channel entries of the normalized channel for $n_{t}=$ 100 and for different values of $z_{t}$.

spatially correlated massive MISO channels, we first analyze the impact of $z_{\mathrm{t}}$ on the distribution of channel entries. For this purpose, we plot the channel entries of the normalized spatially correlated channel with $n_{t}=100$, over a large number of channel realizations in Fig. 6.7, for various values of $z_{\mathrm{t}}$. If $\hat{u}^{(i)}$ is the $i^{\text {th }}$ element of $\hat{\mathbf{h}} /\|\hat{\mathbf{h}}\|$ and $\hat{h}^{(i)}$ is the $i^{\text {th }}$ element of $\hat{\mathbf{h}}$, then in the i.i.d. case $\left(z_{\mathrm{t}}=0\right),\left|\hat{u}^{(i)}\right|^{2}=\left|\hat{h}^{(i)}\right|^{2} / \sum_{j=1}^{n_{t}}\left|\hat{h}^{(j)}\right|^{2}$ and since each $\left|\hat{h}^{(j)}\right|^{2}$ is a standard exponential, $\left|\hat{u}^{(i)}\right|^{2}$ has a Beta distribution. Hence, the amplitude of $\hat{u}^{(i)}$ is the square root of the a Beta variable with range, $0<\left|\hat{u}^{(i)}\right|<1$. Since the phase of $\hat{h}^{(i)}$ is uniform on $[0,2 \pi]$, it follows that the normalized channel entries are isotropic, as shown by the circular pattern in Fig. 6.7. At the opposite extreme, when $z_{t}=1$, we have perfectly or fully correlated entries. Denoting the $i^{\text {th }}$ element of the fully correlated channel by $\hat{u}_{f}^{(i)}$, where $\hat{u}_{f}^{(i)}=\hat{u}_{f} \forall i$, then $\left|\hat{u}_{f}^{(i)}\right|^{2}=\left|\hat{h}_{f}^{(i)}\right|^{2} / \sum_{j=1}^{n_{t}}\left|\hat{h}_{f}^{(j)}\right|^{2}=1 / n_{t}$. Hence, for $z_{\mathrm{t}}=1$, the channel entries lie on a circle of radius $1 / \sqrt{n_{t}}$, as shown in Fig. 6.7. Clearly as $z_{t}$ varies from 0 to 1 , the channel entry distribution changes from a circular spread with random radius to a fixed circle of radius $1 / \sqrt{n_{t}}$.

From Fig. 6.7, we also make the following observation. As, the value of $z_{t}$ increases, the distribution of entries of the normalized spatially correlated chan- 
nel shows less spread compared to i.i.d. Rayleigh channels $\left(z_{\mathrm{t}}=0\right)$. However, this trend is mainly visible for high values of $z_{t}$, such that $z_{\mathrm{t}} \rightarrow 1$. Even with $z_{\mathrm{t}}=0.9$, the spread of the normalized channel entries is similar to the $z_{t}=0$ case.

Motivated by these observations, we develop a TCQ scheme for highly spatially correlated MISO channels by considering the fully correlated channel. We set the scaling parameter, $\hat{\kappa}_{c}$, for the source constellation as MMSE between the entries of the fully correlated normalized channel $\left(z_{\mathrm{t}}=1\right)$ and the scaled source constellation, given by

$$
\hat{\kappa}_{c}=\arg \min _{\substack{0 \leq i \leq L_{c} \\ \hat{\kappa}_{c}>0}} \mathbb{E}\left[\left|\hat{u}_{f}-\hat{\kappa}_{c} x_{i}\right|^{2}\right]
$$

where $x_{i}$ corresponds to the $i^{\text {th }}$ point in the source constellation. The scaling parameter value is selected from the set of positive real numbers, such that, $\hat{\kappa}_{c}>$ 0 . The solution to (6.34) is equal to the standard deviation of the channel entries $\hat{u}_{f}$ given by $1 / \sqrt{n_{t}}$. Therefore, for the TCQ method for spatially correlated channels, we set the scaling parameter for the source constellation as

$$
\hat{\kappa}_{c}=\frac{1}{\sqrt{n_{t}}}
$$

The TCQ method for spatially correlated channels uses the TCQ method discussed in Section 6.5 to quantize the channel, $\hat{\mathbf{h}}$ in (6.32), where the source constellation (either QPSK or 8PSK) used is scaled by $\hat{\kappa}_{c}$ and is fixed across all the stages in the trellis. Although, we have assumed the fully coorelated channel for computing $\hat{\kappa}_{c}$, this scaling value has shown to work well in the numerical section (Section 6.8) for highly correlated channels (where $z_{\mathrm{t}}<1$ ).

The block diagram of the TCQ technique for spatially correlated channels, at the user/receiver is shown in Fig. 6.8. Note that as discussed in Section 6.5 and 6.6 , the channel vector $\hat{\mathbf{h}}$ is normalized before TCQ encoding. The channel reconstruction at the transmitter is same as explained in Section 6.5.

Unlike [48], the TCQ method for spatially correlated channels does not decorrelate the channel at the user in order to quantize $\mathbf{h}$, instead it directly quantizes the channel $\hat{\mathbf{h}}$. Therefore, unlike [48], it does not require the knowledge of the correlation matrix at the transmitter to reconstruct the correlated channel, hence reducing the feedback overhead of additional information to the transmitter. The performance of the TCQ scheme for spatially correlated channels is demonstrated in Section 6.8 for ULA and URA topologies. 


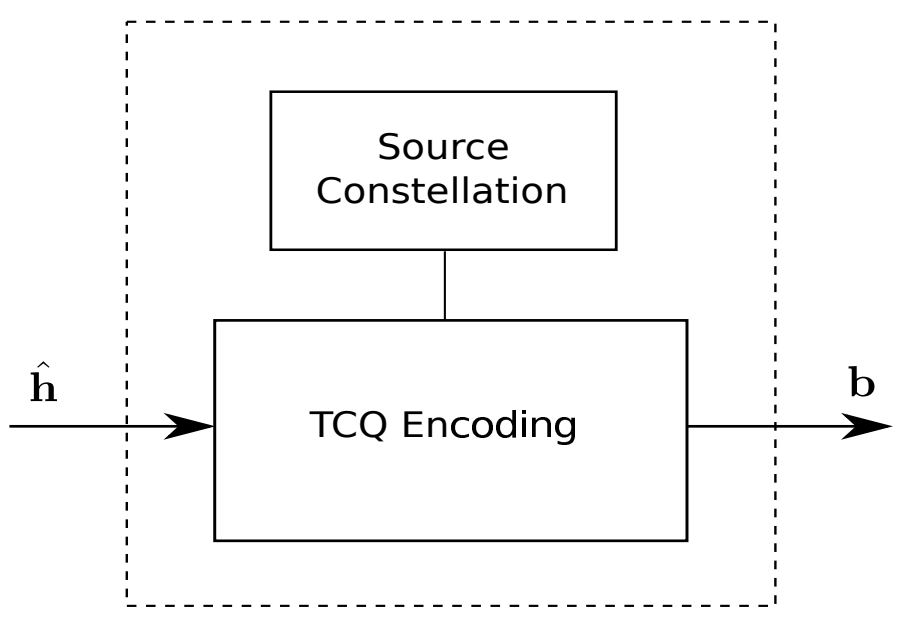

Figure 6.8: The block diagram of channel quantization at the user for spatially correlated channels.

\subsection{Numerical Results and Discussion}

In this section, we present simulation results for TCQ schemes for temporally and spatially correlated massive MISO channels, discussed in Section 6.6 and Section 6.7, respectively. We compare the performance of these schemes with the differential NTCQ and the adaptive NTCQ techniques in [48]. We consider three types of channels: the temporally correlated channel (2.13), the spatially correlated channel (2.11) and the standardized WINNER II channel (2.16).

In the case of a MU MISO system we assume a constant ratio $q=n_{t} / K=10$. As discussed in the previous chapter, the feedback interval is set to $5 \mathrm{~ms}$. The carrier frequency is $2.5 \mathrm{GHz}$. We assume that the feedback link is lossless with zero delay in the simulations. For a fair comparison between the TCQ schemes and the NTCQ schemes [48], the source constellations used are QPSK and 8PSK with $N=4$ and $N=8$, respectively. A single block of the trellis decoder is considered at the receiver.

For the QPSK source constellation, we use the 4 state rate $1 / 2$ Ungerboeck trellis structure shown in Fig. 6.3 and the corresponding convolutional coder presented in Fig. 6.4. For the 8PSK source constellation, we employ the 8 state rate $2 / 3$ Ungerboeck trellis structure shown in Fig. 6.5(a) and the corresponding convolutional coder presented in Fig. 6.5(b).

In the figures, we refer to the differential NTCQ method of [48] for temporally correlated channels as "Diff. NTCQ". The differential TCQ method is referred to as "Diff. TCQ". Similarly, for spatially correlated channels, we refer to the TCQ scheme in Section 6.7 as "Spatial corr." and adaptive NTCQ scheme in [48] is referred as "Adaptive spatial corr.". 


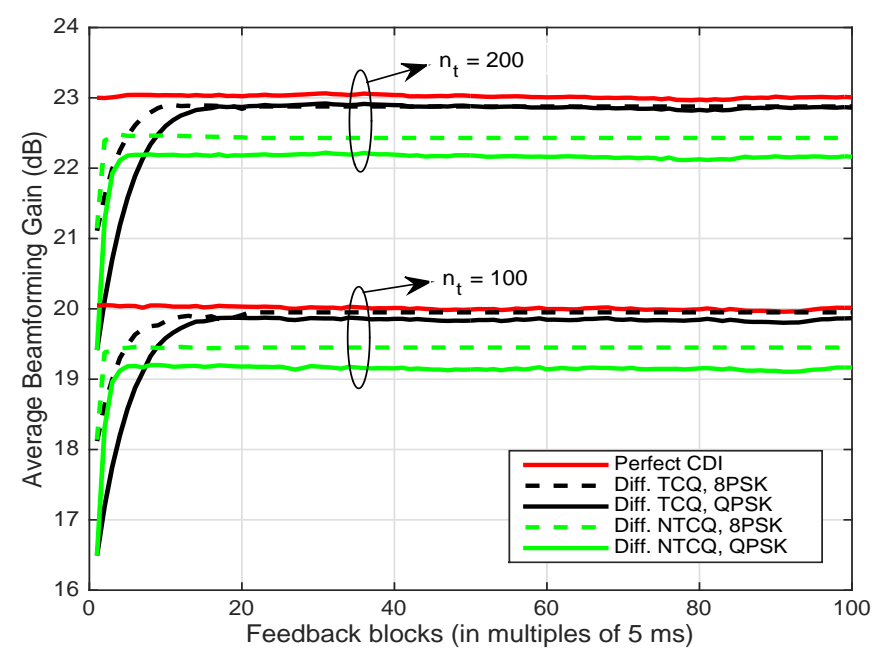

Figure 6.9: Average beamforming gain versus feedback intervals with $n_{t}=100$ and $n_{t}=200$ for $\epsilon=0.9881(v=3 \mathrm{~km} / \mathrm{h})$.

\subsubsection{Temporally Correlated Channels}

For temporally correlated channels, we use the channel model in (2.13) and present the performance of the proposed differential TCQ scheme in MU massive MISO systems.

\section{Beamforming gain}

We use the average beamforming gain metric [48] defined as $\mathbb{E}\left[|\tilde{\mathbf{h}}[t] \tilde{\mathbf{q}}[t]|^{2}\right]$, to evaluate the performance of a MISO system. The average beamforming gain shows how well quantized channels match actual channels. Fig. 6.9 shows average beamforming gain results in $\mathrm{dB}$ against 100 feedback blocks (size of each block is $5 \mathrm{~ms})$ for the user velocity $v=3 \mathrm{~km} / \mathrm{h}(\epsilon=0.9881)$ with $n_{t}=100$ and $n_{t}=200$. The differential TCQ scheme provides approximately $1 \mathrm{~dB}$ gain compared to [48] for both $n_{t}=100$ and $n_{t}=200$ with QPSK constellation. The average beamforming gain with 8PSK constellation is higher than the QPSK constellation because the former uses 2 bits to quantize each antenna channel, resulting in smaller quantization errors.

It is important to note that the differential TCQ scheme requires $n_{t}$ and $2 n_{t}$ feedback bits with QPSK and 8PSK constellations, respectively, whereas an additional 4 bits are required in the differential NTCQ method [48] for obtaining the optimal weights corresponding to quantized CDI, hence increasing the total number of feedback bits to $n_{t}+4$ and $2 n_{t}+4$ for QPSK and 8PSK, respectively. Thus, in addition to the improved beamforming gain, the differential TCQ method also reduces the feedback overhead. 


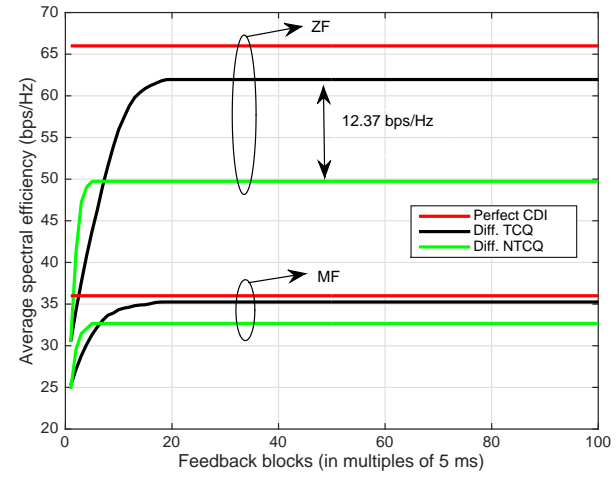

(a) QPSK

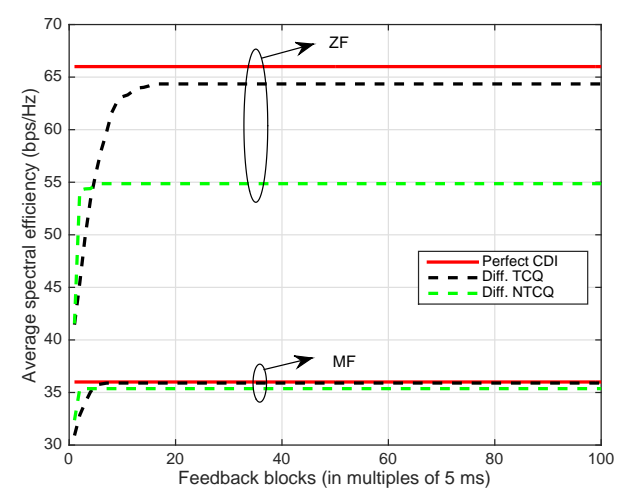

(b) 8 PSK

Figure 6.10: Average spectral efficiency versus time with $n_{t}=100$ and $\epsilon=$ $0.9881(v=3 \mathrm{~km} / \mathrm{h})$.

\section{MU spectral efficiency and SINR performance}

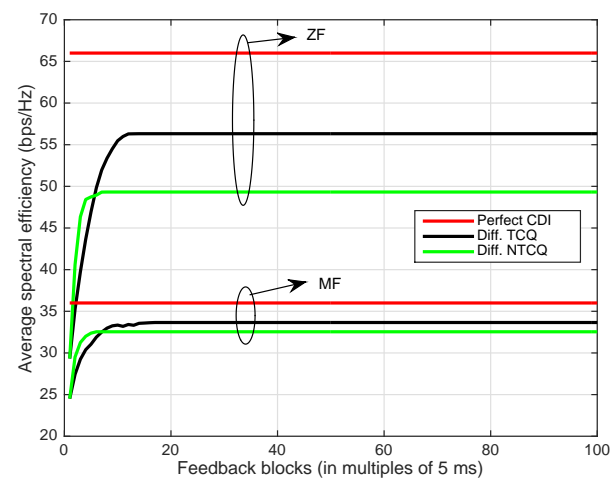

(a) QPSK

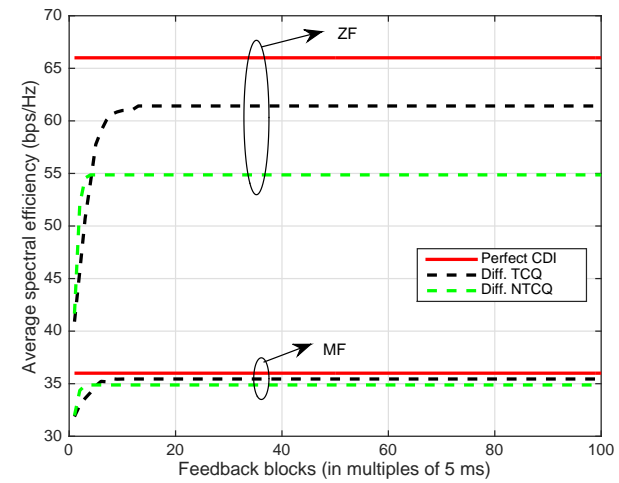

(b) 8 PSK

Figure 6.11: Average spectral efficiency versus time with $n_{t}=100$ and $\epsilon=$ $0.9672(v=5 \mathrm{~km} / \mathrm{h})$.

For MU MISO systems, we use ZF precoding and MF precoding to compute average SINR (3.7) and spectral efficiency (3.6) results. Figs. 6.10(a) and 6.10(b) show the average spectral efficiency versus feedback interval for a MU massive MISO system at SNR, $\rho=10 \mathrm{~dB}$, with $n_{t}=100$ and $v=3 \mathrm{~km} / \mathrm{h}$ $(\epsilon=0.9881)$ with QPSK and 8PSK constellations, respectively. ZF precoding outperforms MF precoding in the temporally correlated channels. We also note that the spectral efficiency of the differential TCQ scheme exceeds that of the differential NTCQ method with both QPSK and 8PSK constellations. In Fig. 6.10(a), the differential TCQ scheme provides nearly $13 \mathrm{bps} / \mathrm{Hz}$ and $2 \mathrm{bps} / \mathrm{Hz}$ average spectral efficiency gains compared to the differential NTCQ method with QPSK constellation, for ZF and MF precoding schemes, respectively. A similar trend is seen in Fig. 6.10(b) with 8PSK constellation for ZF 


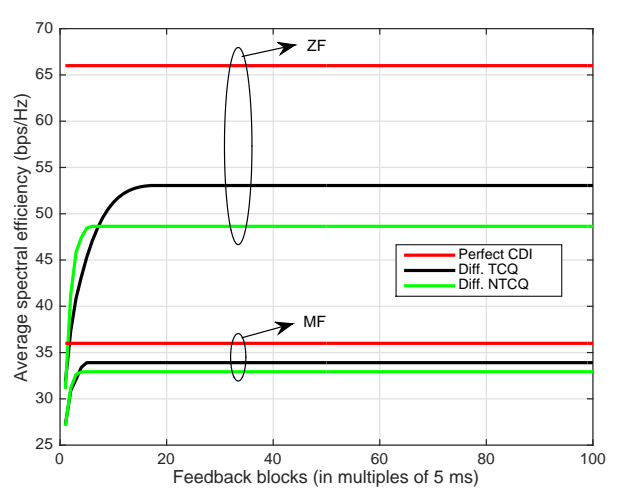

(a) QPSK

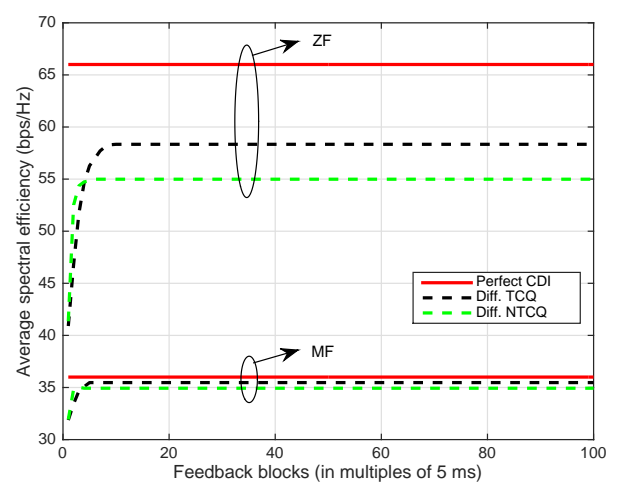

(b) 8 PSK

Figure 6.12: Average spectral efficiency versus time with $n_{t}=100$ and $\epsilon=$ $0.9363(v=7 \mathrm{~km} / \mathrm{h})$.

precoding, but for MF precoding the performance gap between the differential TCQ scheme and the differential NTCQ scheme decreases. As the velocity increases to $v=5 \mathrm{~km} / \mathrm{h}$, the variations in the channel also increase which leads to a higher spectral efficiency loss as seen in Fig. 6.11(a) and Fig. 6.11(b). A similar trend is seen in Fig. 6.12(a) and Fig. 6.12(b), for $v=7 \mathrm{~km} / \mathrm{h}$. It is noticed that as the velocity of the users increases, the differential TCQ scheme exhibits higher spectral efficiency loss compared to differential NTCQ scheme with ZF precoding, but still performs better than the differential NTCQ scheme.

Table 6.1 shows the average spectral efficiency of the differential TCQ scheme and the differential NTCQ scheme for various speed values with ZF precoding and QPSK constellation. The differential TCQ yields better average spectral efficiency for slow speeds compared to the differential NTCQ scheme. However, at $v=13 \mathrm{~km} / \mathrm{h}$, both schemes have the same performance.

Figure 6.13 shows the average spectral efficiency against different values of $q$ for the differential TCQ and differential NTCQ schemes using a QPSK constellation with $n_{t}=100, v=3 \mathrm{~km} / \mathrm{h}$ and $\rho=10 \mathrm{~dB}$. The proposed scheme achieves better spectral efficiency than the differential NTCQ scheme, as the former yields less quantization error. It is seen that as more users are added to the system, the performance gain with the proposed scheme increases compared to the differential NTCQ scheme.

The per user SINR CDF is shown in Fig. 6.14 at SNR, $\rho=10 \mathrm{~dB}$ with QPSK constellation. For ZF precoding, the SINR CDF of the differential TCQ scheme has a long-tail. This is because the basic TCQ method (discussed in Section 6.5) is used for the first feedback yielding low SINR performance, but with time the SINR performance improves using the differential TCQ method. The SINR CDFs confirm the spectral efficiency results i.e., the mean SINR of the differential TCQ scheme is greater than the differential NTCQ method for 
Table 6.1: Average spectral efficiency of the proposed differential TCQ scheme and the differential NTCQ scheme in [48] with ZF precoding and QPSK constellation for $n_{t}=100, q=10$ and $\rho=10 \mathrm{~dB}$.

\begin{tabular}{|c|c|c|c|c|c|}
\hline & $v=3 \mathrm{~km} / \mathrm{h}$ & $v=5 \mathrm{~km} / \mathrm{h}$ & $v=7 \mathrm{~km} / \mathrm{h}$ & $v=10 \mathrm{~km} / \mathrm{h}$ & $v=13 \mathrm{~km} / \mathrm{h}$ \\
& $\epsilon=0.9881$ & $\epsilon=0.9672$ & $\epsilon=0.9363$ & $\epsilon=0.8721$ & $\epsilon=0.7895$ \\
\hline Diff. TCQ & $60 \mathrm{bps} / \mathrm{Hz}$ & $55 \mathrm{bps} / \mathrm{Hz}$ & $52 \mathrm{bps} / \mathrm{Hz}$ & $45 \mathrm{bps} / \mathrm{Hz}$ & $39 \mathrm{bps} / \mathrm{Hz}$ \\
\hline Diff. NTCQ & $50 \mathrm{bps} / \mathrm{Hz}$ & $48 \mathrm{bps} / \mathrm{Hz}$ & $47 \mathrm{bps} / \mathrm{Hz}$ & $44 \mathrm{bps} / \mathrm{Hz}$ & $39 \mathrm{bps} / \mathrm{Hz}$ \\
\hline
\end{tabular}

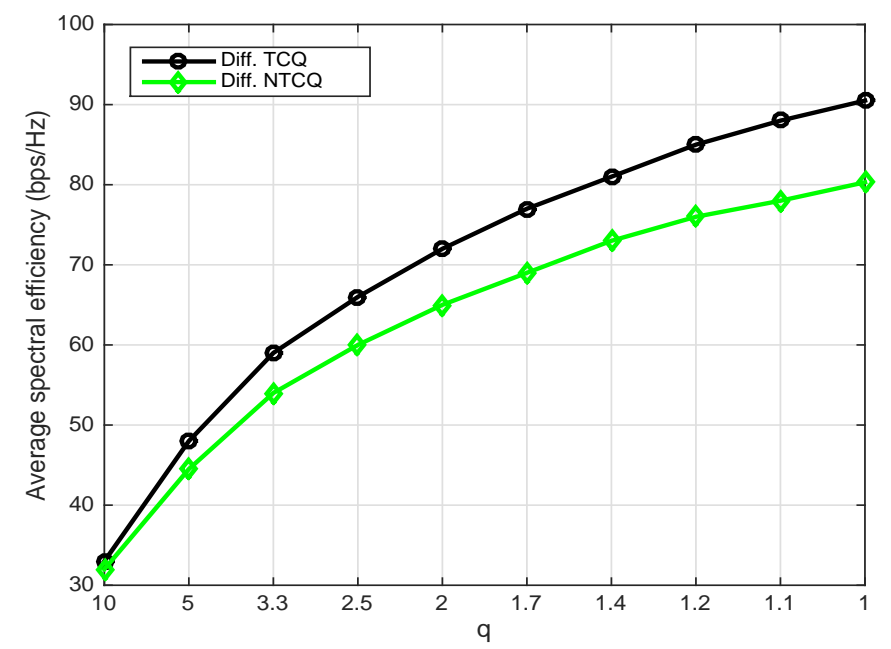

Figure 6.13: Average spectral efficiency for various $q=n_{t} / K$ values with QPSK constellation, $n_{t}=100$ and $\epsilon=0.9881(v=3 \mathrm{~km} / \mathrm{h})$.

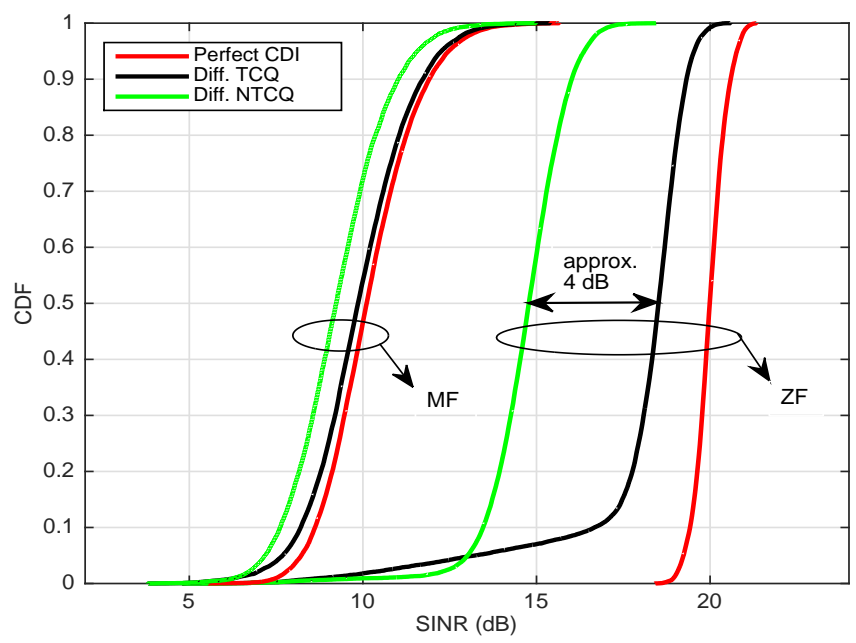

Figure 6.14: CDF of the SINR for an arbitrary user with QPSK constellation, $n_{t}=100$ and $\epsilon=0.9881(v=3 \mathrm{~km} / \mathrm{h})$.

both ZF and MF precoding schemes. 


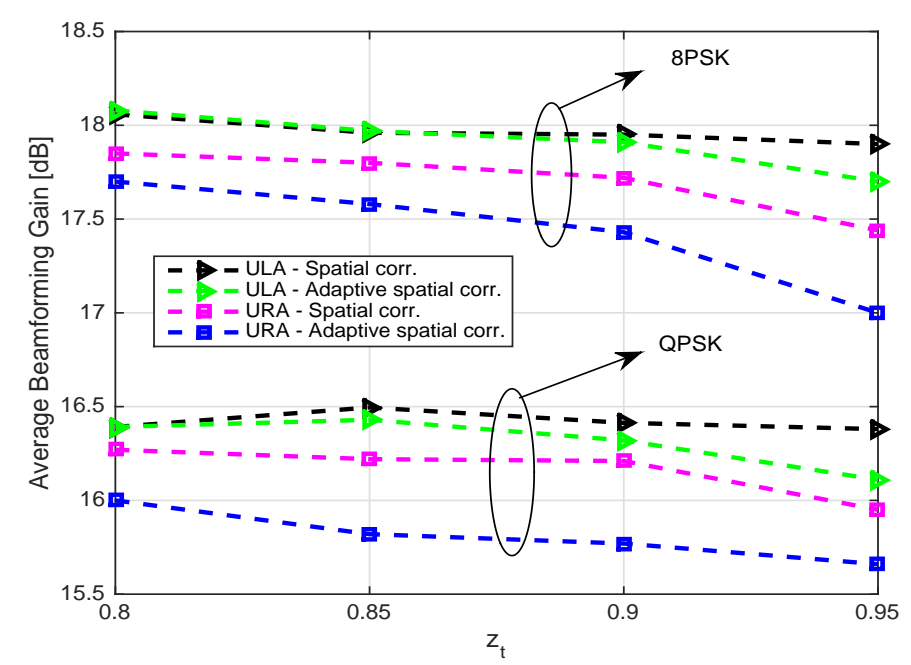

Figure 6.15: Average beamforming gain for different values of $z_{\mathrm{t}}$ with $n_{t}=100$.

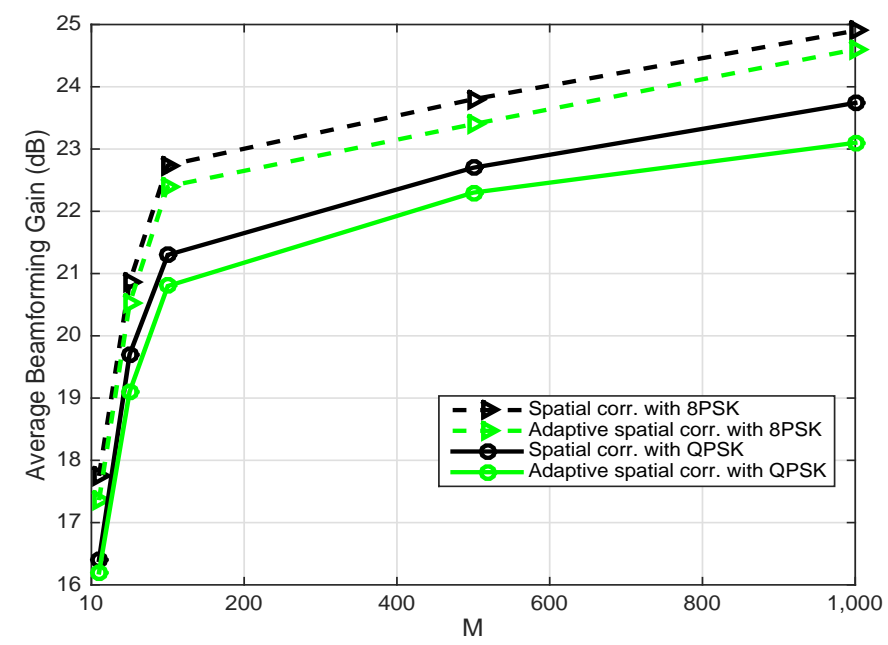

Figure 6.16: Average beamforming gain for different values of $n_{t}$ with $z_{\mathrm{t}}=0.99$.

\subsubsection{Spatially Correlated Channels}

For spatially correlated channels, we use the channel model in (6.32) and present the performance of the TCQ scheme for spatially correlated channels, discussed in Section 6.7, for MU massive MISO systems. We consider both ULA and URA antenna topologies as discussed in Section 6.7.

\section{Beamforming gain}

In Fig. 6.15, we compare the spatially correlated TCQ scheme and the adaptive TCQ technique presented [48], in highly correlated channel, such that $z_{\mathrm{t}} \rightarrow 1$. The comparison is performed in terms of the average beamforming gain for MISO systems given by $\mathbb{E}\left[|\hat{\mathbf{h}} \hat{\mathbf{q}}|^{2}\right]$, using QPSK and 8PSK constellations with 
ULA and URA. It is seen in Fig. 6.15 that the spatially correlated TCQ scheme performs slightly better than the adaptive NTCQ with both QPSK and 8PSK constellations. However, for not too high values of $z_{\mathrm{t}}$, the performance of both the schemes is equivalent.

It is important to mention that the TCQ scheme for spatially correlated channels (discussed in Section 6.7) does not require any knowledge (perfect or partial) of the spatial correlation matrix, $\mathbf{R}_{\mathrm{t}}$, at the transmitter. On the other hand, it is assumed in [48] that perfect knowledge of $\mathbf{R}_{\mathrm{t}}$ is available at the transmitter. It is also noted that the ULA provides higher beamforming gain compared to the URA. This is due to the fact that the URA induces higher spatial correlation in the channel than the ULA, thus resulting in reduced beamforming gain.

We plot average beamforming gain in Fig. 6.16 for different values of $n_{t}$ with fixed $z_{\mathrm{t}}=0.99$. At higher values of $n_{\mathrm{t}}$, the gain of the spatially correlated TCQ scheme is approximately $0.5 \mathrm{~dB}$ and $1 \mathrm{~dB}$ higher than the gain of the adaptive NTCQ scheme, with QPSK and 8PSK constellations, respectively.

\section{MU spectral efficiency performance}

The average spectral efficiency of the MU MISO system is shown in Fig. 6.17 for the spatially correlated TCQ scheme with $n_{t}=100$ and $z_{\mathrm{t}}=0.9$ and 0.99 .

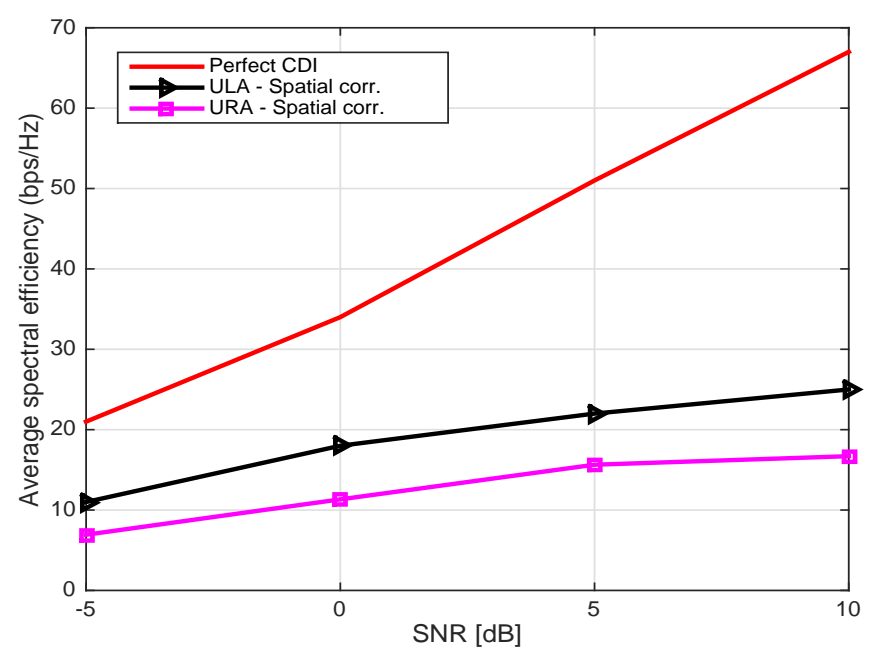

Figure 6.17: Average spectral efficiency against different SNR values for $n_{t}=100$ with ZF precoding.

ZF precoding is used to compute precoding vectors at the BS. It is noticed that the spatial correlation reduces the spectral efficiency of the TCQ based limited feedback MU MISO system. Also, interference due to the quantization process is a limiting factor that causes the spectral efficiency to saturate at high SNR values. Due to their being more adjacent antennas, the correlation in 
the URA is higher than that of the ULA and this results in a reduced spatial separation between users. Therefore, we note that the ULA provides significant improvement in the spectral efficiency compared to the URA. The dominance of ULA compared to other antenna topologies, in terms of the spectral efficiency, is also noticed in [123-125].

\subsubsection{WINNER II Channels}

We also evaluate the performance of the differential TCQ scheme (discussed in Section 6.6) in the standardized WINNER channel model given by (2.16). For the WINNER II channel, we use $n_{t}=100$ transmit antennas in a ULA setting with $0.5 \lambda$ spacing between them. We consider a UMa scenario with NLoS propagation. The carrier frequency is $2.5 \mathrm{GHz}$ and the velocity of the receiver is $v=1 \mathrm{~km} / \mathrm{h}$.

For the parameters discussed above, we generate WINNER II MISO channels and plot the average beamforming gain for massive MISO system with both QPSK and 8PSK constellations. As the WINNER II channel model is both temporally and spatially correlated, we rely on the differential TCQ scheme (discussed in Section 6.5) to keep track of the channel entries over time. Assuming that the velocity is perfectly estimated at the receiver, we compute the temporal correlation coefficient, $\epsilon$, as defined in FOGM channels (2.13). The result shown in Fig. 6.18 has a similar trend to that of the temporally correlated channel generated with the FOGM process shown in Fig. 6.9. This validates the performance of the differential TCQ scheme in real-world channels.

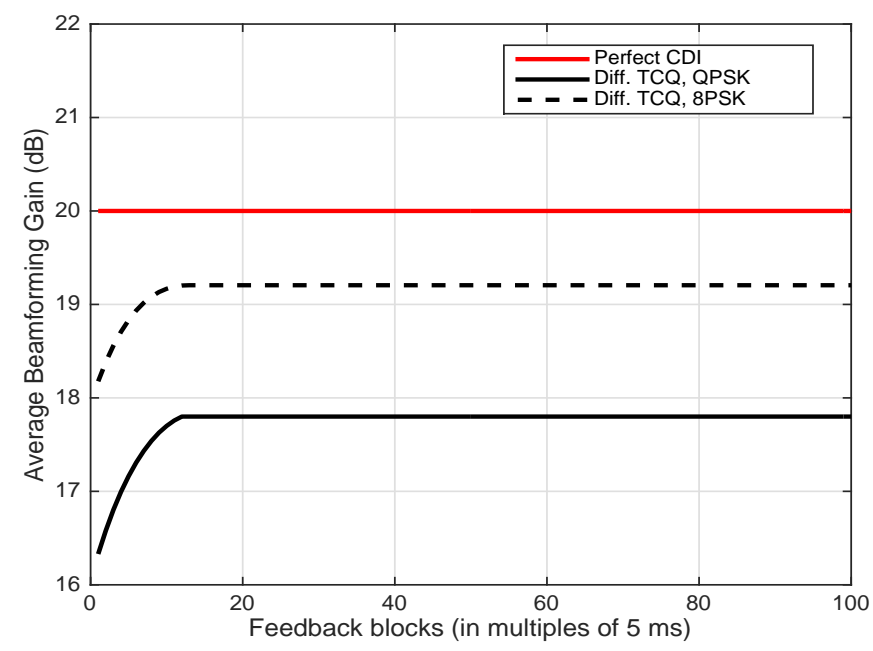

Figure 6.18: Average beamforming gain versus feedback intervals with $n_{t}=100$ for $\epsilon=0.9987(v=1 \mathrm{~km} / \mathrm{h})$.

It is evident from the numerical results that the TCQ schemes discussed in Section 6.6 and 6.7 for temporally and spatially correlated channels, respectively, 
yield better performance than existing NTCQ schemes [48]. For temporally correlated channels, we note that scaling and transforming the source constellation is helpful in tracking the slowly varying channel entries over time. It is due to this successful tracking that the differential TCQ scheme achieves high performance in terms of spectral efficiency and beamforming gain. On the other hand, the TCQ scheme for spatially correlated channels gives superior performance than the adaptive NTCQ scheme [48], as unlike the adaptive NTCQ scheme, it quantizes the correlated CDI. We also note that scaling the source constellation by $1 / \sqrt{n_{t}}$ yields better performance in high spatially correlated channels $\left(z_{t} \rightarrow 1\right)$.

\subsection{Summary}

In this chapter, we have discussed efficient TCQ schemes to quantize temporally and spatially correlated channels in a massive MISO setting. We evaluated performance using QPSK and 8PSK constellations and a single trellis decoder block at each user. In the differential TCQ, we transformed the source constellation at each stage in a trellis separately, such that the resulting constellation is centered around the previously selected constellation point. Consequently, 2D translation and scaling techniques were introduced to transform the source constellation. The scaling factor for the source constellation was also derived that exploits the temporal correlation present in the channel and scales the constellation accordingly for the given number of BS antennas and the temporal correlation coefficient.

One of the major advantages of the TCQ based limited feedback technique is that the selection and searching of the appropriate codeword becomes simpler using the Viterbi algorithm.

We have shown through simulations that the TCQ schemes discussed outperforms the NTCQ methods by improving the SINR and spectral efficiency results and reducing the feedback overhead by a small amount. 


\section{Coordinated Multicell MU MISO Systems}

Up to this point we have considered single-cell systems without out-of-cell interference. In this chapter, we investigate a mutlicell system where out-of-cell interference is considered. Unlike the previous chapter, we focus on conventional (small-scale) MU MISO systems with i.i.d. Rayleigh fading channels. We use a coordinated RZF precoding strategy where base stations installed at neighboring cells share interfering CSI. By considering this CSI while precoding the serving BS reduces interference at its users. Generally, in multicell systems the cell-edge users are more susceptible to the interference coming from the neighboring cells. Therefore, the main focus of this study is to reduce the interference at the celledge users through cell coordination. We derive expected SINR approximations for the coordinated RZF scheme with perfect CDI and RVQ codebook-based limited feedback quantized CDI. Later, we discuss the adaptive bit allocation scheme that minimizes the mean interference at the user, and present numerical performance results.

\subsection{Motivation and Related Work}

In cellular communication, the performance of the MU MIMO system is mainly assessed by the average spectral efficiency of the cell and by the data-rate at the cell-edge. For single-cell systems, one way to improve the cell-edge performance is by increasing the transmit power at the BS. However, for multicell systems doing this increases the level of interference to other cells. In practice the users close to the cell-edge are more susceptible to ICI. Consequently, their 
individual rate and the spectral efficiency of the cell degrades. Such a loss can be avoided by having coordination between base stations, where information is exchanged among the base stations via a backhaul link to suppress the ICI in the downlink $[29,30,126-130]$. An overview of the multicell systems is presented in [31].

Numerous studies have dealt with multicell MU MIMO systems with perfect CSI, as well as for limited feedback based quantized CSI. In [49], a limited feedback strategy for MU MISO multicell systems at high SNR is developed using RVQ codebooks [22]. Here, the idea is to design beamforming vectors using a generalized eigenvector approach. The multicell model considered in [49] is based on the Wyner model [131] and feedback bits are allocated adaptively among the serving and interfering channels by minimizing the mean rate loss of the system.

An adaptive bit allocation method which maximizes the spectral efficiency is proposed in [50] for limited feedback systems. Similarly, an adaptive feedback scheme for limited feedback MISO systems is developed in [128] with the ZF precoding scheme, where the adaptive bit allocation is realized by minimizing the expected spectral efficiency loss in multicell systems. The majority of the work discussed above has two common features, in that they all use RVQ codebooks and rely on solving optimization problems to adaptively allocate the bits to the serving and out-of-cell interfering channels.

An adaptive bit allocation scheme for joint processing (JP) MISO systems is presented in [51] with limited feedback. Here base stations not only exchange CSI but also share data of the users through backhaul links. The proposed work in [132] finds the precoder in each cell that maximizes a weighted spectral efficiency across the network. It has been shown to achieve twice the spectral efficiency performance compared to the conventional equal bit allocation scheme in a three cell network. More recently, a coordinated beamforming with an adaptive feedback bit allocation is proposed for MU heterogeneous networks in [52].

Despite the numerous studies on coordinated multicell systems, little attention was paid to coordinated RZF precoding until massive MIMO became popular $[133,134]$. Therefore, in this chapter, we investigate coordinated RZF precoding but for conventional (small-scale) multicell MU MISO systems. We develop a coordinated RZF precoding strategy [135], where base stations share out-of-cell interfering CSI to coordinate, such that interference at cell-edge users is suppressed. For this purpose, we adopt a coordination zone within each cell, as in [128]. The user in this coordination region is defined as a cell-edge user and it feeds back both serving and out-of-cell interfering channels to the serving BS. The serving BS shares the out-of-cell interfering CSI with the relevant interfering base stations. 
The main reason for sharing interfering channels is that the interfering BS considers these channels while designing precoding vectors to minimize the interference in the network. Although the idea of sharing out-of-cell interfering channels is presented in [128] using ZF precoding, in this study, we use RZF precoding which has been shown to be more effective than ZF in the low SNR regime [10]. We also derive expected SINR approximations for the coordinated RZF scheme with the perfect CDI and the RVQ codebook CDI at the BS. Furthermore, we develop an adaptive bit allocation scheme that distributes the bits to serving and out-of-cell interfering channels, such that the expected interference is minimized at the user.

\subsection{Downlink System Description}

Consider a multicell MU MISO system with $C$ coordinating cells consisting of a single BS each. Each BS has $n_{t}$ transmit antennas to simultaneously serve $K$ single antenna users in the cell. All the $C$ cells are interconnected via backhaul links. The backhaul is assumed to be error free and to have no delay. In this study, we assume that the total number of users in all the coordinating cells is equal to or less than the number of transmit antennas $n_{t}$ at each BS, such that, $C K \leq n_{t}{ }^{1}$. The channel vector of size $1 \times n_{t}$ between the $k^{\text {th }}$ user in the $c^{\text {th }}$ cell and the serving BS is given by $\sqrt{P_{k, c, c}} \mathbf{h}_{k, c, c}$, where $P_{k, c, c}$ denotes the instantaneous channel power of the user. Similarly, the interfering channel vector between the $k^{\text {th }}$ user in the $c^{\text {th }}$ cell and the $j^{\text {th }}$ interfering BS is denoted by $\sqrt{P_{k, c, j}} \mathbf{h}_{k, c, j}$, where $j \neq c$. The channel entries $\mathbf{h}_{k, c, c}$ and $\mathbf{h}_{k, c, j}$ are i.i.d. complex Gaussian with unit variance, i.e., $\mathcal{C N}(0,1)$. The downlink received signal at the $k^{\text {th }}$ user in the $c^{\text {th }}$ cell is given by

$$
\begin{aligned}
y_{k, c}= & \underbrace{\sqrt{\frac{P_{k, c, c}}{\gamma_{c}}} \mathbf{h}_{k, c, c} \mathbf{w}_{k, c} s_{k, c}}_{\text {desired signal }}+\underbrace{\sqrt{\frac{P_{k, c, c}}{\gamma_{c}}} \sum_{\substack{l=1 \\
l \neq k}}^{K} \mathbf{h}_{k, c, c} \mathbf{w}_{l, c} s_{l, c}}_{\text {inter-user interference }} \\
& +\underbrace{\sum_{\substack{j=1 \\
j \neq c}}^{C} \sqrt{\frac{P_{k, c, j}}{\gamma_{j}}} \mathbf{h}_{k, c, j} \sum_{q=1}^{K} \mathbf{w}_{q, j} s_{q, j}+\underbrace{n_{k, c},}_{\text {noise }}}_{\text {inter-cell interference }}
\end{aligned}
$$

where $\mathbf{w}_{k, c}$ is the non-normalized precoding vector for the $k^{\text {th }}$ user in the $c^{\text {th }}$ cell. $\gamma_{c}$ and $\gamma_{j}$ are the normalization parameter (to be discussed later in Section 7.3) for the $c^{\text {th }}$ and $j^{\text {th }}$ cell, respectively. $s_{k, c}$ and $n_{k, c}$ denote the data symbol and the

\footnotetext{
${ }^{1}$ In this chapter, we use RZF precoding and assume that $C K \leq n_{t}$, as at high SNR RZF boils down to ZF, given the fact that $n_{t}$ exceeds the total number of receive antennas in the system.
} 
noise for the $k^{\text {th }}$ user in the $c^{\text {th }}$ cell. The noise is assumed to be an i.i.d. complex Gaussian random variable with zero mean and variance $\sigma_{k}^{2}$, where without loss of generality we set $\sigma_{k}^{2}=1$. We assume that the data symbols are selected from the same constellation, where the symbol energy satisfies $\mathbb{E}\left[\left|s_{k, c}\right|^{2}\right]=1$.

The instantaneous received signal powers from the serving and interfering base stations at the $k^{\text {th }}$ user in the $c^{\text {th }}$ cell are modeled, respectively, by $(2.1)$ [136]

$$
P_{k, c, c}=P_{0}\left(\frac{R_{c}}{d_{k, c, c}}\right)^{a} \Phi_{k, c, c}
$$

and

$$
P_{k, c, j}=P_{0}\left(\frac{R_{c}}{d_{k, c, j}}\right)^{a} \Phi_{k, c, j}
$$

where $P_{0}$ is the received power at cell radius $R_{c}$ in the absence of shadowing, assumed to be equal for all cells, and $a$ is the path loss exponent. The shadowing, $\Phi_{k, c, c}$, between the $k^{\text {th }}$ user in the $c^{\text {th }}$ cell and $c^{\text {th }}$ BS, is modeled by a lognormal random variable, given by $\Phi_{k, c, c}=10^{\left(\eta_{k, c, c} \sigma_{S F} / 10\right)}$ [137], where $\sigma_{S F}$ is the shadowing standard deviation in $\mathrm{dB}$ and $\eta_{k, c, c}$ is a zero mean Gaussian random variable with unit variance. $d_{k, c, c}$ and $d_{k, c, j}$ represent the distances from the serving and the interfering base stations to the $k^{\text {th }}$ user in the $c^{\text {th }}$ cell, respectively.

\subsection{Coordinated RZF Precoding}

In this section, we explain the coordinated RZF precoding strategy for MU MISO systems. Each BS uses RZF precoding [77] to compute the precoding

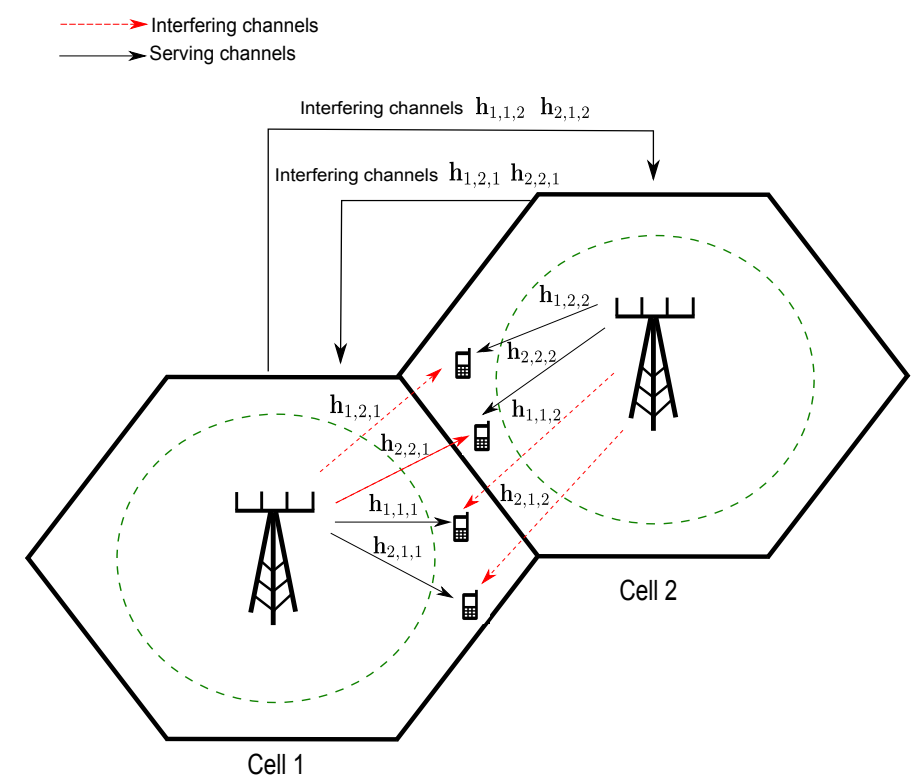

Figure 7.1: The system model for $C=2$ cells and $K=2$ cell-edge users. 
vectors for their respective cell users. In doing so, the serving BS applies RZF not only to the channels of serving users but also considers the interfering channels from it to the other cells. Doing so allows the serving BS to suppress the interference. All interfering channels and the serving channel at the $k^{\text {th }}$ user in the $c^{\text {th }}$ cell are determined using cell-specific pilots [138] and these channels are conveyed to the serving BS. We assume perfect channel estimation at the user and also assume that a sufficient number of orthogonal reference signals are available. The estimated interfering channels caused by the respective base stations are delivered to them via backhaul links. For example, the $c^{\text {th }}$ cell BS shares interfering channels with the $j^{\text {th }}$ cell BS and in return also receives interfering channels between it and $j^{\text {th }}$ cell users, through the backhaul link. The system model for $C=2$ cells and $K=2$ cell-edge users with serving and interfering channels is shown in Fig. 7.1, where interfering channels are represented by red-dashed arrows. With $C K \leq n_{t}$, the unnormalized RZF precoder $\mathbf{w}_{k, c}$, for the $k^{\text {th }}$ user in the $c^{\text {th }}$ cell is the $k^{\text {th }}$ column of $\mathbf{W}_{\text {rzf }, c}{ }^{2}$, given by [77]

$$
\mathbf{W}_{\mathrm{rzf}, c}=\mathbf{H}_{c}^{H}\left(\mathbf{H}_{c} \mathbf{H}_{c}^{H}+\zeta_{c} \mathbf{I}\right)^{-1}
$$

where $\mathbf{H}_{c}=\left[\mathbf{X}_{1}^{T} \mathbf{X}_{2}^{T} \ldots, \mathbf{X}_{c}^{T} \ldots \mathbf{X}_{C}^{T}\right]^{T}$ is a $C K \times n_{t}$ concatenated channel matrix with $\mathbf{X}_{1}=\left[\mathbf{h}_{1,1, c}^{T} \ldots \mathbf{h}_{K, 1, c}^{T}\right]^{T}$ and $\mathbf{X}_{c}=\left[\mathbf{h}_{1, c, c}^{T} \ldots \mathbf{h}_{K, c, c}^{T}\right]^{T}$. The resulting precoder matrix is normalized by the normalization parameter, such that $\mathbf{W}_{\text {rzf }, c} / \sqrt{\gamma_{c}}$, where $\gamma_{c}=\left\|\mathbf{W}_{\mathrm{rzf}, c}\right\|_{F}^{2} / n_{t}$. This normalization is necessary to achieve $\mathbb{E}\left[\left\|\mathbf{w}_{k, c}\right\|^{2}\right]=1$. The regularization parameter for the $c^{\text {th }}$ BS is denoted by $\zeta_{c}$. The choice of regularization parameter is discussed in Section 7.5. When perfect CDI and CQI are available at the BS, the SINR expression for the $k^{\text {th }}$ user in the $c^{\text {th }}$ cell using $(7.1)$ can be written as $[134,139]$

$$
\operatorname{SINR}_{k, c}=\frac{\frac{P_{k, c, c}}{\gamma_{c}}\left|\mathbf{h}_{k, c, c} \mathbf{w}_{k, c}\right|^{2}}{1+\frac{P_{k, c, c}}{\gamma_{c}} \sum_{\substack{l=1 \\ l \neq k}}^{K}\left|\mathbf{h}_{k, c, c} \mathbf{w}_{l, c}\right|^{2}+\sum_{\substack{j=1 \\ j \neq c}}^{C} \frac{P_{k, c, j}}{\gamma_{j}} \sum_{q=1}^{K}\left|\mathbf{h}_{k, c, j} \mathbf{w}_{q, j}\right|^{2}}
$$

\subsubsection{Expected SINR with Perfect CDI}

In this section, we derive an approximation to the expected SINR with perfect CDI for the coordinated RZF scheme discussed in Section 7.3. We can take the expectation of the SINR in (7.5) and approximate it by using the result in [119],

\footnotetext{
${ }^{2}$ The true serving channel at the $k^{\text {th }}$ user in the $c^{\text {th }}$ cell is given by, $\sqrt{P_{k, c, c}} \mathbf{h}_{k, c, c}$, however in this study, like $[134,139]$, we do not consider path-loss and shadowing while computing the precoding matrix, $\mathbf{W}_{\mathrm{rzf}, c}$, in (7.4).
} 
such that

$$
\begin{aligned}
& \mathbb{E}\left[\mathrm{SINR}_{k, c}\right] \approx \\
& \frac{\frac{P_{k, c, c}}{\bar{\gamma}_{c}} \mathbb{E}\left[\left|\mathbf{h}_{k, c, c} \mathbf{w}_{k, c}\right|^{2}\right]}{1+\frac{P_{k, c, c}}{\bar{\gamma}_{c}} \sum_{\substack{l=1 \\
l \neq k}}^{K} \mathbb{E}\left[\left|\mathbf{h}_{k, c, c} \mathbf{w}_{l, c}\right|^{2}\right]+\sum_{\substack{j=1 \\
j \neq c}}^{C} \frac{P_{k, c, j}}{\bar{\gamma}_{j}} \sum_{q=1}^{K} \mathbb{E}\left[\left|\mathbf{h}_{k, c, j} \mathbf{w}_{q, j}\right|^{2}\right]} .
\end{aligned}
$$

where $\bar{\gamma}_{c}=\mathbb{E}\left[\gamma_{c}\right]$ and $\bar{\gamma}_{j}=\mathbb{E}\left[\gamma_{j}\right]$. Note that the numerator and the denominator are dependent as they share some random variables in common. This complicates the calculation of the mean SINR and we therefore employ the SINR approximation approach given in [119]. This approximation has been shown to get tighter as $M$ grows large while also providing good accuracy for smaller $M$ values. We now evaluate (7.6) by computing the expected signal power and the expected interference power in (7.6) for the case $C K=n_{t}$, where the maximum number of users in the network are supported. However, the following analysis can also be applied to the scenarios where $C K<n_{t}$. To compute the expectations, we use the approach presented in [77], and moreover, we evaluate the expectation over the eigenvalues of $\mathbf{H}_{c} \mathbf{H}_{c}^{H}$, which has not been investigated in [77].

Expected signal power: The expected signal power in (7.6) is

$$
S_{k, c}=\frac{P_{k, c, c}}{\bar{\gamma}_{c}} \mathbb{E}\left[\left|\mathbf{h}_{k, c, c} \mathbf{W}_{k, c}\right|^{2}\right]
$$

Using the eigenvalue decomposition, $\mathbf{H}_{c} \mathbf{H}_{c}^{H}=\mathbf{Q} \Lambda \mathbf{Q}^{H}$, the expectation in (7.7), denoted by $\delta_{k, c}$, is written as [77]

$$
\delta_{k, c}=\mathbb{E}\left[\left|\mathbf{h}_{k, c, c} \mathbf{w}_{k, c}\right|^{2}\right]=\mathbb{E}\left[\left(\sum_{n=1}^{n_{t}} \frac{\lambda_{n}}{\lambda_{n}+\zeta_{c}}\left|q_{k, n}\right|^{2}\right)^{2}\right],
$$

where $\lambda_{n}$ is the $n^{\text {th }}$ eigenvalue corresponding to the $n^{\text {th }}$ diagonal entry of $\Lambda$. The quantity $q_{k, n}$ denotes the entry of $\mathbf{Q}$ corresponding to the $k^{\text {th }}$ row and $n^{\text {th }}$ column. Using [77, Appendix A], the mean over the entries of $\mathbf{Q}$ yields

$$
\delta_{k, c}=\frac{1}{n_{t}\left(n_{t}+1\right)}\left(\mathbb{E}_{\lambda}\left[\left(\sum_{n=1}^{n_{t}} \frac{\lambda_{n}}{\lambda_{n}+\zeta_{c}}\right)^{2}\right]+\mathbb{E}_{\lambda}\left[\sum_{n=1}^{n_{t}}\left(\frac{\lambda_{n}}{\lambda_{n}+\zeta_{c}}\right)^{2}\right]\right)
$$

The value of $\bar{\gamma}_{c}$ is given by

$$
\bar{\gamma}_{c}=\frac{1}{n_{t}} \mathbb{E}\left[\left\|\mathbf{W}_{\mathrm{rzf}, c}\right\|_{F}^{2}\right]=\frac{1}{n_{t}} \mathbb{E}_{\lambda}\left[\sum_{n=1}^{n_{t}} \frac{\lambda_{n}}{\left(\lambda_{n}+\zeta_{c}\right)^{2}}\right] .
$$

The expectation of the terms in (7.9) and (7.10), with respect to the eigen- 
values, are given in Result 1 and Result 2 .

Result 1: When the entries of an $n_{t} \times n_{t}$ matrix $\mathbf{H}$ are i.i.d. $\mathcal{C N}(0,1)$, then the expected value of $\sum_{n=1}^{n_{t}} \frac{\left(\lambda_{n}\right)^{t}}{\left(\lambda_{n}+\zeta_{c}\right)^{2}}$, where $\lambda_{n}$ is the $n^{\text {th }}$ eigenvalue of $\mathbf{H H}^{H}$ (uncorrelated central Wishart matrix), with respect to $\lambda_{n} \forall n$, is given by

$$
\begin{aligned}
\mathrm{D}_{c}^{(t)}= & \mathbb{E}\left[\sum_{n=1}^{n_{t}} \frac{\left(\lambda_{n}\right)^{t}}{\left(\lambda_{n}+\zeta_{c}\right)^{2}}\right] \\
= & \sum_{i=1}^{n_{t}} \sum_{j=0}^{i-1} \sum_{l=0}^{i-1}(-1)^{j+l}\left(\begin{array}{c}
i-1 \\
i-1-j
\end{array}\right)\left(\begin{array}{c}
i-1 \\
i-1-l
\end{array}\right) \frac{1}{j ! l !} \sum_{s=0}^{t+j+l}\left(\begin{array}{c}
t+j+l \\
s
\end{array}\right) \\
& \left(-\zeta_{c}\right)^{t+j+l-s} \mathrm{e}^{\zeta_{c}} \int_{\zeta_{c}}^{\infty} v^{s-2} \mathrm{e}^{-v} d v .
\end{aligned}
$$

Proof: See Appendix A.

Result 2: When the entries of an $n_{t} \times n_{t}$ matrix $\mathbf{H}$ are i.i.d. $\mathcal{C N}(0,1)$, then the expected value of $\left(\sum_{n=1}^{n_{t}} \frac{\lambda_{n}}{\lambda_{n}+\zeta_{c}}\right)^{2}$, where $\lambda_{n}$ is the $n^{\text {th }}$ eigenvalue of $\mathbf{H H}^{H}$ (uncorrelated central Wishart matrix), with respect to $\lambda_{n} \forall n$, is given by

$$
\begin{aligned}
\mathrm{F}_{c}= & \mathbb{E}\left[\left(\sum_{n=1}^{n_{t}} \frac{\lambda_{n}}{\lambda_{n}+\zeta_{c}}\right)^{2}\right]=\mathrm{D}_{c}^{(2)}+\sum_{i=1}^{n_{t}} \sum_{j=1, j \neq i}^{n_{t}} \\
& \left(\sum_{r=0}^{i-1} \sum_{s=0}^{i-1}(-1)^{r+s}\left(\begin{array}{c}
i-1 \\
i-1-r
\end{array}\right)\left(\begin{array}{c}
i-1 \\
i-1-s
\end{array}\right) \frac{1}{r ! s !} \sum_{b=0}^{1+r+s}\left(\begin{array}{c}
1+r+s \\
b
\end{array}\right)\left(-\zeta_{c}\right)^{1+r+s-b}\right. \\
& \left.\mathrm{e}^{\zeta_{c}} \int_{\zeta_{c}}^{\infty} v^{b-1} \mathrm{e}^{-v} d v\right)^{2}-\left(\sum_{r=0}^{i-1} \sum_{s=0}^{j-1}(-1)^{r+s}\left(\begin{array}{c}
i-1 \\
i-1-r
\end{array}\right)\left(\begin{array}{c}
j-1 \\
j-1-s
\end{array}\right) \frac{1}{r ! s !}\right. \\
& \left.\sum_{b=0}^{1+r+s}\left(\begin{array}{c}
1+r+s \\
b
\end{array}\right)\left(-\zeta_{c}\right)^{1+r+s-b} \mathrm{e}^{\zeta_{c}} \int_{\zeta_{c}}^{\infty} v^{b-1} \mathrm{e}^{-v} d v\right)^{2}
\end{aligned}
$$

where $\mathrm{D}_{c}^{(2)}$ is obtained from (7.11).

Proof: See Appendix B.

Using Result 1 and 2, we can write (7.9) and (7.10) as

$$
\delta_{k, c}=\frac{\mathrm{F}_{c}+\mathrm{D}_{c}^{(2)}}{n_{t}\left(n_{t}+1\right)}
$$

and

$$
\bar{\gamma}_{c}=\frac{\mathrm{D}_{c}^{(1)}}{n_{t}} .
$$

Therefore, the expected signal power (7.7) can be written as

$$
S_{k, c}=\frac{P_{k, c, c}}{\bar{\gamma}_{c}} \delta_{k, c}
$$


Expected interference power: The expected interference power in (7.6) is given by

$$
I_{k, c}=\frac{P_{k, c, c}}{\bar{\gamma}_{c}} \sum_{\substack{l=1 \\ l \neq k}}^{K} \mathbb{E}\left[\left|\mathbf{h}_{k, c, c} \mathbf{w}_{l, c}\right|^{2}\right]+\sum_{\substack{j=1 \\ j \neq c}}^{C} \frac{P_{k, c, j}}{\bar{\gamma}_{j}} \sum_{q=1}^{K} \mathbb{E}\left[\left|\mathbf{h}_{k, c, j} \mathbf{w}_{q, j}\right|^{2}\right]
$$

In order to evaluate (7.16), we observe that

$$
\psi \triangleq \mathbb{E}\left[\left|\mathbf{h}_{k, c, c} \mathbf{w}_{l, c}\right|^{2}\right]=\mathbb{E}\left[\left|\mathbf{h}_{k, c, j} \mathbf{w}_{q, j}\right|^{2}\right], \forall l, j, q
$$

Hence,

$$
I_{k, c}=\frac{P_{k, c, c}}{\bar{\gamma}_{c}}(K-1) \psi+\sum_{\substack{j=1 \\ j \neq c}}^{C} \frac{P_{k, c, j}}{\bar{\gamma}_{j}} K \psi
$$

Now $\psi$ can be found from $\psi_{\text {sum }} /\left(n_{t}-1\right)$, where

$$
\psi_{\text {sum }}=\sum_{\substack{l=1 \\ l \neq k}}^{K} \mathbb{E}\left[\left|\mathbf{h}_{k, c, c} \mathbf{w}_{l, c}\right|^{2}\right]+\sum_{\substack{j=1 \\ j \neq c}}^{C} \sum_{q=1}^{K} \mathbb{E}\left[\left|\mathbf{h}_{k, c, j} \mathbf{w}_{q, j}\right|^{2}\right] .
$$

In order to compute $\psi_{\text {sum }}$, we note that $(7.19)$ is the expected interference in the absence of the powers and normalization parameters. Hence, (7.19) is given by the difference between the expected total received power and the expected signal power [77], again neglecting the power and normalization terms. Hence, we can write $(7.19)$ as

$$
\begin{aligned}
\psi_{\text {sum }} & =\xi_{c}-\delta_{k, c} \\
& =\left[\frac{\mathrm{D}_{c}^{(2)}}{n_{t}}-\frac{\mathrm{F}_{c}+\mathrm{D}_{c}^{(2)}}{n_{t}\left(n_{t}+1\right)}\right],
\end{aligned}
$$

where $\xi_{c}$ is the expected total received signal (desired and interference) power at the user in the $c^{\text {th }}$ cell, given by $\xi_{c}=\mathbb{E}\left[\left\|\mathbf{H}_{c} \mathbf{W}_{\mathrm{rzf}, c}\right\|_{F}^{2}\right] / n_{t}=\mathrm{D}_{c}^{(2)} / n_{t}$.

Expected SINR with perfect CDI: We can now write the expected SINR in (7.6) in terms of $\delta_{k, c}, \psi_{c}, \psi_{j}, \bar{\gamma}_{c}$ and $\bar{\gamma}_{j}$ as

$$
\mathbb{E}\left[\operatorname{SINR}_{k, c}\right] \approx \frac{\frac{P_{k, c, c}}{\bar{\gamma}_{c}} \delta_{k, c}}{1+\frac{P_{k, c, c}}{\bar{\gamma}_{c}}(K-1) \psi_{c}+\sum_{\substack{j=1 \\ j \neq c}}^{C} \frac{P_{k, c, j}}{\bar{\gamma}_{j}} K \psi_{j}} .
$$

Having derived the expected SINR approximation for the perfect CDI case, we now investigate coordinated RZF precoding with limited feedback MU MISO systems. 


\subsubsection{Expected SINR with RVQ CDI}

In this section, we discuss coordinated RZF precoding with limited feedback. Like the perfect CDI case, here we give the derivation details for the expected SINR approximation. In limited feedback multiple antenna systems, the user quantizes the estimated channel (we assume perfect estimation) using a codebook. The quantized vector of the channel between the $k^{\text {th }}$ user in the $c^{\text {th }}$ cell and the $c^{\text {th }}$ BS is denoted by $\mathbf{x}_{k, c, c}$. We consider RVQ codebooks and $B$ is the total number of feedback bits ${ }^{3}$. Each user quantizes serving and out-of-cell interfering channels, and so $B=\sum_{i=1}^{C} B_{k, c, i}$. The feedback bits are sent to the $\mathrm{BS}$ via a low-rate feedback link. In this case, the perfect concatenated channel matrix for the $c^{\text {th }}$ BS can be modeled as $[140,141]$

$$
\mathbf{H}_{c}=\mathbf{T}_{c}+\mathbf{E}_{c}
$$

where $\mathbf{H}_{c} \sim \mathcal{C N}(0,1)$ and $\mathbf{E}_{c}=\left[\mathbf{G}_{1}^{T} \mathbf{G}_{2}^{T} \ldots, \mathbf{G}_{c}^{T} \ldots, \mathbf{G}_{C}^{T}\right]^{T}$ is a $C K \times n_{t}$ concatenated quantization error matrix, such that $\mathbf{G}_{1}=\left[\mathbf{e}_{1,1, c}^{T} \ldots \mathbf{e}_{K, 1, c}^{T}\right]^{T}$. The quantization error matrix, $\mathbf{E}_{c}$, is assumed to have independent complex Gaussian elements $[140,141]$ such that the quantization error vector between the $k^{\text {th }}$ user in the $c^{\text {th }}$ cell and the $c^{\text {th }} \mathrm{BS}$, denoted by $\mathbf{e}_{k, c, c}$, is given by $\mathbf{e}_{k, c, c} \sim \mathcal{C} \mathcal{N}\left(0, \varrho_{k, c, c}^{2} \mathbf{I}\right)$. Using an upper bound on the quantization error for RVQ codebooks in terms of squared chordal distance given in [27], denoting by $B_{k, c, c}$ the number of bits used to quantize the channel between the $k^{\text {th }}$ user in the $c^{\text {th }}$ cell and the $c^{\text {th }}$ BS, we have, $\varrho_{k, c, c}^{2} \leq 2^{\frac{-B_{k, c, c}}{n_{t}-1}}$. In our model, we assume the worst case scenario where $\varrho_{k, c, c}^{2} \sim 2^{\frac{-B_{k, c, c}}{M-1}}$. Similarly, the entries of the quantized concatenated channel ma$\operatorname{trix}, \mathbf{T}_{c}=\left[\begin{array}{lll}\tilde{\mathbf{G}}_{1}^{T} & \tilde{\mathbf{G}}_{2}^{T} \ldots \tilde{\mathbf{G}}_{c}^{T} \ldots \tilde{\mathbf{G}}_{C}^{T}\end{array}\right]^{T}$ where $\tilde{\mathbf{G}}_{1}=\left[\mathbf{x}_{1,1, c}^{T} \ldots \mathbf{x}_{K, 1, c}^{T}\right]^{T}$, are assumed to be independent and complex Gaussian, such that $\mathbf{x}_{k, c, c} \sim \mathcal{C N}\left(0,\left(1-\varrho_{k, c, c}^{2}\right) \mathbf{I}\right)$. The unnormalized precoding vector of the $k^{\text {th }}$ user in the $c^{\text {th }}$ cell, $\hat{\mathbf{w}}_{k, c}$, is the $k^{\text {th }}$ column of the matrix $\hat{\mathbf{W}}_{\text {rzf }, c}$, given by

$$
\hat{\mathbf{W}}_{\mathrm{rzf}, c}=\tilde{\mathbf{T}}_{c}^{H}\left(\tilde{\mathbf{T}}_{c} \tilde{\mathbf{T}}_{c}^{H}+\zeta_{c} \mathbf{I}\right)^{-1}
$$

where $\tilde{\mathbf{T}}_{c}=\left[\begin{array}{lll}\tilde{\mathbf{X}}_{1}^{T} & \tilde{\mathbf{X}}_{2}^{T} \ldots \tilde{\mathbf{X}}_{c}^{T} \ldots \tilde{\mathbf{X}}_{C}^{T}\end{array}\right]^{T}$ is a $C K \times n_{t}$ concatenated matrix with $\tilde{\mathbf{X}}_{1}=\left[\tilde{\mathbf{x}}_{1,1, c}^{T} \ldots \tilde{\mathbf{x}}_{K, 1, c}^{T}\right]^{T}$, and $\tilde{\mathbf{x}}_{k, c, c}=\mathbf{x}_{k, c, c} / \sqrt{1-\varrho_{k, c, c}^{2}}$ in order to have $\tilde{\mathbf{T}}_{c} \sim$ $\mathcal{C N}(0,1)$. In order to satisfy the power constraint, the precoding matrix is normalized by the parameter, $\hat{\gamma}_{c}$, such that, $\overline{\mathbf{W}}_{\text {rzf }, c}=\hat{\mathbf{W}}_{\text {rzf }, c} / \sqrt{\hat{\gamma}_{c}}$, where $\hat{\gamma}_{c}=$ $\left\|\hat{\mathbf{W}}_{\text {rzf, }, ~}\right\|_{F}^{2} / n_{t}$. On the downlink, the received signal for the $k^{\text {th }}$ user in the $c^{\text {th }}$

\footnotetext{
${ }^{3}$ We only study the effects of CDI quantization errors as in $[45,46,128]$. The quantization of CQI is out of the scope of this study.
} 
cell is given by, similar to (7.1), as

$$
\begin{aligned}
\hat{y}_{k, c} & =\sqrt{\frac{P_{k, c, c}}{\hat{\gamma}_{c}}}\left(\mathbf{x}_{k, c, c}+\mathbf{e}_{k, c, c}\right) \hat{\mathbf{w}}_{k, c} s_{k, c}+\sqrt{\frac{P_{k, c, c}}{\hat{\gamma}_{c}}} \sum_{\substack{l=1 \\
l \neq k}}^{K}\left(\mathbf{x}_{k, c, c}+\mathbf{e}_{k, c, c}\right) \hat{\mathbf{w}}_{l, c} s_{l, c} \\
& +\sum_{\substack{j=1 \\
j \neq c}}^{C} \sqrt{\frac{P_{k, c, j}}{\hat{\gamma}_{j}}}\left(\mathbf{x}_{k, c, j}+\mathbf{e}_{k, c, j}\right) \sum_{q=1}^{K} \hat{\mathbf{w}}_{q, j} s_{q, j}+n_{k, c} .
\end{aligned}
$$

In the case of RVQ codebooks, the expected SINR at the $k^{\text {th }}$ user in the $c^{\text {th }}$ cell, denoted by $\mathbb{E}\left[\widetilde{\operatorname{SINR}}_{k, c}\right]$, is approximated by [119], similar to (7.6), as

$\mathbb{E}\left[\widetilde{\operatorname{SINR}}_{k, c}\right] \approx$

$$
\frac{\frac{P_{k, c, c}}{\bar{\gamma}_{c}} \mathbb{E}\left[\left|\left(\mathbf{x}_{k, c, c}+\mathbf{e}_{k, c, c}\right) \hat{\mathbf{w}}_{k, c}\right|^{2}\right]}{1+\frac{P_{k, c, c}}{\bar{\gamma}_{c}} \sum_{\substack{l=1 \\ l \neq k}}^{K} \mathbb{E}\left[\left|\left(\mathbf{x}_{k, c, c}+\mathbf{e}_{k, c, c}\right) \hat{\mathbf{w}}_{l, c}\right|^{2}\right]+\sum_{\substack{j=1 \\ j \neq c}}^{C} \frac{P_{k, c, j}}{\bar{\gamma}_{j}} \sum_{q=1}^{K} \mathbb{E}\left[\left|\left(\mathbf{x}_{k, c, j}+\mathbf{e}_{k, c, j}\right) \hat{\mathbf{w}}_{q, j}\right|^{2}\right]},
$$

where $\bar{\gamma}_{c}=\mathbb{E}\left[\hat{\gamma}_{c}\right]$ and $\bar{\gamma}_{j}=\mathbb{E}\left[\hat{\gamma}_{j}\right]$. The tightness of (7.25) is discussed in Section 7.6. As for the perfect CDI case, we compute the various expectation terms in (7.25) individually, following a similar procedure for the $C K=n_{t}$ case.

Expected signal power: The expectation of the signal power at the $k^{\text {th }}$ user in the $c^{\text {th }}$ cell in (7.25) is given by

$$
S_{k, c}^{\prime}=\frac{P_{k, c, c}}{\bar{\gamma}_{c}} \mathbb{E}\left[\left|\left(\mathbf{x}_{k, c, c}+\mathbf{e}_{k, c, c}\right) \hat{\mathbf{w}}_{k, c}\right|^{2}\right] .
$$

We can write

$$
\begin{aligned}
\mathbb{E}\left[\left|\left(\mathbf{x}_{k, c, c}+\mathbf{e}_{k, c, c}\right) \hat{\mathbf{w}}_{k, c}\right|^{2}\right] & =\mathbb{E}\left[\left|\mathbf{x}_{k, c, c} \hat{\mathbf{w}}_{k, c}+\mathbf{e}_{k, c, c} \hat{\mathbf{w}}_{k, c}\right|^{2}\right] \\
& \stackrel{(a)}{=} \mathbb{E}\left[\left|\mathbf{x}_{k, c, c} \hat{\mathbf{w}}_{k, c}\right|^{2}\right]+\mathbb{E}\left[\left|\mathbf{e}_{k, c, c} \hat{\mathbf{w}}_{k, c}\right|^{2}\right] \\
& \stackrel{(b)}{=}\left(1-2^{\frac{-B_{k, c, c}}{n_{t}-1}}\right) \mathbb{E}\left[\left|\tilde{\mathbf{x}}_{k, c, c} \hat{\mathbf{w}}_{k, c}\right|^{2}\right]+\mathbb{E}\left[\left|\mathbf{e}_{k, c, c} \hat{\mathbf{w}}_{k, c}\right|^{2}\right],
\end{aligned}
$$

where $(a)$ follows because the expected value of the cross product terms is zero and for $(b)$ we use $\mathbf{x}_{k, c, c}=\sqrt{1-2^{\frac{-B_{k, c, c}}{n_{t}-1}}} \tilde{\mathbf{x}}_{k, c, c}$. Denoting the eigenvalue values of $\tilde{\mathbf{H}}_{c} \tilde{\mathbf{H}}_{c}=\tilde{\mathbf{Q}} \tilde{\mathbf{\Lambda}} \tilde{\mathbf{Q}}^{H}$ by $\tilde{\lambda}_{1}, \tilde{\lambda}_{2}, \ldots \tilde{\lambda}_{n t}$, and using the same approach as (7.7), we can express the expectation in the first term in (7.28) as 


$$
\begin{aligned}
\mathbb{E}\left[\left|\tilde{\mathbf{x}}_{k, c, c} \hat{\mathbf{w}}_{k, c}\right|^{2}\right] & =\frac{1}{n_{t}\left(n_{t}+1\right)}\left(\mathbb{E}_{\tilde{\lambda}}\left[\left(\sum_{n=1}^{n_{t}} \frac{\tilde{\lambda}_{n}}{\tilde{\lambda}_{n}+\zeta_{c}}\right)^{2}\right]+\mathbb{E}_{\tilde{\lambda}}\left[\sum_{n=1}^{n_{t}}\left(\frac{\tilde{\lambda}_{n}}{\tilde{\lambda}_{n}+\zeta_{c}}\right)^{2}\right]\right) \\
& =\delta_{k, c} .
\end{aligned}
$$

Also, we have

$$
\begin{aligned}
\mathbb{E}\left[\left|\mathbf{e}_{k, c, c} \hat{\mathbf{w}}_{k, c}\right|^{2}\right] & =\mathbb{E}\left[\left|\mathbf{e}_{k, c, c}\right|^{2}\right] \frac{\mathbb{E}\left[\left\|\hat{\mathbf{W}}_{c}\right\|_{F}^{2}\right]}{n_{t}} \\
& =\left(2^{\frac{-B_{k, c, c}}{n_{t}-1}}\right) \bar{\gamma}_{c} .
\end{aligned}
$$

Therefore, one obtains

$$
\mathbb{E}\left[\left|\left(\mathbf{x}_{k, c, c}+\mathbf{e}_{k, c, c}\right) \hat{\mathbf{w}}_{k, c}\right|^{2}\right]=\left(1-2^{\frac{-B_{k, c, c}}{n_{t}-1}}\right) \delta_{k, c}+\left(2^{\frac{-B_{k, c, c}}{n_{t}-1}}\right) \bar{\gamma}_{c}
$$

where

$$
\bar{\gamma}_{c}=\frac{1}{n_{t}} \mathbb{E}_{\tilde{\lambda}_{n}}\left[\sum_{n=1}^{n_{t}} \frac{\tilde{\lambda}_{n}}{\left(\tilde{\lambda}_{n}+\zeta_{c}\right)^{2}}\right] .
$$

Using Result 1 and Result 2, $\bar{\gamma}_{c}$ and $\delta_{k, c}$ can be written as

$$
\bar{\gamma}_{c}=\frac{\mathrm{D}_{c}^{(1)}}{n_{t}}
$$

and

$$
\delta_{k, c}=\frac{\mathrm{F}_{c}+\mathrm{D}_{c}^{(2)}}{n_{t}\left(n_{t}+1\right)},
$$

and thus the expected signal power in (7.26) can be expressed as

$$
S_{k, c}^{\prime}=\frac{P_{k, c, c}}{\bar{\gamma}_{c}}\left[\left(1-2^{\frac{-B_{k, c, c}}{n_{t}-1}}\right) \delta_{k, c}+\left(2^{\frac{-B_{k, c, c}}{n_{t}-1}}\right) \bar{\gamma}_{c}\right]
$$

Expected interference power: Now we can solve for the expected interference in (7.25) given by

$$
I_{k, c}^{\prime}=\frac{P_{k, c, c}}{\bar{\gamma}_{c}} \sum_{\substack{l=1 \\ l \neq k}}^{K} \mathbb{E}\left[\left|\left(\mathbf{x}_{k, c, c}+\mathbf{e}_{k, c, c}\right) \hat{\mathbf{w}}_{l, c}\right|^{2}\right]+\sum_{\substack{j=1 \\ j \neq c}}^{C} \frac{P_{k, c, j}}{\bar{\gamma}_{j}} \sum_{q=1}^{K} \mathbb{E}\left[\left|\left(\mathbf{x}_{k, c, j}+\mathbf{e}_{k, c, j}\right) \hat{\mathbf{w}}_{q, j}\right|^{2}\right] .
$$

Similar to the perfect CDI case, in order to find the expected interference terms 
in (7.36) (without pathloss and shadowing) at the $k^{\text {th }}$ user in the $c^{\text {th }}$ cell, we subtract the expected signal power given in (7.31) from the total expected received power, giving

$$
\begin{aligned}
\tilde{\psi}_{k, c} & =(\underbrace{\mathbb{E}\left[\left|\hat{\mathbf{e}}_{k, c, c} \hat{\mathbf{W}}_{\mathrm{rzf}, c}\right|_{F}^{2}\right]+\mathbb{E}\left[\left|\mathbf{x}_{k, c, c} \hat{\mathbf{W}}_{\mathrm{rzf}, c}\right|_{F}^{2}\right]}_{\text {total power at the user }}-\left[\left(1-2^{\frac{-B_{k, c, c}}{n_{t}-1}}\right) \delta_{k, c}+\left(2^{\frac{-B_{k, c, c}}{n_{t}-1}}\right) \bar{\gamma}_{c}\right]) \\
& =\left(\bar{\gamma}_{c} n_{t} 2^{\frac{-B_{k, c, c}}{n_{t}-1}}+\left(1-2^{\frac{-B_{k, c, c}}{n_{t}-1}}\right) \frac{\mathbb{E}\left[\left|\tilde{\mathbf{T}}_{c} \hat{\mathbf{W}}_{\mathrm{rzf}, c}\right|_{F}^{2}\right]}{n_{t}}-\left[\left(1-2^{\frac{-B_{k, c, c}}{n_{t}-1}}\right) \delta_{k, c}+\left(2^{\frac{-B_{k, c, c}}{n_{t}-1}}\right) \bar{\gamma}_{c}\right]\right) \\
& =\left(\bar{\gamma}_{c} n_{t} 2^{\frac{-B_{k, c, c}}{n_{t}-1}}+\left(1-2^{\frac{-B_{k, c, c}}{n_{t}-1}}\right) \xi_{c}-\left[\left(1-2^{\frac{-B_{k, c, c}}{n_{t}-1}}\right) \delta_{k, c}+\left(2^{\frac{-B_{k, c, c}}{n_{t}-1}}\right) \bar{\gamma}_{c}\right]\right),
\end{aligned}
$$

where, using Result 1 , the value of $\xi_{c}$ is

$$
\xi_{c}=\frac{\mathrm{D}_{c}^{(2)}}{n_{t}}
$$

Similarly, the interference at the $k^{\text {th }}$ user in the $c^{\text {th }}$ cell from the $j^{\text {th }}$ cell user, is given by

$$
\tilde{\psi}_{k, j}=\left(\bar{\gamma}_{j} n_{t} 2^{\frac{-B_{k, c, j}}{n_{t}-1}}+\left(1-2^{\frac{-B_{k, c, j}}{n_{t}-1}}\right) \xi_{j}-\left[\left(1-2^{\frac{-B_{k, c, j}}{n_{t}-1}}\right) \delta_{k, j}+\left(2^{\frac{-B_{k, c, j}}{n_{t}-1}}\right) \bar{\gamma}_{j}\right]\right) .
$$

The interference from any single interfering source at the $k^{\text {th }}$ user from the $c^{\text {th }}$ cell and $j^{\text {th }}$ cell are given by $\psi_{k, c}^{\prime}=\tilde{\psi}_{k, c} /\left(n_{t}-1\right)$ and $\psi_{k, j}^{\prime}=\tilde{\psi}_{k, j} /\left(n_{t}-1\right)$, respectively. Therefore, the expected interference in (7.36) can be written as

$$
I_{k, c}^{\prime}=\frac{P_{k, c, c}}{\bar{\gamma}_{c}}(K-1) \psi_{k, c}^{\prime}+\sum_{\substack{j=1 \\ j \neq c}}^{C} \frac{P_{k, c, j}}{\bar{\gamma}_{j}} K \psi_{k, j}^{\prime} .
$$

Expected SINR with RVQ: We can now express the expected SINR approximation in (7.25) for RVQ codebooks, by using (7.35) and (7.40), as

$$
\mathbb{E}\left[\widetilde{\operatorname{SINR}}_{k, c}\right] \approx \frac{\frac{P_{k, c, c}}{\bar{\gamma}_{c}}\left[\left(1-2^{\frac{-B_{k, c, c}}{n_{t}-1}}\right) \delta_{k, c}+\left(2^{\frac{-B_{k, c, c}}{n_{t}-1}}\right) \bar{\gamma}_{c}\right]}{1+\frac{P_{k, c, c}}{\bar{\gamma}_{c}}(K-1) \psi_{k, c}^{\prime}+\sum_{\substack{j=1 \\ j \neq c}}^{C} \frac{P_{k, c, j}}{\bar{\gamma}_{j}} K \psi_{k, j}^{\prime}} .
$$

Comparing (7.21) and (7.41), we note that when the number of feedback bits is large, the expected SINR approximation (7.41) approaches the expected SINR approximation with perfect $\mathrm{CDI}$ (7.21), such that $\mathbb{E}\left[\widetilde{\operatorname{SINR}}_{k, c}\right] \rightarrow \mathbb{E}\left[\mathrm{SINR}_{k, c}\right]$. The results derived in this section are used in developing an adaptive bit alloca- 
tion strategy for the coordinated RZF scheme. This is investigated in the next section.

\subsection{Adaptive Bit Allocation}

In limited feedback systems, the user assigns bits to the channels (serving and interfering) in each feedback period. Therefore, it is of interest to investigate adaptive bit allocation at the user for each feedback instance. The upper bound on the mean quantization errors associated with the error vectors in (7.41) can be leveraged to estimate how many bits should be allocated to the serving and interfering channels.

There are numerous studies $[51,128,136,142,143]$ on adaptive bit allocation for limited feedback systems. The majority of the schemes maximize/minimize a specific performance/distortion metric. While there are a few studies $[133,134]$ that consider RZF precoding with adaptive bit allocation for massive MISO systems, adaptive bit allocation with RZF precoding scheme is not well investigated for conventional (non-massive) MISO systems.

We propose an adaptive method to allocate the total number of bits at the user, $B=\sum_{i=1}^{C} B_{k, c, i}$, to quantize the serving channel and the out-of-cell interfering channels, by minimizing the mean interference at the user. It is important to note that minimizing the interference at each user in the system is in fact reducing the overall interference in the multicell network. The mean interference at the $k^{\text {th }}$ user in the $c^{\text {th }}$ cell in $(7.41)$, is given by

$$
\begin{aligned}
\mathrm{I}_{k, c}^{\prime} & =\frac{P_{k, c, c}}{\bar{\gamma}_{c}^{\prime}}(K-1) \psi_{k, c}^{\prime}+\sum_{\substack{j=1 \\
j \neq c}}^{C} \frac{P_{k, c, j}}{\bar{\gamma}_{j}^{\prime}} K \psi_{k, j}^{\prime} \\
& =\frac{P_{k, c, c}}{\bar{\gamma}_{c}^{\prime}} \frac{(K-1)}{n_{t}-1} \tilde{\psi}_{k, c}+\sum_{\substack{j=1 \\
j \neq c}}^{C} \frac{P_{k, c, j}}{\bar{\gamma}_{j}^{\prime}} \frac{K}{n_{t}-1} \tilde{\psi}_{k, j} .
\end{aligned}
$$

Substituting the values of $\tilde{\psi}_{k, c}$ and $\tilde{\psi}_{k, j}$ from (7.37) and (7.39) into (7.42) and rearranging, gives

$$
\begin{aligned}
I_{k, c}^{\prime}= & P_{k, c, c}(K-1) 2^{\frac{-B_{k, c, c}}{n_{t}-1}}\left(1-\Delta_{c}\right)+P_{k, c, c}(K-1) \Delta_{c} \\
& +\sum_{\substack{j=1 \\
j \neq c}}^{C} P_{k, c, j} K 2^{\frac{-B_{k, c, j}}{n_{t}-1}}\left(1-\Delta_{j}\right)+\sum_{\substack{j=1 \\
j \neq c}}^{C} P_{k, c, j} K \Delta_{j},
\end{aligned}
$$

where $\Delta_{c}=\left(\xi_{c}-\delta_{k, c}\right) /\left(\bar{\gamma}_{c}\left(n_{t}-1\right)\right)$ and $\Delta_{j}=\left(\xi_{j}-\delta_{k, j}\right) /\left(\bar{\gamma}_{j}\left(n_{t}-1\right)\right)$. We can 
write $(7.43)$ as

$$
\begin{aligned}
I_{k, c}^{\prime} & =\underbrace{P_{k, c, c}(K-1)\left(1-\Delta_{c}\right)}_{\bar{P}_{k, c, c}} 2^{\frac{-B_{k, c, c}}{n_{t}-1}}+\sum_{\substack{j=1 \\
j \neq c}}^{C} \underbrace{P_{k, c, j} K\left(1-\Delta_{j}\right)}_{\bar{P}_{k, c, j}} 2^{\frac{-B_{k, c, j}}{n_{t}-1}} \\
& +\underbrace{P_{k, c, c}(K-1) \Delta_{c}+\sum_{\substack{j=1 \\
j \neq c}}^{C} P_{k, c, j} K \Delta_{j}}_{P_{I}} \\
& =\bar{P}_{k, c, c} 2^{\frac{-B_{k, c, c}}{n_{t}-1}}+\sum_{\substack{j=1 \\
j \neq c}}^{C} \bar{P}_{k, c, j} 2^{\frac{-B_{k, c, j}}{n_{t}-1}}+P_{I} \\
& =\sum_{i=1}^{C} \bar{P}_{k, c, i} 2^{\frac{-B_{k, c, i}}{n_{t}-1}}+P_{I} .
\end{aligned}
$$

It is noted that the expected interference in (7.44) is composed of two terms, where the first term is the sum of the product of channel gains (with pathloss and shadowing) and codebook quantization errors. On the other hand, the second term in (7.44) is the sum of weighted channel gains independent of codebook quantization errors. Therefore, in order to solve for the number of bits that minimizes the mean interference at the $k^{\text {th }}$ user in the $c^{\text {th }}$ cell, we define an optimization problem, given by

$$
\begin{aligned}
& \min _{B_{k, c, 1}, \ldots, B_{k, c, C} \in\left\{0, \mathbb{R}^{+}\right\}} \sum_{i=1}^{C} \bar{P}_{k, c, i} 2^{\frac{-B_{k, c, i}}{n_{t}-1}} \\
& \text { s.t } \sum_{i=1}^{C} B_{k, c, i} \leq B
\end{aligned}
$$

where $\mathbb{R}^{+}$denotes the set of positive real numbers. This is a convex optimization problem as the objective function is logarithmically convex [128, 143]. Hence, we find the solution in a real space and discretize to the nearest point [128]. The Lagrangian function is

$$
L\left(B_{k, c, i}, \lambda\right)=\sum_{i=1}^{C} \bar{P}_{k, c, i} 2^{\frac{-B_{k, c, i}}{n_{t}-1}}+\lambda\left(\sum_{i=1}^{C} B_{k, c, i}-B\right),
$$

where $\lambda$ denotes the Lagrange multiplier. The first order optimality Karush- 
Kuhn-Tucker (KKT) conditions are given by

$$
\begin{aligned}
& \frac{\partial L\left(B_{k, c, i}, \lambda\right)}{\partial B_{k, c, i}}=-\frac{\ln (2) \bar{P}_{k, c, i}}{n_{t}-1} 2^{\frac{-B_{k, c, i}}{n_{t}-1}}+\lambda=0 \\
& \frac{\partial L\left(B_{k, c, i}, \lambda\right)}{\partial \lambda}=\sum_{i=1}^{C} B_{k, c, i}-B=0 .
\end{aligned}
$$

Solving (7.48) and (7.49), yields the number of bits required by the $k^{\text {th }}$ user in the $c^{\text {th }}$ cell to quantize the serving and out-of-cell interfering channels, such that the mean interference is minimized. This gives

$$
B_{k, c, c}^{*}=\min \left\{B, \Re\left(\left\lfloor\frac{B}{C}+\left(n_{t}-1\right) \log _{2}\left(\frac{\left(P_{k, c, c}(K-1)\left(1-\Delta_{c}\right)\right)^{(C-1) / C}}{\left(\prod_{j=1, j \neq c}^{C} P_{k, c, j} K\left(1-\Delta_{j}\right)\right)^{1 / C}}\right)\right\rfloor^{+}\right)\right\}
$$

where $\lfloor\cdot\rfloor^{+}=\max \{0, \cdot\}$. From $(7.50)$, we can conclude that the bit allocation is a function of serving and interfering channel powers at the user, the number of users, $K$, the number of cells, $C$, and the number of transmit antennas at each BS, $n_{t}$. The solution obtained in (7.50) requires $K>1$. Although the case when $C=2$ is straight forward, it is important to note that for $C>2$, all the interfering channels at the user are given an equal number of the remaining bits for quantization, after solving (7.50), such that $B_{k, c, j}^{*}=\left(B-B_{k, c, c}^{*}\right) /(C-1)$, $\forall j$, where $j \neq c$.

\subsection{Regularization Parameter}

The optimization of the regularization parameter is considered to be a difficult problem in multicell systems [144]. The regularization parameter is optimized in a single-cell scenario in [77], such that it maximizes SINR. In [145], for single-cell non-homogeneous MU systems, the regularization parameter is defined as

$$
\zeta_{c}=\frac{1}{K} \sum_{k=1}^{K} 1 / P_{k, c, c}
$$

As the multicell system considered in this paper consists of serving and out-ofcell interfering channels with different link gains, we extend the regularization parameter in [145], such that

$$
\tilde{\zeta}_{c}=\frac{1}{C K} \sum_{i=1}^{C} \sum_{k=1}^{K} 1 / P_{k, c, i}
$$


In this study, we also consider an optimal regularization parameter, denoted by $\zeta_{c}^{o p t}$, that maximizes the spectral efficiency of the cell [133]. This optimal regularization parameter is given by

$$
\zeta_{c}^{\mathrm{opt}}=\arg \max _{\zeta_{c}^{\text {opt }}>0} \sum_{k=1}^{K} \log _{2}\left(1+\operatorname{SINR}_{k, c}\right) .
$$

Finding the optimal regularization parameter, $\zeta_{c}^{\text {opt }}$, that maximizes the spectral efficiency (7.53) is analytically difficult, therefore we numerically compute $\zeta_{c}^{\text {opt }}$ to evaluate the performance of the coordinated RZF scheme in the next section.

\subsection{Numerical Results and Discussion}

We now present simulation results for a multicell MU MISO system with coordinated RZF precoding. We divide this section into three parts, beginning by explaining the concept of the coordination area in the cell, followed by comparing the expected SINR approximation for perfect CDI and RVQ codebook CDI derived in (7.21) and (7.41), respectively, with the corresponding simulated average SINRs. We present average cell-edge spectral efficiency for perfect CDI and limited feedback based RVQ CDI under different transmission scenarios. Next, we present the performance of the coordinated RZF scheme with the adaptive bit allocation method and compare it with the coordinated ZF scheme [128], for different numbers of coordinating cells. Finally, we remove the coordination area restriction and present the average cell spectral efficiency comparison between the coordinated RZF scheme and the coordinated ZF scheme [128]. We assume i.i.d. Rayleigh block fading. The radius of the single cell is set to $R_{c}=500 \mathrm{~m}$. The standard deviation of the shadowing is $8 \mathrm{~dB}$ and the path loss exponent is $a=3.8$ [146].

\subsubsection{Coordination Area}

We define the coordination area as an area in the cell where users may experience high interfering power from the adjacent cells. The coordination area is based on the user's distance. As there is no such formal definition of the coordination area, we follow the arbitrary definition given in [128], i.e., coordination is needed when the user lies in the region $325 \mathrm{~m} \leq d \leq 500 \mathrm{~m}$. The two- and three-cells coordination areas are illustrated in Fig. 7.2(a) and Fig. 7.2(b), respectively. The users are dropped in the coordination area uniformly and such users are called cell-edge users. 


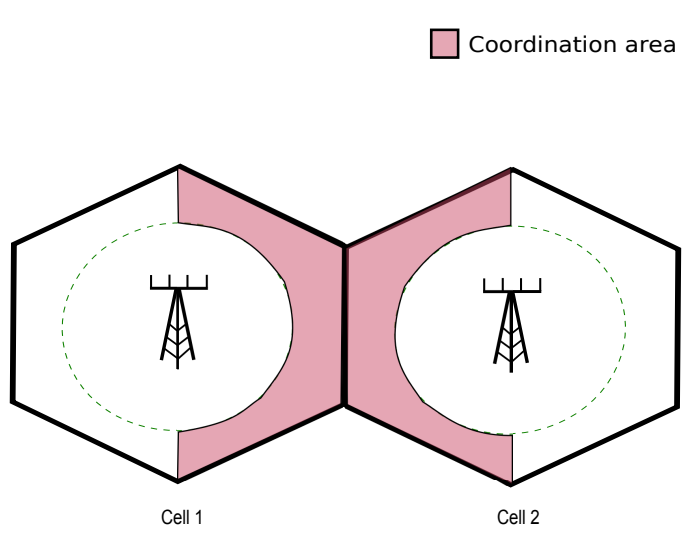

(a) Coordination area in two-cell simulations.

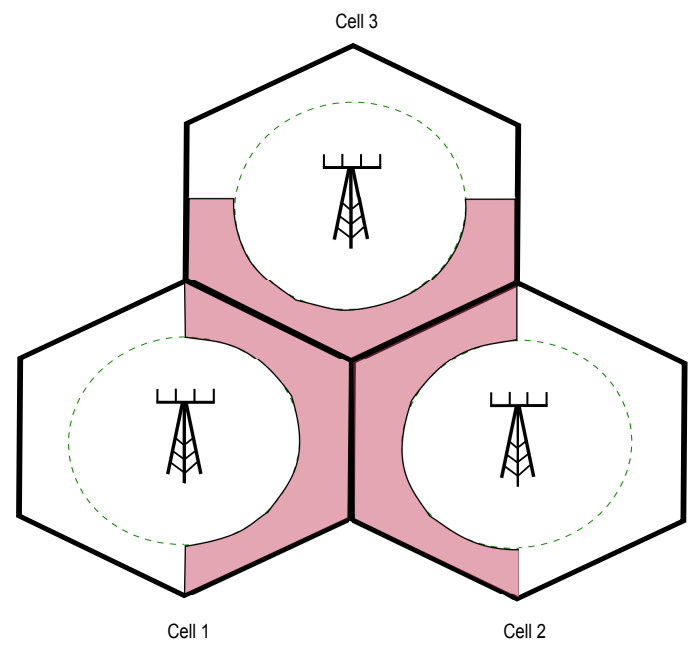

(b) Coordination area in three-cell simulations.

Figure 7.2: Illustration of two-cell and three-cell coordination areas in the simulations.

\subsubsection{SINR and Spectral Efficiency}

In this section, we present SINR and spectral efficiency results for the proposed coordinated RZF scheme. We plot these results against the average received celledge SNR, $\rho_{0}$, obtained by substituting the link distance equal to $R_{c}$ in (7.2) given by $\rho_{0}=\mathbb{E}\left[10 \log _{10}\left(P_{0} 10^{\left(\eta \sigma_{S F} / 10\right)} / N_{0}\right)\right]$, where the noise power $N_{0}=1$ and $\eta \sim \mathcal{N}(0,1)$. It is important to mention that increasing $\rho_{0}$ also increases the SNR at the users. The increase in $\rho_{0}$ also increases interference to the users (in the same or neighboring cells). Figure 7.3 shows the average SINR performance of the coordinated RZF scheme with perfect CDI and limited feedback based RVQ CDI. The approximate expected SINRs derived in (7.21) and (7.41) are plotted (in the linear scale) in Fig. 7.3 for perfect CDI and RVQ CDI with various numbers of feedback bits. It is observed that the approximations of the expected SINR with perfect CDI matches the average simulated spectral efficiency, however the expected SINR approximation (7.41) with an RVQ codebook shows a small deviation relative to the simulated average SINR at $\rho_{0}$ values. The average cell-edge spectral efficiency for $C=2$ and $C=3$ cells with $K=2$ cell-edge users is shown in Fig. 7.4 , where each BS has $n_{t}=6$ antennas. Denoting the cell-edge spectral efficiency by $C_{\text {edge }}$, the average cell-edge spectral efficiency simulations are performed using

$$
\mathbb{E}\left[C_{\text {edge }}\right]=\mathbb{E}\left[\sum_{k=1}^{K} \log _{2}\left(1+\operatorname{SINR}_{k, c}\right)\right],
$$




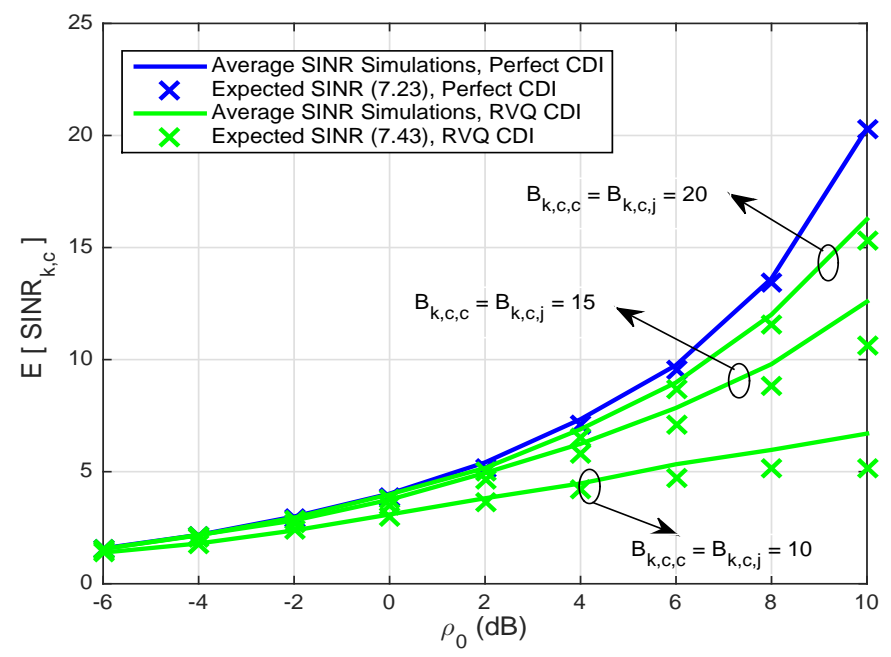

Figure 7.3: The average SINR for the $k^{\text {th }}$ user in the $c^{\text {th }}$ cell, with $C=2$ cells and $K=2$ users where each BS has $n_{t}=4$ antennas with $B_{k, c, c}=B_{k, c, j}=20$, 15 and 10.

where $\operatorname{SINR}_{k, c}$ is given in (7.5) and the users are located in the cell-edge area. Therefore we refer to (7.54) as the average cell-edge spectral efficiency of the cell. This is plotted against different $\rho_{0}$ values in Fig. 7.4. The single cell MU system gives superior average cell-edge spectral efficiency compared to the other scenarios, due to the absence of ICI. However, when ICI is present, the average cell-edge spectral efficiency with the non-coordinated RZF precoding scheme suffers high losses and the performance gap increases compared to the single-cell case, as $\rho_{0}$ increases. For the coordinated RZF scheme, we consider two cases: 1) only 2 out of 3 cells coordinate and 2) all 3 cells coordinate. In Fig. 7.4, we consider two regularization parameters for the coordinated RZF case 2: $\zeta_{c}$ and $\zeta_{c}^{\mathrm{opt}}$.

The coordinated RZF case 2 with $\zeta_{c}^{\text {opt }}$ achieves better average cell-edge spectral efficiency compared to the coordinated RZF case 1 (with $\zeta_{c}$ ) and the noncoordinated RZF scheme. The coordinated RZF schemes with both cases 1 and 2 outperform the coordinated ZF [128] scheme. However, for $\rho_{0}<2 \mathrm{~dB}$ and $\rho_{0}<3 \mathrm{~dB}$ with case 1 and case 2, respectively, it is noted in Fig. 7.4(a) that the coordinated RZF schemes with $\zeta_{c}$ are not effective and the non-coordinated RZF scheme is more effective, as the former leverages the benefit of fewer channels being orthogonalized while precoding, thus leading to a stronger signal power. The same reasoning is applicable to the proposed coordinated RZF case 1 which yields better performance than the proposed coordinated RZF case 2 , for $\rho_{0}<8$ $\mathrm{dB}$, with the same number of antennas at the BS (i.e., $\left.n_{t}=6\right)$. In Fig. 7.4(b), we plot the same cases as Fig. 7.4(a) with $n_{t}=8$ antennas at the BS. We observe that for $\rho_{0}>-4 \mathrm{~dB}$, the proposed coordinated RZF schemes using $\zeta_{k}$ outperforms the non-coordinated RZF scheme. Again, the performance of the 


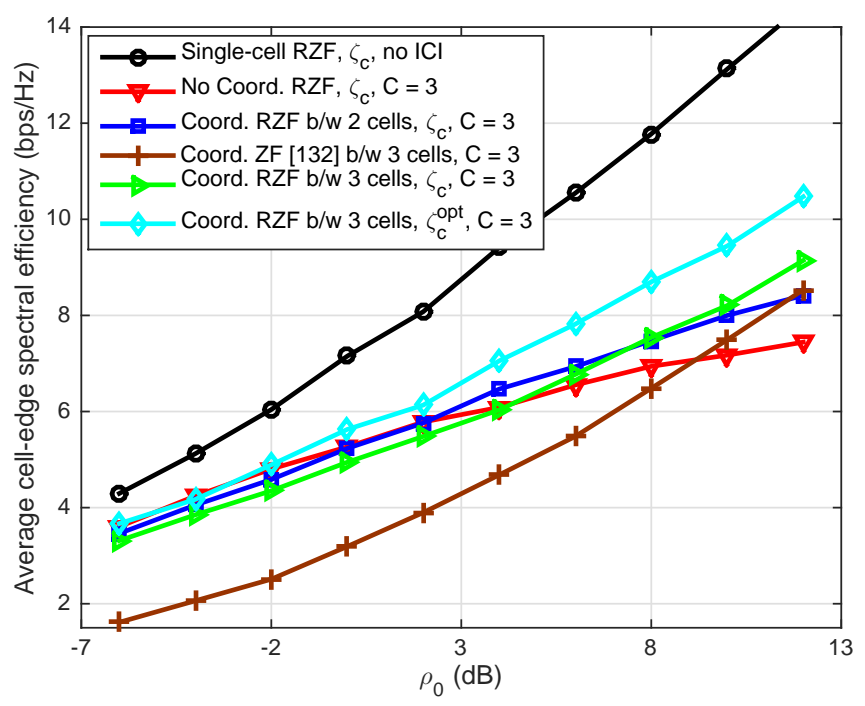

(a) $n_{t}=6$

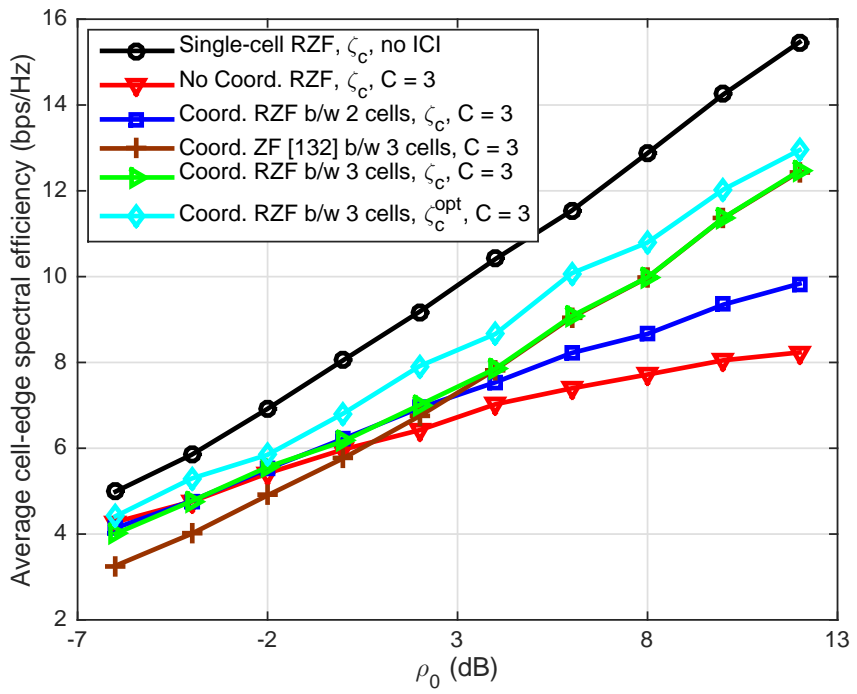

(b) $n_{t}=8$

Figure 7.4: The average cell-edge spectral efficiency with $C=3$ cells for $K=2$ users.

proposed RZF case 2 with $\zeta_{c}^{\text {opt }}$ is higher than all the other scenarios. Therefore, by comparing Fig. 7.4(a) and Fig. 7.4(b), we note that the coordination gain can be obtained over the non-coordinated RZF method, for a range of $\rho_{0}$ values by either increasing $n_{t}$, such that $n_{t}>C K$, or by using an effective regularization parameter (here, $\zeta_{c}^{\text {opt }}$ ). 


\subsubsection{Performance of Adaptive Bit Allocation Scheme}

We evaluate the average cell-edge spectral efficiency of the coordinated RZF precoding scheme with the adaptive bit allocation scheme discussed in Section 7.4, when the numbers of coordinating cells are $C=2$ and 3. From this point onwards, we use the regularization parameter $\tilde{\zeta}_{c}$ given in (7.52).

\section{Coordination with 2 cells}

The average cell-edge spectral efficiency for the proposed adaptive bit allocation scheme is shown in Fig. 7.5 with $B=8, n_{t}=4$ and $K=2$. It is compared with

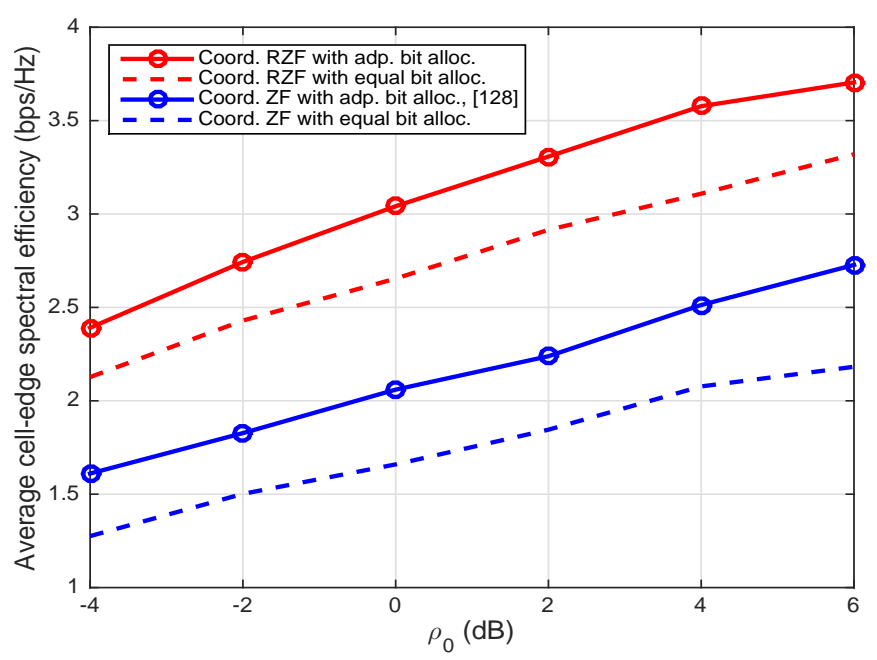

Figure 7.5: The average cell-edge spectral efficiency with $C=2$ cells and $K=2$ users where each BS has $n_{t}=4$ antennas with $B=8\left(\sigma_{S F}=8 \mathrm{~dB}\right)$.

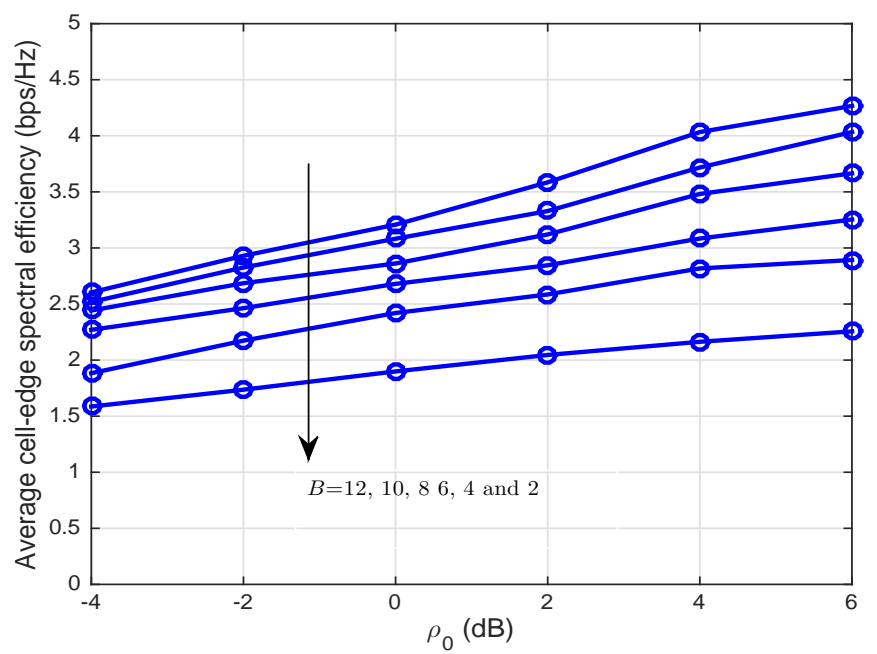

Figure 7.6: The average cell-edge spectral efficiency with $C=2$ cells and $K=2$ users where each BS has $n_{t}=4$ antennas with $\sigma_{S F}=8 \mathrm{~dB}$. 


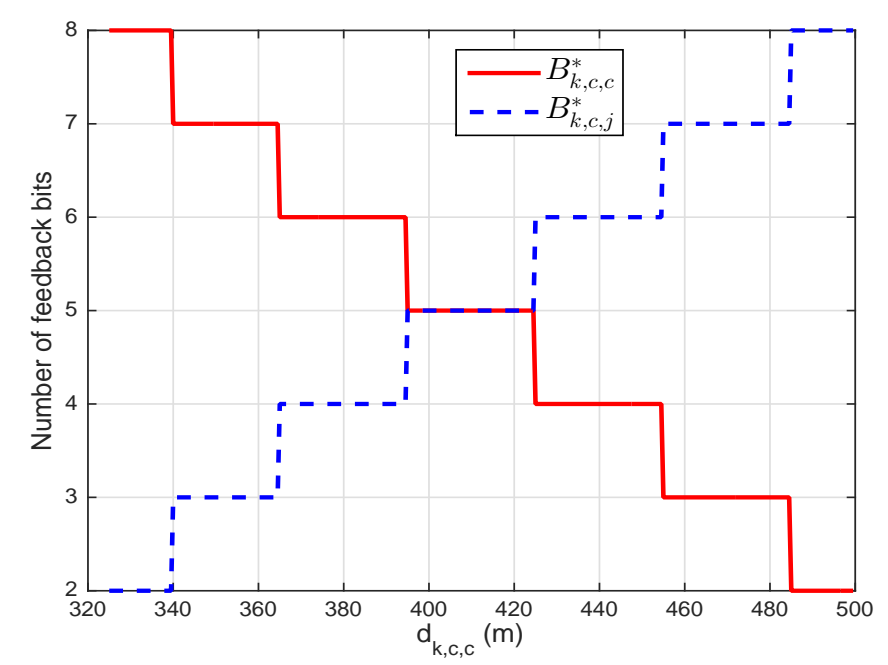

Figure 7.7: The average proposed adaptive bit allocation for the $k^{\text {th }}$ user in the $c^{\text {th }}$ with $\rho_{0}=5 \mathrm{~dB}, C=2$ cells and $K=2$ users where each BS has $n_{t}=4$ antennas with $B=10$ (no shadowing).

the coordinated ZF adaptive bit allocation scheme [128]. The scenario used in the simulation is the $C=2$ case depicted in Fig. 7.2(a). It is seen that the coordinated RZF scheme improves the average cell-edge spectral efficiency compared to the coordinated ZF adaptive bit allocation scheme [128]. Even equal bit partitioning (i.e., 4 bits) to each serving and out-of-cell interfering channel, results in better average cell-edge spectral efficiency than the coordinated ZF adaptive bit allocation scheme [128]. The effect of quantization errors on the average cell-edge spectral efficiency is shown in Fig. 7.6 for the coordinated RZF scheme with $n_{t}=4, K=2$ and various $B$ values. As the total number of bits at the user increases, quantization errors decrease which results in higher spectral efficiency performance. The average number of bits allocated to the serving and interfering channels with the adaptive bit allocation scheme is shown in Fig. 7.7 for coordinated RZF with $B=10$ bits and $\rho_{0}=5 \mathrm{~dB}$. It shows the average bit allocation trend with respect to different distance values. The adaptive bit allocation scheme divides bits equally among the serving and interfering channels in the region $395 \mathrm{~m} \leq d_{k, c, c} \leq 425 \mathrm{~m}$. As the distance increases, the number of bits allocated to the serving channel decreases.

\section{Coordination with 3 cells}

For $C=3$ cells, we use the scenario shown in Fig. 7.2(b). The average cell-edge spectral efficiency is shown in Fig. 7.8 for $C=3$ cells using the coordinated RZF with adaptive bit allocation strategy, where $B=9, n_{t}=6$ and $K=2$. The trend is similar to Fig. 7.5, and the bit allocation strategy (7.50) yields better average cell-edge spectral efficiency compared to the coordinated ZF with 


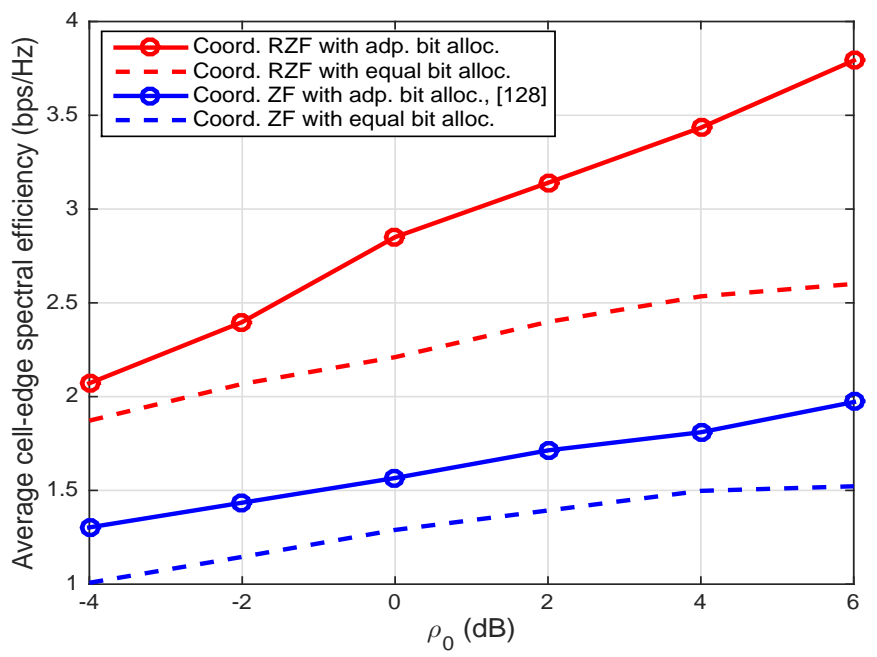

Figure 7.8: The average cell-edge spectral efficiency with $C=3$ cells and $K=2$ users where each BS has $n_{t}=6$ antennas with $B=9\left(\sigma_{S F}=8 \mathrm{~dB}\right)$.

adaptive bit allocation method.

\section{Full cell area coordination}

Here we assume that there is no restriction on the coordination area, i.e., users always coordinate regardless of their position in the cell. Figure 7.9 shows average cell spectral efficiency for the coordinated RZF and the coordinated ZF precoding [128] strategies at $\rho_{0}=5 \mathrm{~dB}$ (no shadowing). The performance is shown for both adaptive bit allocation and equal bit allocation, with and without shadowing. In all the four cases plotted, the average cell spectral efficiencies

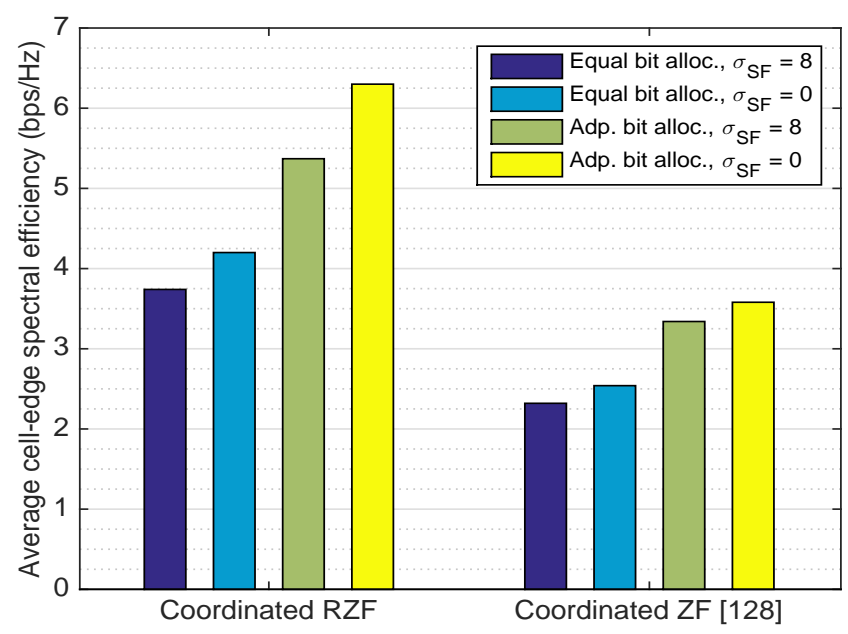

Figure 7.9: The average cell spectral efficiency with $C=3$ coordinating cells having $K=2$ users where each BS has $n_{t}=6$ antennas with $B=9$. 
with the coordinated RZF scheme are higher than the coordinated ZF scheme. Hence, the effectiveness of the coordinated RZF scheme is not only limited to the cell-edge, but it also yields acceptable performance across the entire cell area.

\subsection{Summary}

In this chapter we have discussed the coordinated RZF precoding technique for multicell MU MISO systems. We derived expected SINR approximations for both perfect CDI and limited feedback RVQ based CDI. Via simulations, we show that the coordinated RZF precoding outperforms the non-coordinated RZF at high $\rho_{0}$ values with a suitable choice of the regularization parameter. We presented an adaptive feedback bit allocation scheme with limited feedback RVQ CDI that minimizes the mean interference at the user. The adaptive bit allocation with coordinated RZF was shown to yield higher average celledge spectral efficiency performance than the equal bit allocation and existing coordinated ZF based adaptive bit allocation schemes. 


\section{8 \\ Conclusions and Future Work}

In this chapter, we present summary and conclusion of the research reported in this thesis. We also provide directions for future research in this area.

\subsection{Summary}

As discussed in previous chapters, the quality of CSI at the transmitter has a very large impact on the performance of a wireless communication system. In this thesis, we have focused on limited feedback techniques for multi-antenna systems and codebook adaptation. The aim of the study was mainly to design effective codebooks that quantizes CDI with a minimum quantization error at the receiver for temporally/spatially correlated channels. For this purpose, we exploited the temporal and/or spatial correlation present in the channel and used this information to design various differential codebook structures. With massive antennas at the transmitter, we developed TCQ based limited feedback schemes for temporally and spatially correlated channels that rely on TCQ encoding at the receiver to quantize CDI. In massive MISO systems, the quantization with TCQ has lower search complexity than conventional codebook quantization schemes. For multicell system we have considered coordinated RZF precoding with adaptive bit allocation in Rayleigh i.i.d. channels. We present summary of each chapter of the thesis below.

\section{Capacity Loss Analysis in SU MIMO}

We first investigated the capacity loss in SU MIMO systems due to the standard codebooks: Grassmannian and 3GPP LTE. As discussed in Chapter 4, we considered right singular vectors as precoders and limited the study to single- 
and two-streams transmissions. On the receiver side, three linear receivers were considered: ZF, MMSE and SVD. We also derived capacity loss expressions as a function of codebook quantization errors.

\section{Differential Codebooks in MU MISO}

After investigating the capacity loss in SU MIMO systems, we shifted the focus towards MU MISO systems, where unlike SU MIMO, there is no multi-stream interference to suppress at the receiver; instead, there is MU interference. The transmitter cannot practically obtain perfect CSI and precoders are computed with quantized CSI and thus the system exhibits performance loss due to codebook quantization errors.

In temporally correlated channels, the channel entries change slowly over time. Using this property of temporally correlated channels, we developed differential codebooks in Chapter 5, where single spherical-cap and double-cap designs are investigated.

\section{Spherical-cap Differential Codebook}

In spherical-cap differential codebooks, the fixed Grassmannian codewords are scaled to lie closer to each other packed in a cap with the fixed radius. The radius of the cap is the distance between the center of the cap and the codeword located furthest from the center. The spherical-cap is rotated after each feedback interval to follow the changing channel. Two adaptive scaling techniques using a spherical-cap are developed: Adaptive Scaling Technique I and II.

\section{Double-cap Differential Codebook}

In addition to single spherical-cap design, we have also designed a double-cap differential codebook that overcomes the disadvantages of both Adaptive Scaling Techniques I and II. The double-cap differential codebook does not require any additional information or knowledge at the transmitter and also works well for longer transmission periods. The double-cap design consists of a sphericalcap as inner cap and a polar-cap as outer cap (with codewords on the cap circumference). This two-cap design allows us to perform systematic scaling up or down of the double-cap differential codebook to follow the slowly varying channel. Depending on the system requirements, either spherical-cap or doublecap differential codebook designs can be employed. We also introduced a SVD based rotation method to direct the codewords towards the desired position. 


\section{MU Massive MISO with TCQ}

In Chapter 6, we investigated a non-codebook based limited feedback technique in MU massive MISO systems for temporally and spatially correlated channels. Conventional codebook approaches are not feasible for massive MISO systems because as the number of transmit antennas increases, the required number of codewords in the codebook also increase. This increase makes a search for an appropriate codeword in the codebook computationally impractical. Using this as the motivation, we adapt the TCQ approach to quantize the channel at the user. For temporally correlated channels, we have developed a differential TCQ method that exploits temporal correlation in the channel and transforms (rotates and scales) the source constellation after each feedback interval. The scaling parameter is a function of temporal correlation and the number of transmit antennas. We have also presented a TCQ based limited feedback scheme for spatially correlated channels.

\section{Multicell MU MISO with Coordination}

Coordinated RZF scheme for MU MISO systems with codebook-based limited feedback is discussed in chapter 7 for Rayleigh i.i.d. channels. We have derived expected SINR approximations for the coordinated RZF scheme with perfect CDI and RVQ based quantized CDI. We have also presented an adaptive bit allocation scheme that minimizes interference at the user.

\subsection{Conclusions}

In this thesis, we used limited feedback techniques to equip the transmitter with CSI and investigated their performance in several wireless communication networks: SU/MU single-cell and MU multicell. In Chapter 4, we demonstrated that the MIMO capacity loss due to codebooks is closely related to minimum squared chordal distance between the right singular vectors of the channel and the codebook entries. The analysis provides meaningful insights on the MIMO capacity loss in the presence of codebook quantization errors. Interestingly, we observed that in single-stream MIMO systems, the performance of spatially correlated channels is better than the performance with i.i.d. Rayleigh fading channels. This is due to the fact that the dominant eigenmode of a spatially correlated channel is very strong compared to other eigenmodes.

Differential codebooks with adaptive scaling techniques developed in Chapter 5 have shown to effectively track temporally correlated channels. Three adaptive scaling techniques are designed where Adaptive Scaling Technique II requires additional channel statistics. Adaptive scaling Technique I is reset to the base codebook after a few feedback intervals. The results confirm that 
there is a trade-off between the performance of adaptive scaling techniques and additional feedback of channel statistics. In Chapter 6, for massive MISO systems, TCQ based limited feedback schemes are developed with temporally and spatially correlated channels. These schemes quantize large dimensional channel vectors with smaller quantization errors than the existing NTCQ schemes. Also, TCQ based limited feedback schemes reduce the search complexity at the receiver compared to conventional codebook-based limited feedback schemes. For temporally correlated channels, transformation of the source constellation according to channel statistics yield significant performance gains.

The RZF based coordinated precoding for MU MISO systems discussed in Chapter 7 has shown to provide better average cell-edge spectral efficiency than the ZF based coordination scheme, at low operating SNRs. The SINR approximations derived for perfect CDI and RVQ CDI are shown to be in close agreement with numerical simulations. The expected interference power is further utilized to adaptively allocate feedback bits to serving and interfering channels. This has shown to outperform equal feedback bit partitioning.

In the following section, we analyze the limitations of the thesis and discuss future work prospects.

\subsection{Limitations and Future Work}

\subsubsection{Limitations}

Although the thesis has reached its aims, there are some inevitable limitations. These limitations are briefly discussed below:

- Throughout the thesis, we assume that feedback links have no delays. In practice, the feedback delay exists due to factors such as signal processing and propagation [147]. The negative effects of delayed feedback on the performance of MIMO systems are studied in [148-151]. The delayed feedback results in a weak signal power and also introduces inter-stream interference [147]. To mitigate the effects of the delayed feedback, channel prediction based techniques are investigated in $[152,153]$.

- For MIMO-OFDM systems in Chapter 4, we do not investigate the frequency correlation between the subcarriers and select a separate codebook entry for each subcarrier. This increases the complexity at the receiver and also increases the feedback overhead, when the number of subcarriers is large. To reduce the feedback overhead, interpolation based techniques are presented in $[154,155]$ for MIMO-OFDM systems. In [154], the correlation between the beamforming vectors of subcarriers is explored to reduce the feedback overhead. 
- Differential codebooks with adaptive scaling techniques (discussed in Chapter 5) have shown to perform well under highly temporally correlated channels. However, the performance of the differential codebooks degrades when the user velocity increases. Specifically, those schemes which do not reset to the base codebook have shown to suffer the most under these scenarios. It will be useful to modify these adaptive scaling schemes such that they can successfully track both slowly and moderately varying channels.

- Majority of the results presented in Chapter 5 assume perfect channel estimation at the user, however it is seen that estimation errors have a detrimental effect on the performance of differential codebooks. In Section 5.9 .3 , it is shown that even the small estimation error can degrade the performance of the differential codebook, especially for the double-cap structure. In [150], estimation errors have shown to increase bit error rate (BER) and outage probability of the MIMO-SVD system. The negative effects of estimation errors can be overcome by increasing the size of the codebook.

\subsubsection{Future Work}

Limited feedback techniques are supported in current networks such as 3GPP LTE release 8. The use of limited feedback techniques may also become part of future $5 \mathrm{G}$ networks. $5 \mathrm{G}$ technologies such as millimeter wave (mmWave) and massive MIMO are being widely investigated with limited feedback schemes. We present the future research in the following categories:

\section{Extension to MIMO Systems}

We have developed differential codebooks for MISO systems in Chapter 5 and assumed that the receiver is equipped with a single antenna. However, in MIMO systems with multiple antennas at both the transmitter and the receiver, the codebook (unlike that for MISO) consists of matrices with orthonormal columns [74]. One possible approach is to use a set of orthogonal spherical-caps on the Grassmannian manifold. A similar solution is proposed in [45] for spatially correlated MIMO channels. For temporally correlated MIMO channels, a rotation based differential codebook technique is investigated in [114]. The drawback of this scheme is that error propagates over time as precoding matrices depend on previous selected precoding matrices. Further studies are needed to address this issue.

In Chapter 6, we presented TCQ based limited feedback schemes for massive MISO channels. The extension to massive MIMO channels is not straightforward. If each column of the MIMO channel matrix is quantized separately using 
the TCQ approach, then the feedback overhead increases $n_{r}$ times compared to the MISO channel. It will be interesting to investigate the massive MIMO channel by modifying the TCQ design, in a manner that the number of feedback bits does not increase significantly for massive MIMO systems.

Unlike massive MISO channels, massive MIMO channels can exhibit spatial correlation at both the transmitter and receiver. This will increase the complexity of system analysis and will further impact the performance of massive MIMO systems with limited feedback.

\section{Heterogeneous Networks}

Heterogeneous networks (HetNets) employ a number of small cells in the network to achieve high spectral efficiency [156]. Recently, researchers have investigated the performance of multi-tier HetNet with limited feedback schemes $[52,157]$. In [157], the effect of feedback delay and quantization error on the achievable macrocell sum-rate is investigated with transmit beamforming and uncoordinated cross-tier interference. A low power vector perturbation (VP) is designed for small cells in [158] which yields 300\% improvement in power efficiency levels compared to conventional VP. For future work, it is worth exploring coordinated non-linear beamforming strategies which increase the power efficiency and also improve the cell spectral efficiency with limited feedback schemes. Coordination schemes in HetNet are studied in [159] where various levels of coordination between two-tier networks are presented.

In $5 \mathrm{G}$ systems, network coordination is considered to be an important component for improving spectral efficiency and reducing ICI. However, the use of massive MIMO and densely deployed small cells will increase the need of coordination and precise CSI [160]. This issue needs to be addressed by optimizing the coordination and improving the codebook design having a low codeword search complexity. Therefore, the coupling of the network coordination with $5 \mathrm{G}$ systems requires more robust and practical schemes.

\section{MmWave Communication and Hybrid Precoding}

MmWave communication recently gained attention for the development of $5 \mathrm{G}$ communication networks. MmWave technology can yield multi-Gigabit throughput by operating in $\mathrm{GHz}$ band. The effects of higher path loss at mmWave frequencies are compensated by using massive MIMO which provides array gain and also reduces the power consumption [161-163]. The conventional digital beamforming techniques consume high power in massive arrays. Therefore, to minimize the power consumption, directional beamforming should be employed by mean of analog beamforming techniques. For this purpose analog beamforming schemes are investigated in [164-166] for SU systems and hybrid (both 
analog and digital) precoding for SU systems in [167] with partial knowledge of the mmWave channel. In [168], a hybrid precoding scheme is proposed for MU mmWave channels with limited feedback. The performances of several mmWave precoding schemes are studied in [169] for various codebook based RF beam selection strategies at the transmitter and receiver.

Recently, hybrid beamforming schemes have been developed for SU MIMO systems in [170] that jointly optimize analog and digital beamforming matrices to maximize the spectral efficiency of the system. These hybrid beamforming schemes outperform the beam steering method [171], but have a high complexity. Here it is assumed that each antenna is connected with a unique RF chain, however, in massive multi-antenna systems, assigning each transmit antenna with a unique RF chain is very costly. Also, power consumption is high for RF hardware. To address these issues a hybrid beamforming has been proposed for MU MISO systems in [172], where instead of each transmit antenna each user is assigned a single RF chain, thus reducing the number of RF chains at the transmitter for systems where the number of transmit antennas is large compared to the number of users. It is noted in [172] that the spectral efficiency with hybrid beamforming is lower than the conventional digital beamforming methods, but this loss can be compensated by increasing the number of transmit antennas by $27 \%$. However, the study presented in [172] considers uncorrelated Rayleigh channels and not mmWave channels. Therefore, the impact of reducing the $\mathrm{RF}$ chains on the performance of massive mmWave arrays requires further investigation with limited feedback techniques.

In order to understand mmWave channels, 3D mmWave channel models are proposed in [173] and [174] for $28 \mathrm{GHz}$ and $73 \mathrm{GHz}$, respectively. Due to reduced scattering, the channel becomes more sparse, and so conventional codebooks may not perform well in mmWave channels. Future studies are needed to address this issue by developing effective limited feedback techniques for mmWave channels. 
Appendices 



\section{A}

\section{Proof of Result 1}

In this appendix, we prove that if the entries of an $n_{t} \times n_{t}$ matrix $\mathbf{H}$ are i.i.d. $\mathcal{C N}(0,1)$, then the expected value of $\sum_{n=1}^{n_{t}} \frac{\left(\lambda_{n}\right)^{t}}{\left(\lambda_{n}+\zeta_{c}\right)^{2}}$, where $\lambda_{n}$ is the $n^{\text {th }}$ eigenvalue of $\mathbf{H H}^{H}$ (uncorrelated central Wishart matrix), with respect to $\lambda_{n} \forall n$, is given by

$$
\begin{aligned}
\mathrm{D}_{c}^{(t)}= & \mathbb{E}\left[\sum_{n=1}^{n_{t}} \frac{\left(\lambda_{n}\right)^{t}}{\left(\lambda_{n}+\zeta_{c}\right)^{2}}\right] \\
= & \sum_{i=1}^{n_{t}} \sum_{j=0}^{i-1} \sum_{l=0}^{i-1}(-1)^{j+l}\left(\begin{array}{c}
i-1 \\
i-1-j
\end{array}\right)\left(\begin{array}{c}
i-1 \\
i-1-l
\end{array}\right) \frac{1}{j ! l !} \sum_{s=0}^{t+j+l}\left(\begin{array}{c}
t+j+l \\
s
\end{array}\right) \\
& \left(-\zeta_{c}\right)^{t+j+l-s} \mathrm{e}^{\zeta_{c}} \int_{\zeta_{c}}^{\infty} v^{s-2} \mathrm{e}^{-v} d v .
\end{aligned}
$$

Let, $\mathrm{D}_{c}^{(t)}$ be defined by

$$
\begin{aligned}
\mathrm{D}_{c}^{(t)} & =\mathbb{E}\left[\sum_{i=1}^{n_{t}} \frac{\left(\lambda_{n}\right)^{t}}{\left(\lambda_{n}+\zeta_{c}\right)^{2}}\right] \\
& =n_{t} \mathbb{E}\left[\frac{1}{n_{t}} \sum_{i=1}^{n_{t}} \frac{\left(\lambda_{n}\right)^{t}}{\left(\lambda_{n}+\zeta_{c}\right)^{2}}\right]
\end{aligned}
$$

Now any expectation of the form (A.2) can be written as

$$
\mathrm{D}_{c}^{(t)}=n_{t}\left[\int_{0}^{\infty} \frac{\left(\lambda_{1}\right)^{t}}{\left(\lambda_{1}+\zeta_{c}\right)^{2}} f_{\lambda_{1}}\left(\lambda_{1}\right) d \lambda_{1}+\cdots+\int_{0}^{\infty} \frac{\left(\lambda_{n_{t}}\right)^{t}}{\left(\lambda_{n_{t}}+\zeta_{c}\right)^{2}} f_{\lambda_{n_{t}}}\left(\lambda_{n_{t}}\right) d \lambda_{n_{t}}\right]
$$

where $f_{\lambda_{1}}, \ldots, f_{\lambda_{n_{t}}}$ are the PDFs of $\lambda_{1}, \ldots, \lambda_{n_{t}}$, respectively. We can also write 
(A.3) in terms of an arbitrary eigenvalue as

$$
\begin{aligned}
\mathrm{D}_{c}^{(t)} & =n_{t}\left[\int_{0}^{\infty} \frac{1}{n_{t}} \frac{(\lambda)^{t}}{\left(\lambda+\zeta_{c}\right)^{2}} f_{\lambda_{1}}(\lambda) d \lambda+\cdots+\int_{0}^{\infty} \frac{1}{n_{t}} \frac{(\lambda)^{t}}{\left(\lambda+\zeta_{c}\right)^{2}} f_{\lambda_{n_{t}}}(\lambda) d \lambda\right] \\
& =n_{t}\left[\int_{0}^{\infty} \frac{1}{n_{t}} \frac{(\lambda)^{t}}{\left(\lambda+\zeta_{c}\right)^{2}}\left(f_{\lambda_{1}}(\lambda)+\ldots+f_{\lambda_{n_{t}}}(\lambda)\right) d \lambda\right] \\
& =n_{t}\left[\int_{0}^{\infty} \frac{(\lambda)^{t}}{\left(\lambda+\zeta_{c}\right)^{2}} f_{0}(\lambda) d \lambda\right]
\end{aligned}
$$

where $f_{0}(\lambda)$ is the PDF of an arbitrary eigenvalue and is written as (for the case $\left.C K=n_{t}\right)[3,175]$

$$
f_{0}(\lambda)=\frac{1}{n_{t}} \sum_{i=1}^{n_{t}} \mathrm{e}^{-\lambda} \mathrm{L}_{i-1}(\lambda)^{2}
$$

where $\mathrm{L}_{i-1}(\cdot)$ is a Laguerre polynomial which can be also written as

$$
\mathrm{L}_{i-1}(\lambda)=\sum_{j=0}^{i-1}(-1)^{j}\left(\begin{array}{c}
i-1 \\
i-1-j
\end{array}\right) \frac{\lambda^{j}}{j !}
$$

Substituting (A.6) in (A.5), the PDF of an arbitrary eigenvalue becomes

$$
f_{0}(\lambda)=\frac{1}{n_{t}} \sum_{i=1}^{n_{t}} \mathrm{e}^{-\lambda}\left(\sum_{j=0}^{i-1}(-1)^{j}\left(\begin{array}{c}
i-1 \\
i-1-j
\end{array}\right) \frac{\lambda^{j}}{j !}\right)^{2} .
$$

Therefore, now (A.4) can be written as

$$
\begin{aligned}
\mathrm{D}_{c}^{(t)} & =\int_{0}^{\infty} \frac{(\lambda)^{t}}{\left(\lambda+\zeta_{c}\right)^{2}} \sum_{i=1}^{n_{t}} \mathrm{e}^{-\lambda}\left(\sum_{j=0}^{i-1}(-1)^{j}\left(\begin{array}{c}
i-1 \\
i-1-j
\end{array}\right) \frac{\lambda^{j}}{j !}\right)^{2} d \lambda \\
& =\sum_{i=1}^{n_{t}} \sum_{j=0}^{i-1} \sum_{l=0}^{i-1}(-1)^{j+l}\left(\begin{array}{c}
i-1 \\
i-1-j
\end{array}\right)\left(\begin{array}{c}
i-1 \\
i-1-l
\end{array}\right) \frac{1}{j ! l !} \int_{0}^{\infty} \frac{\lambda^{t+j+l} \mathrm{e}^{-\lambda}}{\left(\lambda+\zeta_{c}\right)^{2}} d \lambda
\end{aligned}
$$

Substituting $\lambda=v-\zeta_{c}$, the integral in (A.8) becomes

$$
\int_{0}^{\infty} \frac{\lambda^{t+j+l} \mathrm{e}^{-\lambda}}{\left(\lambda+\zeta_{c}\right)^{2}} d \lambda=\sum_{s=0}^{t+j+l}\left(\begin{array}{c}
t+j+l \\
s
\end{array}\right)\left(-\zeta_{c}\right)^{t+j+l-s} \mathrm{e}^{\zeta_{c}} \int_{\zeta_{c}}^{\infty} v^{s-2} \mathrm{e}^{-v} d v
$$


Therefore, we can write (A.8) as

$$
\begin{aligned}
\mathrm{D}_{c}^{(t)}= & \mathbb{E}\left[\sum_{n=1}^{n_{t}} \frac{\left(\lambda_{n}\right)^{t}}{\left(\lambda_{n}+\zeta_{c}\right)^{2}}\right] \\
= & \sum_{i=1}^{n_{t}} \sum_{j=0}^{i-1} \sum_{l=0}^{i-1}(-1)^{j+l}\left(\begin{array}{c}
i-1 \\
i-1-j
\end{array}\right)\left(\begin{array}{c}
i-1 \\
i-1-l
\end{array}\right) \frac{1}{j ! l !} \\
& \sum_{s=0}^{t+j+l}\left(\begin{array}{c}
t+j+l \\
s
\end{array}\right)\left(-\zeta_{c}\right)^{t+j+l-s} \mathrm{e}^{\zeta_{c}} \int_{\zeta_{c}}^{\infty} v^{s-2} \mathrm{e}^{-v} d v .
\end{aligned}
$$

The integral can be written as

$$
\int_{\zeta_{c}}^{\infty} v^{s-2} \mathrm{e}^{-v} d v= \begin{cases}-\operatorname{Ei}\left(1, \zeta_{c}\right)+\frac{\mathrm{e}^{-\zeta_{c}}}{\zeta_{c}^{2}} & \text { for } s=0 \\ \operatorname{Ei}\left(1, \zeta_{c}\right) & \text { for } s=1 \\ \Gamma\left(s-1, \zeta_{c}\right) & \text { for } s \geq 2\end{cases}
$$

$\operatorname{Ei}(\cdot, \cdot)$ and $\Gamma(\cdot, \cdot)$ are generalized exponential integral and incomplete gamma function, respectively. 


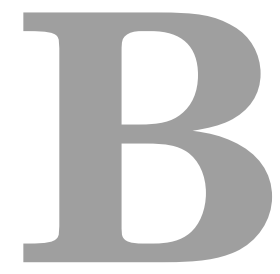

\section{Proof of Result 2}

In this appendix, we prove that when the entries of an $n_{t} \times n_{t}$ matrix $\mathbf{H}$ are i.i.d. $\mathcal{C N}(0,1)$, then the expected value of $\left(\sum_{n=1}^{n_{t}} \frac{\lambda_{n}}{\lambda_{n}+\zeta_{c}}\right)^{2}$, where $\lambda_{n}$ is the $n^{\text {th }}$ eigenvalue of $\mathbf{H H}^{H}$ (uncorrelated central Wishart matrix), with respect to $\lambda_{n}$ $\forall n$, is given by

$$
\begin{aligned}
\mathrm{F}_{c}= & \mathbb{E}\left[\left(\sum_{n=1}^{n_{t}} \frac{\lambda_{n}}{\lambda_{n}+\zeta_{c}}\right)^{2}\right]=\mathrm{D}_{c}^{(2)}+\sum_{i=1}^{n_{t}} \sum_{j=1, j \neq i}^{n_{t}} \\
& \left(\sum_{r=0}^{i-1} \sum_{s=0}^{i-1}(-1)^{r+s}\left(\begin{array}{c}
i-1 \\
i-1-r
\end{array}\right)\left(\begin{array}{c}
i-1 \\
i-1-s
\end{array}\right) \frac{1}{r ! s !} \sum_{b=0}^{1+r+s}\left(\begin{array}{c}
1+r+s \\
b
\end{array}\right)\left(-\zeta_{c}\right)^{1+r+s-b}\right. \\
& \left.\mathrm{e}^{\zeta_{c}} \int_{\zeta_{c}}^{\infty} v^{b-1} \mathrm{e}^{-v} d v\right)^{2}-\left(\sum_{r=0}^{i-1} \sum_{s=0}^{j-1}(-1)^{r+s}\left(\begin{array}{c}
i-1 \\
i-1-r
\end{array}\right)\left(\begin{array}{c}
j-1 \\
j-1-s
\end{array}\right) \frac{1}{r ! s !}\right. \\
& \left.\sum_{b=0}^{1+r+s}\left(\begin{array}{c}
1+r+s \\
b
\end{array}\right)\left(-\zeta_{c}\right)^{1+r+s-b} \mathrm{e}^{\zeta_{c}} \int_{\zeta_{c}}^{\infty} v^{b-1} \mathrm{e}^{-v} d v\right)^{2} .
\end{aligned}
$$


Let, $\mathrm{F}_{c}$ be defined by

$$
\begin{aligned}
\mathrm{F}_{c} & =\mathbb{E}\left[\left(\sum_{n=1}^{n_{t}} \frac{\lambda_{n}}{\lambda_{n}+\zeta_{c}}\right)^{2}\right] \\
& =\mathbb{E}\left[\sum_{n=1}^{n_{t}}\left(\frac{\lambda_{n}}{\lambda_{n}+\zeta_{c}}\right)^{2}\right]+\mathbb{E}\left[\sum_{a=1}^{n_{t}} \sum_{b=1, b \neq a}^{n_{t}}\left(\frac{\lambda_{a}}{\lambda_{a}+\zeta_{c}}\right)\left(\frac{\lambda_{b}}{\lambda_{b}+\zeta_{c}}\right)\right] \\
& =n_{t} \mathbb{E}\left[\sum_{n=1}^{n_{t}}\left(\frac{\lambda_{n}}{\lambda_{n}+\zeta_{c}}\right)^{2}\right]+ \\
& n_{t}\left(n_{t}-1\right) \mathbb{E}\left[\frac{1}{n_{t}\left(n_{t}-1\right)} \sum_{a=1}^{n_{t}} \sum_{b=1, b \neq a}^{n_{t}}\left(\frac{\lambda_{a}}{\lambda_{a}+\zeta_{c}}\right)\left(\frac{\lambda_{b}}{\lambda_{b}+\zeta_{c}}\right)\right],
\end{aligned}
$$

where $\lambda_{a}$ and $\lambda_{b}$ are two distinct arbitrary eigenvalues. Using Result 1 given in Appendix A to solve the first term in (B.2) and denoting $f_{0}\left(\lambda_{a}, \lambda_{b}\right)$ as the joint PDF of two distinct arbitrary eigenvalues, we can write (B.2) as

$$
\mathrm{F}_{c}=\mathrm{D}_{c}^{(2)}+n_{t}\left(n_{t}-1\right) \int_{0}^{\infty} \int_{0}^{\infty}\left(\frac{\lambda_{a}}{\lambda_{a}+\zeta_{c}}\right)\left(\frac{\lambda_{a}}{\lambda_{a}+\zeta_{c}}\right) f_{0}\left(\lambda_{a}, \lambda_{b}\right) d \lambda_{a} d \lambda_{b}
$$

The joint PDF of two distinct arbitrary eigenvalues (for the case $C K=n_{t}$ ) is given by $[3,175]$

$$
f_{0}\left(\lambda_{a}, \lambda_{b}\right)=\frac{1}{n_{t}\left(n_{t}-1\right)} \sum_{i=1}^{n_{t}} \sum_{j=1, j \neq i}^{n_{t}} \mathrm{e}^{-\left(\lambda_{a}+\lambda_{b}\right)} \mathrm{Z}\left(\lambda_{a}, \lambda_{b}\right)
$$

where

$$
\mathrm{Z}\left(\lambda_{a}, \lambda_{b}\right)=\mathrm{L}_{i-1}\left(\lambda_{a}\right)^{2} \mathrm{~L}_{j-1}\left(\lambda_{b}\right)^{2}-\mathrm{L}_{i-1}\left(\lambda_{a}\right) \mathrm{L}_{j-1}\left(\lambda_{a}\right) \mathrm{L}_{i-1}\left(\lambda_{b}\right) \mathrm{L}_{j-1}\left(\lambda_{b}\right)
$$

Now we can write (B.3) as

$$
\mathrm{F}_{c}=\mathrm{D}_{c}^{(2)}+\sum_{i=1}^{n_{t}} \sum_{j=1, j \neq i}^{n_{t}} \underbrace{\int_{0}^{\infty} \int_{0}^{\infty} \frac{\lambda_{a}}{\lambda_{a}+\zeta_{c}} \frac{\lambda_{a}}{\lambda_{a}+\zeta_{c}} \mathrm{e}^{-\left(\lambda_{a}+\lambda_{b}\right)} \mathrm{Z}\left(\lambda_{a}, \lambda_{b}\right) d \lambda_{a} d \lambda_{b}}_{\mathrm{Y}} .
$$

Solving for $\mathrm{Y}$ in (B.6), we get

$$
\begin{aligned}
\mathrm{Y} & =\int_{0}^{\infty} \int_{0}^{\infty}\left(\frac{\mathrm{e}^{-\lambda_{a}} \lambda_{a}}{\lambda_{a}+\zeta_{c}}\right)\left(\frac{\mathrm{e}^{-\lambda_{b}} \lambda_{b}}{\lambda_{b}+\zeta_{c}}\right)\left(\mathrm{L}_{i-1}\left(\lambda_{a}\right)^{2} \mathrm{~L}_{j-1}\left(\lambda_{b}\right)^{2}\right) d \lambda_{a} d \lambda_{b} \\
& -\int_{0}^{\infty} \int_{0}^{\infty}\left(\frac{\mathrm{e}^{-\lambda_{a}} \lambda_{a}}{\lambda_{a}+\zeta_{c}}\right)\left(\frac{\mathrm{e}^{-\lambda_{b}} \lambda_{b}}{\lambda_{b}+\zeta_{c}}\right)\left(\mathrm{L}_{i-1}\left(\lambda_{a}\right) \mathrm{L}_{j-1}\left(\lambda_{a}\right) \mathrm{L}_{i-1}\left(\lambda_{b}\right) \mathrm{L}_{j-1}\left(\lambda_{b}\right)\right) d \lambda_{a} d \lambda_{b}
\end{aligned}
$$


As the double integrals in (B.7) are of the same function but with different variables, we can also write $Y$ by squaring the result of the single integral as

$$
\begin{aligned}
\mathrm{Y} & =\left(\int_{0}^{\infty} \frac{\mathrm{e}^{-\lambda} \lambda}{\lambda+\zeta_{c}} \mathrm{~L}_{i-1}(\lambda)^{2} d \lambda\right)^{2}-\left(\int_{0}^{\infty} \frac{\mathrm{e}^{-\lambda} \lambda}{\lambda+\zeta_{c}} \mathrm{~L}_{i-1}(\lambda) \mathrm{L}_{j-1}(\lambda) d \lambda\right)^{2} \\
& =\left(\int_{0}^{\infty} \frac{\mathrm{e}^{-\lambda} \lambda}{\lambda+\zeta_{c}}\left[\sum_{r=0}^{i-1}(-1)^{r}\left(\begin{array}{c}
i-1 \\
i-1-r
\end{array}\right) \frac{\lambda^{r}}{r !}\right]^{2} d \lambda\right)^{2} \\
& -\left(\int_{0}^{\infty} \frac{\mathrm{e}^{-\lambda} \lambda}{\lambda+\zeta_{c}} \sum_{r=0}^{i-1}(-1)^{r}\left(\begin{array}{c}
i-1 \\
i-1-r
\end{array}\right) \frac{\lambda^{r}}{r !} \sum_{s=0}^{i-1}(-1)^{s}\left(\begin{array}{c}
i-1 \\
i-1-s
\end{array}\right) \frac{\lambda^{s}}{s !} d \lambda\right)^{2} \\
& =\left(\sum_{r=0}^{i-1} \sum_{s=0}^{i-1}(-1)^{r+s}\left(\begin{array}{c}
i-1 \\
i-1-r
\end{array}\right)\left(\begin{array}{c}
i-1 \\
i-1-s
\end{array}\right) \frac{1}{r ! s !} \int_{0}^{\infty} \frac{\lambda^{1+r+s} \mathrm{e}^{-\lambda}}{\left(\lambda+\zeta_{c}\right)} d \lambda\right)^{2} \\
& -\left(\sum_{r=0}^{i-1} \sum_{s=0}^{j-1}(-1)^{r+s}\left(\begin{array}{c}
i-1 \\
i-1-r
\end{array}\right)\left(\begin{array}{c}
j-1 \\
j-1-s
\end{array}\right) \frac{1}{r ! s !} \int_{0}^{\infty} \frac{\lambda^{1+r+s} \mathrm{e}^{-\lambda}}{\left(\lambda+\zeta_{c}\right)} d \lambda\right)^{2} .
\end{aligned}
$$

Solving the integrals in (B.8) by substituting $v=\lambda+\zeta_{c}$ and then substituting the resulting expression in (B.6), we can write (B.6) as

$$
\begin{aligned}
\mathrm{F}_{c}= & \mathbb{E}\left[\left(\sum_{n=1}^{n_{t}} \frac{\lambda_{n}}{\lambda_{n}+\zeta_{c}}\right)^{2}\right]=\mathrm{D}_{c}^{(2)}+\sum_{i=1}^{n_{t}} \sum_{j=1, j \neq i}^{n_{t}} \\
& \left(\sum_{r=0}^{i-1} \sum_{s=0}^{i-1}(-1)^{r+s}\left(\begin{array}{c}
i-1 \\
i-1-r
\end{array}\right)\left(\begin{array}{c}
i-1 \\
i-1-s
\end{array}\right) \frac{1}{r ! s !}\right. \\
& \left.\sum_{b=0}^{1+r+s}\left(\begin{array}{c}
1+r+s \\
b
\end{array}\right)\left(-\zeta_{c}\right)^{1+r+s-b} \mathrm{e}^{\zeta_{c}} \int_{\zeta_{c}}^{\infty} v^{b-1} \mathrm{e}^{-v} d v\right)^{2} \\
& -\left(\sum_{r=0}^{i-1} \sum_{s=0}^{j-1}(-1)^{r+s}\left(\begin{array}{c}
i-1 \\
i-1-r
\end{array}\right)\left(\begin{array}{c}
j-1 \\
j-1-s
\end{array}\right) \frac{1}{r ! s !}\right. \\
& \left.\sum_{b=0}^{1+r+s}\left(\begin{array}{c}
1+r+s \\
b
\end{array}\right)\left(-\zeta_{c}\right)^{1+r+s-b} \mathrm{e}^{\zeta_{c}} \int_{\zeta_{c}}^{\infty} v^{b-1} \mathrm{e}^{-v} d v\right)^{2} .
\end{aligned}
$$

The integral can also be written as

$$
\int_{\zeta_{c}}^{\infty} v^{b-1} \mathrm{e}^{-v} d v= \begin{cases}\operatorname{Ei}\left(1, \zeta_{c}\right) & \text { for } b=0 \\ \Gamma\left(b, \zeta_{c}\right) & \text { for } b \geq 1\end{cases}
$$




\section{Bibliography}

[1] B. Clerckx and C. Oestges, MIMO Wireless Networks: Channels, Techniques and Standards for Multi-Antenna, Multi-User and Multi-Cell Systems. Academic Press, 2013.

[2] J. H. Winters, "On the capacity of radio communication systems with diversity in a Rayleigh fading environment," IEEE J. Sel. Areas Commun., pp. $871-878,1987$.

[3] E. Telatar, "Capacity of multi-antenna Gaussian channels," European Trans. Telecommun., pp. 585 - 595, 1999.

[4] S. Alamouti, "A simple transmit diversity technique for wireless communications," IEEE J. Sel. Areas Commun., vol. 16, no. 8, pp. 1451 - 1458, 1998.

[5] G. J. Foschini and M. J. Gans, "On limits of wireless communications in a fading environment when using multiple antennas," Wireless Pers. Commun., pp. 311 - 335, 1998.

[6] G. J. Foschini, "Layered space-time architecture for wireless communication in a fading environment when using multi-element antennas," Bell Labs Tech. J., pp. $41-59,1996$.

[7] P. W. Wolniansky, G. J. Foschini, G. Golden, and R. Valenzuela, "VBLAST: An architecture for realizing very high data rates over the richscattering wireless channel," in Proc. URSI Int. Symp. Signals, Syst., and Elec., pp. 295 - 300, IEEE, 1998.

[8] T. Lo, "Maximum ratio transmission," in Proc. IEEE Int. Conf. Commun., pp. $1310-1314,1999$.

[9] S. Sanayei and A. Nosratinia, "Antenna selection in MIMO systems," IEEE Commun. Mag., pp. 68 - 73, 2004.

[10] A. Paulraj, R. Nabar, and D. Gore, Introduction to Space-time Wireless Communications. Cambridge University Press, 2003. 
[11] V. Tarokh, N. Seshadri, and A. R. Calderbank, "Space-time codes for high data rate wireless communication: Performance criterion and code construction," IEEE Trans. Inf. Theory, pp. $744-765,1998$.

[12] H. El Gamal, G. Caire, and M. O. Damen, "Lattice coding and decoding achieve the optimal diversity-multiplexing tradeoff of MIMO channels," IEEE Trans. Inf. Theory, pp. 968 - 985, 2004.

[13] L. Zheng and D. N. C. Tse, "Diversity and multiplexing: A fundamental tradeoff in multiple-antenna channels," IEEE Trans. Inf. Theory, pp. 1073 - 1096, 2003.

[14] R. W. Heath Jr. and A. Paulraj, "Switching between diversity and multiplexing in MIMO systems," IEEE Trans. Commun., pp. 962 - 968, 2005.

[15] A. Lozano and N. Jindal, "Transmit diversity vs. spatial multiplexing in modern MIMO systems," IEEE Trans. Wireless Commun., pp. 186 - 197, 2010 .

[16] A. Grant, "Performance analysis of transmit beamforming," IEEE Trans. Commun., pp. 738 - 744, 2005.

[17] A. Narula, M. Lopez, M. Trott, and G. W. Wornell, "Efficient use of side information in multiple-antenna data transmission over fading channels," IEEE J. Sel. Areas Commun., pp. 1423 - 1436, 1998.

[18] G. Caire and S. Shamai, "On the achievable throughput of a multiantenna Gaussian broadcast channel," IEEE Trans. Inf. Theory, pp. $1691-1706$, 2003.

[19] H. Viswanathan, S. Venkatesan, and H. Huang, "Downlink capacity evaluation of cellular networks with known-interference cancellation," IEEE J. Sel. Areas Commun., pp. 802 - 811, 2003.

[20] D. J. Love, R. W. Heath Jr., V. K. N. Lau, D. Gesbert, B. D. Rao, and M. Andrews, "An overview of limited feedback in wireless communication systems," IEEE J. Sel. Areas Commun., pp. 1341 - 1365, 2008.

[21] R. W. Heath Jr. and A. Paulraj, "A simple scheme for transmit diversity using partial channel feedback," in Proc. IEEE Asilomar Conf. on Signals, Systems, and Comp., pp. 1073 - 1078, 1998.

[22] C. K. Au-Yeung and D. J. Love, "On the performance of random vector quantization limited feedback beamforming in a MISO system," IEEE Trans. Wireless Commun., pp. 458 - 462, 2007. 
[23] A. Goldsmith, Wireless Communications. Cambridge University Press, 2005 .

[24] D. Tse and P. Viswanath, Fundamentals of Wireless Communication. Cambridge University Press, 2005.

[25] T. Yoo and A. Goldsmith, "Optimality of zero-forcing beamforming with multiuser diversity," in Proc. IEEE Int. Conf. Commun., vol. 1, pp. 542 - 546, IEEE, 2005.

[26] D. Gesbert, M. Kountouris, R. W. Heath Jr., C.-B. Chae, and T. Salzer, "Shifting the MIMO paradigm," IEEE Signal Process. Mag., pp. 36 - 46, 2007.

[27] N. Jindal, "MIMO broadcast channels with finite-rate feedback," IEEE Trans. Inf. Theory, pp. 5045 - 5060, 2006.

[28] T. Yoo and A. Goldsmith, "On the optimality of multiantenna broadcast scheduling using zero-forcing beamforming," Selected Areas in Communications, IEEE Journal on, pp. 528 - 541, 2006.

[29] S. Shamai and B. M. Zaidel, "Enhancing the cellular downlink capacity via co-processing at the transmitting end," in Proc. IEEE Veh. Technol. Conf., pp. 1745 - 1749, 2001.

[30] H. Zhang and H. Dai, "Cochannel interference mitigation and cooperative processing in downlink multicell multiuser MIMO networks," EURASIP Journal on Wireless Communications and Networking, pp. 222 - 235, 2004 .

[31] D. Gesbert, S. Hanly, H. Huang, S. Shamai Shitz, O. Simeone, and W. Yu, "Multi-cell MIMO cooperative networks: A new look at interference," IEEE J. Sel. Areas Commun., pp. 1380 - 1408, 2010.

[32] F. Rusek, D. Persson, B. K. Lau, E. G. Larsson, T. L. Marzetta, O. Edfors, and F. Tufvesson, "Scaling up MIMO: Opportunities and challenges with very large arrays," IEEE Sig. Process. Mag., pp. 40 - 60, 2013.

[33] E. Larsson, O. Edfors, F. Tufvesson, and T. Marzetta, "Massive MIMO for next generation wireless systems," IEEE Commun. Mag., pp. 186 $195,2014$.

[34] T. L. Marzetta, "Noncooperative cellular wireless with unlimited numbers of base station antennas," IEEE Trans. Wireless Commun., pp. 3590 3600, 2010. 
[35] J. Jose, A. Ashikhmin, T. Marzetta, and S. Vishwanath, "Pilot contamination and precoding in multi-cell TDD systems," IEEE Trans. Wireless Commun., pp. 2640 - 2651, 2011.

[36] B. Hochwald, T. Marzetta, and V. Tarokh, "Multiple-antenna channel hardening and its implications for rate feedback and scheduling," IEEE Trans. Inf. Theory, pp. 1893 - 1909, 2004.

[37] C. Neil, M. Shafi, P. Smith, and P. Dmochowski, "On the impact of antenna topologies for massive MIMO systems," Proc. IEEE Int. Conf. on Commun. (ICC), 2015.

[38] F. Kaltenberger, H. Jiang, M. Guillaud, and R. Knopp, "Relative channel reciprocity calibration in MIMO/TDD systems," in Future Network and Mobile Summit, pp. 1 - 10, IEEE, 2010.

[39] J.-C. Guey and L. D. Larsson, "Modeling and evaluation of MIMO systems exploiting channel reciprocity in TDD mode," in Proc. IEEE Veh. Technol. Conf., pp. 4265 - 4269, IEEE, 2004.

[40] R. Habendorf and G. Fettweis, "Pre-equalization for TDD systems with imperfect transceiver calibration," in Proc. IEEE Veh. Technol. Conf., pp. 1369 - 1373, IEEE, 2008.

[41] Q. Li, G. Li, W. Lee, M. il Lee, D. Mazzarese, B. Clerckx, and Z. Li, "MIMO techniques in WiMAX and LTE: A feature overview," IEEE Commun. Mag., no. 5, pp. $86-92,2010$.

[42] D. Love, R. W. Heath Jr., and T. Strohmer, "Grassmannian beamforming for multiple-input multiple-output wireless systems," IEEE Trans. Inf. Theory, pp. 2735 - 2747, 2003.

[43] 3GPP TS 36.211, "Evolved universal terrestrial radio access (E-UTRA), physical channels and modulation," vol. V10.0.0, 2011.

[44] D. J. Love and R. W. Heath Jr., "Limited feedback diversity techniques for correlated channels," IEEE Trans. Veh. Tech., pp. 718 - 722, 2006.

[45] V. Raghavan, R. W. Heath Jr., and A. M. Sayeed, "Systematic codebook designs for quantized beamforming in correlated MIMO channels," IEEE J. Sel. Areas Commun., pp. 1298 - 1310, 2007.

[46] J. Choi, B. Clerckx, N. Lee, and G. Kim, "A new design of polar-cap differential codebook for temporally/spatially correlated MISO channels," IEEE Trans. Wireless Commun., pp. 703 - 711, 2012. 
[47] C. K. Au-Yeung, D. J. Love, and S. Sanayei, "Trellis coded line packing: Large dimensional beamforming vector quantization and feedback transmission," IEEE Trans. Wireless Commun., pp. 1844 - 1853, 2011.

[48] J. Choi, Z. Chance, D. Love, and U. Madhow, "Noncoherent trellis coded quantization: A practical limited feedback technique for massive MIMO systems," IEEE Trans. Commun., pp. 5016 - 5029, 2013.

[49] R. Bhagavatula and R. W. Heath Jr., "Adaptive limited feedback for sumrate maximizing beamforming in cooperative multicell systems," IEEE Trans. Signal Process., pp. 800 - 811, 2011.

[50] J. Zhang and J. G. Andrews, "Adaptive spatial intercell interference cancellation in multicell wireless networks," IEEE J. Sel. Areas Commun., pp. $1455-1468,2010$.

[51] S. Yu, H.-B. Kong, Y.-T. Kim, S.-H. Park, and I. Lee, "Novel feedback bit allocation methods for multi-cell joint processing systems," IEEE Trans. Wireless Commun., pp. 3030 - 3036, 2012.

[52] F. Yuan, C. Yang, G. Wang, and M. Lei, "Adaptive channel feedback for coordinated beamforming in heterogeneous networks," IEEE Trans. Wireless Communications, pp. 3980 - 3994, 2013.

[53] C.-N. Chuah, J. M. Kahn, and D. Tse, "Capacity of multi-antenna array systems in indoor wireless environment," in Proc. IEEE Glob. Telecom. Conf., pp. 1894 - 1899, 1998.

[54] D.-S. Shiu, G. J. Foschini, M. J. Gans, and J. M. Kahn, "Fading correlation and its effect on the capacity of multielement antenna systems," IEEE Trans. Commun., pp. $502-513,2000$.

[55] S. Loyka, "Channel capacity of MIMO architecture using the exponential correlation matrix," IEEE Commun. Lett., pp. 369 - 371, 2001.

[56] T. S. Rappaport, Wireless communications: principles and practice, vol. 2. Prentice Hall, 1996.

[57] ITU-R M.2135, "Guidelines for evaluation of radio interface technologies for imt-advanced," Report, 2009.

[58] 3GPP TR 25.996 V.6, "Spatial channel model for multiple input multiple output (MIMO) simulations (Release 6)," Technical Report, 2003.

[59] IST-WINNER Deliverable D5.4 v.1.4, "Final report on link level and system level channel models," IST-WINNER, Technical Report, 2005. 
[60] IST-WINNER II Deliverable 1.1.2 v.1.2, "WINNER II channel models," IST-WINNER2, Technical Report, 2007.

[61] WINNER + Deliverable D5.3 v.1.0, "D5.3: WINNER + Final Channel Models," WINNER+, Technical Report, 2010.

[62] L. Hentilä, P. Kyösti, M. Käske, M. Narandzic, and M. Alatossava, "Matlab implementation of the WINNER Phase II channel model ver1.1 [online]," IST-WINNER-II Project, 2007.

[63] C. Jiang, M. Wang, F. Shu, J. Wang, W. Sheng, and Q. Chen, "Multi-user MIMO with limited feedback using alternating codebooks," IEEE Trans. Commun., pp. 333 - 338, 2012.

[64] D. Sacristan-Murga and A. Pascual-Iserte, "Differential feedback of MIMO channel Gram matrices based on geodesic curves," IEEE Trans. Wireless Commun., pp. 3714 - 3727, 2010.

[65] J. Chang, I.-T. Lu, and Y. Li, "Adaptive codebook based channel prediction and interpolation for multiuser MIMO-OFDM systems," in Proc. IEEE Int. Conf. on Commun. (ICC), pp. 1 - 5, 2011.

[66] Y. Zhang, D. Zhu, Z. Sun, and M. Lei, "Predictive unitary precoding for spatial multiplexing systems in temporally correlated MIMO channels with delayed limited feedback," in Proc. IEEE Global Telecommun. Conf. (GLOBECOM), pp. $1-6,2011$.

[67] L. Godara, "Application of antenna arrays to mobile communications. ii. beam-forming and direction-of-arrival considerations," IEEE Proceedings, pp. 1195 - 1245, 1997.

[68] M. Zhang, M. Shafi, P. J. Smith, and P. A. Dmochowski, "Precoding performance with codebook feedback in a MIMO-OFDM system," in Proc. IEEE Int. Conf. Commun. (ICC), p. 1 - 6, 2011.

[69] J. Mirza, P. Dmochowski, P. J. Smith, and M. Shafi, "Capacity loss for multilayer codebook precoding in MIMO systems," in Proc. IEEE Int. Symp. Personal Indoor and Mobile Radio Commun. (PIMRC), p. $1890-$ $1895,2012$.

[70] W. Santipach and M. Honig, "Asymptotic capacity of beamforming with limited feedback," in Proc. Int. Symp. Inf. Theory, p. 290, 2004.

[71] D. Ryan, I. Vaughan, L. Clarkson, I. Collings, D. Guo, and M. Honig, "QAM codebooks for low-complexity limited feedback MIMO beamforming," in Proc. IEEE Int. Conf. on Commun. (ICC), pp. 4162 - 4167, 2007. 
[72] D. Love and R. W. Heath Jr., "Equal gain transmission in multiple-input multiple-output wireless systems," IEEE Trans. Commun., pp. 1102 1110, 2003.

[73] W. Santipach and M. Honig, "Capacity of beamforming with limited training and feedback," in Proc. IEEE Int. Symp. on Inf. Theory, (ISIT), pp. $376-380,2006$.

[74] D. Love and R. W. Heath Jr., "Limited feedback unitary precoding for spatial multiplexing systems," IEEE Trans. Inf. Theory, pp. 2967 - 2976, 2005 .

[75] D. Love and R. W. Heath Jr., "Multimode precoding for MIMO wireless systems," IEEE Trans. Signal Process., pp. 3674 - 3687, 2005.

[76] P. Baier, M. Meurer, T. Weber, and H. Troger, "Joint transmission (JT), an alternative rationale for the downlink of time division CDMA using multi-element transmit antennas," in Proc. IEEE Int. Symp. Spread Spectrum Techniques and Applications, pp. 1 - 5, 2000.

[77] C. Peel, B. Hochwald, and A. Swindlehurst, "A vector-perturbation technique for near-capacity multiantenna multiuser communication-part I: Channel inversion and regularization," IEEE Trans. Commun., pp. 195 $-202,2005$.

[78] M. Sadek, A. Tarighat, and A. H. Sayed, "A leakage-based precoding scheme for downlink multi-user MIMO channels," IEEE Trans. Wireless Commun., pp. 1711 - 1721, 2007.

[79] J. H. Conway, R. H. Hardin, and N. J. A. Sloane, "Packing lines, planes, etc.: Packings in Grassmannian spaces," 1996.

[80] W. Santipach and K. Mamat, "Optimal feedback interval for temporallycorrelated multiantenna channel," in Proc. IEEE Global Telecommun. Conf. (GLOBECOM), pp. 1 - 5, 2011.

[81] T. Kim, D. Love, and B. Clerckx, "A feedback update control scheme for limited feedback multiple antennas systems," in Proc. IEEE Global Telecommun. Conf. (GLOBECOM), pp. 1 - 5, 2010.

[82] T. Kim, D. Love, and B. Clerckx, "Leveraging temporal correlation for limited feedback multiple antennas systems," in Proc. IEEE Int. Conf. Acoustics Speech and Signal Process., pp. 3422 - 3425, 2010.

[83] T. Kim, D. Love, and B. Clerckx, "MIMO systems with limited rate differential feedback in slowly varying channels," IEEE Trans. Commun., pp. 1175-1189, 2011. 
[84] M. Zhang, P. J. Smith, M. Shafi, P. A. Dmochowski, and J. Mirza, "MIMO capacity gain analysis for general channel models," in Proc. IEEE Int. Conf. Commun., pp. 4846 - 4851, 2012.

[85] X. Song and H.-N. Lee, "Multimode precoding for MIMO systems: Performance bounds and limited feedback codebook design," IEEE Trans. Signal Process., pp. 5296 - 5301, 2008.

[86] J. Zheng and B. D. Rao, "Capacity analysis of MIMO systems using limited feedback transmit precoding schemes," IEEE Trans. Signal Process., pp. 2886 - 2901, 2008.

[87] J. C. Roh and B. D. Rao, "Design and analysis of MIMO spatial multiplexing systems with quantized feedback," IEEE Trans. Signal Process., pp. $2874-2886,2006$.

[88] A. Dabbagh and D. Love, "Feedback rate-capacity loss tradeoff for limited feedback MIMO systems," IEEE Trans. Inf. Theory, pp. 2190 - 2202, 2006.

[89] D. J. Love and R. W. Heath Jr., "Limited feedback precoding for spatial multiplexing systems using linear receivers," in Proc. IEEE Military Commun. Conf., pp. 627 - 632, 2003.

[90] N. Jindal, "Antenna combining for the MIMO downlink channel," IEEE Trans. Wireless Commun., pp. 3834 - 3844, 2008.

[91] N. Ravindran and N. Jindal, "Limited feedback-based block diagonalization for the MIMO broadcast channel," IEEE J. Sel. Areas in Commun., pp. $1473-1482,2008$.

[92] H. Chae, Y. Kim, J. Yang, B.-C. Ihm, and D. K. Kim, "Multimode random beamforming for multiuser downlink MIMO system with limited feedback," in Proc. IEEE Veh. Tech. Conf., pp. $837-841,2008$.

[93] B. Clerckx, G. Kim, and S. Kim, "Correlated fading in broadcast MIMO channels: Curse or blessing?," in Proc. IEEE Glob. Telecom. Conf., pp. 1 $-5,2008$.

[94] V. Raghavan and V. Veeravalli, "On quantized multi-user beamforming in spatially correlated broadcast channels," in Proc. IEEE Int. Symp. Inf. Theory, pp. 2041 - 2045, 2007.

[95] C. Wang and R. Murch, "Adaptive downlink multi-user MIMO wireless systems for correlated channels with imperfect CSI," IEEE Trans. Wireless Commun., pp. 2435 - 2446, 2006. 
[96] M. Trivellato, F. Boccardi, and H. Huang, "On transceiver design and channel quantization for downlink multiuser MIMO systems with limited feedback," IEEE J. Sel. Areas in Commun., pp. 1494 - 1504, 2008.

[97] B. Clerckx, G. Kim, and S. Kim, "MU-MIMO with channel statisticsbased codebooks in spatially correlated channels," in Proc. IEEE Glob. Telecom. Conf., pp. 1 - 5, 2008.

[98] T. Inoue and R. W. Heath Jr., "Grassmannian predictive coding for limited feedback multiuser MIMO systems," in Proc. IEEE Int. Conf. Acoustics, Speech and Signal Process., pp. 3076 - 3079, 2011.

[99] T. Inoue and R. W. Heath Jr., "Grassmannian predictive coding for delayed limited feedback MIMO systems," in Proc. Annual Allerton Conf. Commun., Control, and Comput.,, pp. 783 - 788, 2009.

[100] T. Inoue and R. W. Heath Jr., "Geodesic prediction for limited feedback multiuser MIMO systems in temporally correlated channels," in Proc. IEEE Radio and Wireless Symp., pp. 167 - 170, 2009.

[101] T. Inoue and R. W. Heath Jr., "Grassmannian predictive frequency domain compression for limited feedback beamforming," in Proc. Inf. Theory and App. Workshop, pp. 1 - 5, 2010.

[102] K. Kim, I. H. Kim, and D. Love, "Utilizing temporal correlation in multiuser MIMO feedback," in Proc. Asilomar Conf. Signals, Systems and Computers, pp. $121-125,2008$.

[103] T. Kim, D. Love, and B. Clerckx, "MIMO systems with limited rate differential feedback in slowly varying channels," IEEE Trans. Commun., pp. $1175-1189,2011$.

[104] J. Choi, B. Clerckx, and D. Love, "Differential codebook for general rotated dual-polarized MISO channels," in Proc. IEEE Glob. Telecom. Conf., pp. $4222-4227,2012$.

[105] IEEE C802.16m-08/1187, "Evaluation of CL SU and MU MIMO codebooks," 2008.

[106] IEEE C802.16m-09/0279r2, "Proposed text of MIMO sections for the IEEE 802.16m amendment," 2009.

[107] IEEE C802.16m-08/1182r1, "Codebook design for IEEE 802.16m MIMO schemes," 2008.

[108] IEEE C802.16m-09/0058r3, "Differential feedback for IEEE 802.16m MIMO schemes," 2009. 
[109] A. Firag and P. Smith, "Adaptive codebook implementation for a simple MRT-MRC system," Technical Report, 2011.

[110] J. Mirza, P. Dmochowski, P. Smith, and M. Shafi, "Limited feedback multiuser MISO systems with differential codebooks in correlated channels," in Proc. IEEE Int. Conf. Commun., pp. 3979 - 3984, 2013.

[111] J. Mirza, P. Dmochowski, P. Smith, and M. Shafi, "A differential codebook with adaptive scaling for limited feedback MU MISO systems," IEEE Wireless Commun. Lett., pp. 2 - 5, 2014.

[112] J. Mirza, P. Smith, M. Shafi, P. Dmochowski, A. Firag, and A. Papathanassiou, "Double-cap differential codebook structure for MU MISO systems in correlated channels," IEEE Wireless Commun. Lett., pp. 441 $-444,2014$.

[113] L. Zheng and D. Tse, "Communication on the Grassmann manifold: a geometric approach to the noncoherent multiple-antenna channel," IEEE Trans. Inf. Theory, pp. 359 - 383, 2002.

[114] T. Kim, D. J. Love, B. Clerckx, and S. Kim, "Differential rotation feedback MIMO system for temporally correlated channels," in Proc. IEEE Glob. Telecom. Conf., pp. 1 - 5, IEEE, 2008.

[115] J.-C. Guey and L. Larsson, "Modeling and evaluation of MIMO systems exploiting channel reciprocity in tdd mode," in Proc. IEEE Veh. Tech. Conf. (VTC), pp. $4265-4269,2004$.

[116] J. Choi, D. J. Love, and P. Bidigare, "Downlink training techniques for FDD massive MIMO systems: Open-loop and closed-loop training with memory," IEEE J. Sel. Topics Sig. Process., pp. 802 - 814, 2014.

[117] P.-H. Kuo, H. Kung, and P.-A. Ting, "Compressive sensing based channel feedback protocols for spatially-correlated massive antenna arrays," in Proc. IEEE Wireless Commun.and Networking Conf., pp. 492 - 497, 2012.

[118] J. Mirza, M. Shafi, P. Smith, and P. Dmochowski, "Limited feedback massive MISO systems with trellis coded quantization for correlated channels," submitted to IEEE Trans. Veh. Tech., 2015.

[119] L. Yu, W. Liu, and R. Langley, "SINR analysis of the subtraction-based SMI beamformer," IEEE Trans. Signal Process., pp. 5926 - 5932, 2010.

[120] J. Choi, D. Love, and T. Kim, "Trellis-extended codebooks and successive phase adjustment: A path from LTE-Advanced to FDD massive MIMO systems," IEEE Trans. Wireless Commun., pp. 1-1, 2014. 
[121] W. M. Newman and R. F. Sproull, Principles of Interactive Computer Graphics. McGraw-Hill, 1979.

[122] N. L. Johnson, S. Kotz, and N. Balakrishnan, Continuous Univariate Distributions, vol. 1. John Wiley \& Sons, 1994.

[123] J.-S. Jiang and M. Ingram, "Distributed source model for short-range MIMO," in Proc. IEEE Veh. Tech. Conf. (VTC), pp. 357 - 362, 2003.

[124] A. A. Abouda, H. M. El-Sallabi, and S. Häggman, "Effect of antenna array geometry and ULA azimuthal orientation on MIMO channel properties in urban city street grid," Progress In Electromagnetics Research, vol. 64, pp. $257-278,2006$.

[125] X. Gao, O. Edfors, F. Rusek, and F. Tufvesson, "Massive MIMO performance evaluation based on measured propagation data," IEEE Trans. Wireless Commun., [Online]. Available: http://arxiv.org/abs/1403.3376, submitted for publication.

[126] S. Shamai and B. M. Zaidel, "Multi-cell communications: An information theoretic perspective," in Proc. Joint Workshop on Commun. and Coding, pp. $14-17,2004$.

[127] J. Zhang, R. Chen, J. Andrews, and R. W. Heath Jr., "Coordinated multicell MIMO systems with cellular block diagonalization," in Proc. Asilomar Conf. on Signal, Syst. and Comput., pp. 1669 - 1673, 2007.

[128] N. Lee and W. Shin, "Adaptive feedback scheme on K-cell MISO interfering broadcast channel with limited feedback," IEEE Trans. on Wireless Commun., pp. $401-406,2011$.

[129] M. Karakayali, G. J. Foschini, R. Valenzuela, and R. D. Yates, "On the maximum common rate achievable in a coordinated network," in Proc. IEEE Int. Conf. Commun., pp. 4333 - 4338, 2006.

[130] M. K. Karakayali, G. J. Foschini, and R. A. Valenzuela, "Network coordination for spectrally efficient communications in cellular systems," IEEE Wireless Commun., pp. 56 - 61, 2006.

[131] A. D. Wyner, "Shannon-theoretic approach to a Gaussian cellular multipleaccess channel," IEEE Trans. Info. Theory, pp. 1713 - 1727, 1994.

[132] L. Venturino, N. Prasad, and X. Wang, "Coordinated linear beamforming in downlink multi-cell wireless networks," IEEE Trans. Wireless Commun., pp. 1451 - 1461, 2010. 
[133] J. Zhang, C.-K. Wen, S. Jin, X. Gao, and K.-K. Wong, "Large system analysis of cooperative multi-cell downlink transmission via regularized channel inversion with imperfect CSIT," IEEE Trans. Wireless Commun., pp. $4801-4813,2013$.

[134] R. Muharar, R. Zakhour, and J. Evans, "Base station cooperation with feedback optimization: A large system analysis," IEEE Trans. Inf. Theory, pp. $3620-3644,2014$.

[135] J. Mirza, P. Smith, and M. Dmochowski, P.A.and Shafi, "Coordinated regularized zero-forcing precoding for multicell MISO systems with limited feedback," under prep., 2015.

[136] B. Ozbek and D. Le Ruyet, "Adaptive limited feedback for intercell interference cancelation in cooperative downlink multicell networks," in Proc. Int. Symp. Wireless Commun. Systems (ISWCS), 2010.

[137] S. W. Kim and A. Goldsmith, "Truncated power control in code-division multiple-access communications," IEEE Trans. on Veh. Tech., pp. 965 $972,2000$.

[138] L. Thiele, M. Schellmann, S. Schiffermuller, V. Jungnickel, and W. Zirwas, "Multi-cell channel estimation using virtual pilots," in Proc. IEEE Veh. Tech. Conf., (VTC), pp. $1211-1215,2008$.

[139] R. Muharar, R. Zakhour, and J. Evans, "Optimal power allocation and user loading for multiuser MISO channels with regularized channel inversion," IEEE Trans. Commun., pp. 5030 - 5041, 2013.

[140] A. Dabbagh and D. Love, "Multiple antenna MMSE based downlink precoding with quantized feedback or channel mismatch," IEEE Trans. Commun., pp. 1859 - 1868, 2008.

[141] T. Yoo and A. Goldsmith, "Capacity and power allocation for fading MIMO channels with channel estimation error," IEEE Trans. Info. Theory, pp. $2203-2214,2006$.

[142] E. Park, H. Kim, H. Park, and I. Lee, "Feedback bit allocation schemes for multi-user distributed antenna systems," IEEE Commun. Lett., pp. 99 $-102,2013$.

[143] G. Liu, X. Su, G. Wu, and S. Li, "Coordinated scheduling with adaptive feedback in cooperative multi-cell downlink system," in IEEE Int. Conf. Commun. China (ICCC), pp. 647 - 652, 2012. 
[144] A. Kammoun, A. Muller, E. Bjornson, and M. Debbah, "Linear precoding based on polynomial expansion: Large-scale multi-cell MIMO systems," IEEE J. Sel. Topics Sig. Process., pp. 861 - 875, 2014.

[145] D. H. Nguyen and T. Le-Ngoc, "MMSE precoding for multiuser MISO downlink transmission with non-homogeneous user SNR conditions," EURASIP J. Advances in Signal Process., 2014.

[146] 3GPP TR 36.814, "Further advancements for E-UTRA physical layer aspects," V9.0.0, 2010.

[147] K. Huang, R. W. Heath Jr., and J. Andrews, "Limited feedback beamforming over temporally-correlated channels," IEEE Trans. Signal Process., pp. 1959 - 1975, 2009.

[148] Y. Isukapalli and B. Rao, "Finite rate feedback for spatially and temporally correlated MISO channels in the presence of estimation errors and feedback delay," in Proc. IEEE Glob. Telecom. Conf., pp. 2791 - 2795, 2007.

[149] S. H. Ting, K. Sakaguchi, and K. Araki, "A markov-kronecker model for analysis of closed-loop MIMO systems," IEEE Commun. Lett., vol. 10, no. 8, pp. $617-619,2006$.

[150] E. Au, S. Jin, M. McKay, W. H. Mow, X. Gao, and I. Collings, "Analytical performance of MIMO-SVD systems in ricean fading channels with channel estimation error and feedback delay," IEEE Trans. Wireless Commun., vol. 7, no. 4, pp. $1315-1325,2008$.

[151] H. T. Nguyen, J. Andersen, and G. Pedersen, "Capacity and performance of MIMO systems under the impact of feedback delay," in Proc. IEEE Int. Sym. Personal Indoor and Mobile Radio Commun., vol. 1, pp. 53 - 57, 2004.

[152] K. Kobayashi, T. Ohtsuki, and T. Kaneko, "MIMO systems in the presence of feedback delay," in Proc. IEEE Int. Conf. Commun. (ICC), vol. 9, pp. $4102-4106,2006$.

[153] A. Duel-Hallen, "Fading channel prediction for mobile radio adaptive transmission systems," IEEE Proceedings, vol. 95, no. 12, pp. 2299 - 2313, 2007.

[154] J. Choi and R. Heath, "Interpolation based transmit beamforming for MIMO-OFDM with limited feedback," vol. 53, no. 11, pp. 4125 - 4135, 2005 . 
[155] T. Pande, D. Love, and J. Krogmeier, "Reduced feedback MIMO-OFDM precoding and antenna selection," IEEE Trans. Signal Process., vol. 55, no. 5, pp. $2284-2293,2007$.

[156] C. Kosta, B. Hunt, A. Quddus, and R. Tafazolli, "On interference avoidance through inter-cell interference coordination (ICIC) based on OFDMA mobile systems," IEEE Commun. Surveys Tuts., pp. 973 - 995, 2013.

[157] S. Akoum, M. Kountouris, and R. W. Heath Jr., "On imperfect csi for the downlink of a two-tier network," in Proc. IEEE Int. Symp. Inf. Theory (ISIT), pp. $553-557,2011$.

[158] C. Masouros, M. Sellathurai, and T. Ratnarajah, "Selective vector perturbation for low-power small cell MISO downlinks," in IEEE Global Commun. Conf. (GLOBECOM), pp. 3343 - 3348, 2014.

[159] H. Tataria, M. Shafi, P. Smith, and P. Dmochowski, "Coordinated two-tier heterogeneous cellular networks with leakage based beamforming," CoRR, vol.abs/1503.01566, 2014.

[160] Q. Cui, H. Wang, P. Hu, X. Tao, P. Zhang, J. Hamalainen, and L. Xia, "Evolution of limited-feedback CoMP systems from 4G to 5G: CoMP features and limited-feedback approaches," IEEE Veh. Tech. Mag., pp. $94-$ 103, 2014.

[161] O. El Ayach, S. Rajagopal, S. Abu-Surra, Z. Pi, and R. W. Heath Jr., "Spatially sparse precoding in millimeter wave MIMO systems," IEEE Trans. Wireless Commun., pp. 1499 - 1513, 2014.

[162] A. Alkhateeb, O. El Ayach, G. Leus, and R. W. Heath Jr., "Channel estimation and hybrid precoding for millimeter wave cellular systems," IEEE J. Sel. Topics Signal Process., pp. 831 - 846, 2014.

[163] J. Murdock, E. Ben-Dor, Y. Qiao, J. Tamir, and T. Rappaport, "A 38 $\mathrm{GHz}$ cellular outage study for an urban outdoor campus environment," in Proc. IEEE Wireless Commun. and Net. Conf. (WCNC), pp. $3085-$ 3090, 2012.

[164] J. Wang, Z. Lan, C.-W. Pyo, T. Baykas, C.-S. Sum, M. Rahman, J. Gao, R. Funada, F. Kojima, H. Harada, and S. Kato, "Beam codebook based beamforming protocol for multi-Gbps millimeter-wave WPAN systems," IEEE J. Sel. Areas Commun., pp. 1390-1399, 2009.

[165] S. Hur, T. Kim, D. Love, J. Krogmeier, T. Thomas, and A. Ghosh, "Millimeter wave beamforming for wireless backhaul and access in small cell networks," IEEE Trans. Commun., pp. 4391-4403, 2013. 
[166] J. Brady, N. Behdad, and A. Sayeed, "Beamspace MIMO for millimeterwave communications: System architecture, modeling, analysis, and measurements," IEEE Trans. Antennas Propag., pp. 3814 - 3827, 2013.

[167] A. Alkhateeb, O. El Ayach, G. Leus, and R. W. Heath Jr., "Hybrid precoding for millimeter wave cellular systems with partial channel knowledge," in Information Theory and Applications Workshop (ITA), pp. 1-5, 2013.

[168] A. Alkhateeb, G. Leus, and R. W. Heath Jr., "Limited feedback hybrid precoding for multi-user millimeter wave systems," IEEE Trans. Wireless Commun., [Online]. Available: http://arxiv.org/abs/1409.5162, submitted for publication.

[169] R. A. Stirling-Gallacher and Md. Saifur Rahman, "Multi-user MIMO strategies for a millimeter wave communication system using hybrid beamforming," in Proc. IEEE Int. Conf. Commun. (ICC), 2015.

[170] Z. Xu, S. Han, Z. Pan, and I. Chih-Lin, "Alternating beamforming methods for hybrid analog and digital MIMO transmission," in Proc. IEEE Int. Conf. Commun. (ICC), 2015.

[171] O. El Ayach, R. Heath, S. Abu-Surra, S. Rajagopal, and Z. Pi, "The capacity optimality of beam steering in large millimeter wave MIMO systems," in IEEE Int. Workshop Signal Process. Advances Wireless Commun. (SPAWC), pp. 100 - 104, 2012.

[172] D. Ying, F. W. Vook, T. A. Thomas, and D. J. Love, "Hybrid structure in massive MIMO: Achieving large sum rate with fewer RF chains," in Proc. IEEE Int. Conf. Commun. (ICC), 2015.

[173] M. K. Samimi and T. S. Rappaport, "3-D statistical channel model for millimeter-wave outdoor mobile broadband communications," in Proc. IEEE Int. Conf. Commun. (ICC) (to be appear), 2015.

[174] T. Thomas, H. C. Nguyen, G. MacCartney, and T. Rappaport, "3D mmwave channel model proposal," in Proc. IEEE Veh. Tech. Conf., pp. 1 $-6,2014$.

[175] P. J. Smith and M. Shafi, "On a Gaussian approximation to the capacity of wireless MIMO systems," in Proc. IEEE Int. Conf. on Commun. (ICC), pp. $406-410,2002$. 